\title{
PNNL-13845
}

\section{orfict or}

\section{BOAFDING}

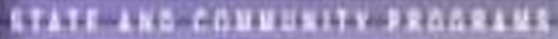

\section{Documentation for}

FY2003 BTS GPRA Metrics

Building Technology, State and Community Programs

Energy Efficiency and Renewable Energy

U.S. Department of Energy

April 2002

Prepared for Building Technology,

State and Community Programs

by Pacific Northwest National Laboratory 


\title{
DISCLAIMER
}

This report was prepared as an account of work sponsored by an agency of the United States Government. Neither the United States Government nor any agency thereof, nor Battelle Memorial Institute, nor any of their employees, makes any warranty, express or implied, or assumes any legal liability or responsibility for the accuracy, completeness, or usefulness of any information, apparatus, product, or process disclosed, or represents that its use would not infringe privately owned rights. Reference herein to any specific commercial product, process, or service by trade name, trademark, manufacturer, or otherwise does not necessarily constitute or imply its endorsement, recommendation, or favoring by the United States Government or any agency thereof, or Battelle Memorial Institute. The views and opinions of authors expressed herein do not necessarily state or reflect those of the United States Government or any agency thereof.

\author{
PACIFIC NORTHWEST NATIONAL LABORATORY \\ operated by \\ BATTELLE \\ for the \\ UNITED STATES DEPARTMENT OF ENERGY \\ under Contract DE-AC06-76RL01830
}

This document was printed on recycled paper. 


\section{Documentation for}

\section{FY2003 BTS GPRA Metrics}

Building Technology, State and Community Programs

Energy Efficiency and Renewable Energy

U.S. Department of Energy

D. B. Belzer

K. A. Cort

J. A. Dirks

D. J. Hostick

R. H. Pool

April 2002

Prepared for Building Technology, State and Community Programs the U.S. Department of Energy under Contract DE-AC06-76RL01830

Pacific Northwest National Laboratory

Richland, Washington 99352 


\section{Executive Summary}

Pacific Northwest National Laboratory (PNNL) estimated the fiscal year (FY) 2003 energy, environmental, and financial benefits (i.e., metrics) of the technologies and practices in the U.S. Department of Energy's (DOE's) Office of Building Technology, State and Community Programs (BTS). BTS is part of DOE's Office of Energy Efficiency and Renewable Energy (EE), which uses the estimates of benefits as part of its annual budget request.

This effort is referred to as GPRA Metrics because the Government Performance and Results Act (GPRA) of 1993 mandates such estimates of benefits, which are submitted to EE's Office of Planning, Budget, and Management as part of EE's budget request.

This report includes a series of sections that detail the approach and methodology used to estimate future energy, environmental, and financial benefits produced by technologies and practices supported by BTS in the FY 2003. An overview is describes the GPRA process and the models used to estimate savings. Forecasted benefits for all programs through 2030 are included in tables. The results of the forecasted energy savings, consumer cost savings, and carbon benefits for each of the 18 BTS programs are included in individual program summaries, and overall results of the FY 2003 GPRA efforts are summarized for all BTS programs. Technical appendixes include the FY 2003 GPRA data call and descriptions of the models used, baseline assumptions, and diffusion curve estimates. 
Overview 


\section{Overview of the FY 2003 GPRA Metrics Process}

Pacific Northwest National Laboratory (PNNL) estimated the FY 2003 energy, environmental, and financial benefits (i.e., metrics) of the technologies and practices in the U.S. Department of Energy's (DOE's) Office of Building Technology, State and Community Programs (BTS). BTS falls with DOE's Office of Energy Efficiency and Renewable Energy $(E E)$, which uses the estimates of benefits as part of its annual budget request.

The metrics effort was initiated by EE in 1994 to develop quantitative measures of program benefits and costs. The Government Performance and Results Act (GPRA) of 1993 mandates such estimates of benefits, which are submitted to EE's Office of Planning, Budget, and Management (OPBM) as part of EE's budget request. The supporting analysis and data are used to set strategic goals and objectives within BTS and DOE, to communicate the benefits of EE programs to all interested parties, and to defend the budget before OPMB and Congress.

\section{Estimating the Energy Savings of BTS Programs}

Energy savings for the FY 2003 GPRA metrics were based on the FY 2003 budget request and estimated at a program level and then aggregated to the decision unit level. Benefits were estimated for 37 BTS programs or technologies and then rolled up into 17 program groups and then into 7 BTS decision units, as shown in Table 1. BTS's 7 decision units fall into one of two broad areas:

- Building Research and Standards, which develops, implements, and coordinates research and development $(R \& D)$ that improves the energy efficiency of building components and then uses system design and regulatory activities to integrate these components into building energy systems. ${ }^{1}$

- Building Technology Assistance, which is responsible for accelerating the adoption of energy efficiency and renewable building technologies through technical and financial assistance to states and local communities. ${ }^{2}$

Several different approaches are required to estimate the benefits of the wide array of BTS programs. This section briefly describes the analytical approaches used to estimate energy savings for BTS's FY 2003 budget request. Greater detail on each BTS program is provided later in this document in program-specific summaries.

The benefits of EE programs and technologies were assessed at an aggregated level as decision units (formerly known as planning units) to simplify cross-sector comparisons and to limit the number of elements being evaluated to a manageable number. Likewise, the benefits were assessed for a limited number of defined metrics:

\footnotetext{
1 “BTS Building Research and Standards Mission Statement," FY 2003 Budget Request (internal BTS document).

2 “BTS Office of Building Technology Assistance Mission Statement," FY 2003 Budget (internal BTS document).
} 
Table 1. Decision Units and Programs Evaluated for the FY 2003 GPRA Metrics

\begin{tabular}{|c|c|c|}
\hline Decision Units & BTS Programs or Technologies & $\begin{array}{c}\text { BTS Programs Aggregated for } \\
\text { GPRA FY } 2003 \text { Metrics }\end{array}$ \\
\hline State Energy & - State Formula Grants & - State Formula Grants \\
\hline $\begin{array}{l}\text { Weatherization } \\
\text { Assistance }\end{array}$ & - Weatherization Assistance Program & $\begin{array}{l}\text { - Weatherization Assistance } \\
\text { Program }\end{array}$ \\
\hline $\begin{array}{l}\text { Community Energy } \\
\text { Program }\end{array}$ & $\begin{array}{l}\text { - Rebuild America } \\
\text { - Information Outreach } \\
\text { - Training and Assistance for Codes }\end{array}$ & $\begin{array}{l}\text { - Rebuild America } \\
\text { - Information Outreach } \\
\text { - Training and Assistance for } \\
\text { Codes }\end{array}$ \\
\hline Energy Star Program & $\begin{array}{l}\text { - Energy Star: Clothes Washers } \\
\text { - Energy Star: Refrigerators } \\
\text { - Energy Star: Electric Water Heaters } \\
\text { - Energy Star: Gas Water Heaters } \\
\text { - Energy Star: Room Air Conditioner } \\
\text { - Energy Star: Compact Fluorescent Lights } \\
\text { - Energy Star: Dishwashers }\end{array}$ & - Energy Star \\
\hline $\begin{array}{l}\text { Residential Buildings } \\
\text { Integration } \\
\end{array}$ & $\begin{array}{l}\text { - Residential Technology R\&D } \\
\text { - Residential Building Codes }\end{array}$ & $\begin{array}{l}\text { - Residential Technology R\&D } \\
\text { - Residential Building Codes }\end{array}$ \\
\hline $\begin{array}{l}\text { Commercial Buildings } \\
\text { Integration }\end{array}$ & $\begin{array}{l}\text { - Commercial Technology R\&D } \\
\text { - Commercial Building Codes }\end{array}$ & $\begin{array}{l}\text { - Commercial Technology R\&D } \\
\text { - Commercial Building Codes }\end{array}$ \\
\hline $\begin{array}{l}\text { Equipment, Materials, } \\
\text { and Tools }\end{array}$ & $\begin{array}{l}\text { - Lighting R\&D: Two-Photon Phosphors } \\
\text { - Lighting R\&D: Solid State Lighting } \\
\text { - Lighting R\&D: Controls } \\
\text { - Refrigeration \& Thermal Distribution R\&D: Residential } \\
\text { HVAC Distribution System } \\
\text { - Refrigeration \& Thermal Distribution R\&D: Advanced } \\
\text { Electric Heat Pump Water Heater } \\
\text { - Refrigeration \& Thermal Distribution R\&D: Refrigerant } \\
\text { - Reter } \\
\text { - Refrigeration \& Thermal Distribution R\&D: Commercial } \\
\text { - Emergeration } \\
\text { - Emerging Technologies R\&D: Heat Pump Water Heater } \\
\text { - Emerging Technologies R\&D: Roof Top Air Conditioning } \\
\text { - Eeater } \\
\text { - Emerging Tech R\&D: Recessed Can Lights } \\
\text { - Building Envelope R\&D: Electrochromic Windows } \\
\text { - Building Envelope R\&D: Superwindows } \\
\text { - Building Envelope R\&D: Quick-Fill Walls } \\
\text { - Building Envelope R\&D: R30 Insulation/30 Year Life Roofs } \\
\text { - Building Envelope R\&D: Moisture/Wet Insulation } \\
\text { - Design Strategies and Assistance } \\
\text { - Lighting and Appliance Standards: Residential Gas } \\
\text { Furnaces/Boilers } \\
\text { - Lighting and Appliance Standards: EPAct Standards } \\
\text { - Lighting and Appliance Standards: Distribution } \\
\text { Transformers }\end{array}$ & $\begin{array}{l}\text { - Lighting R\&D } \\
\text { - Refrigeration and Thermal } \\
\text { Distribution R\&D } \\
\text { - Emerging Technologies R\&D } \\
\text { - Building Envelope R\&D: } \\
\text { Windows } \\
\text { - Building Envelope R\&D: } \\
\text { Thermal Insulation and } \\
\text { Building Materials } \\
\text { - Design Strategies and } \\
\text { Assistance } \\
\text { - Lighting and Appliance } \\
\text { Standards }\end{array}$ \\
\hline
\end{tabular}


- energy savings

- environmental benefits

- economic/financial metrics.

Environmental and economic benefits (energy cost savings) were keyed directly to energy savings. Therefore, the balance of this overview focuses on just the energy-savings' estimates.

For most BTS programs, estimates were broken out by building sector, building type, region, vintage, end use, fuel type, and type of equipment displaced and then aggregated to obtain the program or technology benefits. The program and decision unit structure used reflected the structure used in the FY 2003 budget request. The analysis considered program goals, technology characteristics (including performance and cost), the targeted market, and program milestones. The technologies and practices modeled were chosen as representing a specific program. Not all activities funded by BTS are modeled; activities were selected if they met some minimal threshold of funding and are likely to result in measurable energy savings.

The program characteristics were developed through extensive interaction with the BTS Office Directors and Program Managers. For FY 2003, program characterization summaries were based on information gathered during interviews conducted in August 2001. The program characterizations were then reviewed and revised during meetings with BTS Program Managers. The program characterizations presented in subsequent sections of this document represent the results of those interviews.

\section{Analysis Changes for FY 2003 GPRA}

Before EE released the FY 2003 GPRA data call, the National Research Council issued a report entitled Energy Research at DOE: Was It Worth It? The report assessed the outcomes of energy efficiency and fossil energy research from 1978 to 2000 . One of the report's recommendations to consider for assessing research development and deployment programs was that "DOE should adopt an analytic framework similar to that used by this committee as a uniform methodology for assessing the benefits and costs of its R\&D programs. DOE should also use this type of analytic framework of this sort in reporting to Congress under GPRA."

The National Research Council report assumed that the private sector would have developed the technology in the absence of DOE five years after DOE realized the benefits. As part of the GPRA data call, EE asked the sectors to consider what would have happened in the absence of the EE program and to identify benefits relating only to EE's effort.

This change was implemented within the BTS estimates by determining BTS programs that act as acceleration-to-market programs versus those that would not have been developed or implemented in the absence of government funding. Further detail as to how this was implemented at the program level is contained in the "Detailed Results" section of this document, and is referred to as the National Academy of Sciences or "NAS methodology." 


\section{Modeling Methods Used in Estimating Benefits}

The BTS GPRA estimates of benefits were calculated using one of three methods:

- National Energy Modeling System (NEMS)

- Building Energy Savings Estimation Tool (BESET)

- Spreadsheets designed for a specific program.

NEMS can link the costs and benefit characteristics of a technology and its market penetration. However, NEMS has difficulty representing some BTS technologies, such as the whole-building programs because NEMS is designed to model specific technologies and not variable groups of technologies.

BESET was built specifically for estimating the benefits of BTS programs and therefore allows various types of programs to be characterized, including wholebuilding, envelope, and equipment programs. The major disadvantage of BESET is that the penetration rates (i.e., fraction of sales or fraction of installed base) are determined outside the model and therefore are not explicitly linked to the program's cost and benefit characteristics. In addition, BESET cannot model BTS equipment that competes against more than one baseline equipment type.

For programs that are not easily modeled in BESET or NEMS, spreadsheets were used. For example, because BTS's codes and standards programs have already developed its own set of spreadsheet tools for estimating impacts of the building codes programs, these tools were adapted for the GPRA estimation process. Each of the three methods used for deriving energy-saving estimates for the FY 2002 GPRA metrics is described in more detail in the following subsections.

\section{NEMS}

Many of BTS's technology programs were modeled using NEMS. The commercial and residential energy demand modules within NEMS were used to calculate the savings generated by the improved BTS technologies. Energy savings in equipment programs were calculated by comparing new equipment efficiencies with baseline efficiencies. ${ }^{3}$

The NEMS commercial and residential demand modules generate forecasts of energy demand (energy consumption) for those sectors. The commercial demand module generates fuel consumption forecasts for electricity, natural gas, and distillate fuel oil. These forecasts are based on energy prices and macroeconomic variables from the NEMS system, combined with external data sources. The residential model uses energy prices and macroeconomic indicators to generate energy consumption by fuel type and census division in the residential sector.

NEMS selects specific technologies to meet the energy services demands by choosing among a discrete set of technologies that are exogenously characterized by commercial availability,

${ }^{3}$ For theFY 2003 metrics, theNE MS model associated with the Energy I nformation Administration's Annual Energy Outl ook 2000 was used.

Overview - 4 


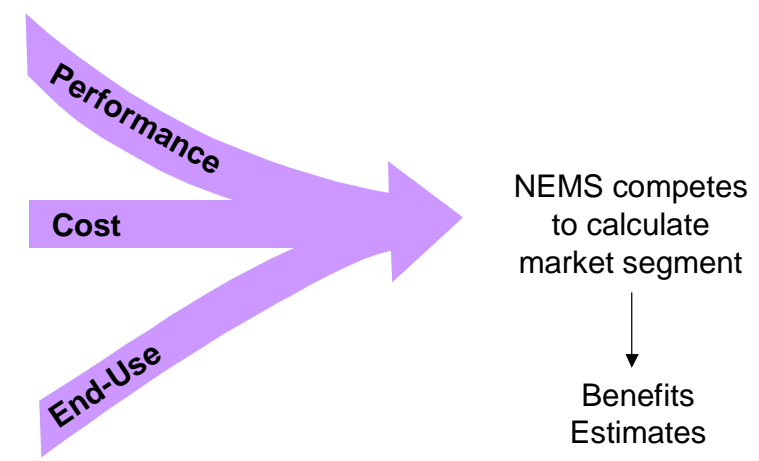

Figure 1. Developing the Market Segment (NEMS)

capital costs, operating and maintenance costs, efficiencies, and lifetime (see Figure 1). NEMS is coded to allow several possible assumptions to be used about consumer behavior to model this selection process. For the GPRA effort, the menu of equipment was changed to include relevant BTS program equipment, technological innovations, and standards.

The NEMS design can accommodate various technology choices. For the GPRA FY 2003 metrics, the NEMS data input was adjusted to reflect BTS technology choices. For BTS programs that target shell efficiency, specific shell-efficiency indices were read into the model.

\section{BESET}

BESET is a bottom-up accounting model that compares baseline energy use against the BTS technology. BESET also is used to centrally collect, store, and report all results produced by all the various estimation methods. Finally, BESET produces the input files needed for estimating employment impacts developed in a separate modeling environment.

BESET can estimate benefits for various programs: whole building, envelope, lighting, HVAC, cogeneration, and water heating. BESET also contains a "tax" al gorithm that calculates the average energy savings per budget dollar for the BTS portfolio so the energy savings can be applied to an umbrella program. Beginning with the FY 2001 GPRA effort, BESET was primarily used to model BTS programs that target whole-building energy use. Although BESET can model equipment and envelope programs, NEMS estimates those programs.

To determine energy savings for specific BTS programs, BESET requires information in the following areas:

- Program Performance Goals. The goals of each program are assessed in terms of energy savings (e.g., percent load reductions and equipment efficiency improvements) and used as inputs to BESET.

- Target Market. Target markets are defined in terms of building sector (e.g. residential and commercial), building type (e.g. single family and commercial education), size (commercial only), income level (residential only), vintage (e.g., new or existing), and climate zone or region. Using the Rebuild America program as an example, Figure 2 illustrates the process used to define the program's targeted market segment within BESET. 


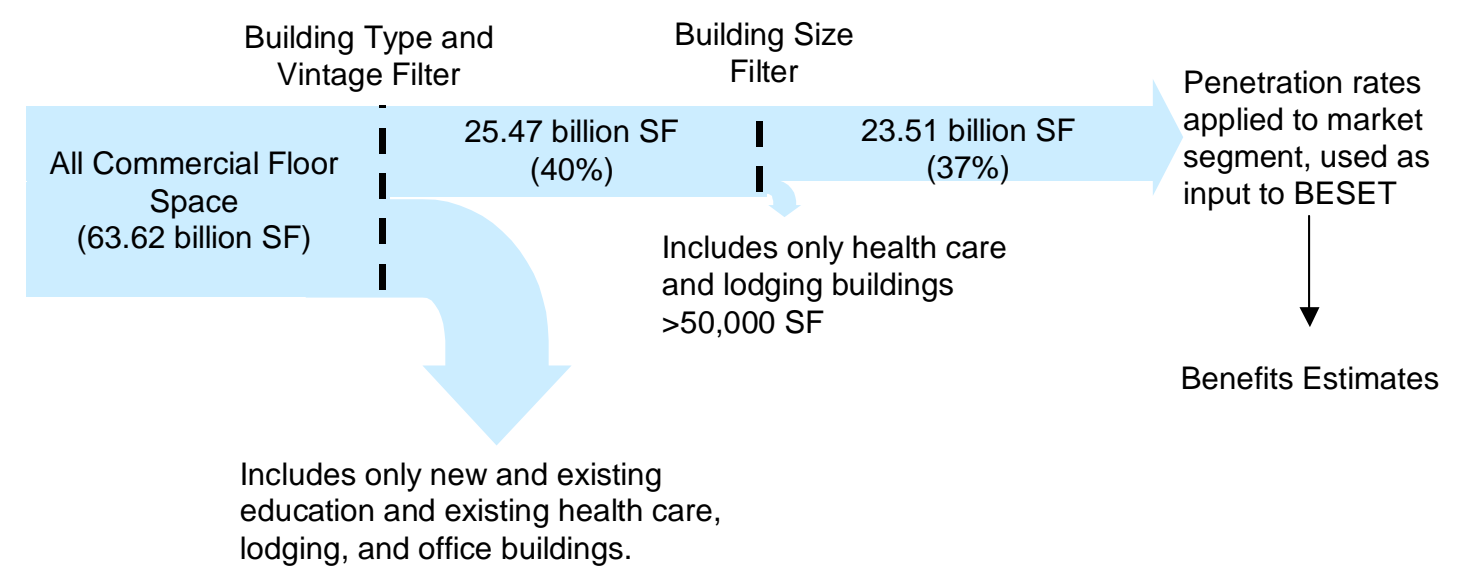

Figure 2. Developing the Market Segment (NEMS): Rebuild America Example

Once the target market has been identified, the penetration into that market is determined using technology diffusion curves (discussed later in this section). Within BESET, market penetration is defined as either the fraction of sales for equipment for new buildings or the fraction of installed base for existing buildings. The penetration model requires only the year of introduction into the market, an estimate of market penetration in 2020 (provided by BTS Program Managers), and the selection of the most appropriate diffusion curve category.

- Private Investment (Cost). Estimates of private investment for both the baseline and the BTS technology or practice are entered into BESET. I deally, the investment costs would be considered when market penetration is developed; however, the current diffusion model used does not incorporate costs at this time. In addition to private investment, non-energy savings program benefits are also quantified when possible and entered into BESET.

The basic steps involved in calculating the energy savings for whole-building programs modeled in BESET are as follows:

1. Determine the size of the potential market.

2. Determine the number of units affected by the BTS program.

3. Determine the base space conditioning, water heating and other end-use loads if appropriate.

4. Determine the space conditioning and water heating end-use loads after the program is implemented.

5. Calculate the energy savings.

All estimates were aggregated through a BESET-NEMS interface. BESET contains a report generator that aggregates the program and technology level benefits into the decision units. The aggregated information is submitted to OPBM to include in the GPRA metrics effort for all EE sectors. 


\section{Spreadsheet Models}

Whenever possible, programs were modeled within NEMS or BESET to help ensure consistency in baseline inputs and methodology. However, several BTS programs were modeled in spreadsheets because of their unique characteristics. The estimated savings generated by the spreadsheet models were entered by fuel type into "fixed" tables within BESET so that the environmental and energy cost-savings' benefits can be calculated using the same data set as the other programs. Spreadsheets were used to model the following programs:

- State Formula Grants. This program was modeled based on historical information that provides an estimated level of savings per program dollar. Because neither BESET nor NEMS are designed for this type of analysis, the program continued to be modeled in a separate spreadsheet.

- Weatherization Assistance Program. This program was modeled based on program studies that provide per-household savings' estimates. While these inputs may be able to be translated into load reductions and the program run through BESET, such an effort has not been undertaken. The primary barrier to incorporating this program into BESET is that fuel mix for houses in the target market is significantly different between the BESET baseline and historical Weatherization program data.

- Information Outreach. The estimates for the FY 2003 request and appropriation were adopted directly from a study commissioned by BTS (Messersmith and Azimi 2000).

- Building Codes. Building code activities are spread among three BTS decision units. However, because of the interrelationships between the three, savings were estimated for the building codes and standards as a whole. Savings estimates were then allocated among the three primary funding sources:

- Training and Assistance for Codes (within the Community Energy Program decision unit)

- Residential Building Energy Codes (within Residential Buildings Integration decision unit)

- Commercial Building Energy Codes (within Commercial Buildings Integration decision unit).

The long-term impact of DOE's assistance to code activities is based largely on data de veloped for internal use in building codes and standards. DOE provides a high level of support for states seeking to adopt new energy codes, either based on ASHRAE Standard 90.1 or the International Energy Conservation Code (IECC) (previously the Model Energy Code). Several states have self-developed codes that are not supported by building codes and standards and are not counted in the estimates of program impact.

- Refrigeration and Thermal Distribution R\&D. The refrigeration savings' estimates were based on a report on end-use consumption produced by PNNL, program goals, and other various data sources (Belzer and Wrench 1997). Energy-savings estimates were developed in a spreadsheet model because commercial refrigeration is a service, not a specific piece of equipment, and therefore cannot be modeled in NEMS or BESET. 
- Lighting and Appliance Standards: EPAct Standards and Distribution Transformers. For FY 2003, the energy savings from Energy Policy Act of 1992 (EPAct) standards were based on a spreadsheet developed by PNNL specifically to support an EPAct screening analysis conducted in late 1999 and early 2000. Because distribution transformers are part of the electricity distribution system, not the building system, transformers cannot be modeled in either NEMS or BESET. Saving estimates were based on a study by Geller and Nadel (1992).

\section{Baseline Inputs}

To the extent possible, the underlying assumptions about building stock forecasts, equipment efficiencies, market shares, and end-use loads were consistent across tools (i.e., NEMS, BESET, and spreadsheets). This consistency was accomplished by drawing most of the baseline characterization data from the Energy Information Administration (EIA), a statistical agency within DOE. For example, the same version of NEMS used in this document was used to produce EIA's Annual Energy Outlook.

For programs modeled in NEMS, consistency is ensured not only across these programs but also with EIA forecasts. BESET also has a baseline characterization, which is drawn from NEMS, EIA's Annual Energy Outlook, the "Residential Energy Consumption Survey," and the "Commercial Buildings Energy Consumption Survey." The consistency of the baseline assumptions of the spreadsheet tools is verified against EIA's data.

\section{Budget Adjustment Process}

The program characterizations that are key to the benefits' estimates were developed through close interaction with the BTS Program Managers. The characterizations require the Program Manager to make assumptions based on the requested level of funding and then describe what would be accomplished at that level. However, the budget request amount sometimes changes between the time the characterization is developed and the time the benefits estimates are required. Changes also occur between the final budget request (on which the final estimates are based) and the actual allocation (for which benefits estimates have also been developed to assist in planning).

For small changes in budget levels, a basic "budget adjustment" is made to the program estimates. It is assumed that to get to $X$ savings, a total of $Y$ budget must be spent, where $Y$ is the cumulative budget. A change in the annual budget results in a change in the cumulative budget. Revised savings are calculated for each year as old savings in year z (new cumulative budget in year z/old cumulative budget in year z). This adjustment mechanism implicitly suggests that either the fraction of expected sales or the performance of the program has changed but does not explicitly tie the change to one factor or the other.

For larger changes, the program inputs are revisited with the BTS Program Managers to determine the impact of a reduced (or increased) budget. Options include changing the year of market introduction, changing the impact on sales (market penetration), modifying the performance objective, and adding or removing tasks or technologies within the program (e.g., 
increased funding in Energy Star may result in developing an Energy Star rating for an additional technology).

\section{Technology Diffusion Curves}

In 1998, a study was conducted by David Belzer, PNNL, to examine the historical market penetration (i.e., diffusion) for 10 energy-efficient products related to the building sector. Diffusion models were estimated for each product based on the specification proposed by Frank Bass in the late 1960s. The resulting models were incorporated into the GPRA metrics analysis for many of the programs and technologies not modeled within the NEMS framework. The model development and empirical analysis were designed to generate more credible predictions of the adoption process of important energy-efficiency technologies in the buildings sector.

The technologies were placed into four separate categories: lighting, HVAC and refrigeration (HVAC/R), envelope, and design. Two additional categories were added: 1) "Other Equipment" represents an average of lighting and HVAC/refrigeration technologies and 2) "Other Program" represents the envelope category. See Appendix C for a summary of this study.

\section{Contents of this Document}

The remainder of this report consists of 18 program descriptions, summarizing information about program's objective, long-term goals, and market and its savings in terms of primary energy savings, carbon equivalent reductions, and consumer cost savings.

Four appendixes provide more detailed information on topics covered in this document. Appendix A details the baseline scenario and inputs used for the FY 2003 metrics. Appendix B contains the GPRA Data Call for FY 2003. Appendix C provides more detailed information on the development of the technology diffusion curves. Appendix D provides detail on the GPRA methodology.

\section{References and Bibliography}

Annual Energy Outlook 1995. 1995. Energy Information Administration, Washington, D.C.

Annual Energy Outlook 2000. 1999. Energy I nformation Administration, Washington, D.C.

Annual Energy Outlook 2001. 2000. Energy I nformation Administration, Washington, D.C.

Appliance Magazine. 1998. “Life Expectancy/Replacement Picture,” 55(9):71.

ASHRAE/IES 90.1. "Energy Standard for New Commercial Buildings." American Society of Heating, Refrigeration, and Air-Conditioning Engineers and Illuminating Engineering Society.

ASHRAE Standard 90a-1980, "Energy Efficient Design of New Buildings Except New LowRise Residential Buildings," American Society of Heating, Refrigeration, and Air-Conditioning Engineers. 
ASHRAE Standard 90.1-1989, "Energy Efficient Design of New Buildings Except New Low-Rise Residential Buildings," American Society of Heating, Refrigeration, and AirConditioning Engineers.

ASHRAE Standard 90.1-1999, "Energy Standard for Buildings Except Low-Rise Residential Buildings," American Society of Heating, Refrigeration, and Air-Conditioning Engineers.

Belzer, D.B and L.E. Wrench. 1997. End-Use Consumption Estimates for U.S. Commercial Buildings, 1992. PNNL-11514, Pacific Northwest National Laboratory, Richland, Washington.

Berry, L.G., M.A. Brown, and L.F. Kinney. 1997. Progress Report of the National Weatherization Assistance Program. ORNL/CON-450, Oak Ridge National Laboratory, Oak Ridge, Tennessee.

Brookhaven National Laboratory. 2001. Better Duct Systems for Home Heating and Cooling. BNL-68167, Volumes 3 and 4, Upton, New York.

Brown, M.A., L.G. Bery, R.A. Balzer, and E. Faby. 1993. National Impacts of the Weatherization Assistance Program in SingleFamily and Small Multifamily Dwellings. ORNL/ CON-326, Oak Ridge National Laboratory, Oak Ridge, Tennessee.

BTS Core Data Book (internal BTS document). 1999. http://www.btscoredatabook.net

"BTS Building Research and Standards Mission Statement." FY 2003 Budget Request, U.S. Department of Energy, Office of Building Technology, State and Community Programs, Washington, D.C.

"BTS Office of Building Technology Assistance Mission Statement." FY 2003 Budget Request, U.S. Department of Energy, Office of Building Technology, State and Community Programs, Washington, D.C.

"Building Technology, State and Community Programs Funding Profile for the FY 2003" (internal BTS document).

"Case for a National Research Program on Semiconductor Lighting," Roland Haitz and Fred Kish, Hewlitt-Packard Co.; J eff Tsao and J eff Nelson, Sandia National Laboratories. White paper presented at the 1999 Optoelectronics Industry Development Association forum in Washington D.C., October 6, 1999.

"Commercial Buildings Energy Consumption Survey." Latest Edition. U.S. Department of Energy, Energy Information Administration, Washington, D.C. eia.doc.gov/emeu/cbecs/ contents.html

Corporate Review Budget (internal BTS document).

Energy Policy Act of 1992, Public Law 102-486.

FY 2001 GPRA Program Characterization (internal BTS document).

Overview - 10 
Geller, H. and S. Nadel. 1992. "Consensus National Efficiency Standards for Lamps, Motors, Showerheads and Faucets, and Commercial HVAC Equipment." In 1992 America for an Energy-Efficient Economy Proceedings, pp. 6.71-6.82.

Gordon, K.L. and M.R. Ledbetter. 2001. Technology Procurement Screening Study, Pacific Northwest National Laboratory, Richland, Washington.

Government Performance and Results Act of 1993.

Messersmith, J ., and S.A. Azimi. August 2000. Communication Effectiveness Analysis for GPRA, Technol ogists, Inc.

National Appliance Energy Conservation Act of 1987, Public Law 100-12.

National Research Council. 2001. Energy Research at DOE: Was it Worth it? National Academy Press, Washington, D.C.

Pacific Northwest National Laboratory. 1998. Facility Energy Decision System User's Guide, Release 4.0. PNNL-10542, Rev. 2, Richland, Washington.

"Residential Energy Consumption Survey." Latest Edition. U.S. Department of Energy, Energy Information Administration, Washington, D.C. eia.doc.gov/emeu/recs/ contents.html

U.S. Department of Energy. 2002. "Commercial Buildings Determinations, Explanation of the Analysis and Spreadsheet (90_1savingsanalysis.xls)."

Łtttp://www.energycodes.gov/implement/determinations_com.stm>

U.S. Department of Energy, Office of Building Technology, State and Community Programs (internal documents) FY 2002 Budget Requests:

Data Bucket Report for Analysis Tools and Design Strategies Program

Data Bucket Report for Appliances and Emerging Technology Program

Data Bucket Report for Building Envelope: Thermal Insulation and Building Materials

Program

Data Bucket Report for Building Envelope: Windows Program

Data Bucket Report for Commercial Building Codes Program

Data Bucket Report for Commercial Building Integration R\&D Program

Data Bucket Report for Energy Star Program

Data Bucket Report for Information Outreach Program

Data Bucket Report for Lighting and Appliance Standards Program

Data Bucket Report for Lighting R\&D Program

Data Bucket Report for Rebuild America Program

Data Bucket Report for Residential Building Integration R\&D Program

Data Bucket Report for Space Conditioning and Refrigeration: Refrigeration Program

Data Bucket Report for State Formula Grants Program

Data Bucket Report for Technology Roadmaps and New and Innovative R\&D

Data Bucket Report for Weatherization Assistance Program

Data Bucket Report for Residential Building Codes Program 


\section{List of Terms}

BESET Building Energy Savings Estimation Tool

BTS

COP

Office of Building Technology, State and Community Programs

DOE

EE

EER

EIA

EPACt

EREC

GPRA

IEEC

NEMS

OPBM

ORNL

PNNL

$R \& D$

SEER

SEP

coefficient of performance

U.S. Department of Energy

DOE's Office of Energy Efficiency and Renewable Energy

energy efficiency ratio

Energy Information Administration

Energy Policy Act of 1992

Energy Efficiency and Renewable Energy Clearinghouse

Government Performance and Results Act of 1993

International Energy Conservation Code

National Energy Modeling System

Office of Planning, Budget, and Management

Oak Ridge National Laboratory

Pacific Northwest National Laboratory

research and development

seasonal energy efficiency ratio

State Energy Program 


\section{Summary of Results}




\section{Summary of Results: BTS Primary Energy Savings Forecasts Based on FY 2003 BTS Budget Request}

The results of the forecasted energy savings, consumer cost savings, and carbon benefits for each of the 17 BTS programs (for 2003, 2010, and 2020) are included in the program summaries in this document. The next section in this document contains tables with forecasted benefits up to the year 2030 for all programs and decision units. The following benefit estimates are included:

- Energy Savings Benefits Tables (TBtu/yr)

- Total Primary Energy Savings

- Primary Electricity savings

- Primary Non-Electric Savings

- Site Electricity Savings

- Site Natural Gas Savings

- Site Oil Savings

- Environmental Benefits Tables (million metric tons per year [MMT/yr])

- Carbon Equivalent Emissions Reductions

- $\mathrm{SO}_{2}$ Emissions Reductions

- $\mathrm{NO}_{x}$ Emissions Reductions

- CO Emissions Reductions

- PM Emissions Reductions

- VOC Emissions Reductions

- Financial Benefits Tables (million \$/yr)

- Consumer Cost Savings

- Non-Energy Cost Savings.

\section{Energy Savings Analysis by Decision Unit}

Decision unit benefits are reported annually. The energy savings' estimates for 2010 represent energy saved in 2010 only. These are not cumulative benefits estimates. However, the energy savings in 2010 are a function of all program activities from FY 2003 on, so the number of affected buildings is a cumulative value. For example, the energy saved in 2010 from the compact fluorescent lights programs is the energy saved in 2010 only from all buildings that have had such lights installed any time between FY 2003 and FY 2010.

Table 1 summarizes the primary energy savings, the carbon equivalent reductions, and the consumer cost savings for the seven BTS decision units. Total primary energy savings for all BTS programs are estimated to reach 0.9 quadrillion Btu (QBtu) by year 2010 and 2.8 QBtu by year 2020. Figure 1 charts annual energy savings for all programs for all years from FY 2003 to 2020. Roughly half of the savings are generated in the residential sector and half in the commercial sector. 
Table 1 Summary of Benefits: Analyses of BTS Programs

\begin{tabular}{|c|c|c|c|c|c|}
\hline Decision Unit & $\begin{array}{c}\text { FY } 2003 \text { Budget } \\
\text { Request (million \$) }\end{array}$ & 2003 & 2005 & 2010 & 2020 \\
\hline \multicolumn{6}{|l|}{ Primary Energy Savings (TBtu/yr) } \\
\hline State Energy Program & 39 & 3.9 & 11.5 & 27.7 & 47.7 \\
\hline Weatherization Assistance Program & 277 & 8.4 & 33.4 & 65.7 & 122.5 \\
\hline Community Energy Program & 20 & 44.1 & 122.4 & 201.5 & 353.0 \\
\hline Energy Star Program & 6 & 15.2 & 41.1 & 169.0 & 568.1 \\
\hline Residential Buildings Integration & 13 & 0.2 & 0.9 & 12.2 & 74.0 \\
\hline Commercial Buildings Integration & 5 & 1.0 & 4.0 & 41.5 & 238.8 \\
\hline Building Equipment and Materials & 30 & 12.4 & 59.3 & 367.5 & 1359.3 \\
\hline Totals & & 85.2 & 264.6 & 885.1 & 2763.3 \\
\hline \multicolumn{6}{|c|}{ Carbon Equivalent Emission Reductions (MMT/yr) } \\
\hline State Energy Program & 39 & 0.1 & 0.2 & 0.5 & 0.9 \\
\hline Weatherization Assistance Program & 277 & 0.1 & 0.4 & 1.1 & 2.0 \\
\hline Community Energy Program & 20 & 0.8 & 2.2 & 3.9 & 6.6 \\
\hline Energy Star Program & 6 & 0.3 & 0.7 & 3.4 & 10.9 \\
\hline Residential Buildings Integration & 13 & 0.0 & 0.0 & 0.2 & 1.2 \\
\hline Commercial Buildings Integration & 5 & 0.0 & 0.1 & 0.8 & 4.4 \\
\hline Building Equipment and Materials & 30 & 0.2 & 1.1 & 7.1 & 24.9 \\
\hline Totals & & 1.5 & 4.7 & 17.0 & 51.0 \\
\hline \multicolumn{6}{|l|}{ Consumer Cost Savings (million $\$ / y r$ ) } \\
\hline State Energy Program & 39 & 24.0 & 70.0 & 177.0 & 345.0 \\
\hline Weatherization Assistance Program & 277 & 59.0 & 175.0 & 469.0 & 917.0 \\
\hline Community Energy Program & 20 & 281.0 & 756.0 & 1344.0 & 2723.0 \\
\hline Energy Star Program & 6 & 107.0 & 288.0 & 1375.0 & 5373.0 \\
\hline Residential Buildings Integration & 13 & 1.0 & 6.0 & 89.0 & 575.0 \\
\hline Commercial Buildings Integration & 5 & 6.0 & 25.0 & 275.0 & 1820.0 \\
\hline Building Equipment and Materials & 30 & 84.0 & 395.0 & 2612.0 & 10792.0 \\
\hline Totals & & 563.0 & 1716.0 & 6342.0 & 22545.0 \\
\hline
\end{tabular}

Figure 2 compares the BTS program primary energy savings projections with EIA's Annual Energy Outlook building energy consumption forecasts. The FY 2003 estimates include only savings for programs that are included in the FY 2003 BTS funding request. Some activities funded in previous years may contribute to total BTS future energy savings but are not in the FY 2003 request. For example, a program that supports a rulemaking that is completed in FY 2002 would not be included in the FY 2003 request; however, this program would produce energy savings in future years.

Figure 2 shows savings for FY 2003 programs as well as for programs that have been retired since FY 2000 but have future energy savings. The BTS program savings projections are charted relative to the building energy consumption forecasts generated by the Annual Energy Outlook 2001. Figure 2 shows that if the forecasted savings generated by BTS programs are subtracted from forecasted total building energy use, total primary building energy use remains relatively flat through 2020 . 


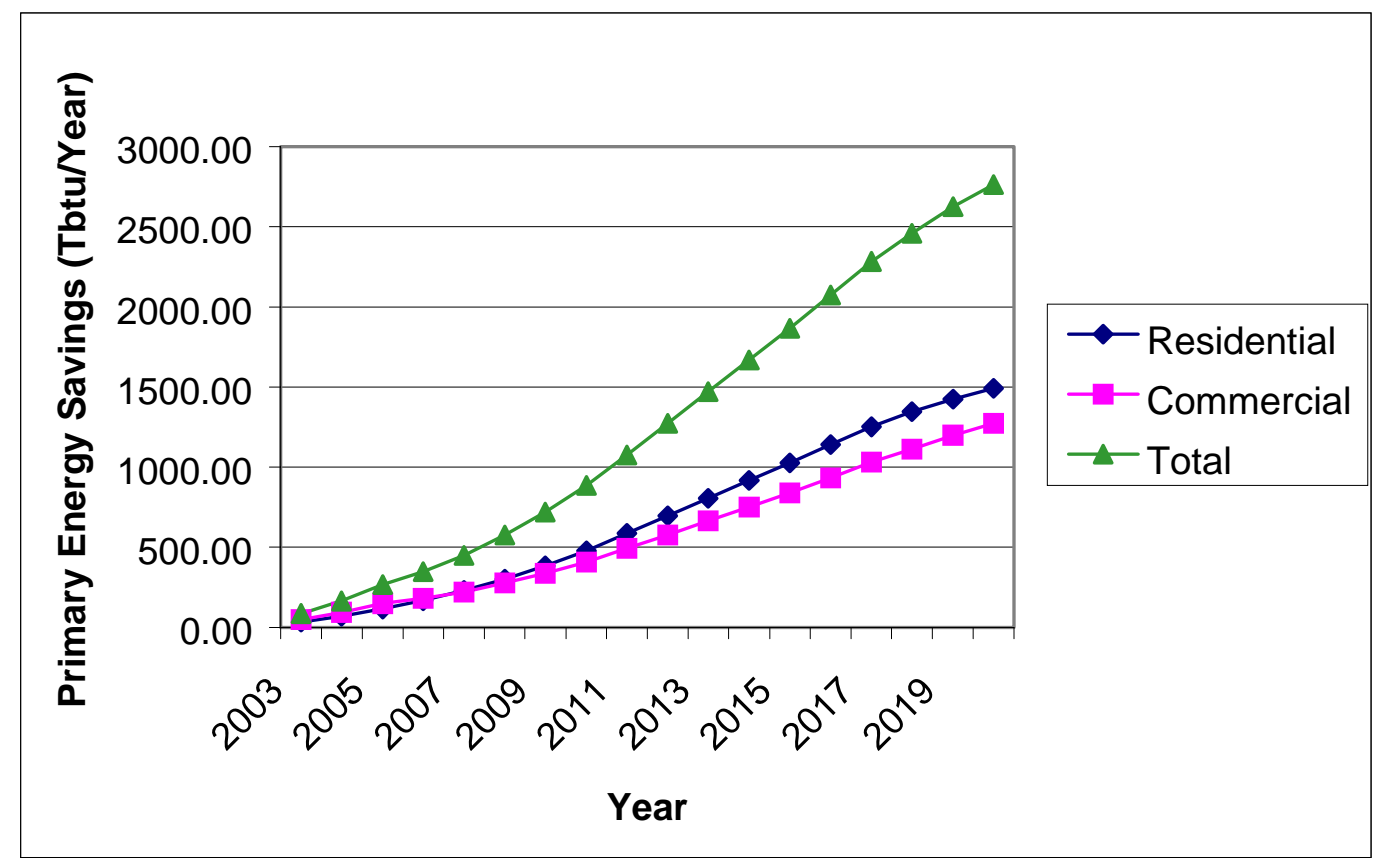

Figure 1. BTS Program Primary Energy Savings by Sector Through FY 2020

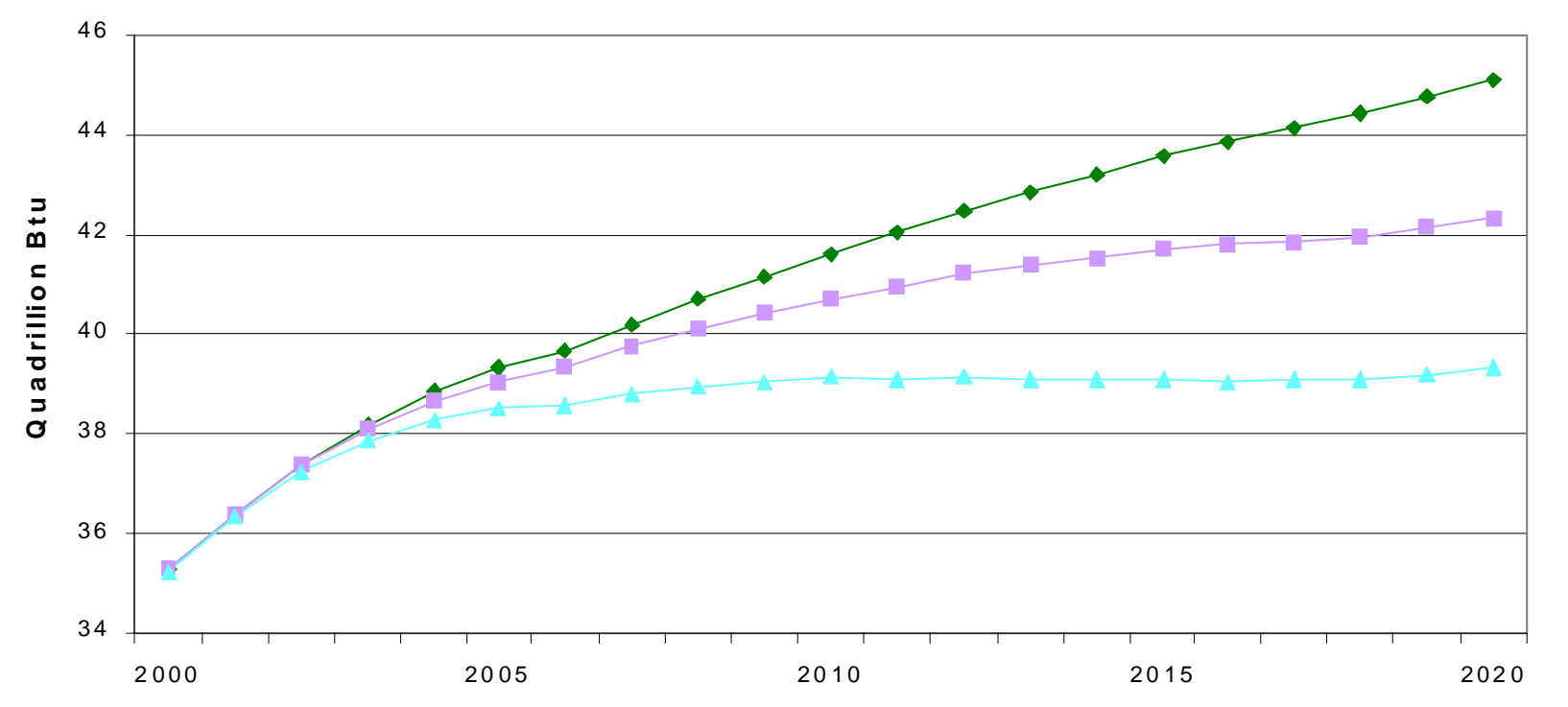

$\multimap$ Reference Case (AEO 2001) __FY $2003 \_$_ FY 2003 and Completed FY00, FY01 and FY02

Figure 2. Building Energy Consumption with and without BTS Savings 
Of all BTS energy savings (in year 2020), programs included in the Building Equipment and Materials decision unit generate $50 \%$ of the total savings (see Figure 3 ). This decision unit targets efficiency improvements for specific heating, cooling, and lighting equipment as well as shell (e.g., windows, roofs, and insulation) efficiency improvements, induding standards that impact specific equipment. Building Equipment and Materials makes up about $8 \%$ of the overall BTS program FY 2003 budget.

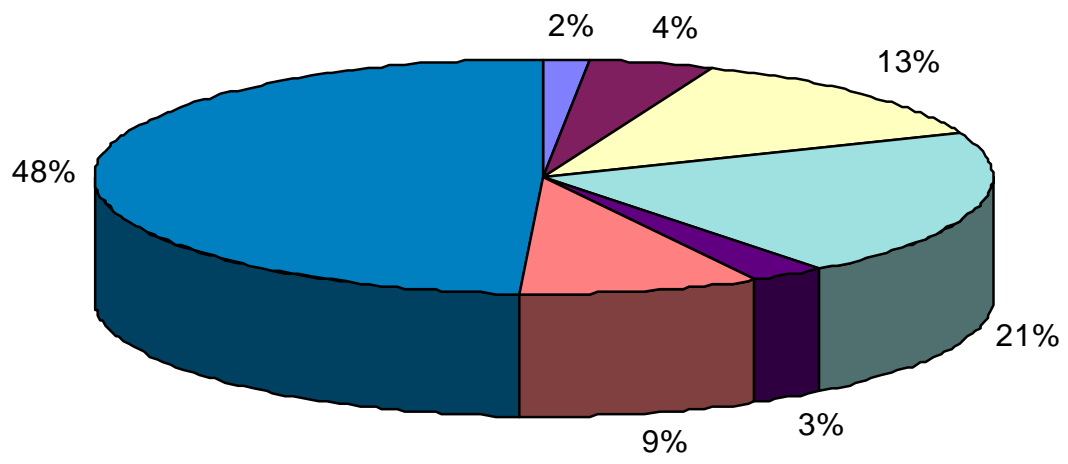

\begin{tabular}{|l}
$\square$ State Energy Program \\
$\square$ Weatherization \\
Assistance Program \\
$\square$ Community Energy \\
Program \\
$\square$ Energy Star Program \\
$\square$ Residential Buildings \\
Integration \\
$\square$ Commercial Buildings \\
Integration \\
$\square$ Building Equipment \& \\
Materials
\end{tabular}

Figure 3. Primary Energy Savings by Decision Unit (for 2020)

Energy Star makes up about $20 \%$ of the total savings while accounting for just under $2 \%$ of the total budget request. Programs that support the Community Energy Program make up an additional $13 \%$ of the overall BTS savings (in year 2020). Community Energy Programs indude a combination of programs that target whole-building energy use primarily by providing outreach, education, training and tools, and partnership assistance. Community Energy Programs make up 5\% of the overall BTS FY 2003 budget.

In terms of energy savings per budget dollar, the building codes programs and Energy Star have relatively high ratios of savings to budget dollar. The building codes programs benefit from having high penetration rates because these standards become regulatory mandates when adopted by states. Energy Star focuses on market transformation through labeling and requires relatively few dollars to implement compared with programs that provide R\&D or technical assistance. Programs such as Weatherization Assistance and State Energy programs tend to have relatively low ratios of savings to budget dollar because these programs provide grants and assistance directly to states and households. Figure 4 charts the FY 2003 budget dollars and the energy savings in 2020 of each decision unit. 


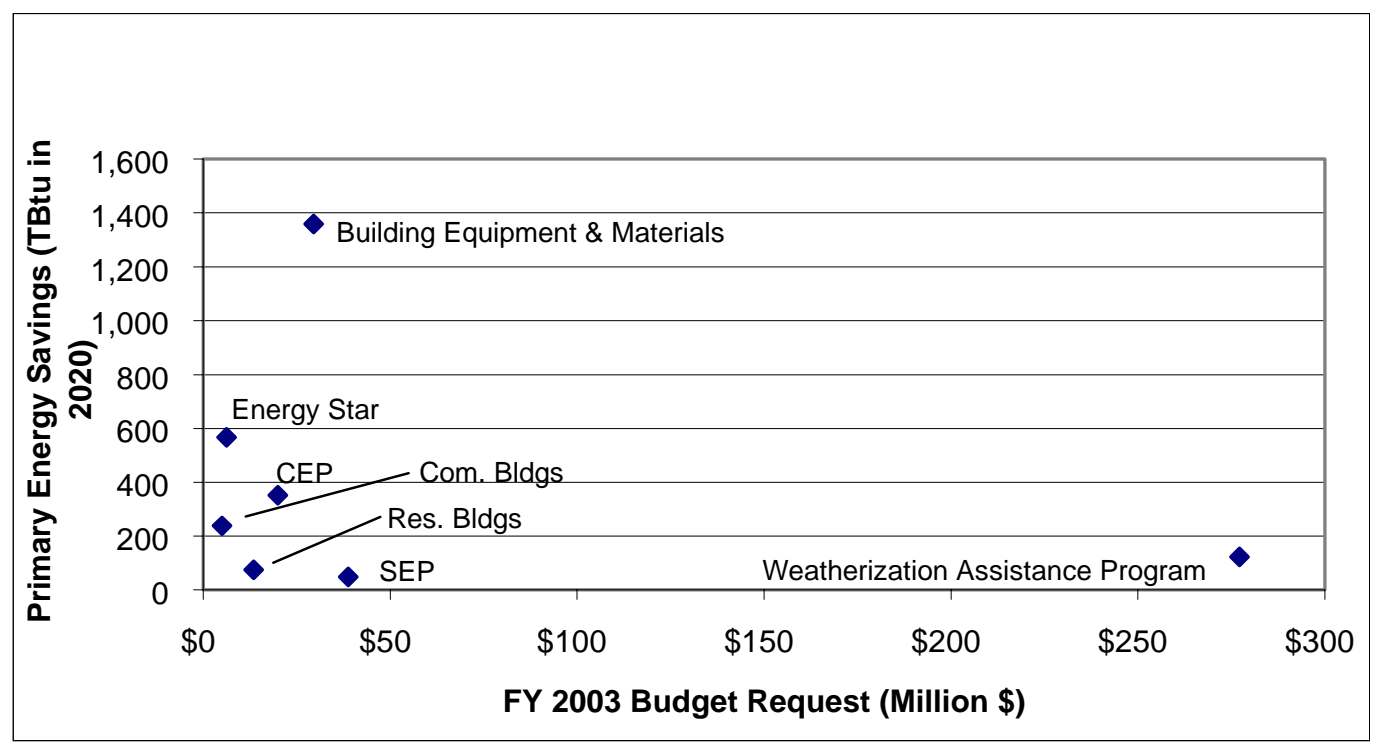

Figure 4. Budget and Energy Savings' Scatter Plot for BTS Decision Units 


\section{Detailed Results}




\section{FY 2003 GPRA Metrics for BTS Based on FY 2003 BTS Budget Request}

This section summarizes the GPRA metrics benefits estimates produced for BTS and submitted to OPBM as part of the FY 2003 EE GPRA metrics effort. These estimates were produced in conjunction with the final BTS FY 2003 budget request (dated 11/5/01), have been peer reviewed by Arthur D. Little, and represent the expected benefits as a result of program activities funded under the FY 2003 budget request. Benefits resulting from funding in years prior to FY 2003 are not included. Benefits accruing from future funding are included in the estimates.

\section{Comparison of FY 2001 Run 6 GPRA Metrics with FY 2003 Run 4N GPRA Metrics}

Energy savings' estimates are reported to OPBM at a decision unit level; however, this section will attempt to explain changes that arise at the individual program/technology level. While estimates at this level are not reported to OPBM as part of the GPRA metrics, they do provide a useful planning tool for BTS and variations should be understood.

Because the estimates produced for the FY 2002 GPRA effort were never issued by OPBM due to the change in Administration, the final FY 2003 GPRA results are compared with the results associated with the final FY 2001 request. Overall, the estimated energy savings from BTS programs through 2020 are lower in the early years than those associated with the FY 2001 request. The FY 2001 estimate of 1.3 QBtu in 2010 has fallen to 0.9 QBtu, while the FY 2001 estimate of primary savings in 2020 is essentially unchanged, decreasing very slightly from 2.78 QBtu to 2.76 QBtu.

The discussion has been divided into six categories relative to primary energy savings in the year 2020:

1. Programs characterized in FY 2001 but not in FY 2003

2. Programs characterized using the NAS methodology

3. Programs that did not change significantly

4. Programs that changed $25 \%$ to $50 \%$

5. Programs that changed more than $50 \%$

6. Programs characterized in FY 2003 but not in FY 2001.

Programs that fall into the first category have been completed or re-characterized and are not in the FY 2003 request. Programs in the second category were modeled assuming that the program accelerated the time to market. Programs that fall into the sixth category were not characterized in the FY 2001 metrics either because they were new to the FY 2003 request or not enough information was available about them in FY 2001 to characterize them.

The impact of these categories of programs is shown in the following table. 


\begin{tabular}{|l|l|}
\hline \multicolumn{1}{|c|}{ Changes by Category } & \multicolumn{1}{|c|}{$\begin{array}{c}\text { Year 2020 } \\
\text { (TBtu) }\end{array}$} \\
\hline FY 2001 Estimate & 2,781 \\
\hline Less & \\
\hline 1. Programs characterized in FY 2001 but not in FY 2003 & -551 \\
\hline Plus & \\
\hline 2. Programs characterized using the NAS methodology & 69 \\
\hline 3. Programs that have not changed significantly & 11 \\
\hline 4. Programs that have changed 25\% to 50\% & 29 \\
\hline 5. Programs with changes greater than 50\% & 60 \\
\hline 6. Programs characterized in FY 2003 but not in FY 2001 & 365 \\
\hline Equals & \\
\hline FY 2003 Estimate & 2,763 \\
\hline
\end{tabular}

\section{Programs Characterized in FY 2001 But Not in FY 2003}

Twelve BTS programs or technologies were characterized in FY 2001 but not in FY 2003:

- Energy Smart Schools: Estimated at 21 TBtu in 2020 (program now falls under Rebuild America)

- Competitively-Selected Community Projects: Estimated at 9 TBtu in 2020 (program now falls under Rebuild America)

- Partnership for Advancing Technology in Housing: Estimated at 167 TBtu in 2020

- Energy Star: Windows: Estimated at 56 TBtu in 2020 (activity transferred to the Building Envelope: Windows program)

- Competitive R\&D: Estimated at 162 TBtu in 2020

- Lighting R\&D: Advanced Light Sources, Electronics, and New Concepts: Estimated at 20 TBtu in 2020 (activity completed)

- Space Conditioning R\&D: GAX Heat Pump: Estimated at 31 TBtu in 2020 (program transferred to the Office of Power Technologies)

- Space Conditioning R\&D: Hi-Cool Heat Pump: Estimated at 19 TBtu in 2020 (program transferred to the Office of Power Technologies)

- Space Conditioning R\&D: Desiccants: Estimated at 16 TBtu in 2020 (program transferred to OPT)

- Cogeneration/Fuel Cells: Estimated at 37 TBtu in 2020 (program transferred to the Office of Power Technologies) 
- Appliances \& E merging Technologies: Compact Fluorescent Lights: Estimated at 5 TBtu in 2020 (activity completed)

- Appliances \& Emerging Technologies: Dryers: Estimated at 7 TBtu in 2020 (activity completed)

\section{Programs Characterized Using the NAS Methodology}

The calculation methodologies for the programs characterized using the NAS methodology were modified to remove the estimated benefits that would have occurred in the absence of DOE funding. This change was implemented within the BTS estimates by determining that the programs act as acceleration-to-market programs rather than programs that would not have been developed or implemented in the absence of government funding. This approach impacts the resulting BTS savings for the programs by diminishing the BTS savings in future years, presuming that the private sector is expanding its development and production of these technologies. Figure 1 illustrates how applying this acceleration methodology would impace a program's estimates in its most simplified state.

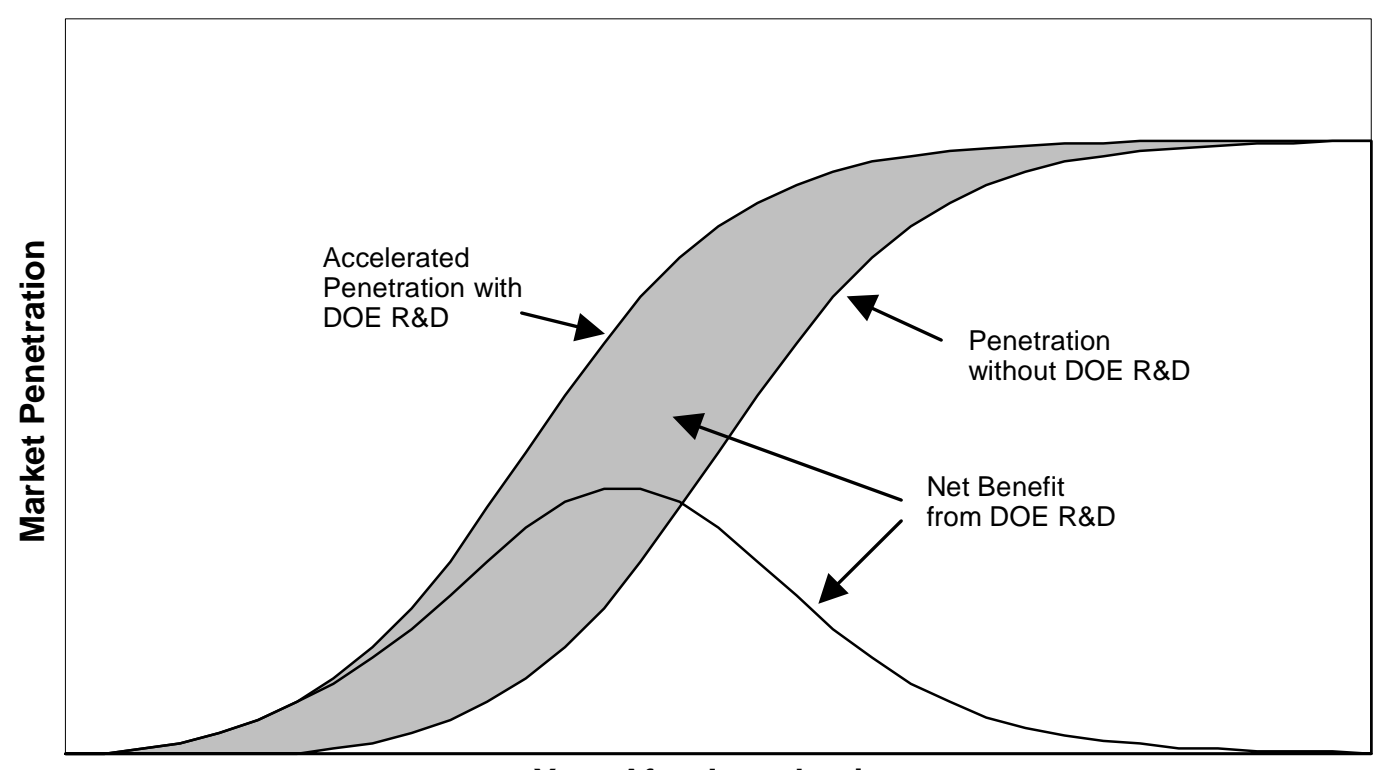

Year After Introduction

Figure 1. I mpact of the NAS Methodology (pure market acceleration case)

Six programs were characterizing using the NAS methodology:

- Information Outreach: Increased from 3 to 63 TBtu in 2020 because of a change in the modeling method. The FY 2001 estimates used an estimated savings per budget dollar of .0659 MBtu, which was based on the State Energy Program's analysis of utility demandside management information. The modeling method has been revised using an evaluation conducted for the Information Outreach program that estimated annual energy savings of 13.92 TBtu. The evaluation further assumed that savings lasted for three years, after which they were supplanted by activities the decision maker would have undertaken in any event. 
- Refrigeration \& Thermal Distribution R\&D: Commercial Refrigeration: Decreased from 52 to 22 TBtu in 2020. DOE involvement was assumed to accelerate the introduction of this technology by 10 years.

- Emerging Technologies R\&D: Heat Pump Water Heater: Increased from 53 TBtu to 65 TBtu in 2020. This is increase is the result of a change in the NEMS baseline and the characterization of competing technologies. DOE involvement was assumed to accelerate the introduction of this technology by 10 years.

- Emerging Technologies R\&D: Gas Condensing Water Heater: Increased from 4 to 25 TBtu in 2020. DOE involvement was assumed to accelerate the introduction of this technology by 10 years. Also, the cost parameters were changed to reflect more recent information. Previously, an incremental cost of between $\$ 330$ and $\$ 385$ had been assumed; for the FY 2003 effort, the incremental cost was assumed to be between $\$ 150$ and $\$ 200$.

- Emerging Technologies R\&D: Recessed Can Lights: Increased from 3 to 4 TBtu in 2020. DOE involvement was assumed to accelerate the introduction of this technology by 10 years. Also, this program was modeled in BESET for the FY 2003 effort, whereas it had been modeled in a spreadsheet for FY 2001.

- Building Envelope R\&D: Windows: Increased from 247 to 250 TBtu in 2020. DOE involvement was assumed to accelerate the introduction of this technology by 10 years. Also, the two windows technologies, electrochromics and superwindows, were modeled in NEMS for FY 2003 effort, whereas they had been modeled in a spreadsheet for the FY 2001.

\section{Programs That Did Not Change Significantly}

The modeling methods or characterizations for eight programs or technologies did not change significantly between FY 2001 and FY 2003:

- Training and Technical Assistance to Codes

- Energy Star: Refrigerators

- Energy Star: Electric Water Heaters

- Energy Star: Room Air Conditioners

- Commercial Building Codes

- Lighting R\&D: Two Photon Phosphors

- Design Strategies and Assistance

- Lighting and Appliance Standards.

\section{Programs That Changed $25 \%$ to $50 \%$}

Two programs had changes from $25 \%$ to $50 \%$ :

- Energy Star: Dishwashers: Decreased from 6 to 4 TBtu in 2020 because of a change in the NEMS baseline and the characterization of competing technologies. 
- Weatherization Assistance: Increased from 92 to 123 TBtu in 2020 because of the large increase in requested budget, which will allow more houses to be weatherized and a new strategy to be implemented that increases the expected savings per household.

\section{Programs That Changed More Than 50\%}

Nine programs had more than a $50 \%$ change:

- State Formula Grants: Decreased from 97 to 48 TBtu in 2020 because the forecasted mix of projects was revised. In FY 2001, the forecasted mix was based on the historical mix; after being externally peer-reviewed for the FY 2002 effort, the mix was adjusted to remove projects that were no longer being pursued.

- Rebuild America: Decreased from 83 to 26 TBtu in 2020 for two reasons: 1) the program is scheduled to end in 2010 and the new estimates exclude the FY 2001 and FY 2002 floor space retrofitted and 2) previously, the $25 \%$ and $40 \%$ energy use reductions were assumed to be whole-building reductions, which were modeled as $44 \%$ and $71 \%$ reductions in space conditioning and water heating, respectively. Based on revised information from the Program Manager, the $25 \%$ and $40 \%$ reductions were assumed to apply only to space conditioning.

- Energy Star: Clothes Washers: Increased from 33 to 64 TBtu because of a change in the NEMS baseline and the characterization of competing technologies.

- Energy Star: Compact Fluorescent Lights: Increased from 93 to 419 TBtu in 2020 primarily because of significant baseline changes in residential lighting loads. Between FY 2001 and FY 2003, the weighted average of the baseline residential lighting loads increased by over $100 \%$ for all vintages and climates zones. In addition, the underlying algorithm on which these estimates were based was modified.

- Residential Technology R\&D: Decreased from 106 to 21 TBtu in 2020 because of revisions in the number of households impacted each year.

- Residential Building Codes: Increased from 5 to 53 TBtu in 2020 because of a change in the assumptions on stringency of the IECC code and a future new standard.

- Commercial Technology Development: Increased from 53 to 139 TBtu in 2020 because of revisions in the program goals based on the High-Performance Commercial Buildings Roadmap.

- Emerging Technologies R\&D: Roof Top Air Conditioning: Decreased from 8 to 3 TBtu in 2020 because of a change in the NEMS baseline and the characterization of competing technologies.

- Building Envelope R\&D: Thermal Insulation and Building Materials: Decreased from 297 to 62 TBtu in 2020 because of revisions in the technologies modeled. For FY 
2001, the program was represented by advanced insulations and R30 insulation/30-year life roofs; for FY 2003, the program was represented by quick fill walls, R30 insulation/ 30-year life roofs, and moisture/wet insulation technologies.

\section{Programs Characterized in FY 2003 But Not in FY 2001}

Some programs were added to the BTS GPRA characterization in FY 2003. While some of the programs are new to the BTS portfolio, others have been added either because they represent a line item within the 2003 budget request (but did not in FY 2001) or because not enough information was available in FY 2001 to adequately characterize them.

In general, programs are included in GPRA based on whether they are a line item in the initial budget request with a specific funding allocation. Occasionally, programs do not appear as a line item in the initial funding request but do appear in the final request. These programs are characterized within GPRA metrics only if enough information is available to characterize the program in a short period of time. In the case of programs covering a suite of technologies, the technologies characterized are based on discussions with the Program Manager. This suite of technologies may change from year to year as the Program Manager changes focus. The suite of technologies is meant to be representative of the program; it is not meant to capture all funded activities.

Four programs were characterized in FY 2003 but not in FY 2001:

- Energy Star: Gas Water Heaters: Estimated at 11 TBtu in 2020.

- Lighting R\&D:

- Solid State Lighting: Estimated at 49 TBtu in 2020.

- Lighting Controls: Estimated at 40 TBtu in 2020.

- Refrigeration and Thermal Distribution R\&D:

- Residential HVAC Distribution System: Estimated at 102 TBtu in 2020 (this program also applied the NAS methodology, assuming that DOE involvement accelerated the introduction of this technology by 10 years).

- Advanced Electric Heat Pump Water Heater: Estimated at 48 TBtu in 2020 (this program also applied the NAS methodology, assuming that DOE involvement accelerated the introduction of this technology by 10 years).

- Refrigerant Meter: Estimated at 117 TBtu in 2020 (this program also applied the NAS methodology, assuming that DOE involvement accelerated the introduction of this technology by 10 years).

- Emerging Technologies R\&D: R-Lamp: Estimated at 0 TBtu in 2020. This program applied the NAS methodology, assuming that DOE involvement accelerated the introduction of this technology by 10 years. However, because of the short life span of the technology, the impacts occur earlier, so that by 2020, not DOE impacts are recorded (see Figure 1). The peak impact is around 2010, with an estimated impact of 31 TBtu. 


\section{FY 2003 GPRA Metrics ñ Detailed Tables}

At the end of this section tables are shown that include forecasted benefits up to the year 2030 for all programs and decision units. The benefit estimates available include the following:

- Energy Savings Benefits Tables (TBtu/yr)

- Total Primary Energy Savings

- Primary Electricity savings

- Primary Non-Electric Savings

- Site Electricity Savings

- Site Natural Gas Savings

- Site Oil Savings

- Environmental Benefits Tables (million metric tons (MMT)/yr)

- Carbon Equivalent Emissions Reductions

- $\mathrm{SO}_{2}$ Emissions Reductions

- $\mathrm{NO}_{x}$ Emissions Reductions

- CO Emissions Reductions

- PM Emissions Reductions

- VOC Emissions Reductions

- Financial Benefits Tables (million $\$ / y r$ )

- Consumer Cost Savings

- Non-Energy Cost Savings.

In all benefits tables, program benefits are reported annually. The energy savings' estimate for 2010 represents energy saved in 2010 only. These are not cumulative benefits estimates. However, the energy saved in 2010 is a function of all program activities from FY 2003 on, so that the number of affected buildings is a cumulative value. For example, the energy saved in 2010 from the compact fluorescent lights programs is the energy saved in 2010 only, from all buildings that have had such lights installed any time between FY 2003 and FY 2010.

Reductions in emissions from BTS programs are calculated from GPRA metrics estimates of energy savings by fuel type, multiplied by emissions coefficients provided by OPBM for use in GPRA metrics.

The consumer cost savings' estimates are calculated from GPRA metrics estimates of energy savings by sector and fuel type, multiplied by energy price forecasts provided by OPBM for use in GPRA metrics. 


\section{Carbon Equivalent Emissions Reductions}


Carbon Equivalent Emissions Reductions (MMtons/yr)

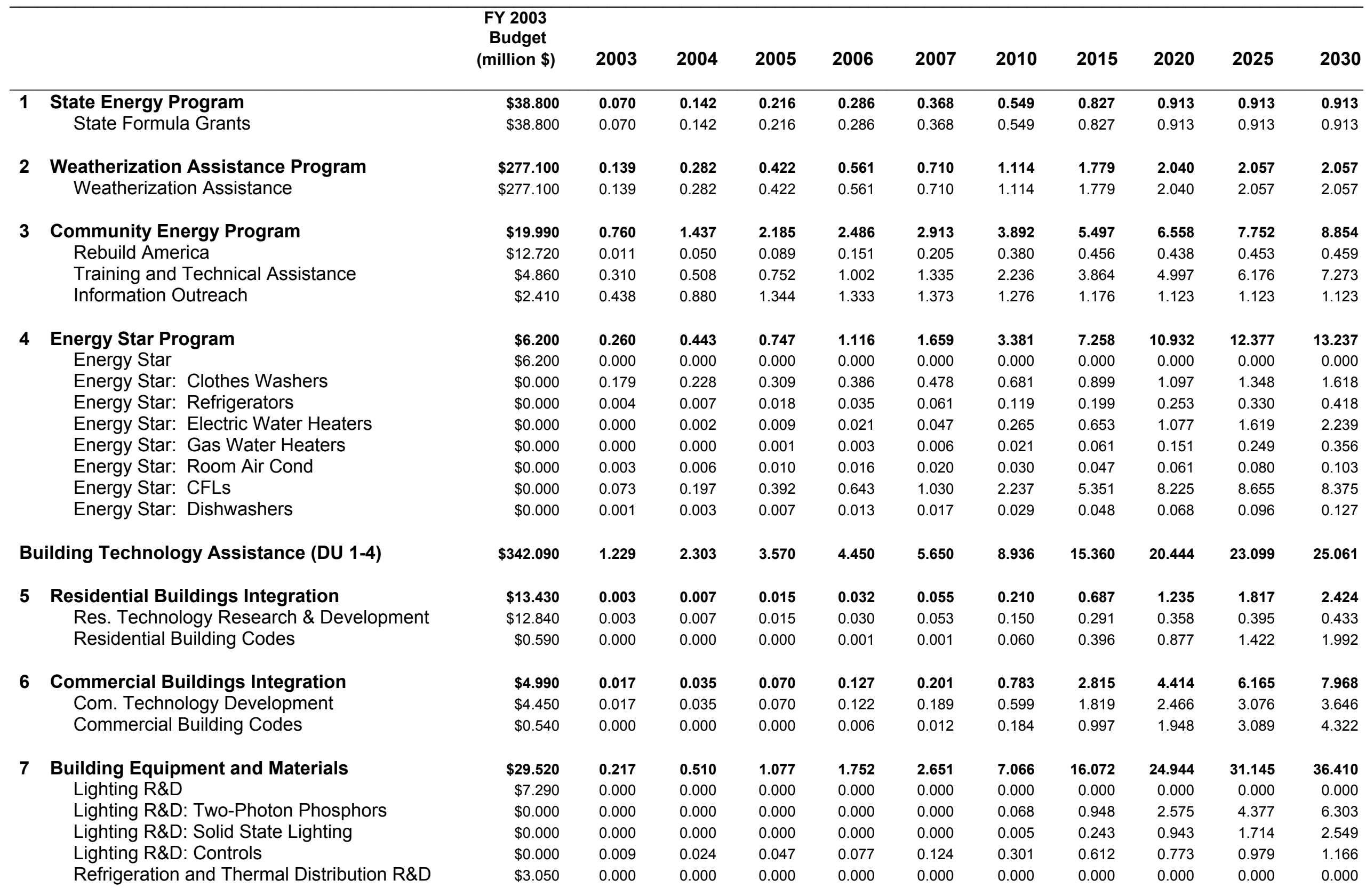

Run Title: FY 2003 Run 4 NAS

MDB: $\quad K: I M E T R I C S I F Y 2003 \mid B E S E T$ InputsIRun 4 NASIFY 2003 Run 4 NAS.mdb

Scenario Last Executed: $12 / 14 / 01 \quad 1: 24: 50$ PM 
Carbon Equivalent Emissions Reductions (MMtons/yr)

\begin{tabular}{|c|c|c|c|c|c|c|c|c|c|c|c|}
\hline & $\begin{array}{c}\text { FY } 2003 \\
\text { Budget } \\
\text { (million \$) }\end{array}$ & 2003 & 2004 & 2005 & 2006 & 2007 & 2010 & 2015 & 2020 & 2025 & 2030 \\
\hline $\begin{array}{l}\text { Refrig. \& Thermal Dist. R\&D: Res. HVAC } \\
\text { Dist. System }\end{array}$ & $\$ 0.000$ & 0.025 & 0.062 & 0.116 & 0.190 & 0.300 & 0.818 & 1.849 & 1.683 & 0.831 & 0.593 \\
\hline $\begin{array}{l}\text { Refrig. \& Thermal Dist. R\&D: Adv. Elec } \\
\text { HPWH }\end{array}$ & $\$ 0.000$ & 0.000 & 0.000 & 0.002 & 0.007 & 0.016 & 0.073 & 0.398 & 0.925 & 1.118 & 0.679 \\
\hline $\begin{array}{l}\text { Refrig. \& Thermal Dist. R\&D: Commercial } \\
\text { Refrigeration }\end{array}$ & $\$ 0.000$ & 0.024 & 0.056 & 0.097 & 0.143 & 0.207 & 0.389 & 0.569 & 0.425 & 0.193 & 0.062 \\
\hline $\begin{array}{l}\text { Refrig. \& Thermal Dist. R\&D: Refrigerant } \\
\text { Meter }\end{array}$ & $\$ 0.000$ & 0.000 & 0.000 & 0.005 & 0.017 & 0.040 & 0.199 & 1.039 & 2.268 & 2.606 & 1.485 \\
\hline Emerging Technologies R\&D & $\$ 1.930$ & 0.000 & 0.000 & 0.000 & 0.000 & 0.000 & 0.000 & 0.000 & 0.000 & 0.000 & 0.000 \\
\hline Emerging Tech R\&D: HPWH & $\$ 0.000$ & 0.000 & 0.038 & 0.063 & 0.102 & 0.168 & 0.503 & 1.104 & 1.268 & 1.536 & 1.838 \\
\hline Emerging Tech R\&D: Roof Top AC & $\$ 0.000$ & 0.000 & 0.000 & 0.012 & 0.022 & 0.032 & 0.048 & 0.060 & 0.066 & 0.077 & 0.089 \\
\hline Emerging Tech R\&D: Gas Condensing WH & $\$ 0.000$ & 0.000 & 0.000 & 0.003 & 0.008 & 0.017 & 0.101 & 0.302 & 0.365 & 0.433 & 0.507 \\
\hline Emerging Tech R\&D: Recessed Can Lights & $\$ 0.000$ & 0.001 & 0.003 & 0.005 & 0.008 & 0.013 & 0.032 & 0.066 & 0.076 & 0.076 & 0.076 \\
\hline Emerging Tech R\&D: R-Lamp & $\$ 0.000$ & 0.035 & 0.094 & 0.187 & 0.307 & 0.423 & 0.646 & 0.084 & 0.000 & 0.000 & 0.000 \\
\hline Envelope R\&D: Electrochromic Windows & $\$ 0.000$ & 0.072 & 0.111 & 0.167 & 0.228 & 0.307 & 0.504 & 0.777 & 1.110 & 1.537 & 2.009 \\
\hline Envelope R\&D: Superwindows & $\$ 0.000$ & 0.039 & 0.091 & 0.160 & 0.242 & 0.352 & 0.806 & 2.063 & 3.240 & 4.575 & 6.031 \\
\hline Bldg. Env. R\&D: Roofs and Insulation & $\$ 1.560$ & 0.000 & 0.000 & 0.000 & 0.000 & 0.000 & 0.000 & 0.000 & 0.000 & 0.000 & 0.000 \\
\hline Envelope R\&D: Quick Fill Walls & $\$ 0.000$ & 0.000 & 0.000 & 0.001 & 0.002 & 0.003 & 0.009 & 0.035 & 0.079 & 0.129 & 0.183 \\
\hline $\begin{array}{l}\text { Envelope R\&D: R30 Insulation/30 Year } \\
\text { Life Roofs }\end{array}$ & $\$ 0.000$ & 0.000 & 0.000 & 0.000 & 0.000 & 0.000 & 0.005 & 0.069 & 0.220 & 0.377 & 0.539 \\
\hline Envelope R\&D: Moisture/Wet Insulation & $\$ 0.000$ & 0.000 & 0.003 & 0.012 & 0.024 & 0.041 & 0.122 & 0.335 & 0.648 & 1.002 & 1.393 \\
\hline Design Strategies and Assistance & $\$ 3.130$ & 0.011 & 0.028 & 0.054 & 0.087 & 0.134 & 0.309 & 0.679 & 0.971 & 1.348 & 1.721 \\
\hline Standards & $\$ 8.930$ & 0.000 & 0.000 & 0.000 & 0.000 & 0.000 & 0.000 & 0.000 & 0.000 & 0.000 & 0.000 \\
\hline Standards: Res Gas Furnaces/Boilers & $\$ 0.000$ & 0.000 & 0.000 & 0.000 & 0.000 & 0.000 & 0.068 & 0.250 & 0.444 & 0.658 & 0.893 \\
\hline Standards: EPACT Standards & $\$ 0.000$ & 0.000 & 0.000 & 0.145 & 0.288 & 0.474 & 1.687 & 3.706 & 5.527 & 6.240 & 6.954 \\
\hline Standards: Dist. Transformers & $\$ 0.000$ & 0.000 & 0.000 & 0.000 & 0.000 & 0.000 & 0.373 & 0.886 & 1.339 & 1.339 & 1.339 \\
\hline Building Research and Standards (DU 5-7) & $\$ 47.940$ & 0.237 & 0.552 & 1.163 & 1.912 & 2.907 & 8.060 & 19.574 & 30.592 & 39.127 & 46.802 \\
\hline All BTS & $\$ 390.030$ & 1.466 & 2.855 & 4.733 & 6.361 & 8.558 & 16.996 & 34.934 & 51.036 & 62.226 & 71.864 \\
\hline
\end{tabular}

Run Title: FY 2003 Run 4 NAS

Scenario Last Executed: $12 / 14 / 01 \quad 1: 24: 50$ PM

MDB: $\quad$ K:IMETRICSIFY2003IBESET InputsIRun 4 NASIFY 2003 Run 4 NAS.mdb 


\section{CO Emissions Reductions}


CO Emissions Reductions (MMTons/yr)

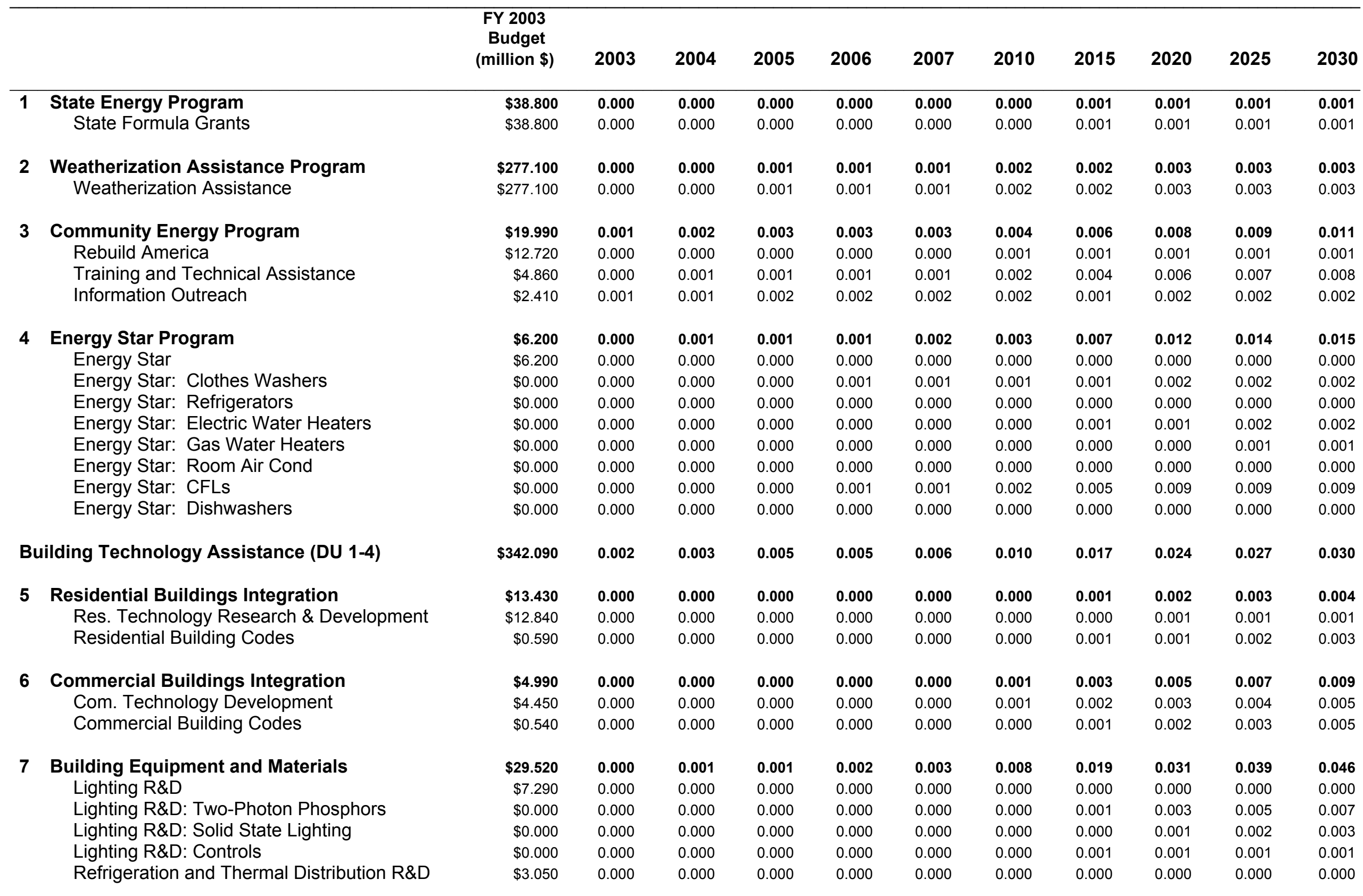

Run Title: FY 2003 Run 4 NAS

MDB: K:IMETRICSIFY2003IBESET InputsIRun 4 NASIFY 2003 Run 4 NAS.mdb

Scenario Last Executed: $12 / 14 / 01 \quad 1: 24: 50$ PM 
CO Emissions Reductions (MMTons/yr)

\begin{tabular}{|c|c|c|c|c|c|c|c|c|c|c|c|}
\hline & $\begin{array}{c}\text { FY } 2003 \\
\text { Budget } \\
\text { (million \$) }\end{array}$ & 2003 & 2004 & 2005 & 2006 & 2007 & 2010 & 2015 & 2020 & 2025 & 2030 \\
\hline $\begin{array}{l}\text { Refrig. \& Thermal Dist. R\&D: Res. HVAC } \\
\text { Dist. System }\end{array}$ & $\$ 0.000$ & 0.000 & 0.000 & 0.000 & 0.000 & 0.000 & 0.001 & 0.003 & 0.003 & 0.001 & 0.001 \\
\hline $\begin{array}{l}\text { Refrig. \& Thermal Dist. R\&D: Adv. Elec } \\
\text { HPWH }\end{array}$ & $\$ 0.000$ & 0.000 & 0.000 & 0.000 & 0.000 & 0.000 & 0.000 & 0.000 & 0.001 & 0.001 & 0.001 \\
\hline $\begin{array}{l}\text { Refrig. \& Thermal Dist. R\&D: Commercial } \\
\text { Refrigeration }\end{array}$ & $\$ 0.000$ & 0.000 & 0.000 & 0.000 & 0.000 & 0.000 & 0.000 & 0.001 & 0.000 & 0.000 & 0.000 \\
\hline $\begin{array}{l}\text { Refrig. \& Thermal Dist. R\&D: Refrigerant } \\
\text { Meter }\end{array}$ & $\$ 0.000$ & 0.000 & 0.000 & 0.000 & 0.000 & 0.000 & 0.000 & 0.001 & 0.002 & 0.003 & 0.002 \\
\hline Emerging Technologies R\&D & $\$ 1.930$ & 0.000 & 0.000 & 0.000 & 0.000 & 0.000 & 0.000 & 0.000 & 0.000 & 0.000 & 0.000 \\
\hline Emerging Tech R\&D: HPWH & $\$ 0.000$ & 0.000 & 0.000 & 0.000 & 0.000 & 0.000 & 0.000 & 0.001 & 0.001 & 0.002 & 0.002 \\
\hline Emerging Tech R\&D: Roof Top AC & $\$ 0.000$ & 0.000 & 0.000 & 0.000 & 0.000 & 0.000 & 0.000 & 0.000 & 0.000 & 0.000 & 0.000 \\
\hline Emerging Tech R\&D: Gas Condensing WH & $\$ 0.000$ & 0.000 & 0.000 & 0.000 & 0.000 & 0.000 & 0.000 & 0.001 & 0.001 & 0.001 & 0.001 \\
\hline Emerging Tech R\&D: Recessed Can Lights & $\$ 0.000$ & 0.000 & 0.000 & 0.000 & 0.000 & 0.000 & 0.000 & 0.000 & 0.000 & 0.000 & 0.000 \\
\hline Emerging Tech R\&D: R-Lamp & $\$ 0.000$ & 0.000 & 0.000 & 0.000 & 0.000 & 0.000 & 0.001 & 0.000 & 0.000 & 0.000 & 0.000 \\
\hline Envelope R\&D: Electrochromic Windows & $\$ 0.000$ & 0.000 & 0.000 & 0.000 & 0.000 & 0.000 & 0.000 & 0.001 & 0.001 & 0.002 & 0.002 \\
\hline Envelope R\&D: Superwindows & $\$ 0.000$ & 0.000 & 0.000 & 0.000 & 0.000 & 0.000 & 0.001 & 0.003 & 0.005 & 0.007 & 0.009 \\
\hline Bldg. Env. R\&D: Roofs and Insulation & $\$ 1.560$ & 0.000 & 0.000 & 0.000 & 0.000 & 0.000 & 0.000 & 0.000 & 0.000 & 0.000 & 0.000 \\
\hline Envelope R\&D: Quick Fill Walls & $\$ 0.000$ & 0.000 & 0.000 & 0.000 & 0.000 & 0.000 & 0.000 & 0.000 & 0.000 & 0.000 & 0.000 \\
\hline $\begin{array}{l}\text { Envelope R\&D: R30 Insulation/30 Year } \\
\text { Life Roofs }\end{array}$ & $\$ 0.000$ & 0.000 & 0.000 & 0.000 & 0.000 & 0.000 & 0.000 & 0.000 & 0.000 & 0.001 & 0.001 \\
\hline Envelope R\&D: Moisture/Wet Insulation & $\$ 0.000$ & 0.000 & 0.000 & 0.000 & 0.000 & 0.000 & 0.000 & 0.001 & 0.001 & 0.002 & 0.002 \\
\hline Design Strategies and Assistance & $\$ 3.130$ & 0.000 & 0.000 & 0.000 & 0.000 & 0.000 & 0.000 & 0.001 & 0.001 & 0.002 & 0.002 \\
\hline Standards & $\$ 8.930$ & 0.000 & 0.000 & 0.000 & 0.000 & 0.000 & 0.000 & 0.000 & 0.000 & 0.000 & 0.000 \\
\hline Standards: Res Gas Furnaces/Boilers & $\$ 0.000$ & 0.000 & 0.000 & 0.000 & 0.000 & 0.000 & 0.000 & 0.001 & 0.001 & 0.001 & 0.002 \\
\hline Standards: EPACT Standards & $\$ 0.000$ & 0.000 & 0.000 & 0.000 & 0.000 & 0.000 & 0.002 & 0.004 & 0.006 & 0.007 & 0.008 \\
\hline Standards: Dist. Transformers & $\$ 0.000$ & 0.000 & 0.000 & 0.000 & 0.000 & 0.000 & 0.000 & 0.001 & 0.001 & 0.001 & 0.001 \\
\hline Building Research and Standards (DU 5-7) & $\$ 47.940$ & 0.000 & 0.001 & 0.001 & 0.002 & 0.003 & 0.009 & 0.023 & 0.038 & 0.049 & 0.059 \\
\hline All BTS & $\$ 390.030$ & 0.002 & 0.004 & 0.006 & 0.008 & 0.009 & 0.018 & 0.040 & 0.062 & 0.076 & 0.088 \\
\hline
\end{tabular}

Run Title: FY 2003 Run 4 NAS

Scenario Last Executed: $12 / 14 / 01 \quad 1: 24: 50$ PM

MDB: $\quad$ K:IMETRICSIFY2003IBESET InputsIRun 4 NASIFY 2003 Run 4 NAS.mdb 


\section{Consumer Cost Savings}


Consumer Cost Savings (million \$/yr)

\begin{tabular}{|c|c|c|c|c|c|c|c|c|c|c|c|c|}
\hline & & $\begin{array}{c}\text { FY } 2003 \\
\text { Budget } \\
\text { (million \$) }\end{array}$ & 2003 & 2004 & 2005 & 2006 & 2007 & 2010 & 2015 & 2020 & 2025 & 2030 \\
\hline \multirow[t]{2}{*}{1} & State Energy Program & $\$ 38.800$ & $\$ 24$ & $\$ 48$ & $\$ 70$ & $\$ 92$ & $\$ 113$ & $\$ 177$ & $\$ 292$ & $\$ 345$ & $\$ 352$ & $\$ 359$ \\
\hline & State Formula Grants & $\$ 38.800$ & $\$ 24$ & $\$ 48$ & $\$ 70$ & $\$ 92$ & $\$ 113$ & $\$ 177$ & $\$ 292$ & $\$ 345$ & $\$ 352$ & $\$ 359$ \\
\hline \multirow[t]{2}{*}{2} & Weatherization Assistance Program & $\$ 277.100$ & $\$ 59$ & $\$ 118$ & $\$ 175$ & $\$ 232$ & $\$ 291$ & $\$ 469$ & $\$ 774$ & $\$ 917$ & $\$ 934$ & $\$ 944$ \\
\hline & Weatherization Assistance & $\$ 277.100$ & $\$ 59$ & $\$ 118$ & $\$ 175$ & $\$ 232$ & $\$ 291$ & $\$ 469$ & $\$ 774$ & $\$ 917$ & $\$ 934$ & $\$ 944$ \\
\hline \multirow[t]{4}{*}{3} & Community Energy Program & $\$ 19.990$ & $\$ 281$ & $\$ 522$ & $\$ 756$ & $\$ 856$ & $\$ 947$ & $\$ 1,344$ & $\$ 2,107$ & $\$ 2,723$ & $\$ 3,259$ & $\$ 3,771$ \\
\hline & Rebuild America & $\$ 12.720$ & $\$ 5$ & $\$ 20$ & $\$ 34$ & $\$ 58$ & $\$ 76$ & $\$ 148$ & $\$ 190$ & $\$ 193$ & $\$ 202$ & $\$ 207$ \\
\hline & Training and Technical Assistance & $\$ 4.860$ & $\$ 116$ & $\$ 186$ & $\$ 259$ & $\$ 342$ & $\$ 424$ & $\$ 759$ & $\$ 1,478$ & $\$ 2,079$ & $\$ 2,600$ & $\$ 3,100$ \\
\hline & Information Outreach & $\$ 2.410$ & $\$ 161$ & $\$ 317$ & $\$ 464$ & $\$ 456$ & $\$ 447$ & $\$ 437$ & $\$ 439$ & $\$ 451$ & $\$ 457$ & $\$ 464$ \\
\hline \multirow[t]{9}{*}{4} & Energy Star Program & $\$ 6.200$ & $\$ 107$ & $\$ 178$ & $\$ 288$ & $\$ 436$ & $\$ 618$ & $\$ 1,375$ & $\$ 3,312$ & $\$ 5,373$ & $\$ 6,118$ & $\$ 6,578$ \\
\hline & Energy Star & $\$ 6.200$ & $\$ 0$ & $\$ 0$ & $\$ 0$ & $\$ 0$ & $\$ 0$ & $\$ 0$ & $\$ 0$ & $\$ 0$ & $\$ 0$ & $\$ 0$ \\
\hline & Energy Star: Clothes Washers & $\$ 0.000$ & $\$ 75$ & $\$ 94$ & $\$ 124$ & $\$ 156$ & $\$ 188$ & $\$ 285$ & $\$ 404$ & $\$ 519$ & $\$ 641$ & $\$ 774$ \\
\hline & Energy Star: Refrigerators & $\$ 0.000$ & $\$ 2$ & $\$ 3$ & $\$ 7$ & $\$ 13$ & $\$ 22$ & $\$ 48$ & $\$ 91$ & $\$ 125$ & $\$ 164$ & $\$ 209$ \\
\hline & Energy Star: Electric Water Heaters & $\$ 0.000$ & $\$ 0$ & $\$ 1$ & $\$ 4$ & $\$ 8$ & $\$ 17$ & $\$ 107$ & $\$ 299$ & $\$ 531$ & $\$ 804$ & $\$ 1,119$ \\
\hline & Energy Star: Gas Water Heaters & $\$ 0.000$ & $\$ 0$ & $\$ 0$ & $\$ 0$ & $\$ 1$ & $\$ 3$ & $\$ 10$ & $\$ 27$ & $\$ 69$ & $\$ 113$ & $\$ 162$ \\
\hline & Energy Star: Room Air Cond & $\$ 0.000$ & $\$ 1$ & $\$ 2$ & $\$ 4$ & $\$ 6$ & $\$ 7$ & $\$ 12$ & $\$ 21$ & $\$ 30$ & $\$ 40$ & $\$ 52$ \\
\hline & Energy Star: CFLs & $\$ 0.000$ & $\$ 29$ & $\$ 77$ & $\$ 147$ & $\$ 246$ & $\$ 375$ & $\$ 902$ & $\$ 2,448$ & $\$ 4,066$ & $\$ 4,308$ & $\$ 4,198$ \\
\hline & Energy Star: Dishwashers & $\$ 0.000$ & $\$ 0$ & $\$ 1$ & $\$ 3$ & $\$ 5$ & $\$ 6$ & $\$ 12$ & $\$ 22$ & $\$ 34$ & $\$ 48$ & $\$ 63$ \\
\hline \multicolumn{2}{|r|}{ Building Technology Assistance (DU 1-4) } & $\$ 342.090$ & $\$ 471$ & $\$ 867$ & $\$ 1,289$ & $\$ 1,616$ & $\$ 1,969$ & $\$ 3,365$ & $\$ 6,485$ & $\$ 9,358$ & $\$ 10,663$ & $\$ 11,652$ \\
\hline \multirow[t]{3}{*}{5} & Residential Buildings Integration & $\$ 13.430$ & $\$ 1$ & $\$ 3$ & $\$ 6$ & $\$ 13$ & $\$ 22$ & $\$ 89$ & $\$ 307$ & $\$ 575$ & $\$ 851$ & $\$ 1,142$ \\
\hline & Res. Technology Research \& Development & $\$ 12.840$ & $\$ 1$ & $\$ 3$ & $\$ 6$ & $\$ 13$ & $\$ 22$ & $\$ 64$ & $\$ 131$ & $\$ 169$ & $\$ 188$ & $\$ 207$ \\
\hline & Residential Building Codes & $\$ 0.590$ & $\$ 0$ & $\$ 0$ & $\$ 0$ & $\$ 1$ & $\$ 1$ & $\$ 26$ & $\$ 176$ & $\$ 406$ & $\$ 663$ & $\$ 936$ \\
\hline \multirow[t]{3}{*}{6} & Commercial Buildings Integration & $\$ 4.990$ & $\$ 6$ & $\$ 13$ & $\$ 25$ & $\$ 45$ & $\$ 68$ & $\$ 275$ & $\$ 1,078$ & $\$ 1,820$ & $\$ 2,571$ & $\$ 3,363$ \\
\hline & Com. Technology Development & $\$ 4.450$ & $\$ 6$ & $\$ 13$ & $\$ 25$ & $\$ 43$ & $\$ 64$ & $\$ 215$ & $\$ 711$ & $\$ 1,035$ & $\$ 1,309$ & $\$ 1,573$ \\
\hline & Commercial Building Codes & $\$ 0.540$ & $\$ 0$ & $\$ 0$ & $\$ 0$ & $\$ 2$ & $\$ 4$ & $\$ 60$ & $\$ 367$ & $\$ 785$ & $\$ 1,262$ & $\$ 1,791$ \\
\hline \multirow[t]{5}{*}{7} & Building Equipment and Materials & $\$ 29.520$ & $\$ 84$ & $\$ 197$ & $\$ 395$ & $\$ 643$ & $\$ 925$ & $\$ 2,612$ & $\$ 6,510$ & $\$ 10,792$ & $\$ 13,556$ & $\$ 15,904$ \\
\hline & Lighting R\&D & $\$ 7.290$ & $\$ 0$ & $\$ 0$ & $\$ 0$ & $\$ 0$ & $\$ 0$ & $\$ 0$ & $\$ 0$ & $\$ 0$ & $\$ 0$ & $\$ 0$ \\
\hline & Lighting R\&D: Two-Photon Phosphors & $\$ 0.000$ & $\$ 0$ & $\$ 0$ & $\$ 0$ & $\$ 0$ & $\$ 0$ & $\$ 22$ & $\$ 349$ & $\$ 1,038$ & $\$ 1,788$ & $\$ 2,611$ \\
\hline & Lighting R\&D: Solid State Lighting & $\$ 0.000$ & $\$ 0$ & $\$ 0$ & $\$ 0$ & $\$ 0$ & $\$ 0$ & $\$ 2$ & $\$ 89$ & $\$ 380$ & $\$ 700$ & $\$ 1,056$ \\
\hline & Lighting R\&D: Controls & $\$ 0.000$ & $\$ 3$ & $\$ 9$ & $\$ 16$ & $\$ 26$ & $\$ 38$ & $\$ 98$ & $\$ 225$ & $\$ 312$ & $\$ 400$ & $\$ 483$ \\
\hline
\end{tabular}

Run Title: FY 2003 Run 4 NAS

MDB: $\quad K: I M E T R I C S I F Y 2003 \mid B E S E T$ InputsIRun 4 NASIFY 2003 Run 4 NAS.mdb

Scenario Last Executed: $12 / 14 / 01 \quad 1: 24: 50$ PM 
Consumer Cost Savings (million \$/yr)

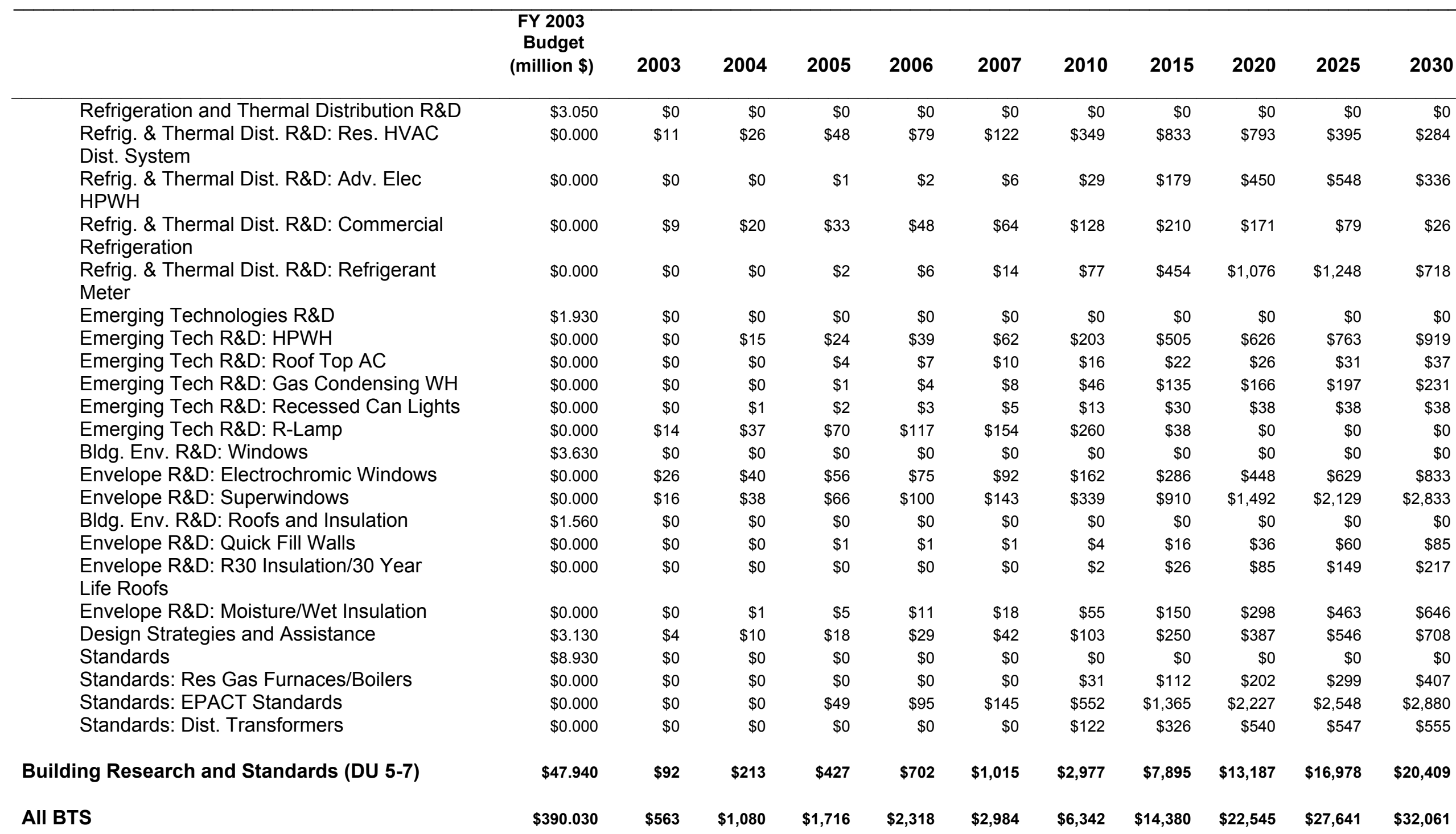

Run Title: FY 2003 Run 4 NAS

Scenario Last Executed: $12 / 14 / 01 \quad 1: 24: 50$ PM

MDB: $\quad$ K:IMETRICSIFY2003IBESET InputsIRun 4 NASIFY 2003 Run 4 NAS.mdb 


\section{Site Electricity Savings}


Site Electricity Energy Savings (TBtu/yr)

\begin{tabular}{|c|c|c|c|c|c|c|c|c|c|c|c|c|}
\hline & & $\begin{array}{c}\text { FY } 2003 \\
\text { Budget } \\
\text { (million \$) }\end{array}$ & 2003 & 2004 & 2005 & 2006 & 2007 & 2010 & 2015 & 2020 & 2025 & 2030 \\
\hline \multirow[t]{2}{*}{1} & State Energy Program & $\$ 38.800$ & 0.8 & 1.7 & 2.5 & 3.4 & 4.2 & 6.8 & 11.0 & 12.7 & 12.7 & 12.7 \\
\hline & State Formula Grants & $\$ 38.800$ & 0.8 & 1.7 & 2.5 & 3.4 & 4.2 & 6.8 & 11.0 & 12.7 & 12.7 & 12.7 \\
\hline \multirow[t]{2}{*}{2} & Weatherization Assistance Program & $\$ 277.100$ & 0.7 & 1.4 & 2.0 & 2.7 & 3.4 & 5.4 & 8.9 & 10.4 & 10.4 & 10.4 \\
\hline & Weatherization Assistance & $\$ 277.100$ & 0.7 & 1.4 & 2.0 & 2.7 & 3.4 & 5.4 & 8.9 & 10.4 & 10.4 & 10.4 \\
\hline \multirow[t]{4}{*}{3} & Community Energy Program & $\$ 19.990$ & 11.0 & 20.3 & 30.2 & 35.1 & 40.3 & 60.1 & 95.9 & 122.6 & 145.9 & 167.5 \\
\hline & Rebuild America & $\$ 12.720$ & 0.1 & 0.5 & 0.9 & 1.5 & 2.0 & 4.0 & 5.2 & 5.2 & 5.5 & 5.6 \\
\hline & Training and Technical Assistance & $\$ 4.860$ & 5.3 & 8.7 & 12.6 & 16.9 & 21.6 & 39.4 & 74.0 & 100.7 & 123.7 & 145.2 \\
\hline & Information Outreach & $\$ 2.410$ & 5.6 & 11.1 & 16.7 & 16.7 & 16.7 & 16.7 & 16.7 & 16.7 & 16.7 & 16.7 \\
\hline \multirow[t]{8}{*}{4} & Energy Star Program & $\$ 6.200$ & 3.5 & 6.4 & 11.0 & 17.1 & 25.0 & 58.1 & 144.5 & 234.1 & 261.7 & 275.5 \\
\hline & Energy Star & $\$ 6.200$ & 0.0 & 0.0 & 0.0 & 0.0 & 0.0 & 0.0 & 0.0 & 0.0 & 0.0 & 0.0 \\
\hline & Energy Star: Clothes Washers & $\$ 0.000$ & 2.0 & 2.6 & 3.4 & 4.2 & 5.1 & 7.7 & 10.8 & 13.5 & 16.4 & 19.7 \\
\hline & Energy Star: Electric Water Heaters & $\$ 0.000$ & 0.0 & 0.0 & 0.2 & 0.4 & 0.8 & 4.9 & 13.6 & 24.0 & 36.0 & 49.8 \\
\hline & Energy Star: Gas Water Heaters & $\$ 0.000$ & 0.0 & 0.0 & 0.0 & 0.0 & 0.0 & 0.0 & 0.0 & 0.0 & 0.0 & 0.0 \\
\hline & Energy Star: Room Air Cond & $\$ 0.000$ & 0.1 & 0.1 & 0.2 & 0.3 & 0.3 & 0.6 & 1.0 & 1.4 & 1.8 & 2.3 \\
\hline & Energy Star: CFLs & $\$ 0.000$ & 1.3 & 3.6 & 6.9 & 11.4 & 17.6 & 42.3 & 114.1 & 188.1 & 198.0 & 191.6 \\
\hline & Energy Star: Dishwashers & $\$ 0.000$ & 0.0 & 0.1 & 0.1 & 0.2 & 0.3 & 0.5 & 1.0 & 1.5 & 2.1 & 2.8 \\
\hline \multicolumn{2}{|r|}{ Building Technology Assistance (DU 1-4) } & $\$ 342.090$ & 16.0 & 29.8 & 45.7 & 58.3 & 72.9 & 130.4 & 260.2 & 379.7 & 430.7 & 466.1 \\
\hline \multirow[t]{3}{*}{5} & Residential Buildings Integration & $\$ 13.430$ & 0.0 & 0.1 & 0.1 & 0.3 & 0.5 & 1.9 & 6.5 & 11.6 & 16.7 & 21.9 \\
\hline & Res. Technology Research \& Development & $\$ 12.840$ & 0.0 & 0.1 & 0.1 & 0.3 & 0.5 & 1.4 & 3.1 & 4.0 & 4.5 & 4.9 \\
\hline & Residential Building Codes & $\$ 0.590$ & 0.0 & 0.0 & 0.0 & 0.0 & 0.0 & 0.5 & 3.4 & 7.6 & 12.2 & 17.0 \\
\hline \multirow[t]{3}{*}{6} & Commercial Buildings Integration & $\$ 4.990$ & 0.2 & 0.4 & 0.8 & 1.6 & 2.4 & 11.1 & 46.5 & 81.2 & 117.4 & 155.0 \\
\hline & Com. Technology Development & $\$ 4.450$ & 0.2 & 0.4 & 0.8 & 1.5 & 2.2 & 7.7 & 25.8 & 37.9 & 48.7 & 58.9 \\
\hline & Commercial Building Codes & $\$ 0.540$ & 0.0 & 0.0 & 0.0 & 0.1 & 0.2 & 3.4 & 20.7 & 43.3 & 68.7 & 96.1 \\
\hline \multirow[t]{5}{*}{7} & Building Equipment and Materials & $\$ 29.520$ & 3.3 & 7.5 & 15.7 & 25.7 & 37.3 & 107.0 & 261.9 & 444.9 & 556.9 & 638.2 \\
\hline & Lighting R\&D & $\$ 7.290$ & 0.0 & 0.0 & 0.0 & 0.0 & 0.0 & 0.0 & 0.0 & 0.0 & 0.0 & 0.0 \\
\hline & Lighting R\&D: Two-Photon Phosphors & $\$ 0.000$ & 0.0 & 0.0 & 0.0 & 0.0 & 0.0 & 1.3 & 19.7 & 57.3 & 97.4 & 140.2 \\
\hline & Lighting R\&D: Solid State Lighting & $\$ 0.000$ & 0.0 & 0.0 & 0.0 & 0.0 & 0.0 & 0.1 & 5.1 & 21.0 & 38.1 & 56.7 \\
\hline & Lighting R\&D: Controls & $\$ 0.000$ & 0.2 & 0.4 & 0.8 & 1.3 & 2.1 & 5.6 & 12.7 & 17.2 & 21.8 & 25.9 \\
\hline
\end{tabular}

Run Title: FY 2003 Run 4 NAS

MDB: $\quad K: I M E T R I C S I F Y 2003 \mid B E S E T$ InputsIRun 4 NASIFY 2003 Run 4 NAS.mdb

Scenario Last Executed: $12 / 14 / 01 \quad 1: 24: 50$ PM 
Site Electricity Energy Savings (TBtu/yr)

\begin{tabular}{|c|c|c|c|c|c|c|c|c|c|c|c|}
\hline & $\begin{array}{c}\text { FY } 2003 \\
\text { Budget } \\
\text { (million \$) }\end{array}$ & 2003 & 2004 & 2005 & 2006 & 2007 & 2010 & 2015 & 2020 & 2025 & 2030 \\
\hline Refrigeration and Thermal Distribution R\&D & $\$ 3.050$ & 0.0 & 0.0 & 0.0 & 0.0 & 0.0 & 0.0 & 0.0 & 0.0 & 0.0 & 0.0 \\
\hline $\begin{array}{l}\text { Refrig. \& Thermal Dist. R\&D: Res. HVAC } \\
\text { Dist. System }\end{array}$ & $\$ 0.000$ & 0.2 & 0.6 & 1.0 & 1.7 & 2.6 & 7.6 & 18.2 & 17.5 & 9.2 & 7.2 \\
\hline $\begin{array}{l}\text { Refrig. \& Thermal Dist. R\&D: Adv. Elec } \\
\text { HPWH }\end{array}$ & $\$ 0.000$ & 0.0 & 0.0 & 0.0 & 0.1 & 0.3 & 1.3 & 8.3 & 20.6 & 24.9 & 15.1 \\
\hline $\begin{array}{l}\text { Refrig. \& Thermal Dist. R\&D: Refrigerant } \\
\text { Meter }\end{array}$ & $\$ 0.000$ & 0.0 & 0.0 & 0.1 & 0.3 & 0.7 & 3.7 & 21.6 & 50.5 & 58.0 & 33.0 \\
\hline Emerging Technologies R\&D & $\$ 1.930$ & 0.0 & 0.0 & 0.0 & 0.0 & 0.0 & 0.0 & 0.0 & 0.0 & 0.0 & 0.0 \\
\hline Emerging Tech R\&D: HPWH & $\$ 0.000$ & 0.0 & 0.7 & 1.1 & 1.8 & 2.8 & 9.3 & 22.9 & 28.2 & 34.2 & 40.9 \\
\hline Emerging Tech R\&D: Roof Top AC & $\$ 0.000$ & 0.0 & 0.0 & 0.2 & 0.4 & 0.5 & 0.9 & 1.2 & 1.5 & 1.7 & 2.0 \\
\hline Emerging Tech R\&D: Gas Condensing WH & $\$ 0.000$ & 0.0 & 0.0 & 0.0 & 0.0 & 0.0 & 0.0 & 0.0 & 0.0 & 0.0 & 0.0 \\
\hline Emerging Tech R\&D: Recessed Can Lights & $\$ 0.000$ & 0.0 & 0.0 & 0.1 & 0.1 & 0.2 & 0.6 & 1.4 & 1.7 & 1.7 & 1.7 \\
\hline Bldg. Env. R\&D: Windows & $\$ 3.630$ & 0.0 & 0.0 & 0.0 & 0.0 & 0.0 & 0.0 & 0.0 & 0.0 & 0.0 & 0.0 \\
\hline Envelope R\&D: Electrochromic Windows & $\$ 0.000$ & 1.5 & 2.2 & 3.2 & 4.4 & 5.6 & 10.1 & 17.2 & 25.8 & 35.3 & 45.8 \\
\hline Envelope R\&D: Superwindows & $\$ 0.000$ & 0.2 & 0.5 & 1.0 & 1.5 & 2.2 & 5.4 & 15.2 & 25.9 & 38.1 & 52.2 \\
\hline Bldg. Env. R\&D: Roofs and Insulation & $\$ 1.560$ & 0.0 & 0.0 & 0.0 & 0.0 & 0.0 & 0.0 & 0.0 & 0.0 & 0.0 & 0.0 \\
\hline Envelope R\&D: Quick Fill Walls & $\$ 0.000$ & 0.0 & 0.0 & 0.0 & 0.0 & 0.0 & 0.0 & 0.2 & 0.4 & 0.7 & 1.0 \\
\hline $\begin{array}{l}\text { Envelope R\&D: R30 Insulation/30 Year } \\
\text { Life Roofs }\end{array}$ & $\$ 0.000$ & 0.0 & 0.0 & 0.0 & 0.0 & 0.0 & 0.0 & 0.3 & 0.7 & 1.2 & 1.7 \\
\hline Envelope R\&D: Moisture/Wet Insulation & $\$ 0.000$ & 0.0 & 0.0 & 0.0 & 0.0 & 0.0 & 0.2 & 1.0 & 2.4 & 4.1 & 6.0 \\
\hline Design Strategies and Assistance & $\$ 3.130$ & 0.2 & 0.4 & 0.8 & 1.2 & 1.8 & 4.6 & 11.1 & 16.9 & 23.5 & 30.0 \\
\hline Standards & $\$ 8.930$ & 0.0 & 0.0 & 0.0 & 0.0 & 0.0 & 0.0 & 0.0 & 0.0 & 0.0 & 0.0 \\
\hline Standards: Res Gas Furnaces/Boilers & $\$ 0.000$ & 0.0 & 0.0 & 0.0 & 0.0 & 0.0 & 0.0 & 0.0 & -0.1 & -0.1 & -0.1 \\
\hline Standards: EPACT Standards & $\$ 0.000$ & 0.0 & 0.0 & 2.5 & 5.0 & 7.9 & 30.4 & 74.6 & 118.8 & 133.3 & 147.8 \\
\hline Standards: Dist. Transformers & $\$ 0.000$ & 0.0 & 0.0 & 0.0 & 0.0 & 0.0 & 6.9 & 18.4 & 29.8 & 29.8 & 29.8 \\
\hline Building Research and Standards (DU 5-7) & $\$ 47.940$ & 3.5 & 8.0 & 16.6 & 27.6 & 40.2 & 120.0 & 315.0 & 537.7 & 690.9 & 815.1 \\
\hline All BTS & $\$ 390.030$ & 19.5 & 37.8 & 62.4 & 85.9 & 113.1 & 250.4 & 575.2 & 917.4 & $1,121.6$ & $1,281.2$ \\
\hline
\end{tabular}

Run Title: FY 2003 Run 4 NAS

Scenario Last Executed: $12 / 14 / 01 \quad 1: 24: 50$ PM

MDB: $\quad$ K:IMETRICSIFY2003IBESET InputsIRun 4 NASIFY 2003 Run 4 NAS.mdb 


\section{Site Oil Savings}


Direct Fuel Oil Energy Savings (TBtu/yr)

\begin{tabular}{|c|c|c|c|c|c|c|c|c|c|c|c|c|}
\hline & & $\begin{array}{c}\text { FY } 2003 \\
\text { Budget } \\
\text { (million \$) }\end{array}$ & 2003 & 2004 & 2005 & 2006 & 2007 & 2010 & 2015 & 2020 & 2025 & 2030 \\
\hline \multirow[t]{2}{*}{1} & State Energy Program & $\$ 38.800$ & 1.0 & 2.0 & 3.0 & 3.9 & 4.9 & 7.9 & 12.8 & 14.8 & 14.8 & 14.8 \\
\hline & State Formula Grants & $\$ 38.800$ & 1.0 & 2.0 & 3.0 & 3.9 & 4.9 & 7.9 & 12.8 & 14.8 & 14.8 & 14.8 \\
\hline \multirow[t]{2}{*}{2} & Weatherization Assistance Program & $\$ 277.100$ & 1.9 & 3.8 & 5.6 & 7.5 & 9.4 & 15.2 & 25.0 & 29.1 & 29.3 & 29.3 \\
\hline & Weatherization Assistance & $\$ 277.100$ & 1.9 & 3.8 & 5.6 & 7.5 & 9.4 & 15.2 & 25.0 & 29.1 & 29.3 & 29.3 \\
\hline \multirow[t]{4}{*}{3} & Community Energy Program & $\$ 19.990$ & 0.9 & 1.7 & 2.6 & 2.9 & 3.1 & 4.2 & 6.4 & 7.9 & 9.2 & 10.3 \\
\hline & Rebuild America & $\$ 12.720$ & 0.0 & 0.1 & 0.2 & 0.3 & 0.4 & 0.8 & 1.0 & 0.9 & 0.9 & 0.9 \\
\hline & Training and Technical Assistance & $\$ 4.860$ & 0.2 & 0.2 & 0.3 & 0.5 & 0.6 & 1.3 & 3.3 & 4.9 & 6.2 & 7.4 \\
\hline & Information Outreach & $\$ 2.410$ & 0.7 & 1.4 & 2.1 & 2.1 & 2.1 & 2.1 & 2.1 & 2.1 & 2.1 & 2.1 \\
\hline \multirow[t]{8}{*}{4} & Energy Star Program & $\$ 6.200$ & 0.6 & 0.8 & 1.0 & 1.3 & 1.5 & 2.2 & 2.8 & 3.3 & 3.8 & 4.4 \\
\hline & Energy Star & $\$ 6.200$ & 0.0 & 0.0 & 0.0 & 0.0 & 0.0 & 0.0 & 0.0 & 0.0 & 0.0 & 0.0 \\
\hline & Energy Star: Clothes Washers & $\$ 0.000$ & 0.6 & 0.8 & 1.1 & 1.3 & 1.6 & 2.3 & 3.1 & 3.8 & 4.4 & 5.0 \\
\hline & Energy Star: Electric Water Heaters & $\$ 0.000$ & 0.0 & 0.0 & 0.0 & 0.0 & 0.0 & 0.0 & 0.0 & 0.0 & 0.0 & 0.0 \\
\hline & Energy Star: Gas Water Heaters & $\$ 0.000$ & 0.0 & 0.0 & 0.0 & 0.0 & 0.0 & 0.0 & 0.0 & 0.0 & 0.0 & 0.0 \\
\hline & Energy Star: Room Air Cond & $\$ 0.000$ & 0.0 & 0.0 & 0.0 & 0.0 & 0.0 & 0.0 & 0.0 & 0.0 & 0.0 & 0.0 \\
\hline & Energy Star: CFLs & $\$ 0.000$ & 0.0 & 0.0 & 0.0 & 0.0 & 0.0 & -0.1 & -0.3 & -0.5 & -0.6 & -0.5 \\
\hline & Energy Star: Dishwashers & $\$ 0.000$ & 0.0 & 0.0 & 0.0 & 0.0 & 0.0 & 0.0 & 0.0 & 0.0 & 0.0 & 0.0 \\
\hline \multicolumn{2}{|r|}{ Building Technology Assistance (DU 1-4) } & $\$ 342.090$ & 4.4 & 8.2 & 12.2 & 15.6 & 19.0 & 29.5 & 46.9 & 55.0 & 57.1 & 58.8 \\
\hline \multirow[t]{3}{*}{5} & Residential Buildings Integration & $\$ 13.430$ & 0.0 & 0.0 & 0.0 & 0.0 & 0.1 & 0.5 & 3.0 & 6.2 & 9.7 & 13.2 \\
\hline & Res. Technology Research \& Development & $\$ 12.840$ & 0.0 & 0.0 & 0.0 & 0.0 & 0.1 & 0.2 & 0.5 & 0.6 & 0.7 & 0.7 \\
\hline & Residential Building Codes & $\$ 0.590$ & 0.0 & 0.0 & 0.0 & 0.0 & 0.0 & 0.3 & 2.5 & 5.6 & 9.0 & 12.5 \\
\hline \multirow[t]{3}{*}{6} & Commercial Buildings Integration & $\$ 4.990$ & 0.0 & 0.1 & 0.2 & 0.3 & 0.4 & 1.3 & 4.0 & 5.0 & 5.4 & 5.7 \\
\hline & Com. Technology Development & $\$ 4.450$ & 0.0 & 0.1 & 0.2 & 0.3 & 0.4 & 1.3 & 4.0 & 5.0 & 5.4 & 5.7 \\
\hline & Commercial Building Codes & $\$ 0.540$ & 0.0 & 0.0 & 0.0 & 0.0 & 0.0 & 0.0 & 0.0 & 0.0 & 0.0 & 0.0 \\
\hline \multirow{4}{*}{7} & Lighting R\&D: Two-Photon Phosphors & $\$ 0.000$ & 0.0 & 0.0 & 0.0 & 0.0 & 0.0 & 0.0 & 0.0 & 0.0 & 0.0 & 0.0 \\
\hline & Lighting R\&D: Solid State Lighting & $\$ 0.000$ & 0.0 & 0.0 & 0.0 & 0.0 & 0.0 & 0.0 & 0.0 & 0.0 & 0.0 & 0.0 \\
\hline & Lighting R\&D: Controls & $\$ 0.000$ & 0.0 & 0.0 & 0.0 & 0.0 & 0.0 & 0.0 & 0.0 & 0.0 & 0.0 & 0.0 \\
\hline & Refrigeration and Thermal Distribution R\&D & $\$ 3.050$ & 0.0 & 0.0 & 0.0 & 0.0 & 0.0 & 0.0 & 0.0 & 0.0 & 0.0 & 0.0 \\
\hline
\end{tabular}

Run Title: FY 2003 Run 4 NAS

Scenario Last Executed: 12/14/01 1:24:50 PM

MDB: $\quad$ K:IMETRICSIFY2003IBESET InputsIRun 4 NASIFY 2003 Run 4 NAS.mdb 
Direct Fuel Oil Energy Savings (TBtu/yr)

\begin{tabular}{|c|c|c|c|c|c|c|c|c|c|c|c|}
\hline & $\begin{array}{c}\text { FY } 2003 \\
\text { Budget } \\
\text { (million \$) }\end{array}$ & 2003 & 2004 & 2005 & 2006 & 2007 & 2010 & 2015 & 2020 & 2025 & 2030 \\
\hline $\begin{array}{l}\text { Refrig. \& Thermal Dist. R\&D: Res. HVAC } \\
\text { Dist. System }\end{array}$ & $\$ 0.000$ & 0.0 & 0.1 & 0.1 & 0.2 & 0.4 & 1.1 & 2.5 & 2.4 & 1.2 & 0.8 \\
\hline $\begin{array}{l}\text { Refrig. \& Thermal Dist. R\&D: Adv. Elec } \\
\text { HPWH }\end{array}$ & $\$ 0.000$ & 0.0 & 0.0 & 0.0 & 0.0 & 0.0 & 0.0 & 0.0 & 0.0 & 0.0 & 0.0 \\
\hline $\begin{array}{l}\text { Refrig. \& Thermal Dist. R\&D: Commercial } \\
\text { Refrigeration }\end{array}$ & $\$ 0.000$ & 0.0 & 0.0 & 0.0 & 0.0 & 0.0 & 0.0 & 0.0 & 0.0 & 0.0 & 0.0 \\
\hline $\begin{array}{l}\text { Refrig. \& Thermal Dist. R\&D: Refrigerant } \\
\text { Meter }\end{array}$ & $\$ 0.000$ & 0.0 & 0.0 & 0.0 & 0.0 & 0.0 & 0.0 & 0.0 & 0.0 & 0.0 & 0.0 \\
\hline Emerging Technologies R\&D & $\$ 1.930$ & 0.0 & 0.0 & 0.0 & 0.0 & 0.0 & 0.0 & 0.0 & 0.0 & 0.0 & 0.0 \\
\hline Emerging Tech R\&D: HPWH & $\$ 0.000$ & 0.0 & 0.0 & 0.0 & 0.0 & 0.0 & 0.0 & 0.0 & 0.0 & 0.0 & 0.0 \\
\hline Emerging Tech R\&D: Roof Top AC & $\$ 0.000$ & 0.0 & 0.0 & 0.0 & 0.0 & 0.0 & 0.0 & 0.0 & 0.0 & 0.0 & 0.0 \\
\hline Emerging Tech R\&D: Gas Condensing WH & $\$ 0.000$ & 0.0 & 0.0 & 0.0 & 0.0 & 0.0 & 0.0 & 0.0 & 0.0 & 0.0 & 0.0 \\
\hline Emerging Tech R\&D: Recessed Can Lights & $\$ 0.000$ & 0.0 & 0.0 & 0.0 & 0.0 & 0.0 & 0.0 & 0.0 & 0.0 & 0.0 & 0.0 \\
\hline Emerging Tech R\&D: R-Lamp & $\$ 0.000$ & 0.0 & 0.0 & 0.0 & 0.0 & 0.0 & 0.0 & 0.0 & 0.0 & 0.0 & 0.0 \\
\hline Envelope R\&D: Electrochromic Windows & $\$ 0.000$ & 0.0 & 0.0 & 0.0 & 0.0 & -0.1 & -0.1 & -0.2 & -0.3 & -0.4 & -0.5 \\
\hline Envelope R\&D: Superwindows & $\$ 0.000$ & 0.5 & 1.1 & 1.8 & 2.7 & 3.8 & 8.2 & 19.2 & 26.6 & 33.5 & 39.9 \\
\hline Bldg. Env. R\&D: Roofs and Insulation & $\$ 1.560$ & 0.0 & 0.0 & 0.0 & 0.0 & 0.0 & 0.0 & 0.0 & 0.0 & 0.0 & 0.0 \\
\hline Envelope R\&D: Quick Fill Walls & $\$ 0.000$ & 0.0 & 0.0 & 0.0 & 0.0 & 0.0 & 0.0 & 0.1 & 0.2 & 0.3 & 0.4 \\
\hline $\begin{array}{l}\text { Envelope R\&D: R30 Insulation/30 Year } \\
\text { Life Roofs }\end{array}$ & $\$ 0.000$ & 0.0 & 0.0 & 0.0 & 0.0 & 0.0 & 0.0 & 0.4 & 1.1 & 1.8 & 2.5 \\
\hline Envelope R\&D: Moisture/Wet Insulation & $\$ 0.000$ & 0.0 & 0.0 & 0.0 & 0.0 & 0.0 & 0.1 & 0.4 & 1.1 & 1.7 & 2.4 \\
\hline Design Strategies and Assistance & $\$ 3.130$ & 0.0 & 0.0 & 0.1 & 0.1 & 0.1 & 0.3 & 0.8 & 1.2 & 1.7 & 2.2 \\
\hline Standards & $\$ 8.930$ & 0.0 & 0.0 & 0.0 & 0.0 & 0.0 & 0.0 & 0.0 & 0.0 & 0.0 & 0.0 \\
\hline Standards: Res Gas Furnaces/Boilers & $\$ 0.000$ & 0.0 & 0.0 & 0.0 & 0.0 & 0.0 & 0.0 & 0.0 & 0.0 & 0.0 & 0.0 \\
\hline Standards: EPACT Standards & $\$ 0.000$ & 0.0 & 0.0 & 0.0 & 0.0 & 0.0 & 0.0 & 0.0 & 0.0 & 0.0 & 0.0 \\
\hline Standards: Dist. Transformers & $\$ 0.000$ & 0.0 & 0.0 & 0.0 & 0.0 & 0.0 & 0.0 & 0.0 & 0.0 & 0.0 & 0.0 \\
\hline Building Research and Standards (DU 5-7) & $\$ 47.940$ & 0.6 & 1.2 & 2.2 & 3.3 & 4.7 & 11.5 & 30.2 & 43.5 & 54.9 & 66.6 \\
\hline All BTS & $\$ 390.030$ & 5.0 & 9.5 & 14.4 & 19.0 & 23.7 & 40.9 & 77.1 & 98.5 & 111.9 & 125.4 \\
\hline
\end{tabular}

Run Title: FY 2003 Run 4 NAS

Scenario Last Executed: $12 / 14 / 01 \quad 1: 24: 50$ PM

MDB: $\quad$ K:IMETRICSIFY2003IBESET InputsIRun 4 NASIFY 2003 Run 4 NAS.mdb 


\section{Site Natural Gas Savings}


Direct Natural Gas Energy Savings (TBtu/yr)

\begin{tabular}{|c|c|c|c|c|c|c|c|c|c|c|c|c|}
\hline & & $\begin{array}{c}\text { FY } 2003 \\
\text { Budget } \\
\text { (million \$) }\end{array}$ & 2003 & 2004 & 2005 & 2006 & 2007 & 2010 & 2015 & 2020 & 2025 & 2030 \\
\hline \multirow[t]{2}{*}{1} & State Energy Program & $\$ 38.800$ & 0.3 & 0.5 & 0.7 & 1.0 & 1.2 & 2.0 & 3.2 & 3.7 & 3.7 & 3.7 \\
\hline & State Formula Grants & $\$ 38.800$ & 0.3 & 0.5 & 0.7 & 1.0 & 1.2 & 2.0 & 3.2 & 3.7 & 3.7 & 3.7 \\
\hline \multirow[t]{2}{*}{2} & Weatherization Assistance Program & $\$ 277.100$ & 4.5 & 9.0 & 13.5 & 18.0 & 22.5 & 36.3 & 59.6 & 69.5 & 70.1 & 70.1 \\
\hline & Weatherization Assistance & $\$ 277.100$ & 4.5 & 9.0 & 13.5 & 18.0 & 22.5 & 36.3 & 59.6 & 69.5 & 70.1 & 70.1 \\
\hline \multirow[t]{4}{*}{3} & Community Energy Program & $\$ 19.990$ & 8.5 & 17.3 & 26.2 & 28.4 & 30.2 & 38.7 & 52.5 & 61.8 & 70.4 & 77.9 \\
\hline & Rebuild America & $\$ 12.720$ & 0.3 & 1.3 & 2.3 & 4.0 & 5.3 & 10.4 & 13.1 & 12.8 & 13.1 & 13.2 \\
\hline & Training and Technical Assistance & $\$ 4.860$ & 0.5 & 0.7 & 0.9 & 1.4 & 2.0 & 5.4 & 16.4 & 26.0 & 34.3 & 41.7 \\
\hline & Information Outreach & $\$ 2.410$ & 7.7 & 15.3 & 23.0 & 23.0 & 23.0 & 23.0 & 23.0 & 23.0 & 23.0 & 23.0 \\
\hline \multirow[t]{9}{*}{4} & Energy Star Program & $\$ 6.200$ & 3.6 & 4.4 & 5.9 & 7.4 & 8.9 & 13.3 & 17.0 & 24.1 & 37.4 & 53.2 \\
\hline & Energy Star & $\$ 6.200$ & 0.0 & 0.0 & 0.0 & 0.0 & 0.0 & 0.0 & 0.0 & 0.0 & 0.0 & 0.0 \\
\hline & Energy Star: Clothes Washers & $\$ 0.000$ & 3.7 & 4.7 & 6.4 & 8.2 & 9.9 & 15.3 & 22.1 & 29.0 & 36.3 & 44.2 \\
\hline & Energy Star: Refrigerators & $\$ 0.000$ & 0.0 & 0.0 & 0.0 & 0.0 & 0.0 & 0.0 & 0.0 & 0.0 & 0.0 & 0.0 \\
\hline & Energy Star: Electric Water Heaters & $\$ 0.000$ & 0.0 & 0.0 & 0.0 & 0.0 & 0.0 & 0.0 & 0.0 & 0.0 & 0.0 & 0.0 \\
\hline & Energy Star: Gas Water Heaters & $\$ 0.000$ & 0.0 & 0.0 & 0.1 & 0.2 & 0.4 & 1.5 & 4.3 & 10.5 & 17.3 & 24.7 \\
\hline & Energy Star: Room Air Cond & $\$ 0.000$ & 0.0 & 0.0 & 0.0 & 0.0 & 0.0 & 0.0 & 0.0 & 0.0 & 0.0 & 0.0 \\
\hline & Energy Star: CFLs & $\$ 0.000$ & -0.1 & -0.3 & -0.6 & -0.9 & -1.5 & -3.5 & -9.4 & -15.4 & -16.2 & -15.7 \\
\hline & Energy Star: Dishwashers & $\$ 0.000$ & 0.0 & 0.0 & 0.0 & 0.0 & 0.0 & 0.0 & 0.0 & 0.0 & 0.0 & 0.0 \\
\hline \multicolumn{2}{|r|}{ Building Technology Assistance (DU 1-4) } & $\$ 342.090$ & 16.7 & 31.3 & 46.3 & 54.7 & 62.8 & 90.2 & 132.3 & 159.0 & 181.6 & 204.8 \\
\hline \multirow[t]{3}{*}{5} & Residential Buildings Integration & $\$ 13.430$ & 0.1 & 0.2 & 0.5 & 1.1 & 1.7 & 6.6 & 22.0 & 40.9 & 60.9 & 81.9 \\
\hline & Res. Technology Research \& Development & $\$ 12.840$ & 0.1 & 0.2 & 0.5 & 1.0 & 1.6 & 4.7 & 9.3 & 11.4 & 12.6 & 13.8 \\
\hline & Residential Building Codes & $\$ 0.590$ & 0.0 & 0.0 & 0.0 & 0.1 & 0.1 & 1.9 & 12.7 & 29.5 & 48.3 & 68.1 \\
\hline \multirow[t]{3}{*}{6} & Commercial Buildings Integration & $\$ 4.990$ & 0.3 & 0.6 & 1.3 & 2.2 & 3.2 & 10.9 & 34.4 & 46.2 & 54.4 & 61.7 \\
\hline & Com. Technology Development & $\$ 4.450$ & 0.3 & 0.6 & 1.3 & 2.2 & 3.2 & 10.9 & 34.4 & 46.1 & 54.3 & 61.5 \\
\hline & Commercial Building Codes & $\$ 0.540$ & 0.0 & 0.0 & 0.0 & 0.0 & 0.0 & 0.0 & 0.0 & 0.1 & 0.1 & 0.2 \\
\hline \multirow[t]{4}{*}{7} & Building Equipment and Materials & $\$ 29.520$ & 1.5 & 4.3 & 8.8 & 14.8 & 23.1 & 75.5 & 208.4 & 299.4 & 370.1 & 471.0 \\
\hline & Lighting R\&D & $\$ 7.290$ & 0.0 & 0.0 & 0.0 & 0.0 & 0.0 & 0.0 & 0.0 & 0.0 & 0.0 & 0.0 \\
\hline & Lighting R\&D: Two-Photon Phosphors & $\$ 0.000$ & 0.0 & 0.0 & 0.0 & 0.0 & 0.0 & 0.0 & 0.0 & 0.0 & 0.0 & 0.0 \\
\hline & Lighting R\&D: Solid State Lighting & $\$ 0.000$ & 0.0 & 0.0 & 0.0 & 0.0 & 0.0 & 0.0 & 0.0 & 0.0 & 0.0 & 0.0 \\
\hline
\end{tabular}

Run Title: FY 2003 Run 4 NAS

Scenario Last Executed: $12 / 14 / 01 \quad 1: 24: 50$ PM

MDB: $\quad$ K:IMETRICSIFY2003IBESET InputsIRun 4 NASIFY 2003 Run 4 NAS.mdb 
Direct Natural Gas Energy Savings (TBtu/yr)

\begin{tabular}{|c|c|c|c|c|c|c|c|c|c|c|c|}
\hline & $\begin{array}{c}\text { FY } 2003 \\
\text { Budget } \\
\text { (million \$) }\end{array}$ & 2003 & 2004 & 2005 & 2006 & 2007 & 2010 & 2015 & 2020 & 2025 & 2030 \\
\hline Lighting R\&D: Controls & $\$ 0.000$ & 0.0 & 0.0 & 0.0 & 0.0 & 0.0 & 0.0 & 0.0 & 0.0 & 0.0 & 0.0 \\
\hline Refrigeration and Thermal Distribution R\&D & $\$ 3.050$ & 0.0 & 0.0 & 0.0 & 0.0 & 0.0 & 0.0 & 0.0 & 0.0 & 0.0 & 0.0 \\
\hline $\begin{array}{l}\text { Refrig. \& Thermal Dist. R\&D: Res. HVAC } \\
\text { Dist. System }\end{array}$ & $\$ 0.000$ & 0.8 & 2.0 & 3.7 & 6.1 & 9.4 & 26.9 & 64.0 & 59.1 & 27.4 & 17.6 \\
\hline $\begin{array}{l}\text { Refrig. \& Thermal Dist. R\&D: Adv. Elec } \\
\text { HPWH }\end{array}$ & $\$ 0.000$ & 0.0 & 0.0 & 0.0 & 0.0 & 0.0 & 0.0 & 0.0 & 0.0 & 0.0 & 0.0 \\
\hline $\begin{array}{l}\text { Refrig. \& Thermal Dist. R\&D: Commercial } \\
\text { Refrigeration }\end{array}$ & $\$ 0.000$ & 0.1 & 0.2 & 0.3 & 0.5 & 0.6 & 1.3 & 2.1 & 1.7 & 0.8 & 0.2 \\
\hline $\begin{array}{l}\text { Refrig. \& Thermal Dist. R\&D: Refrigerant } \\
\text { Meter }\end{array}$ & $\$ 0.000$ & 0.0 & 0.0 & 0.0 & 0.0 & 0.0 & 0.0 & 0.0 & 0.0 & 0.0 & 0.0 \\
\hline Emerging Technologies R\&D & $\$ 1.930$ & 0.0 & 0.0 & 0.0 & 0.0 & 0.0 & 0.0 & 0.0 & 0.0 & 0.0 & 0.0 \\
\hline Emerging Tech R\&D: HPWH & $\$ 0.000$ & 0.0 & 0.0 & 0.0 & 0.0 & 0.0 & 0.0 & 0.0 & 0.0 & 0.0 & 0.0 \\
\hline Emerging Tech R\&D: Roof Top AC & $\$ 0.000$ & 0.0 & 0.0 & 0.0 & 0.0 & 0.0 & 0.0 & 0.0 & 0.0 & 0.0 & 0.0 \\
\hline Emerging Tech R\&D: Gas Condensing WH & $\$ 0.000$ & 0.0 & 0.0 & 0.2 & 0.6 & 1.2 & 7.0 & 21.0 & 25.3 & 30.1 & 35.2 \\
\hline Emerging Tech R\&D: R-Lamp & $\$ 0.000$ & -0.1 & -0.1 & -0.3 & -0.5 & -0.6 & -1.0 & -0.1 & 0.0 & 0.0 & 0.0 \\
\hline Bldg. Env. R\&D: Windows & $\$ 3.630$ & 0.0 & 0.0 & 0.0 & 0.0 & 0.0 & 0.0 & 0.0 & 0.0 & 0.0 & 0.0 \\
\hline Envelope R\&D: Electrochromic Windows & $\$ 0.000$ & -0.7 & -1.0 & -1.3 & -1.6 & -2.0 & -2.7 & -3.2 & -3.1 & -3.0 & -2.8 \\
\hline Envelope R\&D: Superwindows & $\$ 0.000$ & 1.2 & 2.8 & 4.8 & 7.2 & 10.3 & 24.5 & 66.2 & 107.9 & 152.8 & 201.2 \\
\hline Bldg. Env. R\&D: Roofs and Insulation & $\$ 1.560$ & 0.0 & 0.0 & 0.0 & 0.0 & 0.0 & 0.0 & 0.0 & 0.0 & 0.0 & 0.0 \\
\hline Envelope R\&D: Quick Fill Walls & $\$ 0.000$ & 0.0 & 0.0 & 0.1 & 0.1 & 0.1 & 0.4 & 1.7 & 3.9 & 6.4 & 9.1 \\
\hline $\begin{array}{l}\text { Envelope R\&D: R30 Insulation/30 Year } \\
\text { Life Roofs }\end{array}$ & $\$ 0.000$ & 0.0 & 0.0 & 0.0 & 0.0 & 0.0 & 0.2 & 3.4 & 11.6 & 20.1 & 28.8 \\
\hline Envelope R\&D: Moisture/Wet Insulation & $\$ 0.000$ & 0.0 & 0.2 & 0.8 & 1.5 & 2.6 & 7.6 & 19.4 & 36.1 & 54.6 & 74.9 \\
\hline Design Strategies and Assistance & $\$ 3.130$ & 0.1 & 0.3 & 0.6 & 1.0 & 1.4 & 3.6 & 8.7 & 12.9 & 18.0 & 22.9 \\
\hline Standards & $\$ 8.930$ & 0.0 & 0.0 & 0.0 & 0.0 & 0.0 & 0.0 & 0.0 & 0.0 & 0.0 & 0.0 \\
\hline Standards: Res Gas Furnaces/Boilers & $\$ 0.000$ & 0.0 & 0.0 & 0.0 & 0.0 & 0.0 & 4.7 & 17.5 & 31.0 & 45.9 & 62.4 \\
\hline Standards: EPACT Standards & $\$ 0.000$ & 0.0 & 0.0 & 0.0 & 0.0 & 0.0 & 2.9 & 7.9 & 13.0 & 17.3 & 21.6 \\
\hline Standards: Dist. Transformers & $\$ 0.000$ & 0.0 & 0.0 & 0.0 & 0.0 & 0.0 & 0.0 & 0.0 & 0.0 & 0.0 & 0.0 \\
\hline Building Research and Standards (DU 5-7) & $\$ 47.940$ & 1.9 & 5.2 & 10.5 & 18.0 & 28.0 & 93.0 & 264.8 & 386.5 & 485.4 & 614.7 \\
\hline All BTS & $\$ 390.030$ & 18.6 & 36.4 & 56.8 & 72.7 & 90.9 & 183.2 & 397.0 & 545.6 & 667.0 & 819.5 \\
\hline
\end{tabular}

Run Title: FY 2003 Run 4 NAS

Scenario Last Executed: $12 / 14 / 01 \quad 1: 24: 50$ PM

MDB: $\quad$ K:IMETRICSIFY2003IBESET InputsIRun 4 NASIFY 2003 Run 4 NAS.mdb 


\section{Non-Energy Cost Savings}


Non-Energy Savings (million $\$ / y r)$

\begin{tabular}{|c|c|c|c|c|c|c|c|c|c|c|c|c|}
\hline & & $\begin{array}{c}\text { FY } 2003 \\
\text { Budget } \\
\text { (million \$) }\end{array}$ & 2003 & 2004 & 2005 & 2006 & 2007 & 2010 & 2015 & 2020 & 2025 & 2030 \\
\hline \multirow[t]{2}{*}{1} & State Energy Program & $\$ 38.800$ & $\$ 0$ & $\$ 0$ & $\$ 0$ & $\$ 0$ & $\$ 0$ & $\$ 0$ & $\$ 0$ & $\$ 0$ & $\$ 0$ & $\$ 0$ \\
\hline & State Formula Grants & $\$ 38.800$ & $\$ 0$ & $\$ 0$ & $\$ 0$ & $\$ 0$ & $\$ 0$ & $\$ 0$ & $\$ 0$ & $\$ 0$ & $\$ 0$ & $\$ 0$ \\
\hline \multirow[t]{2}{*}{2} & Weatherization Assistance Program & $\$ 277.100$ & $\$ 47$ & $\$ 47$ & $\$ 45$ & $\$ 45$ & $\$ 45$ & $\$ 46$ & $\$ 47$ & $\$ 47$ & $\$ 47$ & $\$ 47$ \\
\hline & Weatherization Assistance & $\$ 277.100$ & $\$ 47$ & $\$ 47$ & $\$ 45$ & $\$ 45$ & $\$ 45$ & $\$ 46$ & $\$ 47$ & $\$ 47$ & $\$ 47$ & $\$ 47$ \\
\hline \multirow[t]{4}{*}{3} & Community Energy Program & $\$ 19.990$ & $\$ 0$ & $\$ 0$ & $\$ 0$ & $\$ 0$ & $\$ 0$ & $\$ 0$ & $\$ 0$ & $\$ 0$ & $\$ 0$ & $\$ 0$ \\
\hline & Rebuild America & $\$ 12.720$ & $\$ 0$ & $\$ 0$ & $\$ 0$ & $\$ 0$ & $\$ 0$ & $\$ 0$ & $\$ 0$ & $\$ 0$ & $\$ 0$ & $\$ 0$ \\
\hline & Training and Technical Assistance & $\$ 4.860$ & $\$ 0$ & $\$ 0$ & $\$ 0$ & $\$ 0$ & $\$ 0$ & $\$ 0$ & $\$ 0$ & $\$ 0$ & $\$ 0$ & $\$ 0$ \\
\hline & Information Outreach & $\$ 2.410$ & $\$ 0$ & $\$ 0$ & $\$ 0$ & $\$ 0$ & $\$ 0$ & $\$ 0$ & $\$ 0$ & $\$ 0$ & $\$ 0$ & $\$ 0$ \\
\hline \multirow[t]{9}{*}{4} & Energy Star Program & $\$ 6.200$ & $\$ 0$ & $\$ 0$ & $\$ 0$ & $\$ 0$ & $\$ 0$ & $\$ 0$ & $\$ 0$ & $\$ 0$ & $\$ 0$ & $\$ 0$ \\
\hline & Energy Star & $\$ 6.200$ & $\$ 0$ & $\$ 0$ & $\$ 0$ & $\$ 0$ & $\$ 0$ & $\$ 0$ & $\$ 0$ & $\$ 0$ & $\$ 0$ & $\$ 0$ \\
\hline & Energy Star: Clothes Washers & $\$ 0.000$ & $\$ 0$ & $\$ 0$ & $\$ 0$ & $\$ 0$ & $\$ 0$ & $\$ 0$ & $\$ 0$ & $\$ 0$ & $\$ 0$ & $\$ 0$ \\
\hline & Energy Star: Refrigerators & $\$ 0.000$ & $\$ 0$ & $\$ 0$ & $\$ 0$ & $\$ 0$ & $\$ 0$ & $\$ 0$ & $\$ 0$ & $\$ 0$ & $\$ 0$ & $\$ 0$ \\
\hline & Energy Star: Electric Water Heaters & $\$ 0.000$ & $\$ 0$ & $\$ 0$ & $\$ 0$ & $\$ 0$ & $\$ 0$ & $\$ 0$ & $\$ 0$ & $\$ 0$ & $\$ 0$ & $\$ 0$ \\
\hline & Energy Star: Gas Water Heaters & $\$ 0.000$ & $\$ 0$ & $\$ 0$ & $\$ 0$ & $\$ 0$ & $\$ 0$ & $\$ 0$ & $\$ 0$ & $\$ 0$ & $\$ 0$ & $\$ 0$ \\
\hline & Energy Star: Room Air Cond & $\$ 0.000$ & $\$ 0$ & $\$ 0$ & $\$ 0$ & $\$ 0$ & $\$ 0$ & $\$ 0$ & $\$ 0$ & $\$ 0$ & $\$ 0$ & $\$ 0$ \\
\hline & Energy Star: CFLs & $\$ 0.000$ & $\$ 0$ & $\$ 0$ & $\$ 0$ & $\$ 0$ & $\$ 0$ & $\$ 0$ & $\$ 0$ & $\$ 0$ & $\$ 0$ & $\$ 0$ \\
\hline & Energy Star: Dishwashers & $\$ 0.000$ & $\$ 0$ & $\$ 0$ & $\$ 0$ & $\$ 0$ & $\$ 0$ & $\$ 0$ & $\$ 0$ & $\$ 0$ & $\$ 0$ & $\$ 0$ \\
\hline \multicolumn{2}{|r|}{ Building Technology Assistance (DU 1-4) } & $\$ 342.090$ & $\$ 47$ & $\$ 47$ & $\$ 45$ & $\$ 45$ & $\$ 45$ & $\$ 46$ & $\$ 47$ & $\$ 47$ & $\$ 47$ & $\$ 47$ \\
\hline \multirow[t]{3}{*}{5} & Residential Buildings Integration & $\$ 13.430$ & $\$ 0$ & $\$ 0$ & $\$ 0$ & $\$ 0$ & $\$ 0$ & $\$ 0$ & $\$ 0$ & $\$ 0$ & $\$ 0$ & $\$ 0$ \\
\hline & Res. Technology Research \& Development & $\$ 12.840$ & $\$ 0$ & $\$ 0$ & $\$ 0$ & $\$ 0$ & $\$ 0$ & $\$ 0$ & $\$ 0$ & $\$ 0$ & $\$ 0$ & $\$ 0$ \\
\hline & Residential Building Codes & $\$ 0.590$ & $\$ 0$ & $\$ 0$ & $\$ 0$ & $\$ 0$ & $\$ 0$ & $\$ 0$ & $\$ 0$ & $\$ 0$ & $\$ 0$ & $\$ 0$ \\
\hline \multirow[t]{3}{*}{6} & Commercial Buildings Integration & $\$ 4.990$ & $\$ 0$ & $\$ 0$ & $\$ 0$ & $\$ 0$ & $\$ 0$ & $\$ 0$ & $\$ 0$ & $\$ 0$ & $\$ 0$ & $\$ 0$ \\
\hline & Com. Technology Development & $\$ 4.450$ & $\$ 0$ & $\$ 0$ & $\$ 0$ & $\$ 0$ & $\$ 0$ & $\$ 0$ & $\$ 0$ & $\$ 0$ & $\$ 0$ & $\$ 0$ \\
\hline & Commercial Building Codes & $\$ 0.540$ & $\$ 0$ & $\$ 0$ & $\$ 0$ & $\$ 0$ & $\$ 0$ & $\$ 0$ & $\$ 0$ & $\$ 0$ & $\$ 0$ & $\$ 0$ \\
\hline \multirow[t]{6}{*}{7} & Building Equipment and Materials & $\$ 29.520$ & $\$ 0$ & $\$ 0$ & $\$ 0$ & $\$ 0$ & $\$ 0$ & $\$ 0$ & $\$ 0$ & $\$ 0$ & $\$ 0$ & $\$ 0$ \\
\hline & Lighting R\&D & $\$ 7.290$ & $\$ 0$ & $\$ 0$ & $\$ 0$ & $\$ 0$ & $\$ 0$ & $\$ 0$ & $\$ 0$ & $\$ 0$ & $\$ 0$ & $\$ 0$ \\
\hline & Lighting R\&D: Two-Photon Phosphors & $\$ 0.000$ & $\$ 0$ & $\$ 0$ & $\$ 0$ & $\$ 0$ & $\$ 0$ & $\$ 0$ & $\$ 0$ & $\$ 0$ & $\$ 0$ & $\$ 0$ \\
\hline & Lighting R\&D: Solid State Lighting & $\$ 0.000$ & $\$ 0$ & $\$ 0$ & $\$ 0$ & $\$ 0$ & $\$ 0$ & $\$ 0$ & $\$ 0$ & $\$ 0$ & $\$ 0$ & $\$ 0$ \\
\hline & Lighting R\&D: Controls & $\$ 0.000$ & $\$ 0$ & $\$ 0$ & $\$ 0$ & $\$ 0$ & $\$ 0$ & $\$ 0$ & $\$ 0$ & $\$ 0$ & $\$ 0$ & $\$ 0$ \\
\hline & Refrigeration and Thermal Distribution R\&D & $\$ 3.050$ & $\$ 0$ & $\$ 0$ & $\$ 0$ & $\$ 0$ & $\$ 0$ & $\$ 0$ & $\$ 0$ & $\$ 0$ & $\$ 0$ & $\$ 0$ \\
\hline
\end{tabular}

Run Title: FY 2003 Run 4 NAS

Scenario Last Executed: 12/14/01

1:24:50 PM

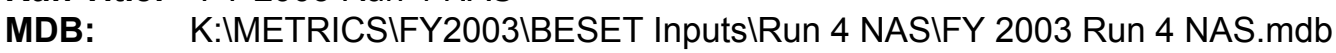


Non-Energy Savings (million $\$ / y r$ )

\begin{tabular}{|c|c|c|c|c|c|c|c|c|c|c|c|}
\hline & $\begin{array}{c}\text { FY } 2003 \\
\text { Budget } \\
\text { (million \$) }\end{array}$ & 2003 & 2004 & 2005 & 2006 & 2007 & 2010 & 2015 & 2020 & 2025 & 2030 \\
\hline $\begin{array}{l}\text { Refrig. \& Thermal Dist. R\&D: Res. HVAC } \\
\text { Dist. System }\end{array}$ & $\$ 0.000$ & $\$ 0$ & $\$ 0$ & $\$ 0$ & $\$ 0$ & $\$ 0$ & $\$ 0$ & $\$ 0$ & $\$ 0$ & $\$ 0$ & $\$ 0$ \\
\hline $\begin{array}{l}\text { Refrig. \& Thermal Dist. R\&D: Commercial } \\
\text { Refrigeration }\end{array}$ & $\$ 0.000$ & $\$ 0$ & $\$ 0$ & $\$ 0$ & $\$ 0$ & $\$ 0$ & $\$ 0$ & $\$ 0$ & $\$ 0$ & $\$ 0$ & $\$ 0$ \\
\hline $\begin{array}{l}\text { Refrig. \& Thermal Dist. R\&D: Refrigerant } \\
\text { Meter }\end{array}$ & $\$ 0.000$ & $\$ 0$ & $\$ 0$ & $\$ 0$ & $\$ 0$ & $\$ 0$ & $\$ 0$ & $\$ 0$ & $\$ 0$ & $\$ 0$ & $\$ 0$ \\
\hline Emerging Technologies R\&D & $\$ 1.930$ & $\$ 0$ & $\$ 0$ & $\$ 0$ & $\$ 0$ & $\$ 0$ & $\$ 0$ & $\$ 0$ & $\$ 0$ & $\$ 0$ & $\$ 0$ \\
\hline Emerging Tech R\&D: HPWH & $\$ 0.000$ & $\$ 0$ & $\$ 0$ & $\$ 0$ & $\$ 0$ & $\$ 0$ & $\$ 0$ & $\$ 0$ & $\$ 0$ & $\$ 0$ & $\$ 0$ \\
\hline Emerging Tech R\&D: Roof Top AC & $\$ 0.000$ & $\$ 0$ & $\$ 0$ & $\$ 0$ & $\$ 0$ & $\$ 0$ & $\$ 0$ & $\$ 0$ & $\$ 0$ & $\$ 0$ & $\$ 0$ \\
\hline Emerging Tech R\&D: Gas Condensing WH & $\$ 0.000$ & $\$ 0$ & $\$ 0$ & $\$ 0$ & $\$ 0$ & $\$ 0$ & $\$ 0$ & $\$ 0$ & $\$ 0$ & $\$ 0$ & $\$ 0$ \\
\hline Bldg. Env. R\&D: Windows & $\$ 3.630$ & $\$ 0$ & $\$ 0$ & $\$ 0$ & $\$ 0$ & $\$ 0$ & $\$ 0$ & $\$ 0$ & $\$ 0$ & $\$ 0$ & $\$ 0$ \\
\hline Envelope R\&D: Electrochromic Windows & $\$ 0.000$ & $\$ 0$ & $\$ 0$ & $\$ 0$ & $\$ 0$ & $\$ 0$ & $\$ 0$ & $\$ 0$ & $\$ 0$ & $\$ 0$ & $\$ 0$ \\
\hline Envelope R\&D: Superwindows & $\$ 0.000$ & $\$ 0$ & $\$ 0$ & $\$ 0$ & $\$ 0$ & $\$ 0$ & $\$ 0$ & $\$ 0$ & $\$ 0$ & $\$ 0$ & $\$ 0$ \\
\hline Bldg. Env. R\&D: Roofs and Insulation & $\$ 1.560$ & $\$ 0$ & $\$ 0$ & $\$ 0$ & $\$ 0$ & $\$ 0$ & $\$ 0$ & $\$ 0$ & $\$ 0$ & $\$ 0$ & $\$ 0$ \\
\hline $\begin{array}{l}\text { Envelope R\&D: R30 Insulation/30 Year } \\
\text { Life Roofs }\end{array}$ & $\$ 0.000$ & $\$ 0$ & $\$ 0$ & $\$ 0$ & $\$ 0$ & $\$ 0$ & $\$ 0$ & $\$ 0$ & $\$ 0$ & $\$ 0$ & $\$ 0$ \\
\hline Envelope R\&D: Moisture/Wet Insulation & $\$ 0.000$ & $\$ 0$ & $\$ 0$ & $\$ 0$ & $\$ 0$ & $\$ 0$ & $\$ 0$ & $\$ 0$ & $\$ 0$ & $\$ 0$ & $\$ 0$ \\
\hline Design Strategies and Assistance & $\$ 3.130$ & $\$ 0$ & $\$ 0$ & $\$ 0$ & $\$ 0$ & $\$ 0$ & $\$ 0$ & $\$ 0$ & $\$ 0$ & $\$ 0$ & $\$ 0$ \\
\hline Standards & $\$ 8.930$ & $\$ 0$ & $\$ 0$ & $\$ 0$ & $\$ 0$ & $\$ 0$ & $\$ 0$ & $\$ 0$ & $\$ 0$ & $\$ 0$ & $\$ 0$ \\
\hline Standards: Res Gas Furnaces/Boilers & $\$ 0.000$ & $\$ 0$ & $\$ 0$ & $\$ 0$ & $\$ 0$ & $\$ 0$ & $\$ 0$ & $\$ 0$ & $\$ 0$ & $\$ 0$ & $\$ 0$ \\
\hline Standards: EPACT Standards & $\$ 0.000$ & $\$ 0$ & $\$ 0$ & $\$ 0$ & $\$ 0$ & $\$ 0$ & $\$ 0$ & $\$ 0$ & $\$ 0$ & $\$ 0$ & $\$ 0$ \\
\hline Standards: Dist. Transformers & $\$ 0.000$ & $\$ 0$ & $\$ 0$ & $\$ 0$ & $\$ 0$ & $\$ 0$ & $\$ 0$ & $\$ 0$ & $\$ 0$ & $\$ 0$ & $\$ 0$ \\
\hline Building Research and Standards (DU 5-7) & $\$ 47.940$ & $\$ 0$ & $\$ 0$ & $\$ 0$ & $\$ 0$ & $\$ 0$ & $\$ 0$ & $\$ 0$ & $\$ 0$ & $\$ 0$ & $\$ 0$ \\
\hline All BTS & $\$ 390.030$ & $\$ 47$ & $\$ 47$ & $\$ 45$ & $\$ 45$ & $\$ 45$ & $\$ 46$ & $\$ 47$ & $\$ 47$ & $\$ 47$ & $\$ 47$ \\
\hline
\end{tabular}

Run Title: FY 2003 Run 4 NAS 


\section{NOX Emissions Reductions}


NOx Emissions Reductions (MMTons/yr)

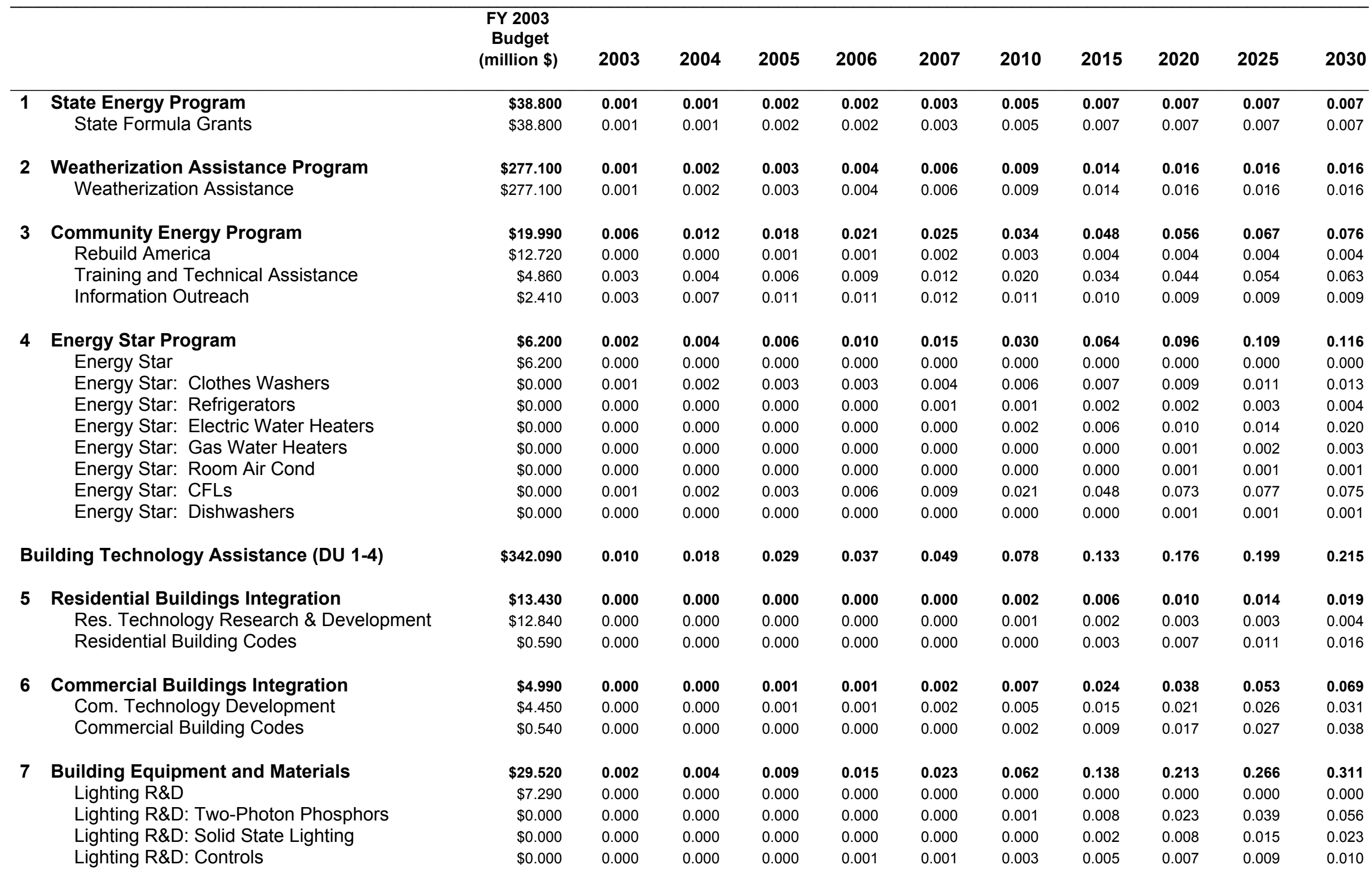

Run Title: FY 2003 Run 4 NAS

MDB: $\quad K: I M E T R I C S I F Y 2003 \mid B E S E T$ InputsIRun 4 NASIFY 2003 Run 4 NAS.mdb

Scenario Last Executed: $12 / 14 / 01 \quad 1: 24: 50$ PM 
NOx Emissions Reductions (MMTons/yr)

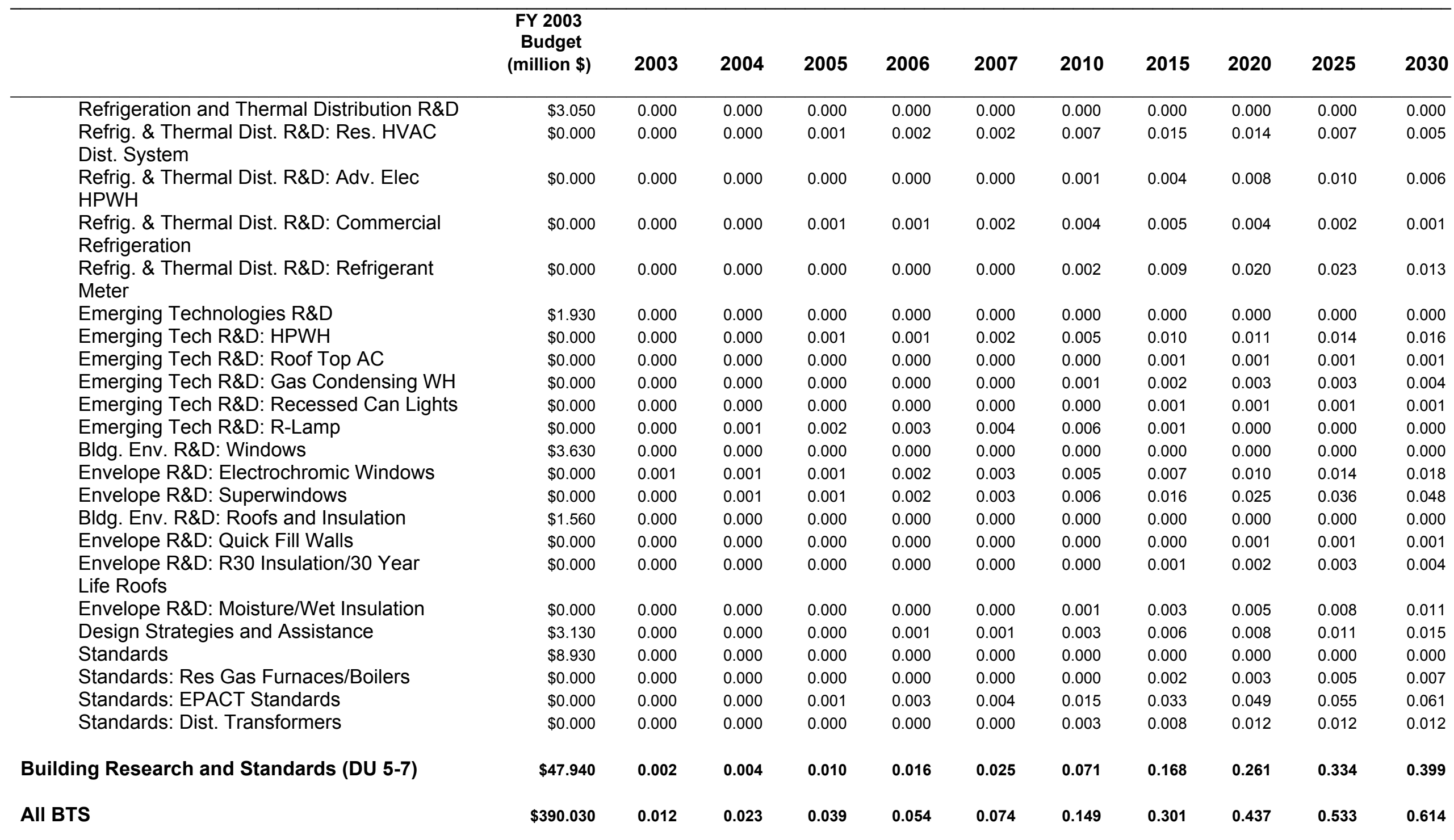

Run Title: FY 2003 Run 4 NAS

Scenario Last Executed: $12 / 14 / 01 \quad 1: 24: 50$ PM

MDB: $\quad$ K:IMETRICSIFY2003IBESET InputsIRun 4 NASIFY 2003 Run 4 NAS.mdb 


\section{PM Emissions Reductions}


PM Emissions Reductions (MMTons/yr)

\begin{tabular}{|c|c|c|c|c|c|c|c|c|c|c|}
\hline $\begin{array}{c}\text { FY } 2003 \\
\text { Budget } \\
\text { (million \$) }\end{array}$ & 2003 & 2004 & 2005 & 2006 & 2007 & 2010 & 2015 & 2020 & 2025 & 2030 \\
\hline$\$ 38.800$ & 0.000 & 0.000 & 0.000 & 0.000 & 0.000 & 0.000 & 0.000 & 0.000 & 0.000 & 0.000 \\
\hline$\$ 38.800$ & 0.000 & 0.000 & 0.000 & 0.000 & 0.000 & 0.000 & 0.000 & 0.000 & 0.000 & 0.000 \\
\hline$\$ 277.100$ & 0.000 & 0.000 & 0.000 & 0.000 & 0.000 & 0.000 & 0.000 & 0.000 & 0.000 & 0.000 \\
\hline$\$ 277.100$ & 0.000 & 0.000 & 0.000 & 0.000 & 0.000 & 0.000 & 0.000 & 0.000 & 0.000 & 0.000 \\
\hline$\$ 19.990$ & 0.000 & 0.000 & 0.001 & 0.001 & 0.001 & 0.001 & 0.001 & 0.002 & 0.002 & 0.002 \\
\hline$\$ 12.720$ & 0.000 & 0.000 & 0.000 & 0.000 & 0.000 & 0.000 & 0.000 & 0.000 & 0.000 & 0.000 \\
\hline$\$ 4.860$ & 0.000 & 0.000 & 0.000 & 0.000 & 0.000 & 0.001 & 0.001 & 0.001 & 0.002 & 0.002 \\
\hline$\$ 2.410$ & 0.000 & 0.000 & 0.000 & 0.000 & 0.000 & 0.000 & 0.000 & 0.000 & 0.000 & 0.000 \\
\hline$\$ 6.200$ & 0.000 & 0.000 & 0.000 & 0.000 & 0.000 & 0.001 & 0.002 & 0.003 & 0.004 & 0.004 \\
\hline$\$ 6.200$ & 0.000 & 0.000 & 0.000 & 0.000 & 0.000 & 0.000 & 0.000 & 0.000 & 0.000 & 0.000 \\
\hline$\$ 0.000$ & 0.000 & 0.000 & 0.000 & 0.000 & 0.000 & 0.000 & 0.000 & 0.000 & 0.000 & 0.000 \\
\hline$\$ 0.000$ & 0.000 & 0.000 & 0.000 & 0.000 & 0.000 & 0.000 & 0.000 & 0.000 & 0.000 & 0.000 \\
\hline$\$ 0.000$ & 0.000 & 0.000 & 0.000 & 0.000 & 0.000 & 0.000 & 0.000 & 0.000 & 0.001 & 0.001 \\
\hline$\$ 0.000$ & 0.000 & 0.000 & 0.000 & 0.000 & 0.000 & 0.000 & 0.000 & 0.000 & 0.000 & 0.000 \\
\hline$\$ 0.000$ & 0.000 & 0.000 & 0.000 & 0.000 & 0.000 & 0.000 & 0.000 & 0.000 & 0.000 & 0.000 \\
\hline$\$ 0.000$ & 0.000 & 0.000 & 0.000 & 0.000 & 0.000 & 0.001 & 0.002 & 0.003 & 0.003 & 0.003 \\
\hline$\$ 0.000$ & 0.000 & 0.000 & 0.000 & 0.000 & 0.000 & 0.000 & 0.000 & 0.000 & 0.000 & 0.000 \\
\hline$\$ 342.090$ & 0.000 & 0.000 & 0.001 & 0.001 & 0.001 & 0.003 & 0.004 & 0.006 & 0.006 & 0.007 \\
\hline$\$ 13.430$ & 0.000 & 0.000 & 0.000 & 0.000 & 0.000 & 0.000 & 0.000 & 0.000 & 0.000 & 0.000 \\
\hline$\$ 12.840$ & 0.000 & 0.000 & 0.000 & 0.000 & 0.000 & 0.000 & 0.000 & 0.000 & 0.000 & 0.000 \\
\hline$\$ 0.590$ & 0.000 & 0.000 & 0.000 & 0.000 & 0.000 & 0.000 & 0.000 & 0.000 & 0.000 & 0.000 \\
\hline$\$ 4.990$ & 0.000 & 0.000 & 0.000 & 0.000 & 0.000 & 0.000 & 0.001 & 0.001 & 0.002 & 0.002 \\
\hline$\$ 4.450$ & 0.000 & 0.000 & 0.000 & 0.000 & 0.000 & 0.000 & 0.000 & 0.001 & 0.001 & 0.001 \\
\hline$\$ 0.540$ & 0.000 & 0.000 & 0.000 & 0.000 & 0.000 & 0.000 & 0.000 & 0.001 & 0.001 & 0.001 \\
\hline$\$ 29.520$ & 0.000 & 0.000 & 0.000 & 0.000 & 0.001 & 0.002 & 0.004 & 0.006 & 0.008 & 0.009 \\
\hline$\$ 7.290$ & 0.000 & 0.000 & 0.000 & 0.000 & 0.000 & 0.000 & 0.000 & 0.000 & 0.000 & 0.000 \\
\hline$\$ 0.000$ & 0.000 & 0.000 & 0.000 & 0.000 & 0.000 & 0.000 & 0.000 & 0.001 & 0.001 & 0.002 \\
\hline$\$ 0.000$ & 0.000 & 0.000 & 0.000 & 0.000 & 0.000 & 0.000 & 0.000 & 0.000 & 0.001 & 0.001 \\
\hline$\$ 0.000$ & 0.000 & 0.000 & 0.000 & 0.000 & 0.000 & 0.000 & 0.000 & 0.000 & 0.000 & 0.000 \\
\hline$\$ 3.050$ & 0.000 & 0.000 & 0.000 & 0.000 & 0.000 & 0.000 & 0.000 & 0.000 & 0.000 & 0.000 \\
\hline$\$ 0.000$ & 0.000 & 0.000 & 0.000 & 0.000 & 0.000 & 0.000 & 0.000 & 0.000 & 0.000 & 0.000 \\
\hline$\$ 0.000$ & 0.000 & 0.000 & 0.000 & 0.000 & 0.000 & 0.000 & 0.000 & 0.000 & 0.000 & 0.000 \\
\hline
\end{tabular}

\footnotetext{
Run Title: FY 2003 Run 4 NAS

Scenario Last Executed:

$12 / 14 / 01$

$1: 24: 50$ PM 
PM Emissions Reductions (MMTons/yr)

\begin{tabular}{|c|c|c|c|c|c|c|c|c|c|c|}
\hline $\begin{array}{c}\text { FY } 2003 \\
\text { Budget } \\
\text { (million \$) }\end{array}$ & 2003 & 2004 & 2005 & 2006 & 2007 & 2010 & 2015 & 2020 & 2025 & 2030 \\
\hline$\$ 0.000$ & 0.000 & 0.000 & 0.000 & 0.000 & 0.000 & 0.000 & 0.000 & 0.000 & 0.000 & 0.000 \\
\hline$\$ 0.000$ & 0.000 & 0.000 & 0.000 & 0.000 & 0.000 & 0.000 & 0.000 & 0.001 & 0.001 & 0.000 \\
\hline$\$ 1.930$ & 0.000 & 0.000 & 0.000 & 0.000 & 0.000 & 0.000 & 0.000 & 0.000 & 0.000 & 0.000 \\
\hline$\$ 0.000$ & 0.000 & 0.000 & 0.000 & 0.000 & 0.000 & 0.000 & 0.000 & 0.000 & 0.000 & 0.001 \\
\hline$\$ 0.000$ & 0.000 & 0.000 & 0.000 & 0.000 & 0.000 & 0.000 & 0.000 & 0.000 & 0.000 & 0.000 \\
\hline$\$ 0.000$ & 0.000 & 0.000 & 0.000 & 0.000 & 0.000 & 0.000 & 0.000 & 0.000 & 0.000 & 0.000 \\
\hline$\$ 0.000$ & 0.000 & 0.000 & 0.000 & 0.000 & 0.000 & 0.000 & 0.000 & 0.000 & 0.000 & 0.000 \\
\hline$\$ 0.000$ & 0.000 & 0.000 & 0.000 & 0.000 & 0.000 & 0.000 & 0.000 & 0.000 & 0.000 & 0.000 \\
\hline$\$ 3.630$ & 0.000 & 0.000 & 0.000 & 0.000 & 0.000 & 0.000 & 0.000 & 0.000 & 0.000 & 0.000 \\
\hline$\$ 0.000$ & 0.000 & 0.000 & 0.000 & 0.000 & 0.000 & 0.000 & 0.000 & 0.000 & 0.000 & 0.001 \\
\hline$\$ 0.000$ & 0.000 & 0.000 & 0.000 & 0.000 & 0.000 & 0.000 & 0.000 & 0.001 & 0.001 & 0.001 \\
\hline$\$ 1.560$ & 0.000 & 0.000 & 0.000 & 0.000 & 0.000 & 0.000 & 0.000 & 0.000 & 0.000 & 0.000 \\
\hline$\$ 0.000$ & 0.000 & 0.000 & 0.000 & 0.000 & 0.000 & 0.000 & 0.000 & 0.000 & 0.000 & 0.000 \\
\hline$\$ 0.000$ & 0.000 & 0.000 & 0.000 & 0.000 & 0.000 & 0.000 & 0.000 & 0.000 & 0.000 & 0.000 \\
\hline$\$ 0.000$ & 0.000 & 0.000 & 0.000 & 0.000 & 0.000 & 0.000 & 0.000 & 0.000 & 0.000 & 0.000 \\
\hline$\$ 3.130$ & 0.000 & 0.000 & 0.000 & 0.000 & 0.000 & 0.000 & 0.000 & 0.000 & 0.000 & 0.000 \\
\hline$\$ 8.930$ & 0.000 & 0.000 & 0.000 & 0.000 & 0.000 & 0.000 & 0.000 & 0.000 & 0.000 & 0.000 \\
\hline$\$ 0.000$ & 0.000 & 0.000 & 0.000 & 0.000 & 0.000 & 0.000 & 0.000 & 0.000 & 0.000 & 0.000 \\
\hline$\$ 0.000$ & 0.000 & 0.000 & 0.000 & 0.000 & 0.000 & 0.001 & 0.001 & 0.002 & 0.002 & 0.002 \\
\hline$\$ 0.000$ & 0.000 & 0.000 & 0.000 & 0.000 & 0.000 & 0.000 & 0.000 & 0.000 & 0.000 & 0.000 \\
\hline$\$ 47.940$ & 0.000 & 0.000 & 0.000 & 0.000 & 0.001 & 0.002 & 0.005 & 0.008 & 0.010 & 0.012 \\
\hline$\$ 390.030$ & 0.000 & 0.001 & 0.001 & 0.001 & 0.002 & 0.005 & 0.009 & 0.014 & 0.016 & 0.019 \\
\hline
\end{tabular}

HPWH

Refrig. \& Thermal Dist. R\&D: Commercial

Refrigeration

Refrig. \& Thermal Dist. R\&D: Refrigerant Meter

Emerging Technologies R\&D

Emerging Tech R\&D: HPWH

Emerging Tech R\&D: Roof Top AC

Emerging Tech R\&D: Gas Condensing WH

Emerging Tech R\&D: Recessed Can Lights

Emerging Tech R\&D: R-Lamp

Bldg. Env. R\&D: Windows

Envelope R\&D: Electrochromic Windows

Envelope R\&D: Superwindows

Bldg. Env. R\&D: Roofs and Insulation

Envelope R\&D: Quick Fill Walls

Envelope R\&D: R30 Insulation/30 Year

Life Roofs

Envelope R\&D: Moisture/Wet Insulation

Design Strategies and Assistance

Standards

Standards: Res Gas Furnaces/Boilers

Standards: EPACT Standards

Standards: Dist. Transformers

Building Research and Standards (DU 5-7)

All BTS $\begin{array}{lll}0.000 & 0.001 & 0.001\end{array}$

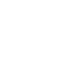




\section{Total Primary Energy Savings}


Primary Energy Savings (TBtu/yr)

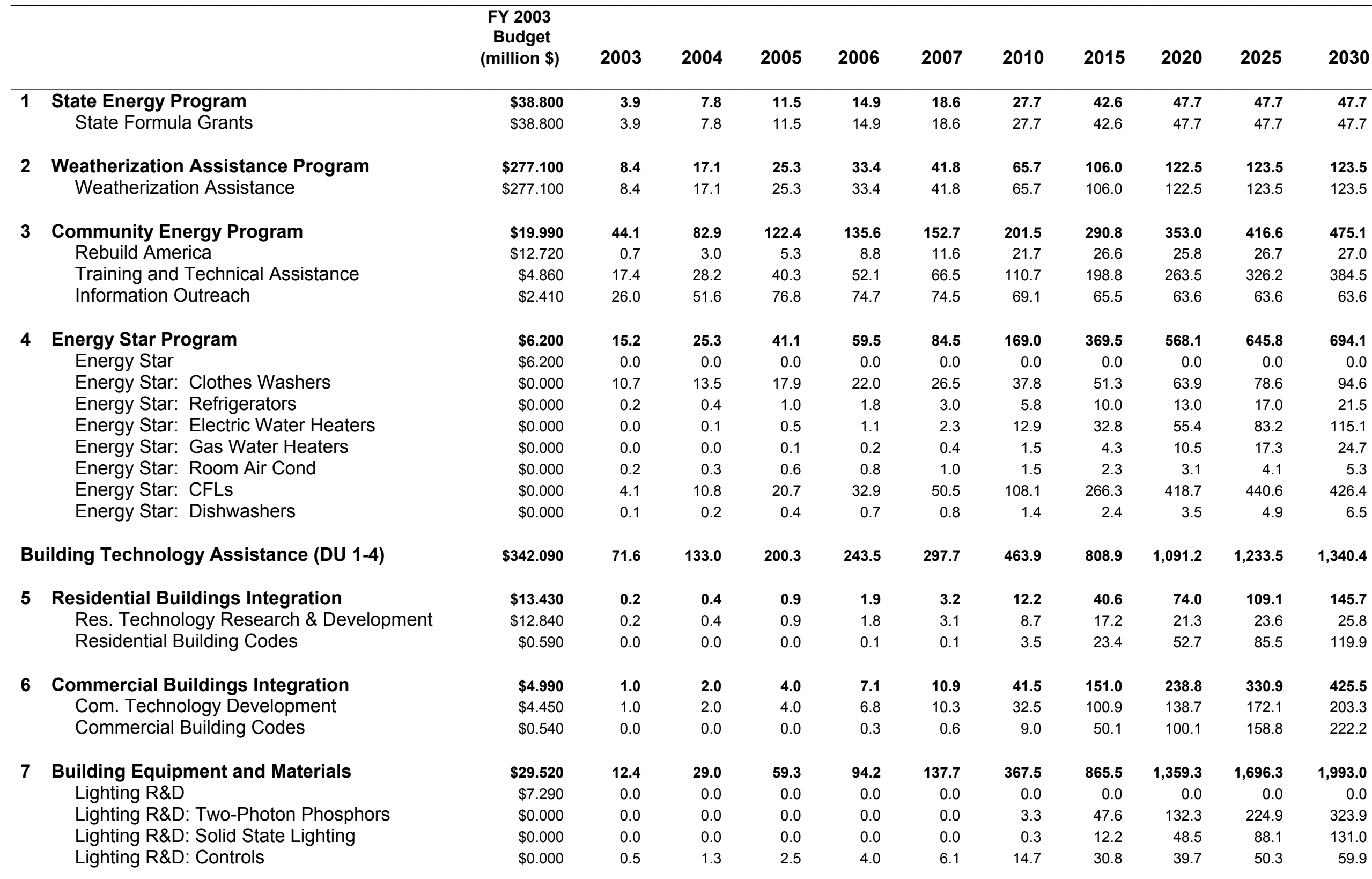

Run Title: FY 2003 Run 4 NAS

Scenario Last Executed: 12/14/01 1:24:50 PM

MDB: $\quad$ K:IMETRICSIFY2003\BESET Inputs\Run 4 NASIFY 2003 Run 4 NAS.mdb 
Primary Energy Savings (TBtu/yr)

\begin{tabular}{|c|c|c|c|c|c|c|c|c|c|c|c|}
\hline & $\begin{array}{c}\text { FY } 2003 \\
\text { Budget } \\
\text { (million \$) }\end{array}$ & 2003 & 2004 & 2005 & 2006 & 2007 & 2010 & 2015 & 2020 & 2025 & 2030 \\
\hline Refrigeration and Thermal Distribution R\&D & $\$ 3.050$ & 0.0 & 0.0 & 0.0 & 0.0 & 0.0 & 0.0 & 0.0 & 0.0 & 0.0 & 0.0 \\
\hline $\begin{array}{l}\text { Refrig. \& Thermal Dist. R\&D: Res. HVAC } \\
\text { Dist. System }\end{array}$ & $\$ 0.000$ & 1.6 & 3.8 & 7.0 & 11.4 & 17.5 & 47.9 & 110.6 & 101.8 & 49.8 & 35.1 \\
\hline $\begin{array}{l}\text { Refrig. \& Thermal Dist. R\&D: Adv. Elec } \\
\text { HPWH }\end{array}$ & $\$ 0.000$ & 0.0 & 0.0 & 0.1 & 0.3 & 0.8 & 3.5 & 20.0 & 47.5 & 57.5 & 34.9 \\
\hline $\begin{array}{l}\text { Refrig. \& Thermal Dist. R\&D: Refrigerant } \\
\text { Meter }\end{array}$ & $\$ 0.000$ & 0.0 & 0.0 & 0.3 & 0.9 & 2.0 & 9.7 & 52.2 & 116.6 & 133.9 & 76.3 \\
\hline Emerging Technologies R\&D & $\$ 1.930$ & 0.0 & 0.0 & 0.0 & 0.0 & 0.0 & 0.0 & 0.0 & 0.0 & 0.0 & 0.0 \\
\hline Emerging Tech R\&D: HPWH & $\$ 0.000$ & 0.0 & 2.1 & 3.4 & 5.3 & 8.3 & 24.6 & 55.5 & 65.2 & 79.0 & 94.5 \\
\hline Emerging Tech R\&D: Roof Top AC & $\$ 0.000$ & 0.0 & 0.0 & 0.7 & 1.2 & 1.6 & 2.3 & 3.0 & 3.4 & 4.0 & 4.6 \\
\hline Emerging Tech R\&D: Gas Condensing WH & $\$ 0.000$ & 0.0 & 0.0 & 0.2 & 0.6 & 1.2 & 7.0 & 21.0 & 25.3 & 30.1 & 35.2 \\
\hline Emerging Tech R\&D: Recessed Can Lights & $\$ 0.000$ & 0.1 & 0.1 & 0.3 & 0.4 & 0.6 & 1.5 & 3.3 & 3.9 & 3.9 & 3.9 \\
\hline Bldg. Env. R\&D: Windows & $\$ 3.630$ & 0.0 & 0.0 & 0.0 & 0.0 & 0.0 & 0.0 & 0.0 & 0.0 & 0.0 & 0.0 \\
\hline Envelope R\&D: Electrochromic Windows & $\$ 0.000$ & 3.9 & 6.0 & 8.6 & 11.3 & 14.6 & 23.8 & 38.2 & 56.2 & 78.2 & 102.5 \\
\hline Envelope R\&D: Superwindows & $\$ 0.000$ & 2.4 & 5.5 & 9.6 & 14.3 & 20.4 & 46.9 & 122.1 & 194.2 & 274.3 & 361.7 \\
\hline Bldg. Env. R\&D: Roofs and Insulation & $\$ 1.560$ & 0.0 & 0.0 & 0.0 & 0.0 & 0.0 & 0.0 & 0.0 & 0.0 & 0.0 & 0.0 \\
\hline Envelope R\&D: Quick Fill Walls & $\$ 0.000$ & 0.0 & 0.0 & 0.1 & 0.1 & 0.2 & 0.5 & 2.3 & 5.1 & 8.3 & 11.8 \\
\hline $\begin{array}{l}\text { Envelope R\&D: R30 Insulation/30 Year } \\
\text { Life Roofs }\end{array}$ & $\$ 0.000$ & 0.0 & 0.0 & 0.0 & 0.0 & 0.0 & 0.3 & 4.4 & 14.3 & 24.5 & 35.1 \\
\hline Envelope R\&D: Moisture/Wet Insulation & $\$ 0.000$ & 0.0 & 0.2 & 0.8 & 1.6 & 2.8 & 8.2 & 22.2 & 42.7 & 65.7 & 91.0 \\
\hline Design Strategies and Assistance & $\$ 3.130$ & 0.7 & 1.6 & 3.0 & 4.7 & 7.0 & 16.2 & 36.5 & 53.2 & 73.9 & 94.4 \\
\hline Standards & $\$ 8.930$ & 0.0 & 0.0 & 0.0 & 0.0 & 0.0 & 0.0 & 0.0 & 0.0 & 0.0 & 0.0 \\
\hline Standards: Res Gas Furnaces/Boilers & $\$ 0.000$ & 0.0 & 0.0 & 0.0 & 0.0 & 0.0 & 4.7 & 17.4 & 30.9 & 45.7 & 62.1 \\
\hline Standards: EPACT Standards & $\$ 0.000$ & 0.0 & 0.0 & 7.8 & 14.9 & 23.4 & 83.2 & 188.4 & 287.4 & 325.2 & 363.0 \\
\hline Standards: Dist. Transformers & $\$ 0.000$ & 0.0 & 0.0 & 0.0 & 0.0 & 0.0 & 18.2 & 44.5 & 68.8 & 68.8 & 68.8 \\
\hline Building Research and Standards (DU 5-7) & $\$ 47.940$ & 13.6 & 31.5 & 64.3 & 103.2 & 151.8 & 421.3 & $1,057.2$ & $1,672.1$ & $2,136.3$ & $2,564.2$ \\
\hline All BTS & $\$ 390.030$ & 85.2 & 164.5 & 264.6 & 346.7 & 449.4 & 885.1 & $1,866.1$ & $2,763.3$ & $3,369.8$ & $3,904.5$ \\
\hline
\end{tabular}

Run Title: FY 2003 Run 4 NAS

Scenario Last Executed: $12 / 14 / 01 \quad 1: 24: 50$ PM

MDB: $\quad$ K:IMETRICSIFY2003IBESET InputsIRun 4 NASIFY 2003 Run 4 NAS.mdb 


\section{Primary Electricity Savings}




\section{Primary Electricity Energy Savings (TBtu/Year)}

\begin{tabular}{|c|c|c|c|c|c|c|c|c|c|c|c|c|}
\hline & & $\begin{array}{c}\text { FY } 2003 \\
\text { Budget } \\
\text { (million \$) }\end{array}$ & 2003 & 2004 & 2005 & 2006 & 2007 & 2010 & 2015 & 2020 & 2025 & 2030 \\
\hline \multirow[t]{2}{*}{1} & State Energy Program & $\$ 38.800$ & 2.7 & 5.3 & 7.8 & 10.0 & 12.5 & 17.8 & 26.6 & 29.2 & 29.2 & 29.2 \\
\hline & State Formula Grants & $\$ 38.800$ & 2.7 & 5.3 & 7.8 & 10.0 & 12.5 & 17.8 & 26.6 & 29.2 & 29.2 & 29.2 \\
\hline \multirow[t]{2}{*}{2} & Weatherization Assistance Program & $\$ 277.100$ & 2.1 & 4.2 & 6.2 & 8.0 & 9.9 & 14.3 & 21.5 & 23.9 & 24.1 & 24.1 \\
\hline & Weatherization Assistance & $\$ 277.100$ & 2.1 & 4.2 & 6.2 & 8.0 & 9.9 & 14.3 & 21.5 & 23.9 & 24.1 & 24.1 \\
\hline \multirow[t]{4}{*}{3} & Community Energy Program & $\$ 19.990$ & 34.7 & 63.8 & 93.6 & 104.3 & 119.3 & 158.6 & 232.0 & 283.3 & 337.0 & 386.9 \\
\hline & Rebuild America & $\$ 12.720$ & 0.4 & 1.6 & 2.7 & 4.5 & 6.0 & 10.5 & 12.5 & 12.1 & 12.6 & 12.9 \\
\hline & Training and Technical Assistance & $\$ 4.860$ & 16.7 & 27.3 & 39.1 & 50.2 & 63.9 & 104.0 & 179.1 & 232.6 & 285.7 & 335.4 \\
\hline & Information Outreach & $\$ 2.410$ & 17.6 & 34.9 & 51.8 & 49.6 & 49.4 & 44.1 & 40.4 & 38.6 & 38.6 & 38.6 \\
\hline \multirow[t]{9}{*}{4} & Energy Star Program & $\$ 6.200$ & 11.0 & 20.1 & 34.2 & 50.8 & 74.1 & 153.5 & 349.7 & 540.8 & 604.5 & 636.4 \\
\hline & Energy Star & $\$ 6.200$ & 0.0 & 0.0 & 0.0 & 0.0 & 0.0 & 0.0 & 0.0 & 0.0 & 0.0 & 0.0 \\
\hline & Energy Star: Clothes Washers & $\$ 0.000$ & 6.4 & 8.0 & 10.4 & 12.5 & 15.0 & 20.2 & 26.1 & 31.1 & 37.9 & 45.5 \\
\hline & Energy Star: Refrigerators & $\$ 0.000$ & 0.2 & 0.4 & 1.0 & 1.8 & 3.0 & 5.8 & 10.0 & 13.0 & 17.0 & 21.5 \\
\hline & Energy Star: Electric Water Heaters & $\$ 0.000$ & 0.0 & 0.1 & 0.5 & 1.1 & 2.3 & 12.9 & 32.8 & 55.4 & 83.2 & 115.1 \\
\hline & Energy Star: Gas Water Heaters & $\$ 0.000$ & 0.0 & 0.0 & 0.0 & 0.0 & 0.0 & 0.0 & 0.0 & 0.0 & 0.0 & 0.0 \\
\hline & Energy Star: Room Air Cond & $\$ 0.000$ & 0.2 & 0.3 & 0.6 & 0.8 & 1.0 & 1.5 & 2.3 & 3.1 & 4.1 & 5.3 \\
\hline & Energy Star: CFLs & $\$ 0.000$ & 4.2 & 11.1 & 21.3 & 33.9 & 52.0 & 111.7 & 276.0 & 434.6 & 457.3 & 442.6 \\
\hline & Energy Star: Dishwashers & $\$ 0.000$ & 0.1 & 0.2 & 0.4 & 0.7 & 0.8 & 1.4 & 2.4 & 3.5 & 4.9 & 6.5 \\
\hline \multicolumn{2}{|r|}{ Building Technology Assistance (DU 1-4) } & $\$ 342.090$ & 50.5 & 93.5 & 141.8 & 173.1 & 215.8 & 344.2 & 629.7 & 877.2 & 994.9 & $1,076.7$ \\
\hline \multirow[t]{3}{*}{5} & Residential Buildings Integration & $\$ 13.430$ & 0.1 & 0.2 & 0.4 & 0.8 & 1.4 & 5.1 & 15.7 & 26.9 & 38.5 & 50.6 \\
\hline & Res. Technology Research \& Development & $\$ 12.840$ & 0.1 & 0.2 & 0.4 & 0.8 & 1.4 & 3.8 & 7.4 & 9.3 & 10.3 & 11.3 \\
\hline & Residential Building Codes & $\$ 0.590$ & 0.0 & 0.0 & 0.0 & 0.0 & 0.0 & 1.3 & 8.2 & 17.6 & 28.2 & 39.3 \\
\hline \multirow[t]{3}{*}{6} & Commercial Buildings Integration & $\$ 4.990$ & 0.6 & 1.3 & 2.6 & 4.7 & 7.2 & 29.3 & 112.6 & 187.5 & 271.1 & 358.1 \\
\hline & Com. Technology Development & $\$ 4.450$ & 0.6 & 1.3 & 2.6 & 4.4 & 6.6 & 20.3 & 62.6 & 87.5 & 112.4 & 136.1 \\
\hline & Commercial Building Codes & $\$ 0.540$ & 0.0 & 0.0 & 0.0 & 0.3 & 0.6 & 9.0 & 50.1 & 100.0 & 158.7 & 222.0 \\
\hline \multirow[t]{8}{*}{7} & Building Equipment and Materials & $\$ 29.520$ & 10.4 & 23.5 & 48.5 & 76.4 & 110.4 & 282.4 & 633.9 & $1,027.7$ & $1,286.4$ & $1,474.3$ \\
\hline & Lighting R\&D & $\$ 7.290$ & 0.0 & 0.0 & 0.0 & 0.0 & 0.0 & 0.0 & 0.0 & 0.0 & 0.0 & 0.0 \\
\hline & Lighting R\&D: Two-Photon Phosphors & $\$ 0.000$ & 0.0 & 0.0 & 0.0 & 0.0 & 0.0 & 3.3 & 47.6 & 132.3 & 224.9 & 323.9 \\
\hline & Lighting R\&D: Solid State Lighting & $\$ 0.000$ & 0.0 & 0.0 & 0.0 & 0.0 & 0.0 & 0.3 & 12.2 & 48.5 & 88.1 & 131.0 \\
\hline & Lighting R\&D: Controls & $\$ 0.000$ & 0.5 & 1.3 & 2.5 & 4.0 & 6.1 & 14.7 & 30.8 & 39.7 & 50.3 & 59.9 \\
\hline & Refrigeration and Thermal Distribution R\&D & $\$ 3.050$ & 0.0 & 0.0 & 0.0 & 0.0 & 0.0 & 0.0 & 0.0 & 0.0 & 0.0 & 0.0 \\
\hline & $\begin{array}{l}\text { Refrig. \& Thermal Dist. R\&D: Res. HVAC } \\
\text { Dist. System }\end{array}$ & $\$ 0.000$ & 0.7 & 1.7 & 3.2 & 5.1 & 7.8 & 20.0 & 44.1 & 40.4 & 21.3 & 16.6 \\
\hline & $\begin{array}{l}\text { Refrig. \& Thermal Dist. R\&D: Adv. Elec } \\
\text { HPWH }\end{array}$ & $\$ 0.000$ & 0.0 & 0.0 & 0.1 & 0.3 & 0.8 & 3.5 & 20.0 & 47.5 & 57.5 & 34.9 \\
\hline
\end{tabular}

Run Title: FY 2003 Run 4 NAS 


\section{Primary Electricity Energy Savings (TBtu/Year)}

\begin{tabular}{|c|c|c|c|c|c|c|c|c|c|c|c|}
\hline & $\begin{array}{c}\text { FY } 2003 \\
\text { Budget } \\
\text { (million \$) }\end{array}$ & 2003 & 2004 & 2005 & 2006 & 2007 & 2010 & 2015 & 2020 & 2025 & 2030 \\
\hline $\begin{array}{l}\text { Refrig. \& Thermal Dist. R\&D: Commercial } \\
\text { Refrigeration }\end{array}$ & $\$ 0.000$ & 1.3 & 2.9 & 4.9 & 7.1 & 9.8 & 18.1 & 27.1 & 20.6 & 9.4 & 3.0 \\
\hline $\begin{array}{l}\text { Refrig. \& Thermal Dist. R\&D: Refrigerant } \\
\text { Meter }\end{array}$ & $\$ 0.000$ & 0.0 & 0.0 & 0.3 & 0.9 & 2.0 & 9.7 & 52.2 & 116.6 & 133.9 & 76.3 \\
\hline Emerging Technologies R\&D & $\$ 1.930$ & 0.0 & 0.0 & 0.0 & 0.0 & 0.0 & 0.0 & 0.0 & 0.0 & 0.0 & 0.0 \\
\hline Emerging Tech R\&D: HPWH & $\$ 0.000$ & 0.0 & 2.1 & 3.4 & 5.3 & 8.3 & 24.6 & 55.5 & 65.2 & 79.0 & 94.5 \\
\hline Emerging Tech R\&D: Roof Top AC & $\$ 0.000$ & 0.0 & 0.0 & 0.7 & 1.2 & 1.6 & 2.3 & 3.0 & 3.4 & 4.0 & 4.6 \\
\hline Emerging Tech R\&D: Gas Condensing WH & $\$ 0.000$ & 0.0 & 0.0 & 0.0 & 0.0 & 0.0 & 0.0 & 0.0 & 0.0 & 0.0 & 0.0 \\
\hline Emerging Tech R\&D: Recessed Can Lights & $\$ 0.000$ & 0.1 & 0.2 & 0.3 & 0.4 & 0.7 & 1.6 & 3.4 & 4.0 & 4.0 & 4.0 \\
\hline Emerging Tech R\&D: R-Lamp & $\$ 0.000$ & 2.0 & 5.3 & 10.2 & 16.2 & 21.3 & 32.3 & 4.3 & 0.0 & 0.0 & 0.0 \\
\hline Bldg. Env. R\&D: Windows & $\$ 3.630$ & 0.0 & 0.0 & 0.0 & 0.0 & 0.0 & 0.0 & 0.0 & 0.0 & 0.0 & 0.0 \\
\hline Envelope R\&D: Electrochromic Windows & $\$ 0.000$ & 4.6 & 7.0 & 10.0 & 13.0 & 16.6 & 26.6 & 41.5 & 59.6 & 81.6 & 105.8 \\
\hline Envelope R\&D: Superwindows & $\$ 0.000$ & 0.7 & 1.7 & 2.9 & 4.4 & 6.4 & 14.2 & 36.8 & 59.7 & 88.1 & 120.5 \\
\hline Bldg. Env. R\&D: Roofs and Insulation & $\$ 1.560$ & 0.0 & 0.0 & 0.0 & 0.0 & 0.0 & 0.0 & 0.0 & 0.0 & 0.0 & 0.0 \\
\hline Envelope R\&D: Quick Fill Walls & $\$ 0.000$ & 0.0 & 0.0 & 0.0 & 0.0 & 0.0 & 0.1 & 0.4 & 0.9 & 1.5 & 2.2 \\
\hline $\begin{array}{l}\text { Envelope R\&D: R30 Insulation/30 Year } \\
\text { Life Roofs }\end{array}$ & $\$ 0.000$ & 0.0 & 0.0 & 0.0 & 0.0 & 0.0 & 0.1 & 0.7 & 1.6 & 2.7 & 3.9 \\
\hline Envelope R\&D: Moisture/Wet Insulation & $\$ 0.000$ & 0.0 & 0.0 & 0.1 & 0.1 & 0.1 & 0.5 & 2.3 & 5.5 & 9.4 & 13.8 \\
\hline Design Strategies and Assistance & $\$ 3.130$ & 0.5 & 1.3 & 2.3 & 3.7 & 5.5 & 12.2 & 27.0 & 39.0 & 54.2 & 69.3 \\
\hline Standards & $\$ 8.930$ & 0.0 & 0.0 & 0.0 & 0.0 & 0.0 & 0.0 & 0.0 & 0.0 & 0.0 & 0.0 \\
\hline Standards: Res Gas Furnaces/Boilers & $\$ 0.000$ & 0.0 & 0.0 & 0.0 & 0.0 & 0.0 & 0.0 & -0.1 & -0.1 & -0.2 & -0.3 \\
\hline Standards: EPACT Standards & $\$ 0.000$ & 0.0 & 0.0 & 7.8 & 14.9 & 23.4 & 80.3 & 180.5 & 274.4 & 307.9 & 341.4 \\
\hline Standards: Dist. Transformers & $\$ 0.000$ & 0.0 & 0.0 & 0.0 & 0.0 & 0.0 & 18.2 & 44.5 & 68.8 & 68.8 & 68.8 \\
\hline Building Research and Standards (DU 5-7) & $\$ 47.940$ & 11.1 & 25.1 & 51.6 & 81.9 & 119.0 & 316.8 & 762.2 & $1,242.1$ & $1,596.0$ & $1,882.9$ \\
\hline All BTS & $\$ 390.030$ & 61.6 & 118.6 & 193.4 & 255.0 & 334.9 & 661.0 & $1,391.9$ & $2,119.3$ & $2,590.9$ & $2,959.7$ \\
\hline
\end{tabular}

Run Title: FY 2003 Run 4 NAS 


\section{Primary Non-Electric Savings}


Primary Non-Electric Energy Savings (TBtu/yr)

\begin{tabular}{|c|c|c|c|c|c|c|c|c|c|c|c|c|}
\hline & & $\begin{array}{c}\text { FY } 2003 \\
\text { Budget } \\
\text { (Million \$) }\end{array}$ & 2003 & 2004 & 2005 & 2006 & 2007 & 2010 & 2015 & 2020 & 2025 & 2030 \\
\hline \multirow[t]{2}{*}{1} & State Energy Program & $\$ 38.800$ & 1.2 & 2.5 & 3.7 & 4.9 & 6.2 & 9.8 & 16.0 & 18.4 & 18.4 & 18.4 \\
\hline & State Formula Grants & $\$ 38.800$ & 1.2 & 2.5 & 3.7 & 4.9 & 6.2 & 9.8 & 16.0 & 18.4 & 18.4 & 18.4 \\
\hline \multirow[t]{2}{*}{2} & Weatherization Assistance Program & $\$ 277.100$ & 6.3 & 12.8 & 19.1 & 25.5 & 31.9 & 51.4 & 84.6 & 98.6 & 99.4 & 99.4 \\
\hline & Weatherization Assistance & $\$ 277.100$ & 6.3 & 12.8 & 19.1 & 25.5 & 31.9 & 51.4 & 84.6 & 98.6 & 99.4 & 99.4 \\
\hline \multirow[t]{4}{*}{3} & Community Energy Program & $\$ 19.990$ & 9.4 & 19.0 & 28.8 & 31.3 & 33.3 & 43.0 & 58.8 & 69.7 & 79.6 & 88.2 \\
\hline & Rebuild America & $\$ 12.720$ & 0.3 & 1.4 & 2.5 & 4.3 & 5.7 & 11.2 & 14.1 & 13.8 & 14.0 & 14.0 \\
\hline & Training and Technical Assistance & $\$ 4.860$ & 0.7 & 0.9 & 1.2 & 1.9 & 2.6 & 6.7 & 19.7 & 30.9 & 40.5 & 49.1 \\
\hline & Information Outreach & $\$ 2.410$ & 8.4 & 16.7 & 25.1 & 25.1 & 25.1 & 25.1 & 25.1 & 25.1 & 25.1 & 25.1 \\
\hline \multirow[t]{9}{*}{4} & Energy Star Program & $\$ 6.200$ & 4.2 & 5.2 & 6.9 & 8.7 & 10.4 & 15.5 & 19.8 & 27.3 & 41.2 & 57.6 \\
\hline & Energy Star & $\$ 6.200$ & 0.0 & 0.0 & 0.0 & 0.0 & 0.0 & 0.0 & 0.0 & 0.0 & 0.0 & 0.0 \\
\hline & Energy Star: Clothes Washers & $\$ 0.000$ & 4.3 & 5.5 & 7.5 & 9.5 & 11.5 & 17.6 & 25.2 & 32.8 & 40.7 & 49.1 \\
\hline & Energy Star: Refrigerators & $\$ 0.000$ & 0.0 & 0.0 & 0.0 & 0.0 & 0.0 & 0.0 & 0.0 & 0.0 & 0.0 & 0.0 \\
\hline & Energy Star: Electric Water Heaters & $\$ 0.000$ & 0.0 & 0.0 & 0.0 & 0.0 & 0.0 & 0.0 & 0.0 & 0.0 & 0.0 & 0.0 \\
\hline & Energy Star: Gas Water Heaters & $\$ 0.000$ & 0.0 & 0.0 & 0.1 & 0.2 & 0.4 & 1.5 & 4.3 & 10.5 & 17.3 & 24.7 \\
\hline & Energy Star: Room Air Cond & $\$ 0.000$ & 0.0 & 0.0 & 0.0 & 0.0 & 0.0 & 0.0 & 0.0 & 0.0 & 0.0 & 0.0 \\
\hline & Energy Star: CFLs & $\$ 0.000$ & -0.1 & -0.3 & -0.6 & -1.0 & -1.5 & -3.6 & -9.7 & -15.9 & -16.7 & -16.2 \\
\hline & Energy Star: Dishwashers & $\$ 0.000$ & 0.0 & 0.0 & 0.0 & 0.0 & 0.0 & 0.0 & 0.0 & 0.0 & 0.0 & 0.0 \\
\hline \multicolumn{2}{|r|}{ Building Technology Assistance (DU 1-4) } & $\$ 342.090$ & 21.2 & 39.5 & 58.6 & 70.4 & 81.8 & 119.7 & 179.2 & 214.0 & 238.7 & 263.6 \\
\hline \multirow[t]{3}{*}{5} & Residential Buildings Integration & $\$ 13.430$ & 0.1 & 0.2 & 0.5 & 1.1 & 1.8 & 7.1 & 24.9 & 47.1 & 70.6 & 95.1 \\
\hline & Res. Technology Research \& Development & $\$ 12.840$ & 0.1 & 0.2 & 0.5 & 1.0 & 1.7 & 4.9 & 9.7 & 12.0 & 13.3 & 14.5 \\
\hline & Residential Building Codes & $\$ 0.590$ & 0.0 & 0.0 & 0.0 & 0.1 & 0.1 & 2.2 & 15.2 & 35.1 & 57.3 & 80.6 \\
\hline \multirow[t]{3}{*}{6} & Commercial Buildings Integration & $\$ 4.990$ & 0.4 & 0.7 & 1.4 & 2.4 & 3.6 & 12.2 & 38.4 & 51.2 & 59.8 & 67.4 \\
\hline & Com. Technology Development & $\$ 4.450$ & 0.4 & 0.7 & 1.4 & 2.4 & 3.6 & 12.2 & 38.4 & 51.1 & 59.7 & 67.2 \\
\hline & Commercial Building Codes & $\$ 0.540$ & 0.0 & 0.0 & 0.0 & 0.0 & 0.0 & 0.0 & 0.0 & 0.1 & 0.1 & 0.2 \\
\hline \multirow[t]{8}{*}{7} & Building Equipment and Materials & $\$ 29.520$ & 2.0 & 5.5 & 10.8 & 17.8 & 27.3 & 85.1 & 231.6 & 331.7 & 410.0 & 518.7 \\
\hline & Lighting R\&D & $\$ 7.290$ & 0.0 & 0.0 & 0.0 & 0.0 & 0.0 & 0.0 & 0.0 & 0.0 & 0.0 & 0.0 \\
\hline & Lighting R\&D: Two-Photon Phosphors & $\$ 0.000$ & 0.0 & 0.0 & 0.0 & 0.0 & 0.0 & 0.0 & 0.0 & 0.0 & 0.0 & 0.0 \\
\hline & Lighting R\&D: Solid State Lighting & $\$ 0.000$ & 0.0 & 0.0 & 0.0 & 0.0 & 0.0 & 0.0 & 0.0 & 0.0 & 0.0 & 0.0 \\
\hline & Lighting R\&D: Controls & $\$ 0.000$ & 0.0 & 0.0 & 0.0 & 0.0 & 0.0 & 0.0 & 0.0 & 0.0 & 0.0 & 0.0 \\
\hline & Refrigeration and Thermal Distribution R\&D & $\$ 3.050$ & 0.0 & 0.0 & 0.0 & 0.0 & 0.0 & 0.0 & 0.0 & 0.0 & 0.0 & 0.0 \\
\hline & $\begin{array}{l}\text { Refrig. \& Thermal Dist. R\&D: Res. HVAC } \\
\text { Dist. System }\end{array}$ & $\$ 0.000$ & 0.8 & 2.1 & 3.8 & 6.3 & 9.7 & 27.9 & 66.5 & 61.5 & 28.5 & 18.4 \\
\hline & $\begin{array}{l}\text { Refrig. \& Thermal Dist. R\&D: Adv. Elec } \\
\text { HPWH }\end{array}$ & $\$ 0.000$ & 0.0 & 0.0 & 0.0 & 0.0 & 0.0 & 0.0 & 0.0 & 0.0 & 0.0 & 0.0 \\
\hline
\end{tabular}

\footnotetext{
Run Title: FY 2003 Run 4 NAS

Scenario Last Executed: $\quad$ 12/14/01

$1: 24: 50$ PM 
Primary Non-Electric Energy Savings (TBtu/yr)

\begin{tabular}{|c|c|c|c|c|c|c|c|c|c|c|c|}
\hline & $\begin{array}{c}\text { FY } 2003 \\
\text { Budget } \\
\text { (Million \$) }\end{array}$ & 2003 & 2004 & 2005 & 2006 & 2007 & 2010 & 2015 & 2020 & 2025 & 2030 \\
\hline $\begin{array}{l}\text { Refrig. \& Thermal Dist. R\&D: Commercial } \\
\text { Refrigeration }\end{array}$ & $\$ 0.000$ & 0.1 & 0.2 & 0.3 & 0.5 & 0.6 & 1.3 & 2.1 & 1.7 & 0.8 & 0.2 \\
\hline $\begin{array}{l}\text { Refrig. \& Thermal Dist. R\&D: Refrigerant } \\
\text { Meter }\end{array}$ & $\$ 0.000$ & 0.0 & 0.0 & 0.0 & 0.0 & 0.0 & 0.0 & 0.0 & 0.0 & 0.0 & 0.0 \\
\hline Emerging Technologies R\&D & $\$ 1.930$ & 0.0 & 0.0 & 0.0 & 0.0 & 0.0 & 0.0 & 0.0 & 0.0 & 0.0 & 0.0 \\
\hline Emerging Tech R\&D: HPWH & $\$ 0.000$ & 0.0 & 0.0 & 0.0 & 0.0 & 0.0 & 0.0 & 0.0 & 0.0 & 0.0 & 0.0 \\
\hline Emerging Tech R\&D: Roof Top AC & $\$ 0.000$ & 0.0 & 0.0 & 0.0 & 0.0 & 0.0 & 0.0 & 0.0 & 0.0 & 0.0 & 0.0 \\
\hline Emerging Tech R\&D: Gas Condensing WH & $\$ 0.000$ & 0.0 & 0.0 & 0.2 & 0.6 & 1.2 & 7.0 & 21.0 & 25.3 & 30.1 & 35.2 \\
\hline Emerging Tech R\&D: Recessed Can Lights & $\$ 0.000$ & 0.0 & 0.0 & 0.0 & 0.0 & 0.0 & 0.0 & -0.1 & -0.1 & -0.1 & -0.1 \\
\hline Emerging Tech R\&D: R-Lamp & $\$ 0.000$ & -0.1 & -0.1 & -0.3 & -0.5 & -0.6 & -1.0 & -0.2 & 0.0 & 0.0 & 0.0 \\
\hline Bldg. Env. R\&D: Windows & $\$ 3.630$ & 0.0 & 0.0 & 0.0 & 0.0 & 0.0 & 0.0 & 0.0 & 0.0 & 0.0 & 0.0 \\
\hline Envelope R\&D: Electrochromic Windows & $\$ 0.000$ & -0.7 & -1.0 & -1.4 & -1.7 & -2.0 & -2.8 & -3.4 & -3.4 & -3.4 & -3.3 \\
\hline Envelope R\&D: Superwindows & $\$ 0.000$ & 1.7 & 3.8 & 6.6 & 9.9 & 14.1 & 32.7 & 85.3 & 134.4 & 186.3 & 241.1 \\
\hline Bldg. Env. R\&D: Roofs and Insulation & $\$ 1.560$ & 0.0 & 0.0 & 0.0 & 0.0 & 0.0 & 0.0 & 0.0 & 0.0 & 0.0 & 0.0 \\
\hline Envelope R\&D: Quick Fill Walls & $\$ 0.000$ & 0.0 & 0.0 & 0.1 & 0.1 & 0.1 & 0.4 & 1.8 & 4.2 & 6.7 & 9.5 \\
\hline $\begin{array}{l}\text { Envelope R\&D: R30 Insulation/30 Year } \\
\text { Life Roofs }\end{array}$ & $\$ 0.000$ & 0.0 & 0.0 & 0.0 & 0.0 & 0.0 & 0.3 & 3.8 & 12.8 & 21.9 & 31.2 \\
\hline Envelope R\&D: Moisture/Wet Insulation & $\$ 0.000$ & 0.0 & 0.2 & 0.8 & 1.6 & 2.7 & 7.7 & 19.8 & 37.1 & 56.3 & 77.2 \\
\hline Design Strategies and Assistance & $\$ 3.130$ & 0.1 & 0.4 & 0.7 & 1.1 & 1.6 & 3.9 & 9.6 & 14.2 & 19.7 & 25.1 \\
\hline Standards & $\$ 8.930$ & 0.0 & 0.0 & 0.0 & 0.0 & 0.0 & 0.0 & 0.0 & 0.0 & 0.0 & 0.0 \\
\hline Standards: Res Gas Furnaces/Boilers & $\$ 0.000$ & 0.0 & 0.0 & 0.0 & 0.0 & 0.0 & 4.7 & 17.5 & 31.0 & 45.9 & 62.4 \\
\hline Standards: EPACT Standards & $\$ 0.000$ & 0.0 & 0.0 & 0.0 & 0.0 & 0.0 & 2.9 & 7.9 & 13.0 & 17.3 & 21.6 \\
\hline Standards: Dist. Transformers & $\$ 0.000$ & 0.0 & 0.0 & 0.0 & 0.0 & 0.0 & 0.0 & 0.0 & 0.0 & 0.0 & 0.0 \\
\hline Building Research and Standards (DU 5-7) & $\$ 47.940$ & 2.4 & 6.4 & 12.7 & 21.3 & 32.7 & 104.4 & 295.0 & 430.0 & 540.3 & 681.2 \\
\hline All BTS & $\$ 390.030$ & 23.6 & 45.9 & 71.3 & 91.7 & 114.6 & 224.1 & 474.1 & 644.0 & 778.9 & 944.9 \\
\hline
\end{tabular}

$\begin{array}{llc}\text { Run Title: } & \text { FY 2003 Run 4 NAS } & \text { Scenario Last Executed: } \\ \text { MDB: } & \text { K:IMETRICSIFY2003|BESET InputsIRun 4 NASIFY 2003 Run } 4 \text { NAS.mdb } & \text { 1:24:50 PM }\end{array}$




\section{SO2 Emissions Reductions}


$\mathrm{SO}_{2}$ Emissions Reductions (MMTons/yr)

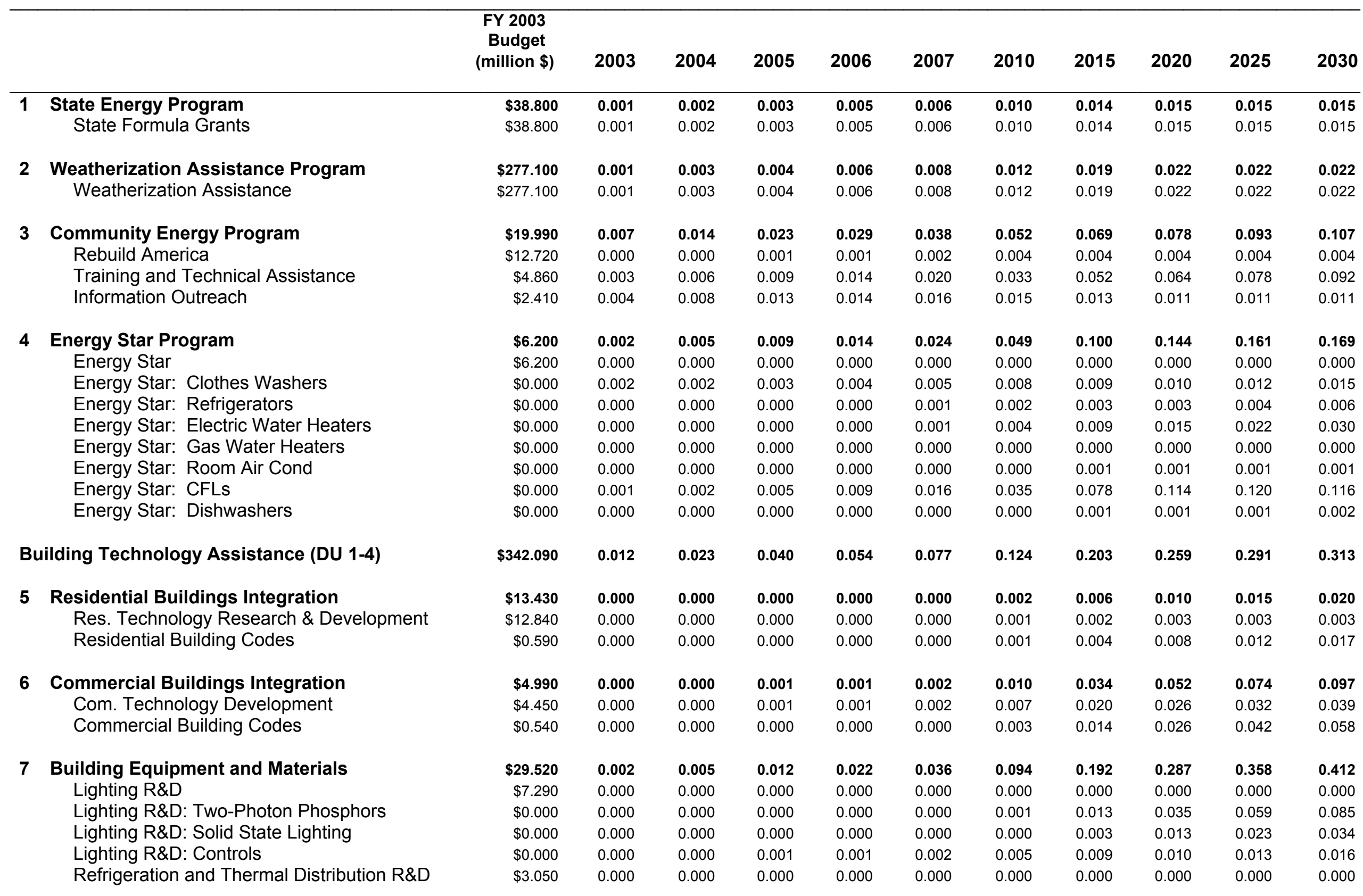

Run Title: FY 2003 Run 4 NAS

MDB: K:IMETRICSIFY2003IBESET InputsIRun 4 NASIFY 2003 Run 4 NAS.mdb

Scenario Last Executed: $12 / 14 / 01 \quad 1: 24: 50$ PM 
$\mathrm{SO}_{2}$ Emissions Reductions (MMTons/yr)

\begin{tabular}{|c|c|c|c|c|c|c|c|c|c|c|c|}
\hline & $\begin{array}{c}\text { FY } 2003 \\
\text { Budget } \\
\text { (million \$) }\end{array}$ & 2003 & 2004 & 2005 & 2006 & 2007 & 2010 & 2015 & 2020 & 2025 & 2030 \\
\hline $\begin{array}{l}\text { Refrig. \& Thermal Dist. R\&D: Res. HVAC } \\
\text { Dist. System }\end{array}$ & $\$ 0.000$ & 0.000 & 0.000 & 0.001 & 0.001 & 0.003 & 0.007 & 0.014 & 0.012 & 0.006 & 0.005 \\
\hline $\begin{array}{l}\text { Refrig. \& Thermal Dist. R\&D: Adv. Elec } \\
\text { HPWH }\end{array}$ & $\$ 0.000$ & 0.000 & 0.000 & 0.000 & 0.000 & 0.000 & 0.001 & 0.006 & 0.012 & 0.015 & 0.009 \\
\hline $\begin{array}{l}\text { Refrig. \& Thermal Dist. R\&D: Commercial } \\
\text { Refrigeration }\end{array}$ & $\$ 0.000$ & 0.000 & 0.001 & 0.001 & 0.002 & 0.003 & 0.006 & 0.008 & 0.005 & 0.002 & 0.001 \\
\hline $\begin{array}{l}\text { Refrig. \& Thermal Dist. R\&D: Refrigerant } \\
\text { Meter }\end{array}$ & $\$ 0.000$ & 0.000 & 0.000 & 0.000 & 0.000 & 0.001 & 0.003 & 0.015 & 0.031 & 0.035 & 0.020 \\
\hline Emerging Technologies R\&D & $\$ 1.930$ & 0.000 & 0.000 & 0.000 & 0.000 & 0.000 & 0.000 & 0.000 & 0.000 & 0.000 & 0.000 \\
\hline Emerging Tech R\&D: HPWH & $\$ 0.000$ & 0.000 & 0.000 & 0.001 & 0.001 & 0.003 & 0.008 & 0.016 & 0.017 & 0.021 & 0.025 \\
\hline Emerging Tech R\&D: Roof Top AC & $\$ 0.000$ & 0.000 & 0.000 & 0.000 & 0.000 & 0.000 & 0.001 & 0.001 & 0.001 & 0.001 & 0.001 \\
\hline Emerging Tech R\&D: Gas Condensing WH & $\$ 0.000$ & 0.000 & 0.000 & 0.000 & 0.000 & 0.000 & 0.000 & 0.000 & 0.000 & 0.000 & 0.000 \\
\hline Emerging Tech R\&D: Recessed Can Lights & $\$ 0.000$ & 0.000 & 0.000 & 0.000 & 0.000 & 0.000 & 0.001 & 0.001 & 0.001 & 0.001 & 0.001 \\
\hline Emerging Tech R\&D: R-Lamp & $\$ 0.000$ & 0.000 & 0.001 & 0.002 & 0.004 & 0.007 & 0.010 & 0.001 & 0.000 & 0.000 & 0.000 \\
\hline Envelope R\&D: Electrochromic Windows & $\$ 0.000$ & 0.001 & 0.001 & 0.002 & 0.003 & 0.005 & 0.008 & 0.012 & 0.015 & 0.021 & 0.028 \\
\hline Envelope R\&D: Superwindows & $\$ 0.000$ & 0.000 & 0.001 & 0.002 & 0.003 & 0.004 & 0.009 & 0.021 & 0.030 & 0.041 & 0.053 \\
\hline Bldg. Env. R\&D: Roofs and Insulation & $\$ 1.560$ & 0.000 & 0.000 & 0.000 & 0.000 & 0.000 & 0.000 & 0.000 & 0.000 & 0.000 & 0.000 \\
\hline Envelope R\&D: Quick Fill Walls & $\$ 0.000$ & 0.000 & 0.000 & 0.000 & 0.000 & 0.000 & 0.000 & 0.000 & 0.000 & 0.001 & 0.001 \\
\hline $\begin{array}{l}\text { Envelope R\&D: R30 Insulation/30 Year } \\
\text { Life Roofs }\end{array}$ & $\$ 0.000$ & 0.000 & 0.000 & 0.000 & 0.000 & 0.000 & 0.000 & 0.000 & 0.001 & 0.002 & 0.002 \\
\hline Envelope R\&D: Moisture/Wet Insulation & $\$ 0.000$ & 0.000 & 0.000 & 0.000 & 0.000 & 0.000 & 0.000 & 0.001 & 0.002 & 0.003 & 0.005 \\
\hline Design Strategies and Assistance & $\$ 3.130$ & 0.000 & 0.000 & 0.001 & 0.001 & 0.002 & 0.004 & 0.008 & 0.011 & 0.015 & 0.019 \\
\hline Standards & $\$ 8.930$ & 0.000 & 0.000 & 0.000 & 0.000 & 0.000 & 0.000 & 0.000 & 0.000 & 0.000 & 0.000 \\
\hline Standards: Res Gas Furnaces/Boilers & $\$ 0.000$ & 0.000 & 0.000 & 0.000 & 0.000 & 0.000 & 0.000 & 0.000 & 0.000 & 0.000 & 0.000 \\
\hline Standards: EPACT Standards & $\$ 0.000$ & 0.000 & 0.000 & 0.002 & 0.004 & 0.007 & 0.025 & 0.051 & 0.072 & 0.081 & 0.090 \\
\hline Standards: Dist. Transformers & $\$ 0.000$ & 0.000 & 0.000 & 0.000 & 0.000 & 0.000 & 0.006 & 0.013 & 0.018 & 0.018 & 0.018 \\
\hline Building Research and Standards (DU 5-7) & $\$ 47.940$ & 0.002 & 0.006 & 0.013 & 0.024 & 0.039 & 0.106 & 0.232 & 0.349 & 0.448 & 0.529 \\
\hline All BTS & $\$ 390.030$ & 0.015 & 0.029 & 0.053 & 0.078 & 0.116 & 0.229 & 0.435 & 0.608 & 0.739 & 0.843 \\
\hline
\end{tabular}

Run Title: FY 2003 Run 4 NAS

MDB: $\quad$ K:IMETRICSIFY2003IBESET InputsIRun 4 NASIFY 2003 Run 4 NAS.mdb

Scenario Last Executed: 12/14/01 1:24:50 PM 


\section{VOC Emissions Reductions}


VOC Emissions Reductions (MMTons/yr)

\begin{tabular}{|c|c|c|c|c|c|c|c|c|c|c|c|c|}
\hline & & $\begin{array}{r}\text { FY } 2003 \\
\text { Budget } \\
\text { (mllion \$) }\end{array}$ & 2003 & 2004 & 2005 & 2006 & 2007 & 2010 & 2015 & 2020 & 2025 & 2030 \\
\hline \multirow[t]{2}{*}{1} & State Energy Program & $\$ 38.800$ & 0.000 & 0.000 & 0.000 & 0.000 & 0.000 & 0.000 & 0.000 & 0.000 & 0.000 & 0.000 \\
\hline & State Formula Grants & $\$ 38.800$ & 0.000 & 0.000 & 0.000 & 0.000 & 0.000 & 0.000 & 0.000 & 0.000 & 0.000 & 0.000 \\
\hline \multirow[t]{2}{*}{2} & Weatherization Assistance Program & $\$ 277.100$ & 0.000 & 0.000 & 0.000 & 0.000 & 0.000 & 0.000 & 0.000 & 0.000 & 0.000 & 0.000 \\
\hline & Weatherization Assistance & $\$ 277.100$ & 0.000 & 0.000 & 0.000 & 0.000 & 0.000 & 0.000 & 0.000 & 0.000 & 0.000 & 0.000 \\
\hline \multirow[t]{4}{*}{3} & Community Energy Program & $\$ 19.990$ & 0.000 & 0.000 & 0.000 & 0.000 & 0.000 & 0.000 & 0.001 & 0.001 & 0.001 & 0.001 \\
\hline & Rebuild America & $\$ 12.720$ & 0.000 & 0.000 & 0.000 & 0.000 & 0.000 & 0.000 & 0.000 & 0.000 & 0.000 & 0.000 \\
\hline & Training and Technical Assistance & $\$ 4.860$ & 0.000 & 0.000 & 0.000 & 0.000 & 0.000 & 0.000 & 0.000 & 0.001 & 0.001 & 0.001 \\
\hline & Information Outreach & $\$ 2.410$ & 0.000 & 0.000 & 0.000 & 0.000 & 0.000 & 0.000 & 0.000 & 0.000 & 0.000 & 0.000 \\
\hline \multirow[t]{9}{*}{4} & Energy Star Program & $\$ 6.200$ & 0.000 & 0.000 & 0.000 & 0.000 & 0.000 & 0.000 & 0.001 & 0.001 & 0.001 & 0.002 \\
\hline & Energy Star & $\$ 6.200$ & 0.000 & 0.000 & 0.000 & 0.000 & 0.000 & 0.000 & 0.000 & 0.000 & 0.000 & 0.000 \\
\hline & Energy Star: Clothes Washers & $\$ 0.000$ & 0.000 & 0.000 & 0.000 & 0.000 & 0.000 & 0.000 & 0.000 & 0.000 & 0.000 & 0.000 \\
\hline & Energy Star: Refrigerators & $\$ 0.000$ & 0.000 & 0.000 & 0.000 & 0.000 & 0.000 & 0.000 & 0.000 & 0.000 & 0.000 & 0.000 \\
\hline & Energy Star: Electric Water Heaters & $\$ 0.000$ & 0.000 & 0.000 & 0.000 & 0.000 & 0.000 & 0.000 & 0.000 & 0.000 & 0.000 & 0.000 \\
\hline & Energy Star: Gas Water Heaters & $\$ 0.000$ & 0.000 & 0.000 & 0.000 & 0.000 & 0.000 & 0.000 & 0.000 & 0.000 & 0.000 & 0.000 \\
\hline & Energy Star: Room Air Cond & $\$ 0.000$ & 0.000 & 0.000 & 0.000 & 0.000 & 0.000 & 0.000 & 0.000 & 0.000 & 0.000 & 0.000 \\
\hline & Energy Star: CFLs & $\$ 0.000$ & 0.000 & 0.000 & 0.000 & 0.000 & 0.000 & 0.000 & 0.001 & 0.001 & 0.001 & 0.001 \\
\hline & Energy Star: Dishwashers & $\$ 0.000$ & 0.000 & 0.000 & 0.000 & 0.000 & 0.000 & 0.000 & 0.000 & 0.000 & 0.000 & 0.000 \\
\hline \multicolumn{2}{|r|}{ Building Technology Assistance (DU 1-4) } & $\$ 342.090$ & 0.000 & 0.000 & 0.000 & 0.001 & 0.001 & 0.001 & 0.002 & 0.003 & 0.003 & 0.003 \\
\hline \multirow[t]{3}{*}{5} & Residential Buildings Integration & $\$ 13.430$ & 0.000 & 0.000 & 0.000 & 0.000 & 0.000 & 0.000 & 0.000 & 0.000 & 0.000 & 0.000 \\
\hline & Res. Technology Research \& Development & $\$ 12.840$ & 0.000 & 0.000 & 0.000 & 0.000 & 0.000 & 0.000 & 0.000 & 0.000 & 0.000 & 0.000 \\
\hline & Residential Building Codes & $\$ 0.590$ & 0.000 & 0.000 & 0.000 & 0.000 & 0.000 & 0.000 & 0.000 & 0.000 & 0.000 & 0.000 \\
\hline \multirow[t]{3}{*}{6} & Commercial Buildings Integration & $\$ 4.990$ & 0.000 & 0.000 & 0.000 & 0.000 & 0.000 & 0.000 & 0.000 & 0.001 & 0.001 & 0.001 \\
\hline & Com. Technology Development & $\$ 4.450$ & 0.000 & 0.000 & 0.000 & 0.000 & 0.000 & 0.000 & 0.000 & 0.000 & 0.000 & 0.001 \\
\hline & Commercial Building Codes & $\$ 0.540$ & 0.000 & 0.000 & 0.000 & 0.000 & 0.000 & 0.000 & 0.000 & 0.000 & 0.000 & 0.000 \\
\hline \multirow[t]{6}{*}{7} & Building Equipment and Materials & $\$ 29.520$ & 0.000 & 0.000 & 0.000 & 0.000 & 0.000 & 0.001 & 0.002 & 0.003 & 0.004 & 0.005 \\
\hline & Lighting R\&D & $\$ 7.290$ & 0.000 & 0.000 & 0.000 & 0.000 & 0.000 & 0.000 & 0.000 & 0.000 & 0.000 & 0.000 \\
\hline & Lighting R\&D: Two-Photon Phosphors & $\$ 0.000$ & 0.000 & 0.000 & 0.000 & 0.000 & 0.000 & 0.000 & 0.000 & 0.000 & 0.000 & 0.001 \\
\hline & Lighting R\&D: Solid State Lighting & $\$ 0.000$ & 0.000 & 0.000 & 0.000 & 0.000 & 0.000 & 0.000 & 0.000 & 0.000 & 0.000 & 0.000 \\
\hline & Lighting R\&D: Controls & $\$ 0.000$ & 0.000 & 0.000 & 0.000 & 0.000 & 0.000 & 0.000 & 0.000 & 0.000 & 0.000 & 0.000 \\
\hline & Refrigeration and Thermal Distribution R\&D & $\$ 3.050$ & 0.000 & 0.000 & 0.000 & 0.000 & 0.000 & 0.000 & 0.000 & 0.000 & 0.000 & 0.000 \\
\hline
\end{tabular}

Run Title: FY 2003 Run 4 NAS

MDB: $\quad$ K:IMETRICSIFY2003IBESET InputsIRun 4 NASIFY 2003 Run 4 NAS.mdb

Scenario Last Executed: $\quad 12 / 14 / 01 \quad 1: 24: 50$ PM 
VOC Emissions Reductions (MMTons/yr)

\begin{tabular}{|c|c|c|c|c|c|c|c|c|c|c|c|}
\hline & $\begin{array}{c}\text { FY } 2003 \\
\text { Budget } \\
\text { (mllion \$) }\end{array}$ & 2003 & 2004 & 2005 & 2006 & 2007 & 2010 & 2015 & 2020 & 2025 & 2030 \\
\hline $\begin{array}{l}\text { Refrig. \& Thermal Dist. R\&D: Res. HVAC } \\
\text { Dist. System }\end{array}$ & $\$ 0.000$ & 0.000 & 0.000 & 0.000 & 0.000 & 0.000 & 0.000 & 0.000 & 0.000 & 0.000 & 0.000 \\
\hline $\begin{array}{l}\text { Refrig. \& Thermal Dist. R\&D: Adv. Elec } \\
\text { HPWH }\end{array}$ & $\$ 0.000$ & 0.000 & 0.000 & 0.000 & 0.000 & 0.000 & 0.000 & 0.000 & 0.000 & 0.000 & 0.000 \\
\hline $\begin{array}{l}\text { Refrig. \& Thermal Dist. R\&D: Commercial } \\
\text { Refrigeration }\end{array}$ & $\$ 0.000$ & 0.000 & 0.000 & 0.000 & 0.000 & 0.000 & 0.000 & 0.000 & 0.000 & 0.000 & 0.000 \\
\hline $\begin{array}{l}\text { Refrig. \& Thermal Dist. R\&D: Refrigerant } \\
\text { Meter }\end{array}$ & $\$ 0.000$ & 0.000 & 0.000 & 0.000 & 0.000 & 0.000 & 0.000 & 0.000 & 0.000 & 0.000 & 0.000 \\
\hline Emerging Technologies R\&D & $\$ 1.930$ & 0.000 & 0.000 & 0.000 & 0.000 & 0.000 & 0.000 & 0.000 & 0.000 & 0.000 & 0.000 \\
\hline Emerging Tech R\&D: HPWH & $\$ 0.000$ & 0.000 & 0.000 & 0.000 & 0.000 & 0.000 & 0.000 & 0.000 & 0.000 & 0.000 & 0.000 \\
\hline Emerging Tech R\&D: Roof Top AC & $\$ 0.000$ & 0.000 & 0.000 & 0.000 & 0.000 & 0.000 & 0.000 & 0.000 & 0.000 & 0.000 & 0.000 \\
\hline Emerging Tech R\&D: Gas Condensing WH & $\$ 0.000$ & 0.000 & 0.000 & 0.000 & 0.000 & 0.000 & 0.000 & 0.000 & 0.000 & 0.000 & 0.000 \\
\hline Emerging Tech R\&D: Recessed Can Lights & $\$ 0.000$ & 0.000 & 0.000 & 0.000 & 0.000 & 0.000 & 0.000 & 0.000 & 0.000 & 0.000 & 0.000 \\
\hline Emerging Tech R\&D: R-Lamp & $\$ 0.000$ & 0.000 & 0.000 & 0.000 & 0.000 & 0.000 & 0.000 & 0.000 & 0.000 & 0.000 & 0.000 \\
\hline Envelope R\&D: Electrochromic Windows & $\$ 0.000$ & 0.000 & 0.000 & 0.000 & 0.000 & 0.000 & 0.000 & 0.000 & 0.000 & 0.000 & 0.000 \\
\hline Envelope R\&D: Superwindows & $\$ 0.000$ & 0.000 & 0.000 & 0.000 & 0.000 & 0.000 & 0.000 & 0.000 & 0.001 & 0.001 & 0.001 \\
\hline Bldg. Env. R\&D: Roofs and Insulation & $\$ 1.560$ & 0.000 & 0.000 & 0.000 & 0.000 & 0.000 & 0.000 & 0.000 & 0.000 & 0.000 & 0.000 \\
\hline Envelope R\&D: Quick Fill Walls & $\$ 0.000$ & 0.000 & 0.000 & 0.000 & 0.000 & 0.000 & 0.000 & 0.000 & 0.000 & 0.000 & 0.000 \\
\hline $\begin{array}{l}\text { Envelope R\&D: R30 Insulation/30 Year } \\
\text { Life Roofs }\end{array}$ & $\$ 0.000$ & 0.000 & 0.000 & 0.000 & 0.000 & 0.000 & 0.000 & 0.000 & 0.000 & 0.000 & 0.000 \\
\hline Envelope R\&D: Moisture/Wet Insulation & $\$ 0.000$ & 0.000 & 0.000 & 0.000 & 0.000 & 0.000 & 0.000 & 0.000 & 0.000 & 0.000 & 0.000 \\
\hline Design Strategies and Assistance & $\$ 3.130$ & 0.000 & 0.000 & 0.000 & 0.000 & 0.000 & 0.000 & 0.000 & 0.000 & 0.000 & 0.000 \\
\hline Standards & $\$ 8.930$ & 0.000 & 0.000 & 0.000 & 0.000 & 0.000 & 0.000 & 0.000 & 0.000 & 0.000 & 0.000 \\
\hline Standards: Res Gas Furnaces/Boilers & $\$ 0.000$ & 0.000 & 0.000 & 0.000 & 0.000 & 0.000 & 0.000 & 0.000 & 0.000 & 0.000 & 0.000 \\
\hline Standards: EPACT Standards & $\$ 0.000$ & 0.000 & 0.000 & 0.000 & 0.000 & 0.000 & 0.000 & 0.000 & 0.001 & 0.001 & 0.001 \\
\hline Standards: Dist. Transformers & $\$ 0.000$ & 0.000 & 0.000 & 0.000 & 0.000 & 0.000 & 0.000 & 0.000 & 0.000 & 0.000 & 0.000 \\
\hline Building Research and Standards (DU 5-7) & $\$ 47.940$ & 0.000 & 0.000 & 0.000 & 0.000 & 0.000 & 0.001 & 0.002 & 0.004 & 0.005 & 0.006 \\
\hline All BTS & $\$ 390.030$ & 0.000 & 0.000 & 0.001 & 0.001 & 0.001 & 0.002 & 0.004 & 0.007 & 0.008 & 0.009 \\
\hline
\end{tabular}

Run Title: FY 2003 Run 4 NAS

Scenario Last Executed: $12 / 14 / 01 \quad 1: 24: 50$ PM

MDB: $\quad$ K:IMETRICSIFY2003IBESET InputsIRun 4 NASIFY 2003 Run 4 NAS.mdb 
State Energy 


\section{Program Objective:(1)}

The State Energy Program (SEP) provides a supportive framework with sufficient flexibility to enable states to address their energy priorities in concert with national priorities. It supports the federal/state partnerships that are crucial to developing energy policies and deploying energy technologies. SEP emphasizes outreach, technology deployment, and the forming of partnerships to accomplish energy efficiency and renewable energy projects at the state and local level.

\section{Long-Term Goal:(1)}

The program's strategic plan for the $21^{\text {st }}$ century establishes three key goals to be accomplished by 2010 :

1) maximize energy, environmental, and economic benefits through increased collaboration at the federal, state and community level; 2) increase market acceptance of energy efficiency and renewable energy technologies, practices, and products; and 3) use innovative approaches to reach market segments and meet policy goals not typically addressed by market-based solutions.

\section{Market Segment:}

Target Market

- Market Description: All markets (including buildings, transportation, industry, and power technologies) except new construction and all categories of energy end use.

\section{Methodology}

For the GPRA metrics, the State Formula Grants program is characterized based on an estimated level of savings per budget dollar, budget request, and leveraged funds. The basic assumptions were derived from a spreadsheet provided by the program in FY 1999. The assumptions were revised slightly because of external peer review by A.D. Little for the FY 2002 effort. Because this is a grant program, this activity was assumed not to occur without DOE funding; therefore, the NAS (acceleration-to-market) methodology was not applied.

\section{Estimated Savings Per Budget Dollar:}

For FY 2001, each program dollar was assumed to yield 0.0063 MBtu of delivered electricity savings and 0.012

\section{State Formula Grants}

Program Type:

Grant

Target Market:

All sectors in all climate zones

End Uses:

All end uses, all fuel types

Unit of Measurement:

Energy savings per budget dollar

Modeling Tool:

Spreadsheet

Program Manager:

Faith Lambert

Website:

http://www.eren.doe.gov/

buildings/state_energy/

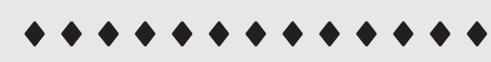

FY 2003 Benefits

Primary Energy Savings (TBtu)

\begin{tabular}{llll}
2003 & 2005 & 2010 & 2020 \\
\hline 3.9 & 11.5 & 27.7 & 47.7
\end{tabular}

Carbon Equivalent Reductions (MMTCE)

\begin{tabular}{cccc}
2003 & 2005 & 2010 & 2020 \\
\hline .070 & .216 & .549 & .913
\end{tabular}

Consumer Cost Savings (million \$)

\begin{tabular}{llll}
2003 & 2005 & 2010 & 2020 \\
\hline 24 & 70 & 177 & 345
\end{tabular}




\section{State Formula Grants}

MBtu of other fuel savings per year. These figures were based on an historical review of SEP savings for 1987-1993, as provided in the SEP spreadsheet. To develop energy savings by fuel type for other fuel savings, a split of $75 \%$ natural gas and $25 \%$ fuel oil was assumed.

While this same level of savings was used initially for FY 2002, it was modified based on the A.D. Little peer review. Based on concerns about the historical versus future project mix, savings based on recyding and wood and biomass renewables were removed.

These revisions resulted in an estimate of $0.0044 \mathrm{MBtu}$ of delivered electric savings and $0.0065 \mathrm{MBtu}$ of other fuel savings per program dollar per year. For FY 2002, the fuel split was modified to reflect historical information, yielding a split of $20 \%$ natural gas and $80 \%$ fuel oil. The savings also were split between the residential and commercial sectors (with the commercial sector representing savings from industrial, transportation, and utilities) based on the historical split of savings (7\% residential and $93 \%$ nonresidential).

For FY 2003, the revised estimates developed in FY 2002 were used.
Budget Request and Leveraged Funding: The estimated energy savings were calculated using the FY 2003 budget request, the budget forecast for FY 2004 - FY 2030, and a leveraged funding forecast. Funds were assumed to be leveraged at a ratio of $\$ 4$ for every budget dollar.

\section{Non-Energy Benefits:}

Cleaner air and water, increased jobs, enhanced national security, increased economic competitiveness in world markets, and mitigation of global warming.(1)

\section{Program Strategy (\% of budget):(1)}

- Research and Development - 0\%

- Market Transformation - 95\%

- Codes and Standards - 5\%.

\section{Sources:}

(1) FY 2002 Budget Request - Data Bucket Report for State Formula Grants Program (internal BTS document). 


\section{Weatherization Assistance}




\section{Weatherization Assistance Program}

Weatherization Assistance Program Decision Unit

\section{Program Objective:(1)}

The Weatherization Assistance Program provides cost-effective energy-efficiency services to low-income constituencies who otherwise could not afford the investment but who would benefit significantly from the cost savings of energy-efficiency technologies. The program focuses on households that spend a disproportionate amount of their income for energy, giving priority to households with elderly members, persons with disabilities, and children.

In 1999 the Weatherization network of state and local agencies adopted a new strategic vision for the program called Weatherization Plus. The new strategy emphasizes a shift to the whole-house approach and includes electric baseload measures and advanced technologies. Within the new $\$ 2500$ legislative cap on average expenditure per household, the mix of measures include those with enhanced impacts on greenhouse gas emissions and pollution reduction. Such measures indude intensified building envelope and heating/cooling system measures, more health and safety measures (supporting other community goals), and more baseload uses such as water heating and lighting.

\section{Long-Term Goal:(1)}

The long-term goal of the Weatherization Assistance Program is to achieve average energy savings of $31 \mathrm{Mbtu}$ in an additional 2.3 million existing low-income homes through 2011, consistent with the President's plan to increase Weatherization funding by $\$ 1.4$ billion over FY 2000 baselines for the 10-year period. This investment will save participating households an estimated $\$ 4.2$ billion in reduced energy bills over the period.

\section{Market Segment:}

\section{Target Market}

- Market Description: Low-income homes and target measures include air sealing; caulking and weather stripping; furnace and boiler tuneup, repair, and replacement; cooling system tuneup and repair; replacement of windows and doors; addition of storm windows and doors; insulation of building shells; and replacement of air conditioners, wholehouse fans, evaporative coolers, screening, and win-

\section{Weatherization Assistance Program}

Program Type:

Envelope/Grant

Target Market:

Low-income residential housing in

all climate zones

End Uses:

All end uses, all fuel types

Unit of Measurement:

Energy savings per housing unit

Modeling Tool:

Spreadsheet

Program Manager:

Greg Reamy

Website:

http://www.eren.doe.gov/buildings/ weatherization_assistance/

FY 2003 Benefits

Primary Energy Savings (TBtu)

\begin{tabular}{llll}
2003 & 2005 & 2010 & 2020 \\
\hline 8.4 & 25.3 & 65.7 & 122.5
\end{tabular}

Carbon Equivalent Reductions (MMTCE)

\begin{tabular}{cccc}
2003 & 2005 & 2010 & 2020 \\
\hline 139 & .422 & 1.114 & 2.040
\end{tabular}

Consumer Cost Savings (million \$)

\begin{tabular}{lllr}
2003 & 2005 & 2010 & 2020 \\
\hline 59 & 175 & 469 & 917
\end{tabular}


Weatherization Assistance Program Decision Unit

dow films. ${ }^{(2)}$ Weatherization Plus would expand this strategy to include water heating, refrigeration, lighting, and cooling.(1)

- Size of Market: About 29 million eligible low-income homes.

\section{Methodology}

For the GPRA metrics, this program was characterized based on an estimated level of savings per household, cost to weatherize each household, budget request, leveraged funds, and an assumed life expectancy of 15 years for weatherization measures. The basic assumptions were derived from a weatherization spreadsheet provided by the Weatherization program in September 2001.

\section{Estimated Savings Per Household:}

For the FY 2002 and FY 2003 metrics, the savings per household used for each region are shown in the table below.

\begin{tabular}{|l|c|c|}
\hline \multicolumn{1}{|c|}{ Region } & $\begin{array}{c}\text { Regular Household } \\
\text { Savings } \\
\text { (MMBtu/yr) }\end{array}$ & $\begin{array}{c}\text { Plus Household } \\
\text { Savings } \\
\text { (MMBtu/yr) }\end{array}$ \\
\hline South & 22.25 & 24.23 \\
\hline Northeast & 31.20 & 46.04 \\
\hline West & 19.04 & 20.31 \\
\hline Midwest & 31.20 & 49.21 \\
\hline
\end{tabular}

The table's figures were calculated based on the 1997 ORNL Metaevaluation report, (2) the ORNL Meeting the Challenge report, ${ }^{(4)}$ and special tabulations from the 1997 RECS. ${ }^{(5)}$ Previous year's estimates were based on program re source allocations at levels reflecting a formula bias towards homes in colder dimates in the Northeast and Midwest. The higher appropriation levels projected for FY 2002 and beyond will be allocated under a formula that shifts a higher proportion of new revenues to the South and West, where saving rates are lower.

Of the units weatherized in FY 2002, 20\% were assumed to have the higher savings rates associated with Weatherization Plus.
In Meeting The Challenge, ${ }^{(4)}$ these savings rates were calculated on a regional basis and multiplied times the expected number of Plus households in each region. For FY 2003, the proportion of Plus homes weatherized increased from $20 \%$ to $30 \%$ of the total.

To develop energy savings by fuel type, the fuel split was based on historical Weatherization program data in the 1997 ORNL report ${ }^{(2)}$ concerning the primary heating fuel of weatherized households. Because the GPRA metrics are reported only for electricity, natural gas, and fuel oil, the other fuel percentages were allocated within those types based on similarities of emissions. The split was allocated as shown in the table below.

\begin{tabular}{|l|c|l|}
\hline Primary Heating Fuel & $\begin{array}{c}\text { \% of } \\
\text { Weatherized } \\
\text { Households }\end{array}$ & $\begin{array}{c}\text { Categorized } \\
\text { As }\end{array}$ \\
\hline Natural Gas & 50.6 & Natural Gas \\
Liquid Propane Gas & 13.2 & \\
\hline Fuel Oil & 16.0 & Fuel Oil \\
Kerosene & 3.2 & \\
Other (includes wood & 7.5 & \\
and coal) & & \\
\hline Electricity & 9.5 & Electricity \\
\hline
\end{tabular}

Cost to Weatherize Each Household:

For FY 2002, the average cost of $\$ 1725$ to weatherize each household was used, not including training and technical assistance and administrative costs. ${ }^{(1)}$ For FY 2003, $\$ 1750$ was used for regular homes. Incremental investment for Weatherization Plus homes, estimated at an average of $\$ 1400$, was assumed to be derived from leveraged funds. Estimated costs by region for Plus homes are shown below.

\begin{tabular}{|l|c|}
\hline \multicolumn{1}{|c|}{ Region } & $\begin{array}{c}\text { Cost per Plus } \\
\text { Household }\end{array}$ \\
\hline South & $\$ 2861$ \\
\hline Northeast & $\$ 3674$ \\
\hline West & $\$ 1814$ \\
\hline Midwest & $\$ 3429$ \\
\hline
\end{tabular}




\section{Weatherization Assistance Program}

Weatherization Assistance Program Decision Unit

DOE Funds Available:

Because this is a grant program, this activity was assumed not to occur without DOE funding; therefore, the NAS methodology was not applied. The FY 2003 Weatherization planning budget and forecast for $F Y$ 2003 to FY 2030 was used to calculate the number of households weatherized. The total funds allocated to training and technical assistance and administrative costs were estimated to total $20 \%$ during the period.

\section{Leveraged Funding:}

For FY 2002, leveraged funding of $\$ 235$ million per year was assumed. A $20 \%$ program overhead was subtracted from the total before calculating the number of households weatherized with these funds. Leveraged funding for the DOE Plus homes was estimated to total $\$ 34.7$ million in FY 2002 and $\$ 52.5$ million in FY 2003, based on the costs reflected in ORNL's Meeting The Challenge report. (4) The balance of leveraged funds was assumed to be used on regular and Plus homes in a 70/30 ratio in FY 2002 and a 60/40 ratio in FY 2003.

\section{Non-Energy Benefits:}

A net present value of $\$ 161$ per household(5) (1989 \$), adjusted for inflation, was used for the FY 2003 effort, based on the estimated non-energy benefits resulting from enhanced property values and extended lifetimes of the dwellings, reduced fires, and reduced arrearages. Non-energy benefits associated with employment and environmental exter- nalities are captured elsewhere in reporting and therefore are not included here.

\section{Program Strategy (\% of budget):}

- Research and Development - 0\%

- Market Transformation - 100\%

- Codes and Standards - 0\%.

\section{Sources:}

(1) FY 2002 Budget Request - Data Bucket Report of Weatherization Assistance Program (internal BTS document).

(2) Berry, L.G., M.A. Brown, and L.F. Kinney. 1997. Progress Report of the National Weatherization Assistance Program. ORNL/CON-450, Oak Ridge National Laboratory, Oak Ridge, Tennessee.

(3) Eisenberg, J .F. Special tabulations for the Weatherization Population derived from the 1997 Residential Energy Consumption Survey. Oak Ridge National Laboratory, Oak Ridge, Tennessee.

(4) Schweitzer, M. and J .F. E isenberg. 2000. Meeting The Challenge: The Prospect of Achieving 30 Percent Energy Savings Through the Weatherization Assistance Program. ORNL/CON 479, Draft Analysis, Oak Ridge National Laboratory, Oak Ridge, Tennessee.

(5) Brown, M.A., L.G. Bery, R.A. Balzer, and E. Faby. 1993. National Impacts of the Weatherization Assistance Program in SingleFamily and Small Multifamily Dwellings. ORNL/CON-326, Oak Ridge National Laboratory, Oak Ridge, Tennessee. 


\section{Community Energy}




\section{Rebuild America}




\section{Rebuild America/Energy Smart Schools}

Community Energy Program Decision Unit

Rebuild America

\section{Program Objective:(1)}

This program builds collaborative partnerships with states and communities to help them develop and implement environmentally and economically sound activities through smarter energy use. Rebuild America connects people, resources, proven ideas, and innovative practices to solve problems. The program provides onestop shopping for information and assistance on how to plan, finance, implement, and manage retrofit projects to improve buildings' energy efficiency and helps communities find other resources on renewable energy applications, efficient new building designs, energy education, and other innovative energy conservation measures.

Rebuild America supports the public/private Energy Smart Schools initiative and competitive Community Energy Grants to encourage community-wide energy projects.

\section{Long-Term Goal:(1)}

By 2010, the program's long-term goals include the following: 2000 partnerships, 4 billion sq ft committed to retrofit ( $\sim 5 \%$ of the market, 2 million sq ft/partnership), $\$ 6$ billion in private investments committed, 0.2 quad of energy saved, and $\$ 1.3 \mathrm{billion} / \mathrm{yr}$ savings.

\section{Market Segment:}

\section{Performance Objective:(2)}

- Displaced Technology: Current design/building practices.

- Performance Target: Reduce heating, cooling, and water heat energy use in retrofitted and new buildings by $25 \% / \mathrm{sq} \mathrm{ft}$ in 2001 and $40 \% / \mathrm{sq} \mathrm{ft}$ by 2010 .

\section{Target Market ${ }^{(2)}$}

- Market Description: Rebuild America helps designated communities design and implement energysaving programs that respond to their own circumstances and goals, providing access to a portfolio of technical assistance, with a focus on existing commercial and institutional buildings. The general target market includes new and existing multifamily housing, public/assisted single-family residential units, and commercial buildings, particularly, new and existing assembly, health care, lodging, office, and education buildings.

\section{Rebuild America}

\section{Program Type:}

Whole Building

Target Market:

Existing multifamily residential units having homeowners with $>\$ 25,000 /$ $\mathrm{yr}$ income and the commercial sector in all climate zones

\section{End Uses:}

All end uses, all fuel types

Unit of Measurement:

$\%$ change in load

Modeling Tool:

BESET

Program Manager:

Daniel Sze

Website:

http://www.eren.doe.gov/buildings/ rebuild/

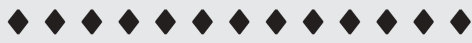

FY 2003 Benefits

Primary Energy Savings (TBtu)

\begin{tabular}{llll}
2003 & 2005 & 2010 & 2020 \\
\hline 0.7 & 5.3 & 21.7 & 25.8
\end{tabular}

Carbon Equivalent Reductions (MMTCE)

\begin{tabular}{cccc}
2003 & 2005 & 2010 & 2020 \\
\hline .011 & .089 & .380 & .438
\end{tabular}

Consumer Cost Savings (million \$)

\begin{tabular}{llll}
2003 & 2005 & 2010 & 2020 \\
\hline 5 & 34 & 148 & 193
\end{tabular}


- Market Size: See the following table.

\begin{tabular}{|l|c|c|}
\hline $\begin{array}{c}\text { Market Segments } \\
\text { and Sectors }\end{array}$ & $\begin{array}{c}\text { Floor Space } \\
\text { (billion sq ft) }\end{array}$ & $\begin{array}{c}\text { Energy Use/ } \\
\text { Yr (TBtu) }\end{array}$ \\
\hline Commercial Total & 67.9 & 5,800 \\
\hline $\begin{array}{l}\text { Commercial Local } \\
\text { and State }\end{array}$ & 16.5 & 1,480 \\
\hline Commercial Private & 51.4 & 4,320 \\
\hline Residential Total & 26.8 & 1,957 \\
\hline $\begin{array}{l}\text { Residential } \\
\text { Public/Assisted } \\
\text { Housing }\end{array}$ & 4.8 & 450 \\
\hline $\begin{array}{l}\text { Residential Private } \\
\text { Multifamily Housing }\end{array}$ & 22.0 & 1,507 \\
\hline $\begin{array}{l}\text { Total Commercial } \\
\text { and Residential }\end{array}$ & 94.7 & 7,757 \\
\hline
\end{tabular}

- Penetration Goal: This activity was assumed not to occur without DOE funding; therefore, the NAS methodology was not applied. The penetration goal is to have 2,000 partnerships retrofitting 2 million sq $\mathrm{ft}$ each by 2010 . The resulting penetration rates based are shown in the table below.

\begin{tabular}{|c|c|c|c|}
\hline \multirow{2}{*}{$\begin{array}{c}\text { Building } \\
\text { Type }^{*}\end{array}$} & \multicolumn{3}{|c|}{ Penetration Rates (\%) } \\
\hline & 2010 & 2020 & 2030 \\
\hline Assembly & 2.9 & 3.3 & 3.2 \\
\hline Education & 2.9 & 3.3 & 3.2 \\
\hline Health Care & 2.9 & 3.3 & 3.2 \\
\hline Lodging & 2.9 & 3.3 & 3.2 \\
\hline Office, Large & 2.9 & 3.3 & 3.2 \\
\hline Office, Small & 2.9 & 3.3 & 3.2 \\
\hline Multifamily & 2.9 & 3.3 & 3.2 \\
\hline Single Family & 0.1 & 0.11 & 0.11 \\
\hline
\end{tabular}

\section{Methodology}

Of the 300 million sq ft added to the program each year, it is assumed that not all of the square footage per partner would be retrofit in one year, but that retrofits (and actual savings) occur evenly over four years. Penetration rates were

Community E nergy Program - 2 calculated using the square footage affected by the program as a percentage of the total square footage in the existing building stock.

The load reductions specified in the performance objective were applied to the baseline end-use loads to determine energy savings at the building level. These energy savings were translated into national energy savings using the penetration rates and building stodk within the target market and then adjusted using the most recent budget request information.

\section{Program/Technology Consumer Costs:}

- Cost of Conventional Technology: Average of $\$ 81 / \mathrm{sq} \mathrm{ft}$ for new commercial and multifamily; $\$ 0$ for existing buildings.

- Cost of BTS Technology: $\$ 82.60 / \mathrm{sq} \mathrm{ft}$ for new commercial and multifamily; \$3/ sq ft (2001 to 2009), increasing to $\$ 4 / \mathrm{sq} \mathrm{ft}$ (2010 to 2030) for existing buildings.

- Incremental Cost: 2\% above base for new buildings; \$3/sq ft (2001 to 2009) increasing to $\$ 4 /$ sq ft (2010 to 2030) for existing buildings.

\section{Non-Energy Benefits:(1)}

Revitalized neighborhoods and business districts, improved school facilities, better low-income housing, and positive economic impact from keeping dollars locally and increasing property values.

\section{Program Strategy (\% of budget): ${ }^{(1)}$}

- Research and Development - 0\%

- Market Transformation - 100\%

- Codes and Standards - 0\%.

\section{Sources:}

(1) FY 2002 Budget Data - Bucket Report for Rebuild America Program (includes Energy Smart Schools and Competitively Selected Community Program) (internal BTS document).

(2) FY 2003 data collection interview with Daniel Sze, August 20, 2001. 


\section{Information Outreach}




\section{Information Outreach}

Community Energy Program Decision Unit

\section{Program Objective:(1)}

The Information Outreach program provides BTS with the technical assistance needed to conduct the various planned activities that will educate target audiences. Specifically, the program conceptualizes, plans, and implements a systematic approach to the marketing and communication objectives and evaluation of the programs it supports.

\section{Long-Term Goal:(1)}

By 2010, the program's goal is to support long-term success in developing energy-efficient systems and processes and to improve technology transfer/information exchange.

\section{Market Segment: Methodology $\mathbf{y}^{(2)}$}

A slightly modified version of an evaluation conducted for this program was used to estimate this program's GPRA benefits. This section draws extensively from the report ${ }^{(3)}$ of that evaluation, which follows protocols used by major public relations firms and prominent measurement organizations. The major aspects of the evaluation protocols are as follows:

- Preparation (BTS activities)

- Adequacy of background information base for designing the program

- Appropriateness of the message and activity

- Quality of the message and activity presentations.

- Implementation (distribution effectiveness)

- Number of messages sent to media and activities designed

- Number of activities placed and implemented

- Number who receive messages and activities

- Number who are responsible for messages and activities.

- Impact (action taken)

- Number who learn message content

- Number who change opinions

- Number who change attitudes

- Number who behave as desired

- Number who repeat behavior

- Social and cultural change.

The methodology is summarized as follows:

1. Choose the measurable target audiences from the BTS strategic plan (e.g., homeowners, commercial builders,

\section{Information Outreach}

Program Type:

Information/Education

Target Market:

New residential and existing commercial in all climate zones

End Uses:

All end uses, all fuel types

Unit of Measurement:

Energy savings per budget dollar

Modeling Tool:

Spreadsheet

Program Manager:

Lani McRae

FY 2003 Benefits

Primary Energy Savings (TBtu)

\begin{tabular}{cccc}
2003 & 2005 & 2010 & 2020 \\
\hline 26.0 & 7608 & 69.1 & 63.6
\end{tabular}

Carbon Equivalent Reductions (MMTCE)

\begin{tabular}{cccc}
2003 & 2005 & 2010 & 2020 \\
\hline .438 & 1.344 & 1.276 & 1.123
\end{tabular}

Consumer Cost Savings (million \$)

\begin{tabular}{llll}
2003 & 2005 & 2010 & 2020 \\
\hline 161 & 464 & 437 & 451
\end{tabular}

Community Energy Program - 3 


\section{Information Outreach}

Community Energy Program Decision Unit

and building retrofit decisionmakers) who can implement a BTS strategy, tip, or technology.

2. Determine the energy-savings' potential of each representative in the target group from the BTS Core Data Book, (3) BTS programmatic experience, and EIA.

3. Count the total number of impressions from each distribution method and determine how many resulted from commercial builders, building retrofit decisionmakers, and individual homeowners (target group) (see the table).

4. Use industry-accepted norms to determine what percentage of the target audience who received the message are likely to change their opinion or behavior.

5. Multiply the results in step 4 for each distribution mechanism by the Btu savings' potential calculated for each target audience member in step 2.

The evaluation prepared for the program estimated a total primary energy savings over a $2^{1} / 2$-year period of $34.8 \mathrm{TBtu}$, resulting in annual energy savings of 13.92 TBtu. The evaluation assumed that savings lasted for 3 years, after which they are supplanted by activities the decisionmaker would have undertaken in any event. As a result, savings increase over three years to 41.76 TBtu $(13.92 \times 3)$ and then stay constant. In the evaluation, ${ }^{(2)}$ the savings were assumed to be $5 \%$ residential and $95 \%$ commercial, based on the types of decisionmakers the program reaches. The fuel distribution of the savings was assumed to match that of the rest of the BTS portfolio.

\section{Non-Energy Benefits:(1)}

The challenges of organizing information and communicating effectively are increased by new ideas and technologies, the diversity of stakeholders, and changing stakeholder needs, assumptions, and perceptions. The American public understands and takes into account the energy benefits of energy usage during purchases, giving the consumer more discretionary dollars.

\section{Program Strategy (\% of budget):(1)}

- Research and Development - 0\%

- Market Transformation - $100 \%$

- Codes and Standards - 0\%.

\section{Sources:}

(1) FY 2002 Budget Request - Data Bucket Report for I nformation Outreach Program (internal BTS document).

(2) Messersmith, J ., and S.A. Azim. August 2000. Communication Effectiveness Analysis for GPRA. Technologists, Inc.

(3) BTS Core Data Book. 1999 (internal document).

\begin{tabular}{|l|r|r|r|r|}
\hline \multicolumn{6}{|c|}{ Number of Instances a BTS Key Message was Seen or Heard } \\
\hline \multicolumn{1}{|c|}{ Deployment } & \multicolumn{1}{|c|}{ FY 2000 } & \multicolumn{1}{c|}{ FY 1999 } & \multicolumn{1}{c|}{ FY 1998 } & Cumulative Impact \\
\hline Conferences (attendees) & 46,300 & 66,650 & 1,000 & 113,950 \\
\hline Internet (page views) & 1 million & 2.4 million & 756,426 & 4.3 million \\
\hline Media (circulation & 763 million & 942 million & 1.6 trillion & 3.3 trillion \\
\hline Direct Mail (recipients) & 35,812 & 120,064 & 30,300 & 186,176 \\
\hline EREC* (recipients) & 17,783 & 58,984 & 68,294 & 145,061 \\
\hline Training (handouts) & 0 & 3,500 & 872 & 4,372 \\
\hline Hotline (calls) & 0 & 800 & 1,623 & 2,423 \\
\hline Totals & 764 million & 944 million & 1.6 trillion & 3.3 trillion \\
\hline${ }^{*}$ Energy Efficiency and Renewable Energy Clearinghouse. \\
\hline
\end{tabular}




\section{Training and Assistance for Codes}




\section{Training and Assistance for Codes}

\section{Program Objective:}

While the Training and Assistance for Codes program receives separate funding allocation, the program is characterized as part of the Residential Building Codes and Commercial Building Codes programs. The benefits for this program are broken out separately from those programs.

For information on this program, see the documentation for the Commercial Building Codes and Residential Building Codes programs.

\section{Training and Assistance for Codes}

Program Type:

Information/Education

Target Market:

New residential and commercial buildings in all climate zones

\section{End Uses:}

All end uses, all fuel types

Unit of Measurement:

Savings as the percentage of compliance improvement

Modeling Tool:

Spreadsheet

Program Manager:

Jean Boulin

Website:

http://www.energycodes.gov

FY 2003 Benefits

Primary Energy Savings (TBtu)

\begin{tabular}{llll}
2003 & 2005 & 2010 & 2020 \\
\hline 5.3 & 33.4 & 105.9 & 286.4
\end{tabular}

Carbon Equivalent Reductions (MMTCE)

\begin{tabular}{llll}
2003 & 2005 & 2010 & 2020 \\
\hline .102 & .614 & 1.803 & 5.152
\end{tabular}

Consumer Cost Savings (million \$)

\begin{tabular}{llll}
2003 & 2005 & 2010 & 2020 \\
\hline 33 & 217 & 813 & 2296
\end{tabular}




\section{Energy Star}




\section{Energy Star}

Energy Star Decision Unit

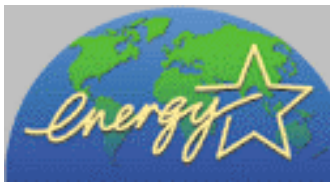

\section{Program Objective:(1)}

The Energy Star program increases the market penetration of high-efficiency appliances, windows, and lighting products through consumer education and voluntary industry partnerships. This program works closely with the private sector to bring new technology into the market through approaches such as high-volume purchases, utility program coordination, product testing, labeling, sales training, and provision of technical information to key market segments.

\section{Long-Term Goal:(1)}

The program's long-term goal is to achieve a sustained market share of high-efficiency appliances of $20 \%$ by 2010.

\section{Market Segment:}

\section{Performance Objective:}

- Displaced Technology: Conventional equipment, appliances, and lights.

- Performance Target: Varies by equipment type and size. The following represents a sample of typical Energy Star products on the market:

- Clothes Washers - Modified energy factor of 1.26 or greater

- Refrigerators - Must exceed the J uly 1, 2001, minimum federal standards by at least $10 \%$, modeled in NEMS as a refrigerator consuming $430 \mathrm{kWh} / \mathrm{yr}$

- Electric Water Heaters - Energy factor ranges from .95 to .96, modeled in NEMS as .96

- Gas Water Heaters - Energy factor of .60 to .65, modeled in NEMS as .64

- Room Air Conditioners - Must exceed the new federal standards (October 1,2000 ) by at least $10 \%$; the actual energy efficiency ratio depends on the size of the unit

- Compact Fluorescent Lights - Typical Product having efficacy of 40 to 60 lumens/watt

- Dishwashers - Energy factor of .65 or greater; typical product uses 400 to $450 \mathrm{kWh} / \mathrm{yr}$.

\section{Energy Star}

Program Type:

Market Transformation

Target Market:

Commercial sector and residential housing with $>\$ 25,000 / \mathrm{yr}$ incomes in all climate zones

\section{End Uses:}

Heating, cooling, water heating, lighting, and appliances

Unit of Measurement:

Load/efficiency per affected unit

\section{Modeling Tool:}

Appliances, Air Conditioning, and

Water Heating: NEMS

Compact Fluorescent Lights: spreadsheet.

\section{Program Manager:}

Bill Noel

Website:

http://www.energystar.gov

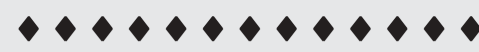

FY 2003 Benefits

Primary Energy Savings (TBtu)

\begin{tabular}{llll}
2003 & 2005 & 2010 & 2020 \\
\hline 15.2 & 41.1 & 169.0 & 568.1
\end{tabular}

Carbon Equivalent Reductions (MMTCE)

\begin{tabular}{llll}
2003 & 2005 & 2010 & 2020 \\
\hline .260 & .747 & 3.381 & 10.932
\end{tabular}

Consumer Cost Savings (million \$)

\begin{tabular}{llll}
2003 & 2005 & 2010 & 2020 \\
\hline 107 & 288 & 1375 & 5373
\end{tabular}




\section{Energy Star}

Energy Star Decision Unit

\section{Target Market}

- Market Description: Determined by program equipment. For FY 2003, the following equipment is characterized (residential):

- Clothes washers

- Refrigerators

- Electric water heaters

- Gas water heaters

- Room air conditioners

- Compact fluorescent lights

- Dishwashers.

\section{Methodology}

Clothes washers, refrigerators, electric water heaters, gas water heaters, dishwashers, and room air conditioners were modeled in NEMS using input from EIA's Annual Energy Outlook 2001. (2) NEMS inputs were determined such that a $20 \%$ market share is obtained for Energy Star level appliances.

Energy Star compact fluorescent lights were modeled in BESET and assumed that they would capture $10.5 \%$ of incandescent sales in the residential sectors by 2020 (based on market penetration goal of capturing $20 \%$ of the installed base). Energy Star compact fluorescent lights were assumed to penetrate the high-use part of the market where $76.4 \%$ of the residential lighting energy is consumed (e.g., rooms such as kitchens and bathrooms). The sockets in high-use areas $(28.3 \%$ of the total sockets) will use roughly the same fraction of the lamps (i.e., $28.3 \%$ of the sockets consume $76.4 \%$ of the lighting energy use). A sales fraction of $10.5 \%$ will yield a long-term installed base of $20 \%$.

This activity was assumed not to occur without DOE funding; therefore, the NAS (acceleration-to-market) methodology was not applied.

\section{Non-Energy Benefits:(1)}

Increased comfort for residential homeowners and office workers and higher profits for manufacturers.

\section{Program Strategy (\% of budget): ${ }^{(1)}$}

- Research and Development - 1\%

- Market Transformation - 99\%

- Codes and Standards - 0\%.

\section{Sources:}

(1) FY 2002 Budget Request - Data Bucket Report for Energy Star Program (internal BTS document).

(2) Annual Energy Outlook 2001. 2000. Energy Information Administration, Washington, D.C. 


\section{Residential Buildings}




\section{Residential Technology R\&D}




\section{Program Objective:(1)}

The Residential Technology R\&D program consolidates the formerly separate systems engineering programs of Building America, Industrialized Housing, Passive SoIar Buildings, and Indoor Air Quality programs and existing building research into a comprehensive program to accelerate the introduction of highly efficient building technologies and practices through $R \& D$ of advanced systems for production builders.

\section{Long-Term Goal:(1)}

The program's long-term goal is to develop advanced systems to improve the energy performance of over 300,000 of the 1 million homes that will be built in 2010. The increased performance will allow the homes to use $50 \%$ less energy for space conditioning and water heating than typical homes built in 1993.

\section{Market Segment:}

\section{Performance Objective:(2)}

- Displaced Technology: Current design/building practices.

- Performance target: $50 \%$ load reduction in space heating and cooling and water heating by 2010 .

\section{Target Market ${ }^{(1)}$}

- Market Description: New singlefamily, multifamily, and manufactured housing units with homeowners having $>\$ 25,000$ annual income in all climate zones. The market includes primarily new single family homes, multifamily infill, HUD code homes, and small commercial buildings. Existing homes are to benefit from new technologies and improved construction practices developed for new homes.

- Size of Market: Each year 1.2 million new housing units are built and use 3.19 quad. These units are primarily owner occupied.

- Market Introduction: 1997.(2) This activity was assumed not to occur without DOE funding; therefore, the NAS (acceleration-to-market) methodology was not applied.

- Market Penetration Goal:(3) See the table below.

\section{Residential Technology} R\&D

Program Type:

Whole Building

Target Market:

New single-family, multifamily, and manufactured housing units with homeowners having $>\$ 25,000 / y r$ income in all climate zones

\section{End Uses:}

All end uses, all fuel types

Unit of Measurement:

$\%$ change in load per household

Modeling Tool:

BESET

Program Manager:

George James/Jon Stone

Website:

http://www.eren.doc.gov/buildings/ builidng_america

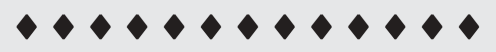

\section{FY 2003 Benefits}

Primary Energy Savings (TBtu)

\begin{tabular}{llll}
2003 & 2005 & 2010 & 2020 \\
\hline 0.2 & 0.9 & 8.7 & 21.3
\end{tabular}

Carbon Equivalent Reductions (MMTCE)

\begin{tabular}{llll}
2003 & 2005 & 2010 & 2020 \\
\hline .003 & .015 & .150 & .358
\end{tabular}

Consumer Cost Savings (million \$)

\begin{tabular}{llll}
2003 & 2005 & 2010 & 2020 \\
\hline 1 & 6 & 64 & 1820
\end{tabular}

Residential Buildings I ntegration - 1 


\section{Residential Technology R\&D}

Residential Buildings Integration Decision Unit

\begin{tabular}{|c|c|c|}
\hline \multicolumn{3}{|c|}{$\begin{array}{c}\text { Market Penetration Curve: } \\
\text { FY 2003 Estimate }\end{array}$} \\
\hline Year & \# Builders & $\begin{array}{c}\text { Annual \# } \\
\text { Homes }\end{array}$ \\
\hline 2003 & 60 & 7,200 \\
\hline 2004 & 76 & 19,800 \\
\hline 2005 & 96 & 18,900 \\
\hline 2006 & 121 & 33,075 \\
\hline 2007 & 152 & 34,729 \\
\hline 2008 & 192 & 44,762 \\
\hline 2009 & 242 & 47,000 \\
\hline 2010 & 305 & 50,916 \\
\hline 2011 & 384 & 50,916 \\
\hline 2012 & 484 & 45,825 \\
\hline 2013 & 610 & 35,132 \\
\hline 2014 & 769 & 30,916 \\
\hline 2015 & 969 & 29,216 \\
\hline 2016 & 1,220 & 26,795 \\
\hline 2017 & 1,538 & 23,669 \\
\hline 2018 & 1,938 & 19,882 \\
\hline 2019 & 2,441 & 15,508 \\
\hline 2020 & 3,076 & 10,649 \\
\hline & & \\
\hline
\end{tabular}

\section{Methodology}

For any one year, energy savings for this program are calculated by multiplying the number of homes built with Building America techniques that year times the percent savings per home. Added to these savings are the energy savings, in that year, for Building America homes built in previous years (within the analysis period, any savings resulting from homes built before 2003 are not included).
Program/Technology Consumer Costs:

- Cost of Conventional Technology

(average price per household):

- Single Family: $\$ 126,700$

- Multifamily: $\$ 74,900$

- Manufactured Home: \$41,100.

- Cost of BTS Technology: $2 \%$ above conventional cost

- Incremental Cost (average price per household):

- Single Family: $\$ 2,534$

- Multifamily: $\$ 1,498$

- Manufactured Home: $\$ 822$.

\section{Non-Energy Benefits:(1)}

Consumer savings of $\$ 148$ million by 2010; improved comfort, durability, and occupant health from better indoor air quality, reduced onsite generated waste, better sustainability, and reduced maintenance.

Program Strategy (\% of budget): $:^{(1)}$

- Research and Development - 90\%

- Market Transformation - $10 \%$

- Codes and Standards - 0\%.

\section{Sources:}

(1) FY 2002 Budget - Data Bucket Template Residential Building Integration $R \& D$ (internal BTS document).

(2) FY 2002 GPRA Program Characterization (internal document).

(3) Based on I mpacts spreadsheet developed by Ren Anderson, August 10, 2000. 


\section{Residential Building Codes}




\section{Program Objective:(1)}

The Residential Building Codes program improves the energy efficiency of new residential buildings and additions and alterations to existing buildings. The objective will be accomplished by improving the energy-efficiency provisions of building codes and applicable standards that affect residential construction and by providing technical assistance for implementing those codes and standards.

\section{Long-Term Goal:(1)}

The program's long-term goal for applicable residential building codes is to incorporate the most technologically feasible, economically justified energy conservation measures. Another goal is to provide state and local governments with the needed technical tools and information for adopting, using, and enforcing efficient building codes for residential construction.

These goals can be quantified in terms of the percentage improvements in efficiency in existing residential building codes, the number of jurisdictions that adopt and successfully implement these codes, the number of new houses built in compliance with the new code, and the number of renovations and additions to existing buildings complying with the code.

\section{Market Segment:}

\section{Target Market ${ }^{(1)}$}

- Market Description: New residential buildings that are generally three stories or less in height (taller buildings fall under the commercial buildings program) and additions and alterations to existing buildings. The program can affect residences' major energy end uses: heating, cooling, water heating, and possibly lighting energy in the near future. All areas of the country are affected because the model building codes and standards cover all climate zones. Household income is not a discrimination of the target market because building codes cover housing at all costs and income levels. Energyefficiency improvements via codes have repeatedly demonstrated a net positive cash flow to the new home buyer within five years, thus actually improving household income.

\section{Residential Building Codes}

Program Type:

Information/Education

Target Market:

New residential buildings in all

climate zones

End Uses:

All end uses, all fuel types

Unit of Measurement:

$\%$ load reduction

Modeling Tool:

Spreadsheet

Program Manager:

Steve Walder

Website:

http://www.eren.doe.gov/buildings/ codes_standards/bldgstds.htm

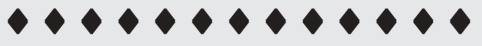

FY 2003 Benefits

Primary Energy Savings (TBtu)

\begin{tabular}{llll}
2003 & 2005 & 2010 & 2020 \\
\hline 0.1 & 1.2 & 8.7 & 32.5
\end{tabular}

Carbon Equivalent Reductions (MMTCE)

\begin{tabular}{cccc}
2003 & 2005 & 2010 & 2020 \\
\hline .002 & .020 & .136 & .521
\end{tabular}

Consumer Cost Savings (million \$)

\begin{tabular}{llll}
2003 & 2005 & 2010 & 2020 \\
\hline 1 & 9 & 65 & 247
\end{tabular}

Residential Buildings I ntegration - 3 
- Size of Market: About 1.4 million building permits were issued last year, over a million for singlefamily dwellings. Although not all jurisdictions currently have energy-efficiency building codes in place, about half of all new residential construction is conservatively estimated to come under building code requirements. Also, consumers spend several billion dollars a year remodeling and renovating private residences, about half of which could presently be covered by an energy code. One market not currently covered by codes is manufactured homes, which fall under HUD's jurisdiction and regulations.

\section{Methodology}

The FY 2003 GPRA estimate was based on increased compliance with existing codes, accelerated adoption of the IECC 2001 code (to comply with EPAct), and the future development of more stringent building codes. The energy savings' methodology was performed at a state level to better link changes in the national standards (e.g., IECC 2001) with variations in climate by states and differences among states in their adoption and enforcement of building codes. The discussion below uses national averages of some of the key assumptions related to adoption and compliance to help summarize the methodology.

The principal difference between the 1995 Model Energy Code and the IECC 2001 pertains to the solar heat gain requirements for windows. Based on a series of simulations for various locations around the United States, the percentage reduction in cooling load was estimated to be about 15\%. This requirement increases the heating load by a small amount, about 2\% nationally. (The requirement itself is restricted to the southern tier of states). The GPRA estimates were partly based on states' accelerated schedule of adoption of the IECC 2001 code. Through the efforts of the Building Standards \& Guidelines Program, 31 states were assumed to have adopted the standard by the end of 2005. The program was assumed to accelerate the adoption of the standard by an average of four years nationwide.

The IECC's ongoing activities were assumed to lead to more stringent residential standards in the future. DOE was assumed to play a major role in developing the analytical and economic basis for such standards. For the GPRA process, these activities were subsumed in a single upgrade of the IECC standard assumed to become available in the latter part of the current decade. Based on discussions with BTS staff, PNNL assumed these upgrades reduced heating and cooling loads in new residential structures by $10 \%$. Without these activities, the analysis assumed that the same standard would be adopted, on average, six years later.

The program's activities also were assumed to improve compliance rates for existing and future building codes. Compliance is increased through better familiarity with the codes over time, simplifications to the code while maintaining stringency, and the availability and increased use of compliance tools by builders and enforcement officials. Compliance rates, with and without the program, were estimated for the existing, IECC 2001, and the succeeding code as discussed above. As a national average, compliance with existing codes was estimated at $43 \%$ in 2000 , rising to $52 \%$ without the program and $70 \%$ by 2010 with the program.

The compliance with the several key provisions in the IECC 2001 (compared with MEC 95) was expected to be higher from the outset. On average, the compliance was estimated to be $63 \%$ in the year of the 
Residential Building Codes

Residential Buildings Integration Decision Unit

adoption. Ten years later, compliance rates were assumed to increase to $70 \%$ without the program and $78 \%$ with the program. For homes that do not comply with the standard, only half of the incremental energy savings were assumed to be achieved by adopting the IECC 2001.

The analysis assumed that the greater stringency of a new standard to become available in the 2010 time frame will result in somewhat lower compliance when states first adopt it. I nitial compliance was assumed to be about $48 \%$ at the time of adoption, rising to $58 \%$ without the program and $60 \%$ with the program after the first ten years. As for the IECC 2001, the energy savings in units that do not comply were assumed to be $50 \%$ of that in units that comply fully with the code.

\section{Non-Energy Benefits: ${ }^{(1)}$}

Lower utility bills, improved indoor comfort, lower home maintenance and repair activities, and reduced pollution from burning fossil fuels and generating electricity, which improves air quality and mitigates the negative impacts of global warming.

\section{Program Strategy (\% of bud- get):(1)}

- Research and Development - 0\%

- Market Transformation - 0\%

- Codes and Standards - 100\%.

\section{Sources:}

(1) FY 2002 Budget - Data Bucket Report for Residential Building Integration $R \& D$ Program (internal BTS document). 


\section{Commercial Buildings}




\section{Commercial Technology R\&D}




\section{Program Objective:}

The Commercial Technology $R \& D$ program develops and demonstrates advanced technologies, controls, and equipment in collaboration with the design and construction community. The program focuses on advancing integrated technologies and practices to optimize whole-building energy performance. The program reduces energy use in commercial and multifamily buildings by promoting practices that help ensure the industry constructs buildings as designed and operates them at or near the optimum level of performance.

\section{Long-Term Goal:}

The program's long-term goal is to improve by $30 \%$ the energy efficiency of the nation's new commercial buildings and existing buildings by 20\% compared with 1996.

\section{Market Segment:}

\section{Performance Objective:}

- Displaced Technology: Conventional design/ building practices.

- Performance Target: Reduce heating and cooling loads by $30 \%$ in new construction and by $20 \%$ in existing units compared with 1996.

- By 2010 reduce heating and cooling loads by $50 \%$ in new construction and by $30 \%$ in existing units.

- By 2020 reduce heating and cooling loads by $60 \%$ in new construction and $40 \%$ in existing units.

\section{Target Market ${ }^{(1)}$}

- Market Description: Commercial buildings that the program's research products are most likely to impact; includes all commercial buildings except those using very low amounts of energy $(<10 \%$ of average building consumption) and those having very low energy-use intensities ( $<50 \%$ of the average energy-use intensity).

- Market Introduction: 1996 (inception date of program is 1977). This activity was assumed not to occur without DOE funding; therefore, the NAS (acceleration-to-market) methodology was not applied.

- Market Penetration Goal: To penetrate $60 \%$ of new commercial and multifamily construction by 2020 in combination with the Design Strategies and

\section{Commercial Technology $R \& D$}

Program Type:

Whole Building

Target Market:

New and existing commercial and residential multifamily units in all climate zones

\section{End Uses:}

Heating and cooling

Unit of Measurement:

$\%$ change in load

Modeling Tool:

BESET

\section{Program Manager: \\ Dru Crawley}

\section{Website:}

http://www.eren.doe.gov/buildings/ highperformance/

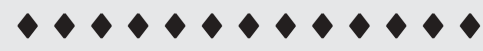

FY 2003 Benefits

Primary Energy Savings (TBtu)

\begin{tabular}{llll}
2003 & 2005 & 2010 & 2020 \\
\hline 1.0 & 4.0 & 32.5 & 138.7
\end{tabular}

Carbon Equivalent Reductions (MMTCE)

\begin{tabular}{cccc}
2003 & 2005 & 2010 & 2020 \\
\hline .017 & .070 & .599 & 2.466
\end{tabular}

Consumer Cost Savings (million \$)

\begin{tabular}{llll}
2003 & 2005 & 2010 & 2020 \\
\hline 6 & 25 & 215 & 1035
\end{tabular}

Commercial Buildings I ntegration - 1 


\section{Commercial Technology R\&D}

Commercial Buildings Integration Decision Unit

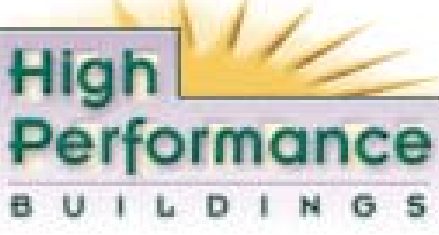

Assistance program. In addition, penetrate $20 \%$ of existing commercial and multifamily buildings by 2020 .

\section{Non-Energy Benefits:}

Reduced operation and maintenance expenses, improved indoor environmental quality, increased property asset value, higher tenant satisfaction and retention rates, and increased product sales.

Program Strategy (\% of budget): ${ }^{(2)}$

- Research and Development - 90\%; the bulk of funding is research-focused on high-performance building techniques, fault detection and diagnostics, indoor environmental quality, building commissioning, and integrated building systems.
- Market Transformation - 10\%; small initiatives were established with the Building America and Rebuild America programs to incorporate high-performance building research into their deployment activities to create direct linkages between the separate $R \& D$ and deployment programs, which are focused on the same target markets.

- Codes and Standards - 0\%.

\section{Sources:}

(1) Interview with Program Manager, Dru Crawley, August 2001.

(2) FY 2002 Budget - Bucket Report for Commercial Buildings Integration $R \& D$ Program (internal BTS document). 


\section{Commercial Building Codes}




\section{Program Objective:(1)}

The Commercial Buildings Codes program promotes energy efficiency by promulgating federal energy codes while working with model code groups to upgrade building codes and standards that state and local jurisdictions can adopt and that are applicable to all new commercial and high-rise residential buildings, permitted renovations, and additions. This program targets all new commercial buildings, with federal construction representing about $3 \%$ of all new construction.

\section{Long-Term Goal:(1)}

The program's long-term goal is to reduce total energy use by $30 \%$ to $35 \%$ in all new commercial building construction by 2010. Energy use will be reduced by the widespread adoption of building energy codes by all states and U.S. jurisdictions. The program is also establishing an educational library consisting of manuals, software, and training support for both voluntary commercial and federal commercial energy-efficiency codes.

\section{Market Segment:}

\section{Target Market}

- Market Description: All new commercial and multifamily high-rise buildings and all permitted additions and renovations to those buildings.

- Size of Market: About 4.7 million buildings representing 62.9 billion sq ft of floor space, consuming about 7.7 quad of energy onsite and costing $\$ 85.2$ billion annually. The federal sector represents about $2.3 \%$ overall of new commercial building construction.

Energy savings from this program and the related Technical Assistance for Codes result from basic improvements in overall energy efficiency of commercial buildings. The present funding method channels funding for conducting research activities for new codes and for developing compliance tools - primarily for existing codes - through BTS's Building Research and Standards area. Funding for Training and Technical Assistance for state building energy codes (related primarily to existing codes) is channeled through BTS Building Technology Assistance area. Benefits cannot be clearly allocated to either area.

\section{Commercial Buildings Codes}

Program Type:

Information/Education

Target Market:

New commercial buildings in all climate zones

\section{End Uses:}

All end uses, all fuel types

Unit of Measurement:

$\%$ change in load

Modeling Tool:

Spreadsheet

Program Manager:

Ron Majette

Website:

http://www.eren.doe.gov/buildings/ codes_standards/bldgstds.htm

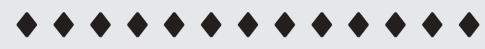

FY 2003 Benefits

Primary Energy Savings (TBtu)

\begin{tabular}{llll}
2003 & 2005 & 2010 & 2020 \\
\hline 0.0 & 0.0 & 9.0 & 100.1
\end{tabular}

Carbon Equivalent Reductions (MMTCE)

\begin{tabular}{llll}
2003 & 2005 & 2010 & 2020 \\
\hline .000 & .000 & .184 & 1.948
\end{tabular}

Consumer Cost Savings (million \$)

\begin{tabular}{llll}
2003 & 2005 & 2010 & 2020 \\
\hline 0 & 0 & 60 & 785
\end{tabular}

Commercial Buildings Integration - 3 


\section{Commercial Buildings Codes}

Commercial Buildings Integration Decision Unit

Barring future guidance from DOE, benefits for FY 2002 were assumed to be allocated according to the ratio of actual funding levels.

The description of the methodology below also pertains to Technical Assistance for Codes.

The program's impact is primarily through two avenues: 1) developing and supporting standard/code changes to improve energy efficiency in commercial building structures and 2) developing tools that can ease the adoption of new codes, and through their use, support improvements in compliance and enforcement of code provisions. Tools take the form of code compliance software, computer-based training tools for building energy codes, and tools for implementing non-computer-based codes.

Changes to building standards and codes are primarily supported by research efforts to review existing codes and specific targeted areas of building energy use and the adoption of code modifications that promote cost-effective reductions in these energy use areas. The research work has typically taken place on three fronts: support for ASHRAE/IES 90.1, "Energy Standard for New Commercial Buildings";(2) support for developing federal building codes, and support for developing the IECC.

The FY 2003 GPRA estimate was based on increased compliance with existing codes, accelerated adoption of the ASHRAE 90.1-1999 standard $^{(3)}$ (to comply with EPAct), and the future development of more stringent building codes. The energy savings' methodology was performed at a state level to better link changes in the standards (e.g., IECC 2001) with variations in climate by states and differences among states in their adoption and enforcement of building codes. The discussion below uses national averages of some of the key assumptions related to adoption and compliance to help summarize the methodology.

Commercial Buildings I ntegration - 4
The principal differences between the ASHRAE 90.1-1989(4) and 90.1-1999 standards relate to requirements for better windows, reduced installed wattage for lighting, and more efficient heating and cooling equipment. The savings from improved equipment were not included in this program's savings estimates because they are reflected in the separate EPAct Standards Program.

Based on a series of simulations that include various U.S. locations and that were developed spedifically to evaluate the two ASHRAE standards (often referred to as the "determination" study ${ }^{[5]}$ ), the average reduction in site energy use was estimated to be about $3.5 \%$ or about 2 MMBtu/square foot. The GPRA estimates were partly based on states' accelerated adoption schedule of the ASHRAE 90.1-1999 standard. Through the efforts of the Building Standards \& Guidelines Program, 35 states were assumed to have adopted the standard by the end of 2005. The program was assumed to accelerate the adoption of the standard by an average of four years nationwide.

The ongoing activities of the ASHRAE 90.1 committee were assumed to lead to more stringent commercial building standards in the future. DOE was assumed to play a major role in developing the analytical and economic basis for such standards. For the GPRA process, these activities were subsumed in a single upgrade of the ASHRAE standard assumed to be come available in the latter part of the current decade. The GPRA analysis assumed that the overall result of these upgrades is to reduce electricity consumption by $10 \%$ and natural gas consumption by $2 \%$ in new commercial buildings. The adoption of this standard by many states by 2010 also depends on the program's continuing activities to assist states in the adoption (and compliance) process. Without these activities, the analysis assumed that the same standard would be adopted, on average, six years later. 


\section{Commercial Buildings Codes}

Commercial Buildings Integration Decision Unit

The program activities were also assumed to improve compliance rates for existing and future building codes. Compliance is increased through increased familiarity with the codes over time, simplifications to the code while maintaining stringency, and the availability and increased use of compliance tools by builders and enforcement officials. Compliance rates, with and without the program, were estimated for the existing code, a code based on ASHRAE 90.1-1999, and a future standard as discussed above. On a national average, compliance with existing codes was estimated at $45 \%$ in 2000 , rising to $54 \%$ without the program, and $70 \%$ by 2010 with the program.

The compliance with the several key provisions in ASHRAE 90.1-1999 (compared with 90.1-1989) was expected to be higher from the outset. On average, the compliance was estimated to be $50 \%$ in the year of the adoption. Ten years later, compliance rates were assumed to increase to $60 \%$ without the program and $70 \%$ with the program. For buildings that do not comply with the standard, only half of the incremental energy savings were assumed to be achieved by adopting the ASHRAE 90.1-1999.

The analysis assumed that the simplifications in the ASHRAE 90.1-1999 standard will be extended to the new standard and will result in somewhat higher compliance when first adopted by states. Initial compliance was assumed to be about $55 \%$ at the time of adoption, rising to $64 \%$ without the program and $73 \%$ with the program after the first ten years. As for the 90.1-1999 standard, the energy savings in buildings that do not comply were assumed to be $50 \%$ of that in buildings that comply fully with the code.

\section{Non-Energy Benefits:}

Improved environment and more comfortable buildings.

\section{Program Strategy}

(\% of budget): (2)

- Research and Development - 0\%

- Market Transformation - 0\%

- Codes and Standards - 100\%.

\section{Sources:}

(1) FY 2002 Budget - Data Bucket Report for Commercial Building Codes Program (internal BTS document).

(2) ASHRAE/IES 90.1, "Energy Standard for New Commercial

Buildings,"American Society of Heating, Refrigeration, and Air-Conditioning Engineers and Illuminating Engineering Society.

(3) ASHRAE 90.1-1999, “Energy Standard for Buildings Except Low-Rise Residential Buildings," American Society of Heating, Refrigeration, and Air-Conditioning Engineers.

(4) ASHRAE Standard 90.1-1989, “Energy Efficient Design of New Buildings Except New Low-Rise Residential Buildings," American Society of Heating, Refrigeration, and AirConditioning Engineers.

(5) U.S. Department of Energy. 2002. "Commercial Buildings Determinations, Explanation of the Analysis and Spreadsheet (90_1savingsanalysis.xls)." 〈http:/ /www. energycodes.gov/implement/ determinations_com.stm> 


\section{Building Equipment, \& Materials}




\section{Lighting R\&D}




\section{Lighting R\&D}

Building Equipment and Materials Decision Unit

\section{Program Objective:(1)}

The Lighting R\&D program develops and accelerates the introduction of advanced lighting technologies. A new program initiative, solid-state lighting, will foster the introduction of solid-state lighting and will seek to achieve the following for lighting:

- Significantly greater efficiency than conventional sources

- Easy integration into building systems of the future

- Able to provide the appropriate color and intensity for any application

- Lasting for 20,000 to 100,000 hours

- Able to readily supplement natural sunlight.

\section{Long-Term Goal:(1)}

The program's long-term goal is to reduce lighting energy use by $50 \%$ by 2020 .

\section{Market Segment}

\section{Target Market}

- Market Description: All commercial buildings, with some products being introduced into residential buildings.

- $\quad$ Size of Market: Lighting uses 26\% (3.9 quad) of the primary energy used in commercial buildings, which had a building stock of -63 billion sq ft in 2000 .

\section{Methodology}

The energy savings from the lighting program was generally based on the BTS Program Manager's judgment on the probable penetration of specific lighting technologies. However, the resulting savings are formally calculated from the NEMS commercial energy module. The capital costs of the technologies are adjusted to achieve approximate congruence with the external penetration assumptions.

For FY 2003, for the first time savings were estimated from BTS activities designed to increase the adoption and effectiveness of lighting controls. For this program component, a spreadsheet was used to develop the energy-savings' estimates. For all program technologies, the market introduction date was far enough out in the future that the NAS (accelerationto-market) methodology was not applied.

\section{Weatherization Program}

Program Type:

Equipment Efficiency

Target Market:

Potentially all sectors and all

climate zones (primarily impacts commercial sectors and higherincome residential)

End Uses:

Lighting and electricity

Unit of Measurement:

Lumen/watt

Modeling Tool:

NEMS

Program Manager:

Ron Lewis

FY 2003 Benefits

Primary Energy Savings (TBtu)

\begin{tabular}{llll}
2003 & 2005 & 2010 & 2020 \\
\hline 0.5 & 2.5 & 19.1 & 365.9
\end{tabular}

Carbon Equivalent Reductions (MMTCE)

\begin{tabular}{llll}
2003 & 2005 & 2010 & 2020 \\
\hline .000 & .009 & .391 & 7.12
\end{tabular}

Consumer Cost Savings (million \$)

\begin{tabular}{llll}
2003 & 2005 & 2010 & 2020 \\
\hline 3 & 16 & 127 & 2871
\end{tabular}

Building Equipment and Materials - 1 


\section{Lighting R\&D}

\section{Building Equipment and Materials Decision Unit}

\section{Solid-State Lighting and Two-Photon Phosphors}

For the solid-state lighting initiative, key assumptions concerning the likely dates of introduction and the expected efficacies were influenced by two sources: 1) a white paper, "The Case for a National Research Program on Semiconductor Lighting,"(2) prepared by Hewlett-Packard and Sandia National Laboratories staff for an industry forum in 1999; and 2) a more extended study done by A.D. Little for BTS in early $2001 ; ;^{(3)}$ the study used some of the basic assumptions in the white paper in developing some scenarios related to solid-state lighting.

As was done in FY 2002, the NEMS model was used to project savings from the lighting program. The current model allows the BTS technology to compete with a wide variety of technologies. The issue is complicated by EIA's use of functions to represent dedining costs of new technologies. Accordingly, the same general approach was followed as for the FY 2002 effort in which costs of the BTS technologies were adjusted to achieve some congruence with the expected penetration rates. If some reasonable basis for future costs can be developed, these costs can be put into the model to determine economic viability.

For FY 2003, the lighting estimates were based on NEMS projections that included two technologies supported by BTS: solid-state lighting and the two-photon phosphor lamp. In NEMS, each lighting technology is characterized by an efficacy level (lumen/watt), a capital cost (\$/1000 lumens), and an annual cost of lamps (\$/1000 lumens). For new technologies, the capital costs can be reduced along a logistic-shaped curve. The NEMS model divides the commercial lighting market into four major groups: incandescent (point source), 4-foot fluorescent, 8-foot fluorescent, and high-intensity point source (outdoor lighting). Solid-state lighting was assumed to penetrate the first three market groupings; the two-photon phosphor lamp was assumed to compete only with the fluorescent lighting groups.

Given the cost assumptions, the NEMS model chose among these technologies for each building type in each census division. For each group, the market was assumed to be further segmented, where each segment was characterized by a different discount rate in its decisionmaking criteria. Within each segment, a lighting technology was selected based on minimum annualized cost.

Table 1 summarizes the cost inputs for some key solid-state lighting technologies used in NEMS for FY 2003. The performance assumptions for solid-state lighting fall between the baseline and technology breakthrough cases in the Sandia and A.D. Little reports. . $^{(2,3)}$ The studies' baseline case predicts an efficacy of 45 lumen/watt in 2010 for a medium-color rendering index light-emitting diode source (as provided by most current fluorescent tubes). The efficacy increases to about 50 lumen/watt by 2015 . In the technology breakthrough case, the efficacy for a medium-color rendering index source was assumed to reach 110 and 120 lumen/watt by 2010 and 2015, respectively. As the table shows, the FY 2003 GPRA estimates were based on the efficacy of solid-state lighting reaching 60 and 85 lumen/watt in 2010 and 2015, respectively.

Table 2 summarizes the cost inputs for the two-photon phosphor program. An efficacy of 120 lumen/watt was assumed to be achieved by 2010, based partly on discussions with BTS Program Managers. The modeling followed a simpler approach compared with solid state only a single efficacy was assumed for the model's time horizon.

For FY 2003, Table 3 summarizes the results of the NEMS-generated market shares, which relate to the sales of new and replacement 


\section{Lighting R\&D}

\section{Building Equipment and Materials Decision Unit}

\begin{tabular}{|c|c|c|c|c|c|c|}
\hline \multirow{2}{*}{$\begin{array}{l}\text { Lighting } \\
\text { Technology }\end{array}$} & \multirow{2}{*}{$\begin{array}{c}\text { Efficacy } \\
\text { (lumen/ } \\
\text { watt) }\end{array}$} & \multicolumn{4}{|c|}{ Capital Cost ( $\$ / 1000$ lumen) } & \multirow{2}{*}{$\begin{array}{c}\text { Annual } \\
\text { Operating Cost } \\
\text { (\$/1000 lumens) }\end{array}$} \\
\hline & & 2005 & 2010 & 2015 & 2020 & \\
\hline \multicolumn{7}{|l|}{ Incandescent } \\
\hline Incandescent & 15.6 & 34.02 & 34.02 & 34.02 & 34.02 & 10.72 \\
\hline Compact Fluorescent Light & 66.7 & 38.62 & 37.74 & 37.17 & 36.79 & 6.21 \\
\hline Solid-State Lighting, 2008 & 40 & 68.00 & 67.83 & 66.13 & 63.22 & 0.51 \\
\hline Solid-State Lighting, 2010 & 60 & NA & 65.00 & 63.85 & 61.10 & 0.51 \\
\hline Solid-State Lighting, 2015 & 85 & NA & NA & 58.00 & 56.26 & 0.51 \\
\hline \multicolumn{7}{|l|}{ 4-Foot Fluorescent } \\
\hline F32T8 Electronic & 76.8 & 23.84 & 21.99 & 21.89 & 21.83 & 0.75 \\
\hline Solid-state Lighting, 2010 & 60 & NA & 26.00 & 25.54 & 24.44 & 0.51 \\
\hline Solid-state Lighting, 2015 & 85 & NA & NA & 22.00 & 21.34 & 0.51 \\
\hline \multicolumn{7}{|l|}{ 8-Foot Fluorescent } \\
\hline F96T12 Electronic & 81.8 & 7.49 & 14.81 & 14.74 & 14.70 & 0.54 \\
\hline Solid-state Lighting, 2010 & 60 & NA & 20.00 & 19.65 & 18.80 & 0.51 \\
\hline Solid-state Lighting, 2015 & 85 & NA & NA & 15.00 & 14.55 & 0.51 \\
\hline
\end{tabular}

\begin{tabular}{|c|c|c|c|c|c|c|}
\hline \multirow[b]{2}{*}{$\begin{array}{c}\text { Lighting Technology } \\
\text { (4-ft fluorescent) }\end{array}$} & \multirow{2}{*}{$\begin{array}{c}\text { Efficacy } \\
\text { (lumens/ } \\
\text { watt) }\end{array}$} & \multicolumn{4}{|c|}{ Capital Cost ( $\$ / 1000$ lumen) } & \multirow[b]{2}{*}{$\begin{array}{c}\text { Annual Operating } \\
\text { Cost (\$/1000 lumens) }\end{array}$} \\
\hline & & 2005 & 2010 & 2015 & 2020 & \\
\hline F32T8 Electronic & 76.8 & 22.18 & 21.99 & 21.88 & 21.83 & 0.75 \\
\hline $\begin{array}{l}\text { Two-photon phosphor } \\
\text { (2010 introduction) }\end{array}$ & 120 & NA & 27.00 & 24.30 & 22.20 & 0.51 \\
\hline
\end{tabular}

equipment in the year shown. For solid-state lighting, the penetrations are significant after 2015, with the new technology capturing nearly half the new sales of lighting equipment. These penetration rates fall between the 2001 A.D. Little study's's ${ }^{(3)}$ technology and price breakthrough scenarios (although closer to the latter). In the latter scenario, the penetration of solid-state lighting reaches $-60 \%$ in 2015 , compared with $<10 \%$ in the technology breakthrough case.

The market shares for the two-photon phosphor fluorescent lamps rise more steadily over the decade following their assumed 2010 market introduction. Because they are significantly more efficient than current fluorescent sources, they were assumed to capture a majority of the market by the end of the decade (in NEMS, the assumed price paths yielded $60 \%$ in the large 4-foot market and $37 \%$ in the 8 -foot market).

\section{Lighting Controls}

Various field studies have shown that aggressively implementing lighting controls, primarily using occupancy and daylighting controls, can save from $20 \%$ to $40 \%$ of lighting energy use. BTS is supporting the development of more advanced systems -through both re search and field testing - that will further reduce energy used for lighting in commercial buildings. BTS support of research to evaluate the interrelationship between human vision and efficient light use will also contribute to future energy savings. 


\section{Lighting R\&D}

Building Equipment and Materials Decision Unit

\begin{tabular}{|c|c|c|c|c|}
\hline \multicolumn{5}{|l|}{ Solid-State Lighting } \\
\hline Incandescent & 2005 & 2010 & 2015 & 2020 \\
\hline Incandescent 1170 lumen, 75 watt & 0.27 & 0.27 & 0.27 & 0.22 \\
\hline Compact fluorescent light (low-end 1200 lumen, 20 watt) & 0.17 & 0.17 & 0.18 & 0.22 \\
\hline Compact fluorescent light (1200 lumen, 20 watt) & 0.56 & 0.44 & 0.08 & 0.02 \\
\hline Solid-state Lightingñ2005 Intro (40 watt/lumen) & 0.0 & 0.0 & 0.0 & 0.0 \\
\hline Solid-state Lightingñ2010 Intro (60 watt/lumen) & NA & 0.12 & 0.0 & 0.0 \\
\hline Solid-state Lightingñ2015 Intro (85 watt/lumen) & NA & NA & 0.48 & 0.54 \\
\hline \multicolumn{5}{|l|}{ 4-Foot Fluorescent } \\
\hline F32T8 ñ Magnetic & 0.59 & 0.0 & 0.0 & 0.0 \\
\hline Electrodeless lamp & 0.0 & 0.0 & 0.03 & 0.03 \\
\hline Halogen (4024 lumen, 209 watt) & 0.28 & 0.29 & 0.29 & 0.29 \\
\hline F32T8 ñ Electronic ñ Reflector & 0.02 & 0.01 & 0.0 & 0.0 \\
\hline Solid state (2015 intro, 85 watt/lumen) & 0.0 & 0.0 & 0.52 & 0.68 \\
\hline \multicolumn{5}{|l|}{ 8-Foot Fluorescent } \\
\hline Electronic ñ Energy Saver & 0.19 & 0.65 & 0.60 & 0.0 \\
\hline Electronic ñ High Output & 0.33 & 0.34 & 0.33 & 0.34 \\
\hline Solid-state (2015 introduction, 85 watt/lumen) & NA & NA & 0.07 & 0.66 \\
\hline \multicolumn{5}{|l|}{ Two-Photon Phosphor } \\
\hline \multicolumn{5}{|l|}{ 4-Foot Fluorescent } \\
\hline Halogen & 0.28 & 0.29 & 0.28 & 0.28 \\
\hline F32T8 ñ Magnetic & 0.59 & 0.0 & 0.0 & 0.0 \\
\hline F32T8 ñ Electronic & 0.11 & 0.66 & 0.42 & 0.12 \\
\hline F32T8 ñ Electronic-Reflector & 0.02 & 0.0 & 0.0 & 0.0 \\
\hline Two-Photon Phosphor & NA & 0.05 & 0.29 & 0.59 \\
\hline \multicolumn{5}{|l|}{ 8-Foot Fluorescent } \\
\hline Efficient Magnetic ñ Energy Saver & 0.48 & 0.0 & 0.0 & 0.0 \\
\hline Electronic ñ Energy Saver & 0.19 & 0.63 & 0.56 & 0.34 \\
\hline Electronic ñ High Output & 0.33 & 0.34 & 0.31 & 0.29 \\
\hline Two-Photon Phosphor & & 0.026 & 0.13 & 0.37 \\
\hline
\end{tabular}

For FY 2003, the impact of the BTS activities in lighting controls and efficient lighting practices was assumed to be able to yield an incremental 5\% reduction in lighting energy use compared with current practice. (By incremental, the BTS activities are assumed to lead to these further savings over and above the control technologies that are now and likely to be offered by the private sector.) This assumption represents a technical potential - it is further assumed that up to

Building Equipment and Materials - 4
$80 \%$ of new commercial buildings could incorporate these technologies and that 20\% of the existing stock could be retrofitted with these systems. A time profile of penetration rates was based on the historical pattern of market penetration observed for electronic ballasts. An S-shaped penetration curve was fit to historical market shares for electronic ballasts and then applied to project future adoption of advanced lighting distribution systems and controls. (This curve indicated 


\section{Lighting R\&D}

\section{Building Equipment and Materials Decision Unit}

that $-50 \%$ of the ultimate market penetration is achieved after 9 years).

A simple spreadsheet model was developed to generate the energy savings' estimates based on these assumptions. NEMS was not used because it primarily competes different types of lighting sources - the BTS activities in this area are designed to reduce the demand for all lighting sources. Implicitly, NEMS re quires input assumptions regarding lighting demand (lumen/sq ft), but these values are fixed over the forecast horizon. BESET also cannot modify lighting demands over time.

The spreadsheet model required several key baseline inputs. Projected annual floor space additions by building type were based on Annual Energy Outlook 2001 ${ }^{(5)}$ and were taken from the BESET database. Baseline energy use/sq ft for lighting by building type was taken from PNNL's 1997 study to estimate end-use energy consumption for U.S. commercial buildings. ${ }^{(5)}$ The model was applied to the following building types: offices, retail, education, health services, assembly, and lodging.

The spreadsheet approach embodies a somewhat different methodology compared with BESET to determine the savings in existing buildings. Existing buildings in the spreadsheet model are all buildings that did not adopt the BTS-sponsored technologies during construction and thus include the current (2003) stock of buildings as well as those that will be built during the forecast period but that do not initially install these technologies.

In BESET, existing buildings primarily refer only to buildings built before the base year of the forecast horizon (e.g., pre-2003 buildings). The approach in this analysis reflects the notion that future renovations of both existing and post-2002 buildings will provide opportunities to economically install these technologies. These assumptions lead to an estimate of about 18.5 billion square feet of commercial buildings adopting these tech- nologies by 2020, with a little more than half of the adoptions occurring after the buildings were first constructed (i.e., existing buildings). This amount of floor space represents about $31 \%$ of the total floor space in the building types considered.

\section{Non-Energy Benefits:}

Helps maintain U.S. semi-conductor leadership, develops U.S. leadership in lighting technology, reduces pollution and contributes to U.S. climate change goals, improves U.S. productivity from better lighting in work environments, and responds to industry-initiated collaborative.

\section{Program Strategy (\% of budget):(1)}

- Research and Development - 90\%

- Market Transformation - 10\%

- Codes and Standards - 0\%.

\section{Sources:}

(1) FY 2002 Budget Request - Data Bucket Report for Lighting R\&D Program (internal BTS document).

(2) "The Case for a National Research Program on Semiconductor Lighting," R. Haitz and F. Kish, Hewlitt-Packard Co.; J. Tsao and J. Nelson, Sandia National Laboratories. Paper presented at the 1999 Optoel ectronics Industry Development Association forum in Washington D.C., October 6, 1999.

(3) A.D. Little. 2001. Energy Savings Potential of Solid State Lighting in General Lighting Applications, Prepared for DOE's Office of Building Technology, State and Community Programs.

(4) Annual Energy Outlook 2001. 2000. Energy Information Administration, Washington, D.C.

(5) Belzer, D.B. and L.E. Wrench. End-Use Energy Consumption Estimates for U.S. Commercial Buildings, 1992. 1997. PN NL-11514, Pacific Northwest National Laboratory, Richland, Washington. 


\section{Refrigeration \& Thermal Distribution R\&D}




\section{Program Objective: $:^{(1)}$}

This program develops and promotes the use of commerdal food display and storage technologies that use less energy and less refrigerant. Water-heating activities focus on developing low-cost, high-reliability heat pump water heater concepts. The program's HVAC delivery (e.g., duct work) technologies are intended to reduce the energy losses incurred in transferring heating or cooling from the conditioning unit(s) (e.g., heat pump, furnace, and air conditioner) to the conditioned space. The refrigerant pressure charge meter and coefficient of performance (COP) meter will enable early warning of poor operation of HVAC equipment to keep installed equipment operating at design efficiencies during the service life.

\section{Long-Term Goal:(1)}

The program's long-term goal is to reduce energy for building HVAC and refrigeration equipment over the next 15 to 20 years. Specific goals include reducing energy use for building space heating and cooling by $20 \%$ to $25 \%$ ( $22.5 \%$ assumed) and supermarket refrigeration and energy use by $10 \%$ to $20 \%$ ( $15 \%$ assumed) while reducing the level of refrigerant needed.

\section{Market Segment:}

\section{Target Market}

- Market Description:(1) Commercial refrigeration, a broad classification of building equipment that collectively consumes about 1 quad of U.S. energy annually. Supermarkets consume about onethird of the energy consumed by commercial refrigeration. Residential applications include air conditioners, heat pumps, heat pump water heaters, and thermal distribution systems associated with forced air systems.

- Size of Market:(1) Commercial refrigeration markets include about 30,000 large supermarkets and 100,000 convenience stores. Other markets include hospitals, large institutional buildings, and restaurants. Residential markets include new, single-family homes.

- Market Penetration Goal for New Buildings: $93 \%$ penetration of all commercial food sales buildings in 2020 and 99\% by 2030.

\section{Refrigeration and Thermal Distribution R\&D}

\section{Program Type:}

Equipment Efficiency

\section{Target Market:}

Refrigeration: commercial food sales in all climate zones

Heat Pump Water Heater and HVAC Distribution: residential

\section{End Uses:}

Heating, cooling, and water heating

Unit of Measurement:

Refrig.: \% end-use consumption reduction; Heat Pump Water Heater and Refrigerant Meter: efficiency/ unit; HVAC Dist: \% change in load

Modeling Tool:

Refrig.: spreadsheet HVAC Dist., Heat Pump Water Heater, and Refrig.: BESET

\section{Program Manager: \\ Esher Kweller}

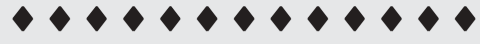

FY 2003 Benefits

Primary Energy Savings (TBtu)

\begin{tabular}{llll}
2003 & 2005 & 2010 & 2020 \\
\hline 1.7 & 7.4 & 43.6 & 202.8
\end{tabular}

Carbon Equivalent Reductions (MMTCE)

\begin{tabular}{llll}
2003 & 2005 & 2010 & 2020 \\
\hline .029 & .139 & .882 & 3.912
\end{tabular}

Consumer Cost Savings (million \$)

\begin{tabular}{llll}
2003 & 2005 & 2010 & 2020 \\
\hline 11 & 49 & 319 & 1831
\end{tabular}

Building Equipment and Materials - 7 


\section{Refrigeration and Thermal Distribution R\&D}

\section{Building Equipment and Materials Decision Unit}

\section{Methodology}

For FY 2003, four technologies were modeled: commercial refrigeration, residential HVAC distribution systems, advanced heat pump water heaters, and refrigerant pressure charge meter and COP meter.

\section{Commercial Refrigeration}

This program was modeled as an advanced supermarket refrigeration system, which would target heating, cooling, and refrigeration end-use loads in the commercial food sales sector. These end uses comprise about $66 \%$ of total building, about $67 \%$ of electric, and $61 \%$ of total natural gas end-use energy consumption. ${ }^{(2)}$

To calculate the program's energy savings, the overall reduction in end-use energy consumption was applied to the estimated consumption per square foot within food sales buildings and then was aggregated to a program total based on a forecast of square feet of food sales buildings and an estimated market penetration curve.

\section{Performance Objective:}

- Displaced Technology: Conventional refrigeration equipment in food sales buildings.

- Performance Target: Reduced energy for building HVAC and refrigeration equipment over the next 15 to 20 years, specifically reduced energy use for supermarket refrigeration and HVAC by at least 15\% while reducing refrigerant needed. For $\mathrm{FY}$ 2003, an overall $22.5 \%$ reduction in HVAC end-use energy consumption was assumed.

\section{Market Penetration:}

- Target Market: All commercial food sales buildings.

- Market Introduction: 2003; this program was assumed to accelerate the introduction of this technology into the marketplace by 10 years.

Building Equipment and Materials - 8
- Market Penetration Goal for New Buildings: $93 \%$ of all commercial food sales buildings in 2020 and $99 \%$ by 2030 .

\section{Residential HVAC Distribution Systems ñ Existing Construction}

This technology, aeroseal sealing, and other programs designed to reduce duct leakage in existing residences will reduce the energy liabilities of residential ductwork. The program's energy savings were determined by applying overall percentage reductions in heating, cooling, and ventilation to the estimated consumption per square foot within existing single-family buildings. BESET was used to perform the analysis. Ventilation consumption and savings were assumed to be included in the heating and cooling consumption and savings. The per-square-foot energy-savings' level was then aggregated to a program total based on a forecast of singlefamily building square feet and an estimated market penetration curve.

\section{Performance Objective:}

- Displaced Technology: Current duct work.

- Performance Target: For FY 2003, the following reductions in HVAC end-use energy consumption were assumed.

\begin{tabular}{|c|c|}
\hline End Use & $\begin{array}{l}\text { Percentage Reduction in Energy } \\
\text { Consumption (existing construction) }\end{array}$ \\
\hline Heating & $13.8^{*}$ \\
\hline Cooling & $6.9^{* *}$ \\
\hline \multicolumn{2}{|c|}{$\begin{array}{l}{ }^{*} \text { The seasonal heating efficiency of typical current ducts is } \\
56 \% \text { and } \sim 72 \% \text { for conventionally designed ducts with } \\
\text { R-4 duct insulation. }{ }^{(3)} \text { Single-family buildings with ducts } \\
\text { can reduce heating energy consumption by } 22.2 \% \\
(1-56 \% / 72 \%) \text {. With } ~ 50 \% \text { of existing single-family homes } \\
\text { using ducts, }(4) \text { overall per building heating savings are } \\
11.1 \%\left(22.2 \%{ }^{*} 50 \%\right) \text {. } \\
\text { ** The seasonal cooling efficiency of typical current ducts } \\
\text { is } \sim 75 \% \text { and } ~ 87 \% \text { for conventionally designed ducts with } \\
\text { R-4 duct insulation. (3) Single-family buildings with ducts } \\
\text { can reduce cooling energy consumption by } 13.8 \% \text { ( } 1 \text { - } \\
75 \% / 87 \%) \text {. With } ~ 50 \% \text { of existing single-family homes } \\
\text { using ducts, }(4) \text { overall per building heating savings are } \\
6.9 \%(13.8 \% \text { * } 50 \%) \text {. }\end{array}$} \\
\hline
\end{tabular}




\section{Refrigeration and Thermal Distribution R\&D}

\section{Building Equipment and Materials Decision Unit}

Market Penetration:

- Market Introduction: 2003; these programs were assumed to accelerate the introduction of these technologies into the marketplace by 10 years

- Market Penetration Goal: $20 \%$ of new single-family units by 2020.

Advanced Electric Heat Pump Water Heaters This technology will increase the efficiency of residential and commercial electric water heating equipment and will reduce peak energy use. This program's energy savings were determined using BESET.

\section{Performance Objective:}

- Displaced Technology: Current electric water heater technology.

- Performance Target: 2.47 energy factor.

\section{Market Penetration:}

- Target Market: Residential and commercial.

- Market Introduction: 2005, this program was assumed to accelerate the introduction of this technology into the marketplace by 10 years.

- Market Penetration Goal: $6 \%$ by 2015 and $10 \%$ by 2020 .

\section{Refrigerant Pressure Charge Meter and COP Meter}

This technology will increase the efficiency of residential and commercial space conditioning equipment and reduce peak energy use. The energy savings were determined by applying overall percentage reductions in vapor compression heating and cooling energy consumption using BESET.

\section{Performance Objective:}

- Displaced Technology: None, applied to existing and future technology.

- Performance Target: For FY 2003, the assumed reductions in HVAC end-use energy consumption are shown below.

\begin{tabular}{|l|l|}
\hline \multicolumn{1}{|c|}{ End Use } & \multicolumn{1}{|c|}{$\begin{array}{c}\text { \% Reduction in } \\
\text { Energy } \\
\text { Consumption }\end{array}$} \\
\hline Residential Heat Pump Heating & $23.9^{*}$ \\
\hline $\begin{array}{l}\text { All Residential Cooling (includes heat } \\
\text { pumps) }\end{array}$ & 23.9 \\
\hline Commercial Heat Pump Heating & $12.0^{* *}$ \\
\hline $\begin{array}{l}\text { Commercial Vapor Compression } \\
\text { Cooling (includes heat pumps and } \\
\text { excludes chillers) }\end{array}$ & 12.0 \\
\hline * This value is based on a frequency distribution of un- \\
dercharging and overcharging and an efficiency impact \\
associated with each level of undercharging and over- \\
charging. \\
http://www.proctoreng.com/checkme/technical.html. \\
$* *$ While the impact of undercharging and overcharging in \\
commercial equipment is about half that of residential \\
equipment, the frequency of undercharging and \\
overcharging is believed to be about half that in residential \\
equipment.
\end{tabular}

Market Penetration (PNNL estimates):

- Market Introduction: 2002; this program was assumed to accelerate the introduction of this technology into the marketplace by 10 years.

- Market Penetration Goals: $50 \%$ of all applicable residential units by 2020 and $90 \%$ of all applicable commercial units by 2020.

Refrigerant Pressure Charge Meter and COP Consumer Costs (PNNL estimates):

- Cost of Conventional Technology: $\$ 0.00$

- Cost of BTS Technology: $\$ 100.00$.

- Incremental Cost: $\$ 100.00$.

\section{Non-Energy Benefits:}

Reduced carbon emissions, economic benefits to private sector, reduced pollution from leaking refrigerant, and improved indoor air quality from better humidity control.

\section{Program Strategy (\% of budget): ${ }^{(1)}$}

- Research and Development - 100\%

- Market Transformation - 0\%

- Codes and Standards - 0\%. Building Equipment and Materials - 9 


\section{Refrigeration and Thermal Distribution R\&D}

Building Equipment and Materials Decision Unit

Sources:

(1) FY 2002 Budget Request - Data Bucket Report for Space Conditioning and Refrigeration: Refrigeration Program (internal BTS document).

(2) Belzer, D.B and L.E. Wrench. 1997. End-Use Consumption Estimates for U.S. Commercial Buildings, 1992. PNNL11514, Pacific Northwest National Laboratory, Richland, Washington.
(3) Brookhaven National Laboratory. 2001. Better Duct Systems for Home Heating and Cooling. BNL-68167, Vol. 4, Upton, New York, p.10.

(4) Brookhaven National Laboratory. 2001. Better Duct Systems for Home Heating and Cooling. BNL-68167, Vol. 3, Upton, New York, p.1. 


\section{Emerging \\ Technologies R\&D}




\section{Emerging Technologies R\&D}

\section{Building Equipment and Materials Decision Unit}

\section{Program Objective: ${ }^{(1)}$}

This program helps manufacturers and utilities commercialize highly efficient appliances and equipment by providing the following assistance:

- Technology procurement to bring new products to market (late developmental work), which can bridge the gap between traditional $R \& D$ and mainstream deployment.

- For emerging technology products and Energy Star products with very low market penetration, independent third-party evaluation and verification of highly efficient products using field studies and demonstrations to increase market share.

- $R \& D$ on appliances that are not covered by other programs but that offer significant energy-savings' potential.

\section{Long-Term Goals:(1)}

The program's long-term goal is to establish a track record of commercializing highly efficient products that become entrenched in the mainstream market and that become the basis for other mechanisms, such as Energy Star or minimum efficiency standards.

\section{Market Segment:}

\section{Target Market}

- Market Description: Residential and commercial building products, with emphasis on appliances and water heating.

- Size of Market: Depends on the various equipment:

- Heat Pump Water Heater: 13.6 million existing homes of the potentially 44 million home with electric resistance water heaters and $-40 \%$ of new homes. Limited, but initial market, for light commercial.

- Gas-Condensing Water Heaters: -20 million existing homes of the potential 60 million homes and $\sim 40 \%$ of new homes.

- Rooftop Air Conditioners: One of the most widely used products with greatest commercial space conditioning energy use. Over 5,260,000 tons of rooftop-air-conditioning were sold in 1998.

- Residential Can Lights: Projected incandescent can fixture sales in 2001 of 21,516,000 units.

\section{Emerging Technologies} R\&D

Program Type:

Equipment Efficiency

Target Market:

All sectors, all climate zones

\section{End Uses:}

Water heaters, lighting, dryers, and space cooling

Unit of Measurement:

Efficiency of specific equipment type

Modeling Tool:

NEMS and spreadsheets

Program Manager:

Jim Brodrick

Website:

www.eren.doe.gov/buildings/

emergingtech/index.html

FY 2003 Benefits

Primary Energy Savings (TBtu)

\begin{tabular}{llll}
2003 & 2005 & 2010 & 2020 \\
\hline 2.0 & 14.5 & 79.6 & 161.9
\end{tabular}

Carbon Equivalent Reductions (MMTCE)

\begin{tabular}{cccc}
2003 & 2005 & 2010 & 2020 \\
\hline .036 & .270 & 1.598 & 2.994
\end{tabular}

Consumer Cost Savings (million \$)

\begin{tabular}{llll}
2003 & 2005 & 2010 & 2020 \\
\hline 14 & 101 & 647 & 1452
\end{tabular}

Building Equipment and Materials - 11 


\section{Emerging Technologies R\&D}

Building Equipment and Materials Decision Unit

- Reflector Compact Fluorescent Lights (R-lamps): 125 million Parabolic/Reflector lamps sold to the residential market.

\section{Methodology}

The size of the target market is determined by the building stock, existing equipment market shares, and the turnover or replacement rate of existing equipment. The BTS technology is assumed to compete only with "new" units (or equipment sales). For new buildings, all units are considered new. For existing buildings, only units scheduled for replacement are considered eligible for the BTS technology; the BTS technology is assumed not to replace a piece of equipment unless the equipment was going to be replaced anyway.

The penetration rates for new buildings refer to the penetration into buildings built in 2003 and beyond. The penetration into existing buildings is the penetration into all buildings built before 2003 .

All of the programs, except lighting, were modeled with a modified version of NEMS. The lighting programs were modeled in BESET. The NEMS key inputs and outputs are described in a separate section below.

\section{Heat Pump Water Heater}

- Market Introduction: 2005; these programs were assumed to accelerate the introduction of these technologies into the marketplace by 10 years.

- Performance Target: 2.0 energy factor (demonstrated).

- Penetration Target: 6\% by 2015 (may want a more aggressive sales target such as 10\%; building codes are expected to help by excluding electric resistance water heating, as will be case in California).
- Installed Cost: I nitial installation cost of $\$ 700$, decreasing to $\$ 650$ in 2010.

- Lifetime: 10 years.

Gas-Condensing Water Heater

- Market Introduction: 2002; these programs were assumed to accel erate the introduction of these technologies into the marketplace by 10 years.

- Performance Target: Energy factor of 0.80.

- Penetration Target: 9\% to $10 \%$ by 2020.

- Installed Cost: Incremental cost of \$150 to $\$ 200$ over conventional technology. Estimated from a study that references a phone call with A.D. Little and gives a price of $\$ 700 .{ }^{(3)}$

- Lifetime: 10 years.

\section{Rooftop Air Conditioning}

- Market Introduction: 2004; The NAS (acceleration-to-market) methodology was not applied to this technology because it was determined that the impact would be negligible. Given that the technology has only modest penetration (10\%) by 2020 and only a few percent by 2010, the NAS methdology would not have significant impact over the analysis period.

- Performance Target: An efficiency increase from 10.3 to 11.0 energy efficiency ratio for 65 to $135 \mathrm{kBtu} / \mathrm{hr}$ and from 9.7 to 10.8 for 135 to $240 \mathrm{kBtu} / \mathrm{hr}$.

- Penetration Target: 10\% of sales in 2020.

- Lifetime: 15 years.

\section{Residential Can Lights}

- Market Introduction: 2003; these programs were assumed to accelerate the introduction of these technologies into the marketplace by 10 years.

- Performance Target: Assumed efficacy of 51.3 lumen/watt. Actual program requirements should be similar to Energy Star, as the following table shows. However, be- 


\section{Emerging Technologies R\&D}

\section{Building Equipment and Materials Decision Unit}

cause incandescent lamps also have lower efficacies, the ratio between Energy Star compact fluorescent lamps and all incandescents was assumed to be the same regardless of type. See the Energy Star compact fluorescent lamp writeup.

- Penetration Target: $2.1 \%$ of all incandescents (30\% of recessed can lights by 2008 ). The $30 \%$ penetration rate was assumed to occur across all fixtures in a

\begin{tabular}{|l|c|}
\hline \multicolumn{1}{|c|}{$\begin{array}{c}\text { Lamp Power (watts) and } \\
\text { Configuration }\end{array}$} & $\begin{array}{c}\text { Minimum Efficacy: } \\
\text { Lumens/watt }\end{array}$ \\
\hline Reflector Lamp: & 33 \\
Lamp power $<20$ & 40 \\
Lamp power $>=20$ & \\
\hline * Based on initial lumen date. & \\
\hline
\end{tabular}

home; that is, a home was assumed to have either all compact fluorescent light cans or all incandescent cans. Can lights represent about $7 \%$ of all incandescent fixtures $(2.1 \%$ $=30 \% * 7 \%$ ). In $2020,7 \%$ of the incandescent market will be recessed can lights. ${ }^{(2)}$

- Lifetime: 30 years.

\section{R-Lamps}

- Market Introduction: These programs were assumed to accelerate the introduction of these technologies into the marketplace by 4 years.

- Performance Target: Assumed efficacy of 51.3 lumen/watt. Actual program requirements should be similar to Energy Star, as the table below shows. However, because incandescent lamps also have lower efficacies, the ratio between Energy Star compact fluorescent lamps and all incandescents were assumed to be the

\begin{tabular}{|l|c|}
\hline \multicolumn{1}{|c|}{$\begin{array}{c}\text { Lamp Power (watts) and } \\
\text { Configuration }\end{array}$} & $\begin{array}{c}\text { Minimum Efficacy: } \\
\text { Lumens/watt }\end{array}$ \\
\hline Reflector Lamp: & 33 \\
Lamp power $<20$ & 40 \\
Lamp power >=20 & \\
\hline * Based on initial lumen date. & \\
\hline
\end{tabular}

same regardless of type. See the Energy Star compact fluorescent lamp writeup.

- Penetration Target: $0.87 \%$ of all incandescents (10\% of reflector lights by 2008 ). An $8.47 \%$ sales fraction will result in a longterm installed base of $27 \%$, which has consumption about equal to a $10 \%$ installed base in the most energy-intensive fixtures $(0.87 \%=8.47 \% * 10.3 \%)$. This penetration rate is based on a 4-year reflector compact fluorescent lamp life and a 1-year incandescent life. (The 1-year life for incandescents to about a factor of 2 high; however, integer life inputs are required for BESET. While the net energy effect is about the same, the investment numbers will require adjustment.) In 2008, $10.3 \%$ of the incandescent market will be reflector lights. ${ }^{(4)}$

- Installed Cost: \$14/compact fluorescent Iamp Reflector Lamp.

- Lifetime: 8,000 hours (4 years for BESET).

\section{NEMS Model Inputs and Outputs}

The two programs related to water heaters were modeled with the NEMS residential model. Table 1 shows the NEMS inputs for electric water heaters, with the BTS-sponsored technology input assumptions highlighted. While the equipment types are not

\begin{tabular}{|c|c|c|c|c|c|c|}
\hline \multicolumn{7}{|c|}{$\begin{array}{l}\text { Table 1. NEMS Residential Model Inputs for } \\
\text { High-Efficiency Heat Pump Water Heater }\end{array}$} \\
\hline $\begin{array}{l}\text { Equip. } \\
\text { Type }\end{array}$ & $\begin{array}{l}\text { Last } \\
\text { Year } \\
\text { Avail } \\
\end{array}$ & $\begin{array}{c}\text { Energy } \\
\text { Factor } \\
\end{array}$ & $\begin{array}{c}\text { Installed } \\
\text { Cost }\end{array}$ & $\begin{array}{c}\text { Logit } \\
\text { Model } \\
\text { Para: } \\
\text { Beta1 }\end{array}$ & $\begin{array}{l}\text { Logit Model } \\
\text { Para: Beta2 }\end{array}$ & $\begin{array}{c}\text { Implied } \\
\text { Discount } \\
\text { Rate }\end{array}$ \\
\hline 7 & 2020 & 0.86 & $\$ 350$ & -0.01619 & -0.01952 & 0.83 \\
\hline 8 & 2020 & 0.88 & $\$ 350$ & -0.01619 & -0.01952 & 0.83 \\
\hline 9 & 2020 & 0.95 & $\$ 575$ & -0.01619 & -0.01952 & 0.83 \\
\hline 10 & 2020 & 2.60 & $\$ 1,025$ & -0.01619 & -0.01952 & 0.83 \\
\hline 11 & 2020 & 2.00 & $\$ 2,600$ & -0.01619 & -0.01952 & 0.83 \\
\hline 12 & 2020 & 0.89 & $\$ 350$ & -0.01619 & -0.01952 & 0.83 \\
\hline 13 & 2020 & 0.96 & $\$ 475$ & -0.01619 & -0.01952 & 0.83 \\
\hline 14 & 2020 & 2.47 & $\$ 700$ & -0.01619 & -0.01952 & 0.83 \\
\hline 15 & 2020 & 0.90 & $\$ 400$ & -0.01619 & -0.01952 & 0.83 \\
\hline 16 & 2020 & 0.96 & $\$ 425$ & -0.01619 & -0.01952 & 0.83 \\
\hline 17 & 2020 & 2.47 & $\$ 650$ & -0.01619 & -0.01952 & 0.83 \\
\hline
\end{tabular}




\section{Emerging Technologies R\&D}

\section{Building Equipment and Materials Decision Unit}

identified by name, the table illustrates the range of technologies competed within NEMS. Note that EIA assumes several levels of resistance water heaters with energy factors ranging from 0.86 to 0.96 . The very high-efficiency water heaters in the EIA base case are very costly.

Table 2 shows the penetration of the sales of BTS-sponsored heat pump water heater for new homes and for replacement of existing electric water heaters. In the first few years of the programs, NEMS projected the market share to be $\sim 1 \%$. After the assumed $\$ 50$ cost reduction in 2010, the market share increases to a little more than 3\%. These low shares are

\begin{tabular}{|c|c|c|c|c|}
\hline \multicolumn{5}{|c|}{$\begin{array}{c}\text { Table 2. NEMS-Generated Penetration Rates for } \\
\text { High-Efficiency Heat Pump Water Heaters }\end{array}$} \\
\hline Year & $\begin{array}{c}\text { In New } \\
\text { Housing } \\
\text { Units }\end{array}$ & $\begin{array}{c}\text { Fraction of } \\
\text { Electric } \\
\text { Water } \\
\text { Heater }\end{array}$ & $\begin{array}{c}\text { Fraction of } \\
\text { Electric } \\
\text { Wnits } \\
\text { Weater } \\
\text { Heater }\end{array}$ \\
\hline 2005 & 7,325 & 0.011 & 26,435 & 0.012 \\
\hline 2006 & 7,374 & 0.011 & 27,020 & 0.012 \\
\hline 2007 & 7,461 & 0.011 & 27,343 & 0.012 \\
\hline 2008 & 7,345 & 0.011 & 27,507 & 0.012 \\
\hline 2009 & 7,142 & 0.010 & 27,861 & 0.012 \\
\hline 2010 & 22,773 & 0.033 & 90,881 & 0.037 \\
\hline 2015 & 18,268 & 0.026 & 73,876 & 0.029 \\
\hline 2020 & 17,515 & 0.025 & 74,509 & 0.028 \\
\hline
\end{tabular}

largely attributed to the high discount rate used in NEMS. As the last column of Table 1 shows, the value of the discount rate for electric water heaters was assumed to be over $80 \%$. Thus, in spite of the performance differential for the heat pump unit, the higher first cost was assumed to be large a barrier in promoting widespread adoption of this technology.

NEMS has no technology diffusion algorithm that will produce a gradual increase in market shares without any change in cost and performance. Therefore, the market shares from 2005 through 2009 are virtually the same. It

Building Equipment and Materials - 14 can be argued that the model provides an average penetration that yields the same level of total sales as would an S-shaped penetration curve. The market shares dedine in the out years, 2015 and 2020, because of the cost reductions assumed for the resistance water heater in the base case.

Table 3 shows the NEMS inputs for gas water heaters, with the input assumptions highlighted for the BTS-sponsored technology. Again, while the equipment types are not identified by name, the table illustrates the range of technologies competed within NEMS. Note that several levels of gas water heaters (noncondensing) with energy factors ranging from 0.54 to 0.70 are assumed. With an energy factor of 0.86 , the gas-condensing water heaters in the base case are very costly.

Table 4 shows the penetration of the BTSsponsored technology for sales to new homes and for replacement of existing gas water heaters. In the first few years of the programs, NEMS projected the market share to be $\sim 2 \%$. After the assumed $\$ 25$ cost reduction in 2010, the market share increases to $-6 \%$ in new homes and over $7 \%$ for replacement units. These low shares are largely attributed to the high discount rate used in NEMS.

The behavior of the technology's predicted shares is similar to that of the heat pump water heater. The shares fall slightly over the full forecast period as the cost and performance of competing (noncondensing) water heaters improve.

\section{Non-Energy Benefits:}

Water conservation devices and high-efficiency appliances save water and waste water. These benefits are being better characterized and will be used to justify water/waste water utilities implementing demand-side management programs. 


\section{Emerging Technologies R\&D}

Building Equipment and Materials Decision Unit

\begin{tabular}{|l|c|c|c|c|c|c|}
\hline \multicolumn{7}{|c|}{ Table 3. NEM Residential Model Inputs for Gas-Condensing Water Heaters* } \\
\hline $\begin{array}{l}\text { Equipment } \\
\text { Type }\end{array}$ & $\begin{array}{c}\text { Last Year } \\
\text { Available }\end{array}$ & Efficiency & Installed Cost & $\begin{array}{c}\text { Logit Model } \\
\text { Para: Beta1 }\end{array}$ & $\begin{array}{c}\text { Logit Model } \\
\text { Para: Beta2 }\end{array}$ & $\begin{array}{c}\text { Implied } \\
\text { Discount Rate }\end{array}$ \\
\hline 1 & 2020 & 0.54 & $\$ 340$ & -0.05393 & -0.1136 & 0.47 \\
\hline 2 & 2020 & 0.58 & $\$ 370$ & -0.05393 & -0.1136 & 0.47 \\
\hline 3 & 2004 & 0.6 & $\$ 400$ & -0.05393 & -0.1136 & 0.47 \\
\hline 3 & 2020 & 0.6 & $\$ 375$ & -0.05393 & -0.1136 & 0.47 \\
\hline 4 & 2004 & 0.86 & $\$ 2,360$ & -0.05393 & -0.1136 & 0.47 \\
\hline 4 & 2014 & 0.86 & $\$ 2,000$ & -0.05393 & -0.1136 & 0.47 \\
\hline 4 & 2020 & 0.86 & $\$ 1,800$ & -0.05393 & -0.1136 & 0.47 \\
\hline 5 & 2014 & 0.63 & $\$ 450$ & -0.05393 & -0.1136 & 0.47 \\
\hline 5 & 2020 & 0.63 & $\$ 425$ & -0.05393 & -0.1136 & 0.47 \\
\hline 6 & 2020 & 0.7 & $\$ 500$ & -0.05393 & -0.1136 & 0.47 \\
\hline 26 & 2020 & 0.8 & $\$ 500$ & -0.05393 & -0.1136 & 0.47 \\
\hline 27 & 2020 & 0.8 & $\$ 475$ & -0.05393 & -0.1136 & 0.47 \\
\hline Highlighted cells indicate BTS programs. ${ }^{*}$ Lower-cost model introduced in 2010. & \\
\hline
\end{tabular}

\begin{tabular}{|c|c|c|c|c|}
\hline \multicolumn{5}{|c|}{ Table 4. Sales Penetration for Gas-Condensing } \\
Water Heaters \\
\hline Year & $\begin{array}{c}\text { In New } \\
\text { Housing } \\
\text { Units }\end{array}$ & $\begin{array}{c}\text { Fraction of } \\
\text { Gas Water } \\
\text { Heater }\end{array}$ & $\begin{array}{c}\text { Replace. } \\
\text { Units }\end{array}$ & $\begin{array}{c}\text { Fraction of } \\
\text { Gas Water } \\
\text { Heater }\end{array}$ \\
\hline 2003 & 19,424 & 0.019 & 60,336 & 0.019 \\
\hline 2004 & 19,481 & 0.019 & 62,811 & 0.020 \\
\hline 2005 & 15,445 & 0.014 & 48,305 & 0.015 \\
\hline 2010 & 60,035 & 0.056 & 234,092 & 0.071 \\
\hline 2015 & 55,912 & 0.054 & 233,794 & 0.068 \\
\hline 2020 & 46,945 & 0.046 & 230,349 & 0.059 \\
\hline
\end{tabular}

\section{Sources:}

(1) FY 2002 Budget Request - Data Bucket Report for Appliances and Emerging Technology Program (internal BTS document).

(2) Estimated from http://enduse.lbl.gov// nfo/ LBNL-39102.pdf, p.19.

(3) Gordon, K.L. and M.R. Ledbetter., 2001. Technology Procurement Screening Study. Pacific Northwest National Laboratory, Richland, Washington.

(4) The Freedonia Group, Inc., 1999, Lamps in the United States to 2003 - Introduction, Executive Summary, Market Environment, Supply and Demand, Incandescent Lamps, Electrical Discharge and Lamp Markets, Cleveland, Ohio. 


\section{Building Envelope R\&D: Windows}




\section{Building Envelope R\&D: Windows}

\section{Building Equipment and Materials Decision Unit}

\section{Program Objective:(1)}

The program's objective is to reward the marketplace for industry's investments in researching, developing, and deploying energy-efficient windows. Because the area is less suited to national standards and has a growing international market, significant investments are needed to establish a technical basis for performance standards recognized for scientific excellence. On this basis, the program helps develop the credible rating, certification programs,and design tools to develop and apply efficient windows. The program also develops viable technologies, electrochromics, and durable spectrally selective glazing from competing producers.

\section{Long-Term Goals: ${ }^{(1)}$}

- National: Change windows from net energy loss to net energy benefits across the United States.

- Current Energy Losses: 3.8 quad.

- Environmental Impact: 76 MMtons carbon/yr.

- Industry: Strengthen market position of U.S. industry in global markets.

- Owners: Provide cost-effective savings with comfort, productivity, and amenity.

\section{Methodology}

The general approach for calculating energy savings for building envelope programs involved simulating the effect of an envelope technology using the Federal Energy Decision System (FEDS)(2) model for many different building types, sizes, vintages, and locations and then using NEMS to calculate national impacts. The heating and cooling loads were calculated for each building with and without evaluating the envelope technology. The heating and cooling load changes were then used to modify the heating and cooling envelope factors in NEMS.

These factors were input as a vector for each building type and census region; these vectors captured both the thermal impact and the expected market penetration by year. Market penetration estimates were based on input from the DOE Program Manager or representative. Specific program inputs are characterized below.

\section{Building Envelope R\&D: Windows}

\section{Program Type:}

Envelope

Target Market:

All sectors in all climate zones

\section{End Uses:}

Windows

Unit of Measurement:

$\%$ change in load (based on change in u-factor and shading coefficient)

Modeling Tool:

NEMS envelope

Program Manager:

Sam Taylor

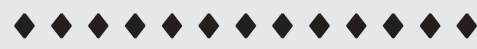

FY 2003 Benefits

Primary Energy Savings (TBtu)

\begin{tabular}{llll}
2003 & 2005 & 2010 & 2020 \\
\hline 6.9 & 15.7 & 81.7 & 554.7
\end{tabular}

Carbon Equivalent Reductions (MMTCE)

\begin{tabular}{llll}
2003 & 2005 & 2010 & 2020 \\
\hline 1.013 & .282 & 1.534 & 10.138
\end{tabular}

Consumer Cost Savings (million \$)

\begin{tabular}{llll}
2003 & 2005 & 2010 & 2020 \\
\hline 46 & 106 & 575 & 4317
\end{tabular}

Building Equipment and Materials - 17 


\section{Building Envelope R\&D: Windows}

Building Equipment and Materials Decision Unit

\section{Electrochromic Windows}

This program develops commercially viable advanced electrochromic windows using competing producers. With a focus on el ectrochromic research, the program's objective is to reward the marketplace for industry's investments in researching, developing, and deploying energy-efficient windows.

\section{Market Segment:}

\section{Performance Objective:}

- Displaced Technology: Conventional double-glazed, low-emissivity windows.

- Performance Target: Reduce unwanted heat gains and losses and perimeter lighting. Estimated savings per building were determined by simulating all commercial building types in all dimate zones. NEMS was used to determine national impacts.

- Performance Parameters: See the table below.

\begin{tabular}{|l|l|l|}
\hline \multicolumn{1}{|c|}{ Parameter } & \multicolumn{1}{c|}{ Value } & \multicolumn{1}{c|}{ Units } \\
\hline $\begin{array}{l}\text { Maximum Shading } \\
\text { Coefficient }\end{array}$ & $\begin{array}{l}0.4 \\
\text { (heating) }\end{array}$ & NA \\
\hline $\begin{array}{l}\text { Minimum Shading } \\
\text { Coefficient }\end{array}$ & $\begin{array}{l}0.1 \\
\text { (cooling) }\end{array}$ & NA \\
\hline U-value & 0.25 & $\mathrm{Btu} / \mathrm{h} \bullet \mathrm{ft}^{2} \mathrm{~F}$ \\
\hline Lighting Reduction & 30 & $\%$ of lighting energy \\
\hline
\end{tabular}

\section{Target Market}

- Market Description:(1) New and existing commercial building types in all climate zones.

- Size of Market: $\sim 500$ million square feet of windows for commercial buildings.

- Market Introduction: The program began before 1977; market introduction is targeted for the end of FY 2002 for electrochromic windows in commercial applications. This program was assumed to accelerate the introduction of this technology into the marketplace by 10 years.
- Market Penetration Goal: See the following table.

Program/Technology Consumer Costs:

- Incremental Cost of BTS Technology: $+\$ 5 / \mathrm{sq} \mathrm{ft}$ for electrochromic windows compared with conventional, doubleglazed, low-emissivity windows.

\begin{tabular}{|l|l|c|c|c|c|}
\hline \multicolumn{6}{|c|}{ Rate of Penetration (\% of annual sales) } \\
\hline $\begin{array}{c}\text { Building } \\
\text { Type }\end{array}$ & Vintage & Region & $\mathbf{2 0 0 5}$ & $\mathbf{2 0 2 0}$ & $\mathbf{2 0 3 0}$ \\
\hline $\begin{array}{l}\text { All } \\
\text { Commercial }\end{array}$ & New & All & 2 & 20 & 50 \\
\hline $\begin{array}{l}\text { All } \\
\text { Commercial }\end{array}$ & Existing & All & 1.8 & 17.2 & 43.2 \\
\hline
\end{tabular}

\section{Superwindows}

The program is developing commercially viable advanced technologies from competing producers and providing research support to Energy Star and Efficient Window Collaborative programs. One program objective is to double the average energy efficiency of windows sold and establish universal NFRC (National Fenestration Rating Council) ratings based on credible International Standards Organization (ISO) standards.

\section{Market Segment:}

\section{Performance Objective:}

- Displaced Technology: Conventional double-glazed, low-emissivity windows with a U-value of $0.357 \mathrm{Btu} / \mathrm{h} \cdot \mathrm{ft}^{2}{ }^{\circ} \mathrm{F}$ and a shading coefficient of .52 .

- Performance Target: Reduce unwanted heat gains and losses. The estimated savings per building was determined by simulating residential buildings in all climate zones. National impacts were determined using NEMS.

- Performance Parameters: Two superwindow technologies were used: northern superwindows in heating dominated climates (heating-degree days 


\section{Building Envelope R\&D: Windows}

Building Equipment and Materials Decision Unit

\begin{tabular}{|c|l|l|l|}
\hline Window & \multicolumn{1}{|c|}{ Parameter } & \multicolumn{1}{c|}{ Value } & \multicolumn{1}{c|}{ Units } \\
\hline Northern Superwindow & Shading Coefficient & 0.7 (heating season) & $\mathrm{NA}$ \\
& & 0.3 (cooling season) & \\
\cline { 2 - 4 } & U-value & 0.1 & $\mathrm{Btu} / \mathrm{h} \bullet \mathrm{ft}^{2 \circ} \mathrm{F}$ \\
\hline \multirow{2}{*}{ Southern Superwindow } & Shading Coefficient & 0.15 (all seasons) & $\mathrm{NA}$ \\
\cline { 2 - 4 } & U-value & 0.2 & $\mathrm{Btu} / \mathrm{h} \bullet \mathrm{ft}^{2} \mathrm{o}^{\mathrm{F}}$ \\
\hline
\end{tabular}

>4500) and southern superwindows in cooling dominated climates (heating-degree days $<4500$ ). See the following table.

\section{Target Market}

- Market Description:(1) New and existing residential units in all climate zones.

- Size of Market:(1) About 55 million manufactured units sold each year for residential and light commercial.

- Market Introduction: The program began before 1977; market introduction was 1999 for advanced window collaborative and advanced spectrally selective glazing. This program was assumed to accel erate the introduction of this technology into the marketplace by 10 years.

- Market Penetration Goal: See the table below.

\begin{tabular}{|c|l|c|c|c|c|}
\hline \multicolumn{6}{|c|}{ Rate of Penetration (\% of annual sales) } \\
\hline $\begin{array}{c}\text { Building } \\
\text { Type }\end{array}$ & Vintage & Region & $\mathbf{2 0 0 5}$ & $\mathbf{2 0 2 0}$ & $\mathbf{2 0 3 0}$ \\
\hline Residential & New & All & 3 & 65 & 85 \\
\hline Residential & Existing & All? & 1.5 & 33 & 43.2 \\
\hline
\end{tabular}

Program/Technology Consumer Costs:

- Incremental Cost of BTS Technology: $+\$ 6 / \mathrm{sq} \mathrm{ft}$ in $2005,+\$ 4 / \mathrm{sq} \mathrm{ft}$ in 2020 , and $\$ 3 /$ sq ft in 2030 for advanced windows over conventional double-glazed, low-emissivity windows.

\section{Non-Energy Benefits:(1)}

Could reduce both utility and building peak loads, which could reduce HVAC requirements and thus, lower first costs, offsetting increased high-performance glazing costs. Other benefits include environmental, particularly from reduced utility peak loads, comfort, and aesthetics.

\section{Sources:}

(1) FY 2002 Budget Request - Data Bucket Report for Building Envel ope: Windows Program (internal BTS document). 


\section{Building Envelope R\&D: Thermal Insulation and Building Materials}




\section{Building Envelope R\&D: Thermal Insulation and Building Materials}

\section{Building Equipment and Materials Decision Unit}

\section{Program Objective: $:^{(1)}$}

This program improves envelope performance through advanced technology and increased understanding of the basic processes governing envelope performance. Building envelopes, which influence electric lighting requirements, are the primary factor governing buildings' heating, cooling, and ventilation requirements. Because building envelopes impact $53 \%$ of building energy use, substantial energy can be saved by improving the materials, components, and systems that make up building envelopes.

This program performs research on energy-efficient, sustainable, low-cost, and super-smart thermal insulation and building envelope materials and structures. The program develops laboratory, analytical, and field experiments and methodologies to characterize tools and testing for new or improved materials and systems. The program also provides accurate evaluation procedures.

\section{Long-Term Goal:(1)}

The program's long-term goal is to develop new building materials and systems that are cost-competitive for their application and are as environmentally benign and sustainable as possible. Another long-term goal involves developing a fundamental understanding of heat, air, and moisture transfer through building envelopes and insulation materials and applying the results to develop construction technologies to increase building energy efficiency.

\section{Market Segment:}

\section{Performance Objective:}

- Displaced Technology: Conventional wall and roof insulation and framing.

- Performance Target: Reduce unwanted heat gains and losses and perimeter lighting. Estimated savings per building were determined by simulating food sales, mercantile and service, warehouse, and other commercial building types and single-family residential buildings in all climate zones. National impacts were determined by NEMS.

- Performance Parameters: See the table below.

\section{Building Envelope R\&D:} Thermal Insulation and Building Materials

Program Type:

Envelope

Target Market:

All sectors in all climate zones

\section{End Uses:}

Roofs and insulation

Unit of Measurement:

$\%$ change in load (based on change in envelope component uvalue)

Modeling Tool:

NEMS envelope

Program Manager:

Arun Vohra

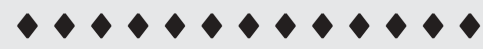

FY 2003 Benefits

Primary Energy Savings (TBtu)

\begin{tabular}{llll}
2003 & 2005 & 2010 & 2020 \\
\hline 0.0 & .09 & 9.0 & 62.1
\end{tabular}

Carbon Equivalent Reductions (MMTCE)

\begin{tabular}{llll}
2003 & 2005 & 2010 & 2020 \\
\hline .000 & .013 & .136 & .947
\end{tabular}

Consumer Cost Savings (million \$)

\begin{tabular}{llll}
2003 & 2005 & 2010 & 2020 \\
\hline 0 & 6 & 61 & 419
\end{tabular}

Building Equipment and Materials - 21 


\section{Building Envelope R\&D: Thermal Insulation and Building Materials}

Building Equipment and Materials Decision Unit

\begin{tabular}{|l|l|c|}
\hline Parameter & Value & Units \\
\hline r-value & 36 & $\mathrm{Btu} / \mathrm{h} \bullet \mathrm{ft}^{2}{ }^{\circ} \mathrm{F}$ \\
\hline
\end{tabular}

\section{Target Market}

- Market Description:(1) This program involves developing materials and building envelope structures that can be used in new residential and commercial buildings. Certain elements of the program also focus on retrofit strategies. The program activities are independent of region and household income.

- Size of Market:(1) All new and retrofit residential and commercial construction and all building categories.

\section{Methodology}

The general approach to calculating energy savings for building envelope programs involved simulating the effect of an envelope technology using the Federal Energy Decision System (FEDS)(2) model for many different building types, sizes, vintages, and locations and then using NEMS to calculate national impacts. The heating and cooling loads were calculated for each building with and without evaluating the envelope technology. The changes in the heating and cooling loads were then used to modify the heating and cooling envelope factors used in NEMS.

These factors were input as a vector for each building type and census region; these vectors captured both the thermal impact and the expected market penetration by year. Market penetration estimates were based on input from the DOE Program Manager or representative. Specific program inputs are characterized below.

\section{Quick Fill Walls}

Description: This technology involves applying environmental wall-insulating techniques.

Building Equipment and Materials - 22
Performance Objective:

- Displaced Technology: Conventional wall insulation and framing.

- Performance Target: Reduce unwanted heat gains and losses and perimeter lighting. Estimated savings per building were determined by simulating food sales, mercantile and service, warehouse, and other commercial building types and singlefamily residential buildings in all dimate zones. NEMS was used to determine national impacts.

- Performance Parameters: See the table below.

\begin{tabular}{|l|l|c|}
\hline Parameter & Value & Units \\
\hline r-value & 36 & $\mathrm{Btu} / \mathrm{h} \bullet \mathrm{ft}^{2} \mathrm{~F}$ \\
\hline
\end{tabular}

Market Penetration:

- Target Market: Selected commercial and residential buildings in all climate zones.

- Market Introduction: 2004; this activity was assumed not to occur without DOE funding; therefore, the NAS methodology was not applied.

- Market Penetration Goal: See the table below.

\begin{tabular}{|l|c|c|c|c|}
\hline \multicolumn{5}{|c|}{ Rate of Penetration (\% of annual sales) } \\
\hline \multicolumn{1}{|c|}{ Building Type } & Vintage & Region & $\mathbf{2 0 1 0}$ & $\mathbf{2 0 3 0}$ \\
\hline $\begin{array}{l}\text { Food Sales, } \\
\text { Mercantile and } \\
\begin{array}{l}\text { Service, Ware- } \\
\text { house, and Other }\end{array}\end{array}$ & New & All & 1.5 & 7.6 \\
\hline Residential & New & All & 2.3 & 11.5 \\
\hline
\end{tabular}

R30 Insulation/30-Year Life Roofs Description: This technology involves applying advanced roofing techniques.

Performance Objective:

- Displaced Technology: Conventional roof insulation.

- Unit of Measurement: Cooling and heating load reductions for commercial buildings. 


\section{Building Envelope R\&D: Thermal Insulation and Building Materials}

\section{Building Equipment and Materials Decision Unit}

- Performance Target: Reduce unwanted heat gains and losses. Estimated savings per building were determined by simulating all commercial building types in all climate zones. National impacts were determined in NEMS.

- Performance Parameters: See the table below.

\begin{tabular}{|l|l|c|}
\hline Parameter & Value & Units \\
\hline r-value & 30 & $\mathrm{Btu} / \mathrm{h} \cdot \mathrm{ft}^{2}{ }^{\circ} \mathrm{F}$ \\
\hline
\end{tabular}

Market Penetration:

- Target Market: All sectors in all climate zones.

- Market Introduction: 2010; this activity was assumed not to occur without DOE funding; therefore, the NAS methodology was not applied.

- Market Penetration Goal: See the table below.

\begin{tabular}{|c|l|c|c|c|}
\hline \multicolumn{5}{|c|}{ Rate of Penetration (\% of annual sales) } \\
\hline Building Type & Vintage & Region & $\mathbf{2 0 1 0}$ & $\mathbf{2 0 3 0}$ \\
\hline All Commercial & New & All & 0.6 & 50.5 \\
\hline All Commercial & Existing & All & 0.4 & 30.2 \\
\hline
\end{tabular}

\section{Moisture/Wet Insulation}

\section{Performance Objective:}

- Performance Target: Reduce unwanted heat gains and losses by $10 \%$. National impacts were determined in NEMS.

\section{Market Penetration:}

- Target Market: All residential buildings in all climate zones.

- Market Introduction: 2004; this activity was assumed not to occur without
DOE funding; therefore, the NAS methodology was not applied.

- Market Penetration Goal: See the table below.

\begin{tabular}{|c|l|c|c|c|l|}
\hline \multicolumn{6}{|c|}{ Rate of Penetration (\% of annual sales) } \\
\hline $\begin{array}{c}\text { Building } \\
\text { Type }\end{array}$ & Vintage & Region & $\mathbf{2 0 0 5}$ & $\mathbf{2 0 2 0}$ & $\mathbf{2 0 3 0}$ \\
\hline Residential & New & All & 3 & 65 & 85 \\
\hline Residential & Existing & All & 1.5 & 33 & 43.2 \\
\hline
\end{tabular}

\section{Non-Energy Benefits:(1)}

Reduced construction and demolition waste; use of natural, recyded, and byproduct materials; reduced $\mathrm{CO}_{2}$ emissions from improved energy efficiency; increased housing affordability from reduced energy consumption; improved comfort and indoor air quality from more moisture tolerant designs and controls; and increased global competitiveness of U.S. industry.

\section{Program Strategy (\% of budget):(1)}

- Research and Development - 95\%

- Market Transformation - 0\%

- Codes and Standards - 5\%.

\section{Sources:}

(1) FY 2002 Budget Request - Data Bucket Report for Building Envelope: Thermal Insulation and Buildings Materials Program (internal BTS document).

(2) Pacific Northwest National Laboratory. 1998. Facility Energy Decision System User's Guide, Release 4.0. PNNL-10542, Rev. 2, Richland, Washington. 


\section{Design Strategies and Assistance}




\section{Design Strategies and Assistance}

\section{Building Equipment and Materials Decision Unit}

\section{Program Objective:(1)}

The Design Strategies and Assistance program researches the interrelationship of energy systems and buildings energy performance, develops various building analysis tools to more accurately model energy use in new and existing buildings, and provides recommendations and strategies to cost effectively lower energy use and improve building performance. The program focuses on whole-building software tools for evaluating energy efficiency and renewable energy. The program also focuses on nonsoftware solutions such as improved standards, guidelines, and performance measurements, all of which bring about excellence in designing new buildings.

\section{Long-Term Goal:(1)}

The program's long-term goal is to improve energy designs for all building types through a suite of widely used analytical tools and guidance documents.

\section{Market Segment:}

Performance Objective:(2)

- Displaced Technology: Conventional design/ building practice.

- Performance Target: By 2020 reduce heating and cooling loads by $50 \%$ in new construction and use energy analysis tools in $60 \%$ of new commercial buildings.

\section{Target Market: ${ }^{(2)}$}

- Market Description: All new commercial and retrofit construction (particularly buildings with energy-use intensities $>50 \%$ of the average energyuse intensity).

- Size of Market: New assembly, education, food service, food sales, health care, lodging, mercantile and service, and office buildings for the commercial sector.

- Market Introduction: 1996; this activity was assumed not to occur with DOE funding; therefore, the NAS methodology was not applied.

- Market Penetration Goal: Penetrate $70 \%$ of new commercial and multifamily construction by 2020 in combination with the Commercial Buildings $R \& D$ program.

\section{Design Strategies and Assistance}

\section{Program Type:}

Whole Building

\section{Target Market:}

Single-family and manufactured homeowners with $>\$ 50,000 / y r$ income, all multifamily and commercial sector and industrial offices in all climate zones

\section{End Uses:}

All end uses and all fuel types

Unit of Measurement:

$\%$ change in load

Modeling Tool:

BESET

Program Manager:

Dru Crawley

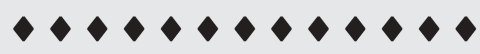

FY 2003 Benefits

Primary Energy Savings (TBtu)

\begin{tabular}{cccc}
2003 & 2005 & 2010 & 2020 \\
\hline 0.70 & 3.00 & 16.2 & 53.2
\end{tabular}

Carbon Equivalent Reductions (MMTCE)

\begin{tabular}{cccc}
2003 & 2005 & 2010 & 2020 \\
\hline .011 & 0.54 & .309 & .971
\end{tabular}

Consumer Cost Savings (million \$)

\begin{tabular}{llll}
2003 & 2005 & 2010 & 2020 \\
\hline 4 & 18 & 103 & 387
\end{tabular}

Building Equipment and Materials - 25 


\section{Design Strategies and Assistance}

Building Equipment and Materials Decision Unit

\section{Methodology}

Energy savings for this program were estimated using BESET. To simulate historical penetration rate patterns, penetration rates for all years before 2030 were calculated based on diffusion models, or "S curves" based on historical data. The diffusion models require only the maximum penetration in the final year and the year of entry into the market. Energy technology diffusion curves were estimated from historical data, and generic curves applicable to future products were developed.

\section{Non-Energy Benefits:(1)}

Improved indoor environmental quality such as thermal comfort and ventilation adequacy and improved indoor air quality, fire safety, and overall environmental sustainability (i.e., Green Buildings).

\section{Program Strategy (\% of budget): ${ }^{(1)}$}

- Research and Development - 90\%

- Market Transformation - $10 \%$

- Codes and Standards - 0\%.

\section{Sources:}

(1) FY 2002 Budget Request - Data Bucket Report for Analysis Tools and Design Strategies Program (internal BTS document).

(2) Interview with Dru Crawley, Program Manager, August 22, 2001. 


\section{Lighting and Appliance Standards}




\section{Lighting and Appliance Standards}

\section{Building Equipment and Materials Decision Unit}

\section{Program Objective: ${ }^{(1)}$}

The Lighting and Appliance Standards program achieves significant energy savings, consumer cost savings, and reduced air emissions through standards rulemaking. The program also prescribes test procedures that measure energy efficiency and energy use and that estimate the annual operating cost of each appliance.

\section{Long-Term Goal:(1)}

The program's long-term goal is to set efficiency standards that lead to substantial increases in the average efficiency of new building equipment.

\section{Market Segment:}

\section{Target Market}

- Market Description: All residential and commercial equipment covered by the appropriate legislation. $(2,3)$

- Size of Market: All residential and commercial equipment in the market.

\section{Methodology}

For FY 2003, the energy savings from equipment standards activities were primarily based on a PNNLdeveloped spreadsheet to support an EPAct screening analysis conducted in late 1999 and early 2000. This spreadsheet was used to estimate the energy savings from various levels of standards for nearly 40 types of equipment covered by EPAct. The spreadsheet results were used to identify products that could achieve significant energy savings beyond the efficiency levels set in the recent ASHRAE 90.1-1999 publication. ${ }^{(4)}$

For FY 2003, the EPAct standards were assumed to continue with the products having the potential for additional energy savings. These products include boilers, three-phase residential-size cooling equipment, packaged terminal air conditioning, packaged terminal heat pump equipment, and large rooftop airconditioning equipment. Energy-savings' estimates for these products based on the spreadsheet are shown in the next section.

The FY 2003 savings for this program also included an estimate for revised standards for residential gas

\section{Lighting and Appliance Standards}

\section{Program Type:}

Codes and standards for equip-

ment efficiency

Target Market:

All sectors in all climate zones

End Uses:

All end uses and all fuel types

Unit of Measurement:

Efficiency of specific equipment

Modeling Tool:

NEMS

Spreadsheet

Program Manager:

Carl Adams

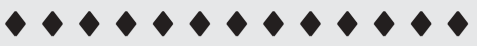

FY 2003 Benefits

Primary Energy Savings (TBtu)

\begin{tabular}{llll}
2003 & 2005 & 2010 & 2020 \\
\hline 0 & 7.8 & 106.1 & 387.1
\end{tabular}

Carbon Equivalent Reductions (MMTCE)

\begin{tabular}{llll}
2003 & 2005 & 2010 & 2020 \\
\hline .000 & .145 & 2.128 & 7.38
\end{tabular}

Consumer Cost Savings (million \$)

\begin{tabular}{llll}
2003 & 2005 & 2010 & 2020 \\
\hline 0 & 49 & 705 & 2969
\end{tabular}

Building Equipment and Materials - 27 


\section{Lighting and Appliance Standards}

\section{Building Equipment and Materials Decision Unit}

furnaces. This program was modeled in the NEMS residential model. In the baseline version of the model used by EIA for its Annual Energy Outlook 2000,(5) a wide range of the furnace efficiencies was available: $78 \%, 80 \%, 84 \%, 88 \%$, and $96 \%$. To estimate the standards program for furnaces, the lowest two efficiency levels were assumed to not meet the revised standard after 2007. In addition, savings related to standards covering distribution transformers were calculated in a spreadsheet based on a 1992 study conducted by Geller and Nadel.(6)

\section{Commercial Products:}

Based on the spreadsheet EPACT_SA.XLS (essentially identical to the spreadsheet installed on the BTS website for public comment subsequent to the EPAct screening analysis), Tables 1 and 2 summarize the efficiency assumptions and energy savings results for products that will undergo further analysis by DOE/BTS. The key assumptions and results were summarized for 12 cooling products in Table 1 and for boilers and a high-capacity instantaneous water heater in Table 2. Cumulative savings, shown in the last column in both tables, were based on the savings from the effective date of the standards through 2030.

\section{Distribution Transformers:}

Distribution transformers convert highvoltage electricity from distribution centers to lower-voltage electricity for use at the household level. During this conversion process, a small fraction of heat is lost. Rules are being written to reduce the amount of heat loss during this conversion process.

Savings' estimates for a distribution transformer standard were based on a study conducted by Geller and Nadel.(5) The study assumed the following:

- Savings of 80 watts per unit

- Annual sales of 1.2 million units

- $20 \%$ sales complying with the new level without the standard

- 8,760 annual operating hours per unit

- 13-year life of equipment.

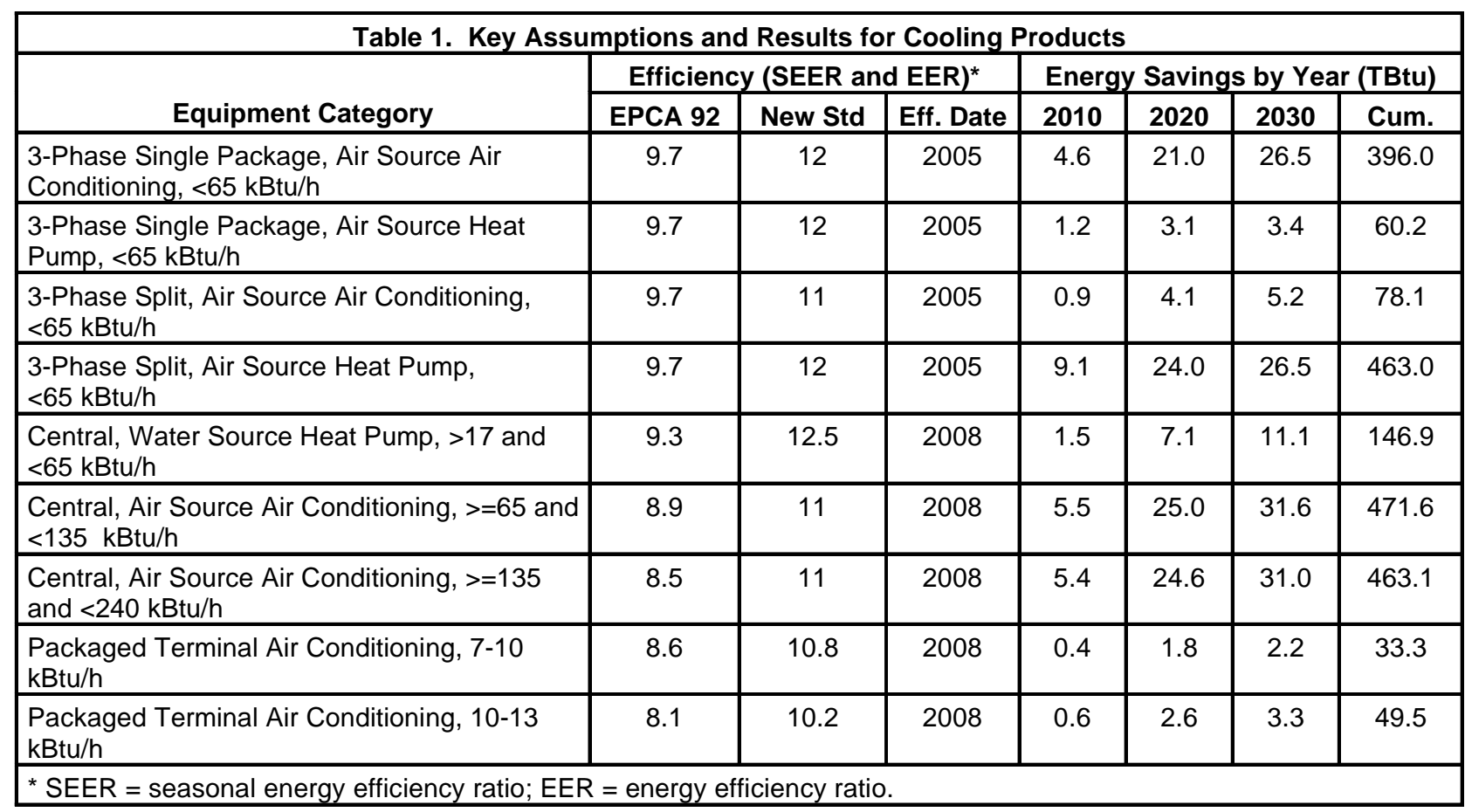




\title{
Lighting and Appliance Standards
}

\author{
Building Equipment and Materials Decision Unit
}

Table 2. Key Assumptions and Results for Boilers and a High-Capacity Instantaneous Water Heater

\begin{tabular}{|l|l|l|l|l|r|r|r|}
\hline & \multicolumn{3}{c|}{ Efficiency (SEER and EER) } & \multicolumn{3}{|c|}{ Energy Savings by Year (TBtu) } \\
\cline { 2 - 7 } \multicolumn{1}{c|}{ Equipment Category } & EPCA 92 & New Std & Eff. Date & $\mathbf{2 0 1 0}$ & $\mathbf{2 0 2 0}$ & $\mathbf{2 0 3 0}$ & Cum. \\
\hline Pkg'd Boilers, Gas, 400 kBtu/h, Hot Water & $75 \%$ & $78 \%$ & 2008 & 0.2 & 0.9 & 1.7 & 19.7 \\
\hline Pkg'd Boilers, Gas, 800 kBtu/h, Hot Water & $75 \%$ & $78 \%$ & 2008 & 0.4 & 2.0 & 3.7 & 43.0 \\
\hline Pkg'd Boilers, Gas, 1500 kBtu/h, Hot Water & $75 \%$ & $78 \%$ & 2008 & 0.1 & 0.7 & 1.2 & 14.2 \\
\hline Pkg'd Boilers, Gas, 3000 kBtu/h, HW & $75 \%$ & $80 \%$ & 2008 & 0.2 & 0.7 & 1.3 & 15.2 \\
\hline Pkg'd Boilers, Gas, 400 kBtu/h, Steam & $72 \%$ & $76 \%$ & 2008 & 0.1 & 0.6 & 1.1 & 12.6 \\
\hline Pkg'd Boilers, Gas, 800 kBtu/h, Steam & $72 \%$ & $76 \%$ & 2008 & 0.4 & 1.6 & 3.0 & 34.5 \\
\hline Pkg'd Boilers, Gas, 1500 kBtu/h, Steam & $72 \%$ & $79 \%$ & 2008 & 0.3 & 1.2 & 2.3 & 26.7 \\
\hline Pkg'd Boilers, Gas, 3000 kBtu/h, Steam & $72 \%$ & $80 \%$ & 2008 & 0.2 & 0.9 & 1.7 & 19.2 \\
\hline Instantaneous Water Heaters, 1000 kBtu/h & $80 \%$ & $83 \%$ & 2008 & 1.0 & 4.4 & 5.6 & 83.3 \\
\hline
\end{tabular}

The savings estimate of 80 watts per unit installed was multiplied by the estimated hours of operation and then multiplied by the forecasted number of units installed.

\section{Residential Gas Furnaces:}

Rules related to the efficiency of residential gas furnaces are being written with the anticipated adoption date of 2008. Savings for residential gas furnaces were estimated using NEMS and the following assumptions:

- Proposed residential gas furnace efficiency of $82 \%$ annual fuel utilization efficiency

- Introduction date of 2008

- Cost of $\$ 1400$.

\section{Non-Energy Benefits:(1)}

Reduced $\mathrm{CO}_{2}$ and $\mathrm{SO}_{\mathrm{x}}$ emissions, reduced water consumption from plumbing equipment, increased life of equipment operating at cooler temperatures, and reduced first costs that transform new technologies into commodities.

\section{Program Strategy (\% of budget): $:^{(1)}$}

- Research and Development - 0\%

- Market Transformation - 0\%

- Codes and Standards - $100 \%$.

\section{Sources:}

(1) FY 2002 Budget Request - Data Bucket Report for the Lighting and Appliance Standards Program (internal BTS document).

(2) National Appliance Energy Conservation Act of 1987, Public Law 100-12.

(3) Energy Policy Act of 1992, Public Law 102-486.

(4) ASHRAE 90.1-1999, “Energy Standard for Buildings Except Low-Rise Residential Buildings," American Society of Heating, Refrigeration, and Air-Conditioning Engineers.

(5) Annual Energy Outlook 2000. 1999. Energy Information Administration, Washington, D.C.

(6) Geller, H. and S. Nadel. 1992. "Consensus National Efficiency Standards for Lamps, Motors, Showerheads and Faucets, and Commercial HVAC Equipment." In American Council for an Energy Efficient Economy Proceedings, pp. 6.71-6.82. 


\section{Appendix A Baseline Assumptions}




\section{Appendix A \\ Baseline Scenario and Inputs for FY 2003 GPRA Metrics}

To obtain the GPRA metrics for FY 2003, the following baseline scenario and inputs were used. This information is common to all programs analyzed within BESET.

\section{Building Stock}

Building stock estimates are used to estimate the total energy savings at the national level for each program. Residential and commercial new and existing building stock totals for all years through 2020 were provided by EIS's Annual Energy Outlook 2001. The stock estimates have been developed for each market segment (e.g., building type, building vintage, and region) based on the following assumptions.

- Residential Single-Family and Multifamily Housing

- $60 \%$ of the existing building stock is in the north.

- $\quad 40 \%$ of the existing building stock is in the south.

- New stock is divided evenly across regions.

- Residential Manufactured Housing

- $\quad 48 \%$ of the existing building stock is in the north.

- $52 \%$ of the existing building stock is in the south.

- $\quad 45 \%$ of the new building stock is in the north.

- $55 \%$ of the new building stock is in the south.

- Commercial Buildings

- $59 \%$ of the existing building stock is in the north.

- $41 \%$ of the existing building stock is in the south.

- $55 \%$ of the new building stock is in the north.

- $\quad 45 \%$ of the new building stock is in the south.

By using the assumptions listed above, the building stock numbers are segmented by building vintage and region. The base year is 2003, and all construction beginning with 2003 is considered "new."

The building stock was disaggregated into "north" and "south" regions by using the EIA climate zones published in the Residential Energy Consumption Survey and the Commercial Buildings Energy Consumption Survey. Climate zones 1 through 3 (i.e., zones with $>4,000$ heating-degree days) were designated as the "north" region, and zones 4 and 5 (i.e., zones with $<4,000$ heatingdegree days) were defined as the "south" regions (see Figure 1). Using this method, approximate percentages of north and south existing units and new construction were estimated.

\section{Existing Equipment Market Shares}

Equipment market shares were broken out by market segment and are estimated from Residential Energy Consumption Survey, the Commercial Buildings Energy Consumption Survey, and original PNNL efforts by Dave Belzer. 


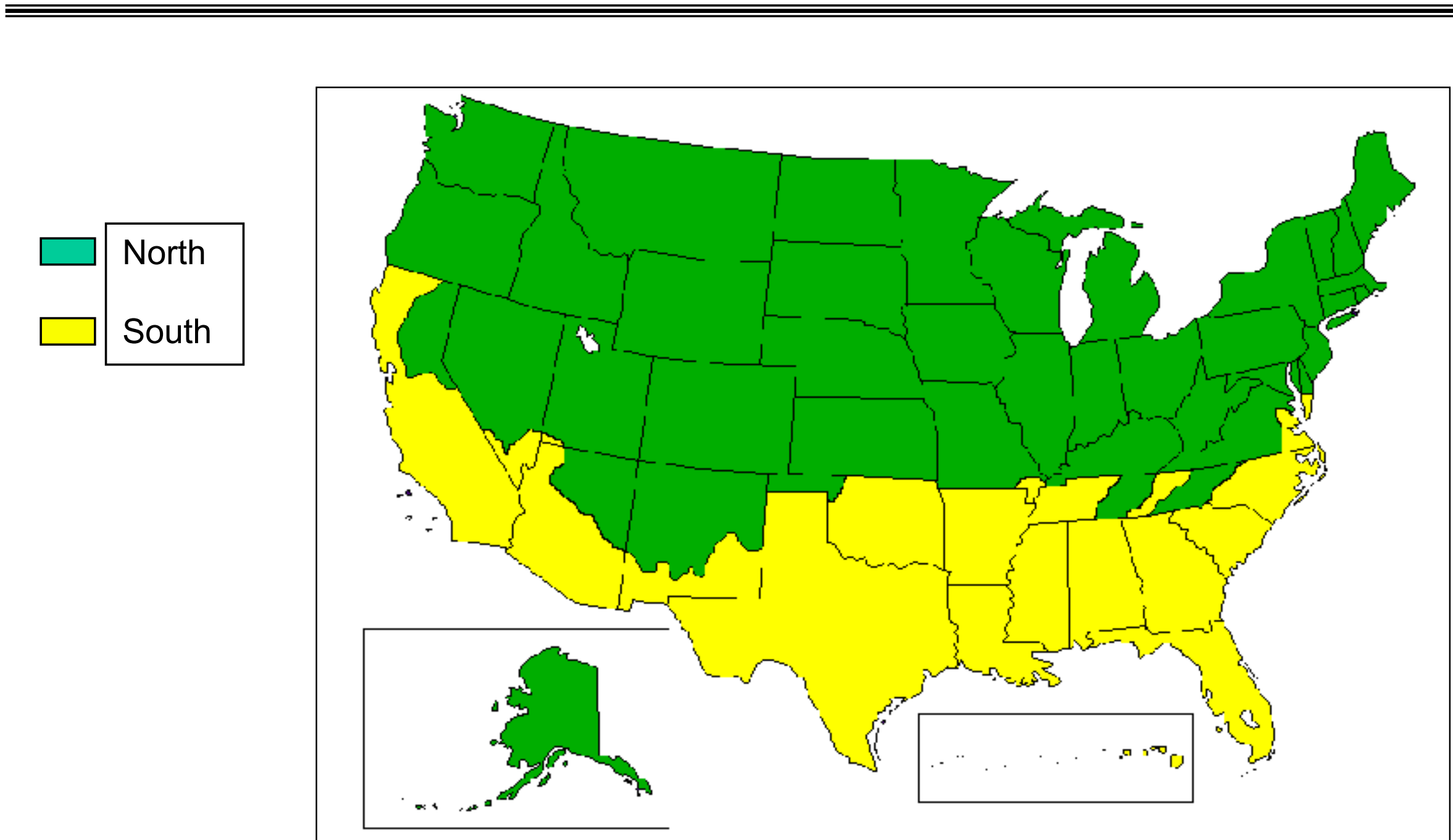

Figure 4. Regions for BTS GPRA Metrics 


\section{Existing Equipment Efficiencies}

The efficiency of equipment stock was developed from EIA's 1995 Annual Energy Outlook and input from Program Managers. Where applicable, the assumed stock efficiency increased to meet equipment standards.

\section{End-Use Loads}

End-use loads represent the baseline energy use per square foot (commercial) or per unit (residential) for heating, cooling, water heating, and lighting uses. Previous to the FY2002 effort, baseline end-use loads were averaged over all building types and distinguished only by building sector (commercial or residential), building vintage (new or existing), and climate zone (north or south). To more accurately reflect the savings of programs targeting specific buildings, baseline end-use loads are now distinguished by building types (e.g. assembly, education, multi-family, etc) as well as by vintage and climate zone. End-use loads were updated in June 2000 with energy use information derived from the Facility Energy Decision System (FEDS) to reflect current energy technology and consumption behavior.

\section{Equipment Life}

Equipment replacement factors are used in calculating the number of units representing the potential target market for programs targeting specific pieces of equipment. BTS technologies are assumed to compete only with "new" units. For new buildings, all units are considered new; therefore, the replacement factor for equipment in new buildings is 1.0.

For existing buildings, only units scheduled to be replaced are considered to be eligible for the BTS technology. In other words, the BTS technology is assumed not to replace a piece of equipment unless it was going to be replaced anyway. The replacement factor for existing buildings for a given year is based on the percentage of the equipment that is expected to turn over in that period (e.g., the factor for 2005 represents the percentage turnover from 2003 to 2005; the factor for 20010 represents the turnover percentage from 2005 to 2010, etc.). The replacement factors are based on the specific equipment's life expectancy. Baseline factors are taken from Appliance Magazine, NEMS, and the BTS Core Data Book.

\section{Sources}

Annual Energy Outlook, 1995, Energy Information Administration, Washington, D.C.

Annual Energy Outlook, 2001, Energy Information Administration, Washington, D.C.

BTS Core Databook, internal BTS document, U.S. Department of Energy.

Appliance Magazine. 1998. "Life Expectancy/Replacement Picture," 55(9):71.

Facility Energy Decision System (FEDS) 2000. Federal Energy Management Program (FEMP), Department of Energy, Washington, D.C. 


\section{Appendix B GPRA Data Call}




\section{APPENDIX B}

\section{Calculation Methodologies}

This appendix provides methodologies for calculating the metrics requested in the GPRA2003 Data Call. The general framework for the methodology is depicted in the Figure B-1 below. Additional details are provided in the sections that follow.

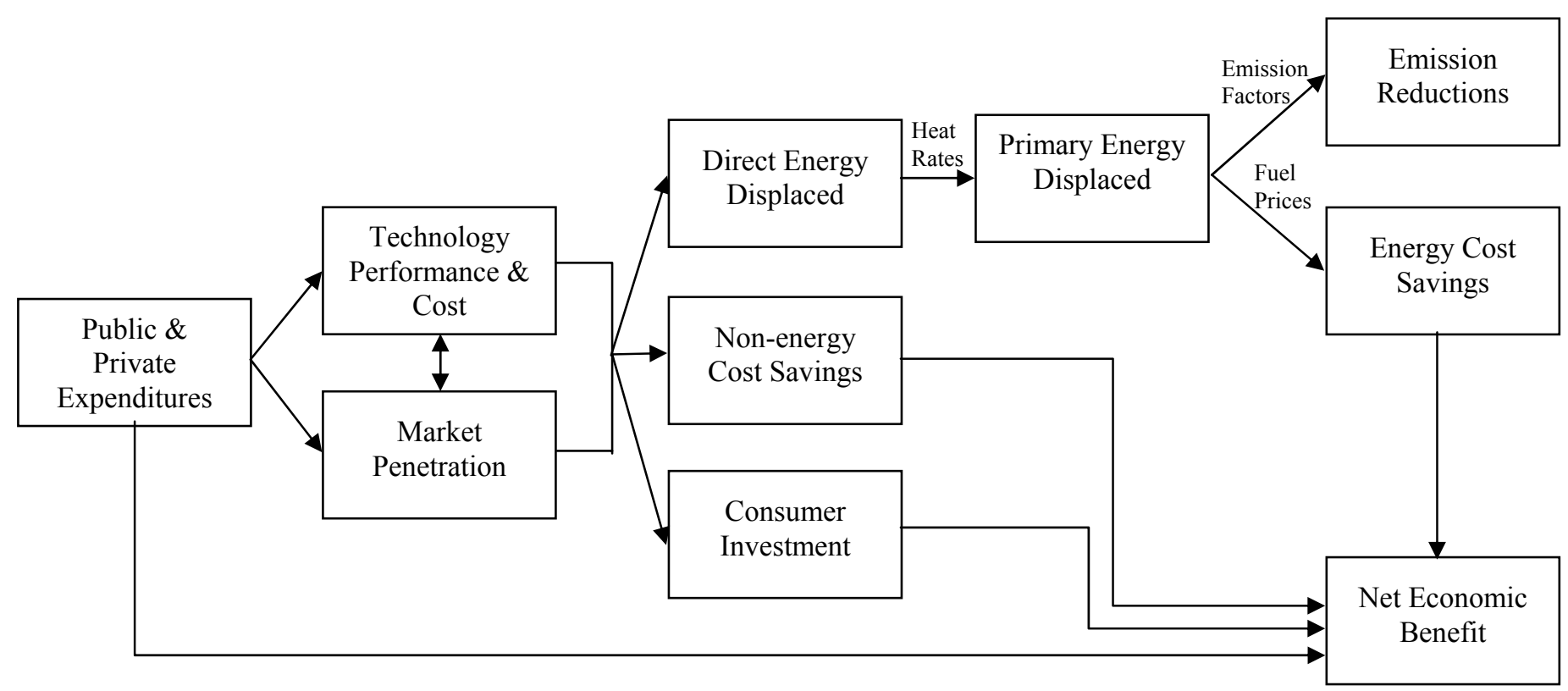

Figure B-1: EERE GPRA2003 Calculation methodology framework 


\section{Calculating Public \& Private Expenditures}

Programs should identify annual and cumulative expenditures by EERE, other government agencies, and the private sector.

\section{Calculating Public \& Private Expenditures - An Example}

An EERE program is funding R\&D for an advanced technology that will reduce natural gas and electricity consumption in an industrial process. The EERE program has provided $\$ 5$ million in funding through 2002 and plans to provide additional funding from 2003 through 2007 . No other government funding is being provided, but the private sector is matching EERE's funding (and continues through 2009). The first step is to identify the stream of historical and projected annual expenditures.

\section{Annual Expenditures (Millions nominal \$)}

\begin{tabular}{|l|c|c|c|c|c|c|c|c|c|c|}
\cline { 2 - 10 } Step (1) & 1998 & 1999 & 2000 & 2001 & 2002 & 2003 & 2004 & 2005 & 2006 & 2007 \\
\hline EERE & 1.000 & 1.000 & 1.000 & 1.000 & 1.000 & 1.000 & 2.000 & 2.000 & 1.000 & 1.000 \\
\hline Other Government & 0.000 & 0.000 & 0.000 & 0.000 & 0.000 & 0.000 & 0.000 & 0.000 & 0.000 & 0.000 \\
\hline Private Sector & 1.000 & 1.000 & 1.000 & 1.000 & 1.000 & 1.000 & 2.000 & 2.000 & 1.000 & 1.000 \\
\hline
\end{tabular}

The second step is to apply the GDP implicit price deflators from Appendix C of the data call.

\section{GDP Implicit Price Deflators}

$(1999=1.000)$

\begin{tabular}{|l|c|c|c|c|c|c|c|c|c|c|}
\cline { 2 - 11 } Step (2) & 1998 & 1999 & 2000 & 2001 & 2002 & 2003 & 2004 & 2005 & 2006 & 2007 \\
\hline Price Deflator & 0.985 & 1.000 & 1.022 & 1.044 & 1.064 & 1.087 & 1.111 & 1.135 & 1.158 & 1.180 \\
\hline
\end{tabular}

This results in annual expenditures in 1999 dollars. Expenditures before 2003 will be included in cumulative expenditures (see below). Expenditures from 2003-2007 will be reported under annual expenditures. These become the basis of revision after the budget is received.

\section{Annual Expenditures \\ (Millions 1999 \$)}

\begin{tabular}{|l|c|c|c|c|c|c|c|c|c|c|}
\cline { 2 - 10 } Step (3) & 1998 & 1999 & 2000 & 2001 & 2002 & 2003 & 2004 & 2005 & 2006 & 2007 \\
\hline EERE & 1.015 & 1.000 & 0.978 & 0.958 & 0.940 & 0.920 & 1.800 & 1.762 & 0.863 & 0.847 \\
\hline Other Government & 0.000 & 0.000 & 0.000 & 0.000 & 0.000 & 0.000 & 0.000 & 0.000 & 0.000 & 0.000 \\
\hline Private Sector & 1.015 & 1.000 & 0.978 & 0.958 & 0.940 & 0.920 & 1.800 & 1.762 & 0.863 & 0.847 \\
\hline
\end{tabular}

The fourth step is to identify cumulative expenditures. This is done by adding annual expenditures to cumulative expenditures for the previous year. 


\section{Cumulative Expenditures \\ (Millions 1999 \$)}

\begin{tabular}{|l|c|c|c|c|c|c|c|c|c|c|}
\cline { 2 - 9 } Step (4) & 2003 & 2004 & 2005 & 2006 & 2007 & 2010 & 2015 & 2020 & 2025 & 2030 \\
\hline EERE & 5.810 & 7.610 & 9.372 & 10.235 & 11.082 & 11.082 & 11.082 & 11.082 & 11.082 & 11.082 \\
\hline Other Government & 0.000 & 0.000 & 0.000 & 0.000 & 0.000 & 0.000 & 0.000 & 0.000 & 0.000 & 0.000 \\
\hline Private Sector & 5.810 & 7.610 & 9.372 & 10.235 & 11.082 & 12.730 & 12.730 & 12.730 & 12.730 & 12.730 \\
\hline
\end{tabular}




\section{Identifying Technology Performance and Cost}

Technology performance and cost information should be provided for the advanced EERE technology and the baseline technology. The baseline technology should represent the next best alternative to the advanced EERE technology. It should not represent the average technology in the market or the technology being replaced. As a general rule, programs should use the technology characteristics contained in EIA's Annual Energy Outlook 2001 (AEO2001) as the baseline. In instances where the AEO2001 does not contain baseline information, programs should draw such information from other credible sources.

\section{Identifying Technology Performance and Cost - An Example}

An EERE program is funding R\&D for an advanced technology that will reduce natural gas and electricity consumption in an industrial process. The EERE-funded R\&D is expected to accelerate the market introduction of the technology from 2014 to 2009.

Step 1 consists of identifying the relevant performance parameters for 1) the advanced technology with EERE involvement, 2) the advanced technology without EERE involvement, and 3) the next best alternative. The annual energy consumption of the process using the next best alternative technology is 400 million $\mathrm{ft}^{3}$ of natural gas and 20 million $\mathrm{kWh}$ of electricity in 2000. These levels are expected to decline by $0.20 \%$ per year. The annual energy consumption with the

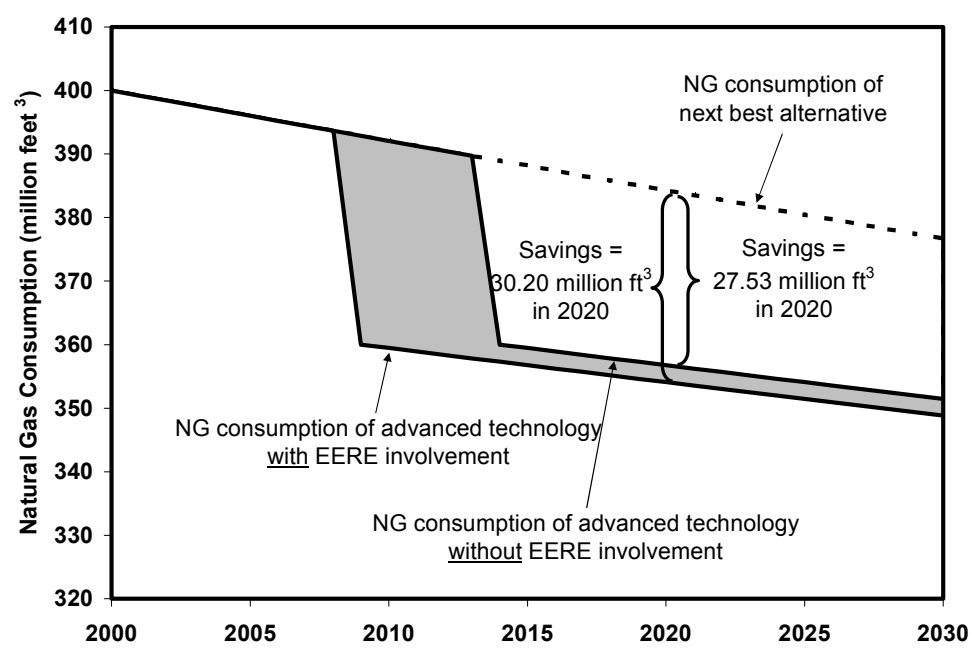

Figure B-2: Unit energy consumption of advanced EERE technology and next best alternative advanced technology is expected to be 360 million $\mathrm{ft}^{3}$ of natural gas and 18 million $\mathrm{kWh}$ of electricity per year, levels estimated to improve by $0.15 \%$ per year through 2030 (see Figure B-2 and the table below).

\section{Unit Energy Consumption}

\begin{tabular}{|c|c|c|c|c|c|c|c|c|c|c|}
\hline Step (1) & 2003 & 2004 & 2005 & 2006 & 2007 & 2010 & 2015 & 2020 & 2025 & 2030 \\
\hline \multicolumn{11}{|l|}{ Natural Gas (million $\mathrm{ft}^{3}$ ) } \\
\hline Adv. Tech. With EERE & --- & --- & --- & --- & --- & 359.46 & 356.77 & 354.10 & 351.46 & 348.83 \\
\hline Adv. Tech. Without EERE & --- & --- & --- & --- & --- & --- & 359.46 & 356.77 & 354.10 & 351.46 \\
\hline Next Best Alternative & 397.60 & 396.81 & 396.02 & 395.22 & 394.43 & 392.07 & 388.17 & 384.30 & 380.47 & 376.68 \\
\hline \multicolumn{11}{|l|}{ Electricity (million kWh) } \\
\hline Adv. Tech With EERE & --- & --- & --- & $\overline{---}$ & --- & 17.97 & 17.84 & 17.71 & 17.57 & 17.44 \\
\hline Adv. Tech. Without EERE & --- & --- & -- & 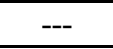 & -- & --- & 17.97 & 17.84 & 17.71 & 17.57 \\
\hline Next Best Alternative & 19.88 & 19.84 & 19.80 & 19.76 & 19.72 & 19.60 & 19.41 & 19.22 & 19.02 & 18.83 \\
\hline
\end{tabular}


In step 2 the unit energy savings of the advanced technology are calculated relative to the next best alternative. The energy savings of the advanced technology with EERE involvement is about 30.20 million $\mathrm{ft}^{3}$ of natural gas and 1.51 million $\mathrm{kWh}$ of electricity per year in 2020 . The energy savings of the advanced technology without EERE involvement is about 27.53 million $\mathrm{ft}^{3}$ of natural gas and 1.38 million $\mathrm{kWh}$ of electricity per year in 2020 .

\section{Unit Energy Savings}

\begin{tabular}{|c|c|c|c|c|c|c|c|c|c|c|}
\hline Step (2) & 2003 & 2004 & 2005 & 2006 & 2007 & 2010 & 2015 & 2020 & 2025 & 2030 \\
\hline \multicolumn{11}{|l|}{ Natural Gas (million $\mathrm{ft}^{3}$ ) } \\
\hline Adv. Tech With EERE & --- & --- & --- & --- & --- & 32.61 & 31.39 & 30.20 & 29.02 & 27.85 \\
\hline Adv. Tech Without EERE & --- & --- & --- & --- & --- & --- & 28.71 & 27.53 & 26.37 & 25.23 \\
\hline \multicolumn{11}{|l|}{ Electricity (million kWh) } \\
\hline Adv. Tech With EERE & --- & --- & --- & --- & --- & 1.63 & 1.57 & 1.51 & 1.45 & 1.39 \\
\hline Adv. Tech Without EERE & --- & --- & --- & --- & --- & --- & 1.44 & 1.38 & 1.32 & 1.26 \\
\hline
\end{tabular}

Step 3 identifies the capital costs for each technology. These are shown in the table below and in Figure B-3.

\section{Capital Cost (Millions 1999 \$)}

\begin{tabular}{|c|c|c|c|c|c|c|c|c|c|c|}
\hline Step (3) & 2003 & 2004 & 2005 & 2006 & 2007 & 2010 & 2015 & 2020 & 2025 & 2030 \\
\hline Adv. Tech. With EERE & --- & --- & --- & --- & --- & 6.50 & 6.03 & 5.59 & 5.18 & 4.81 \\
\hline Adv. Tech. Without EERE & --- & --- & --- & --- & --- & --- & 6.50 & 6.03 & 5.59 & 5.18 \\
\hline Next Best Alternative & 5.82 & 5.76 & 5.71 & 5.65 & 5.59 & 5.43 & 5.16 & 4.91 & 4.67 & 4.44 \\
\hline
\end{tabular}

In step 4 the incremental capital costs of the advanced technology are calculated relative to the next best alternative.

\section{Incremental Capital Cost (Millions 1999 \$)}

\begin{tabular}{|c|c|c|c|c|c|c|c|c|c|c|}
\hline Step (4) & 2003 & 2004 & 2005 & 2006 & 2007 & 2010 & 2015 & 2020 & 2025 & 2030 \\
\hline Adv. Tech. With EERE & --- & --- & --- & --- & --- & 1.07 & 0.87 & 0.68 & 0.52 & 0.37 \\
\hline Adv. Tech. Without EERE & --- & --- & --- & --- & --- & --- & 1.34 & 1.12 & 0.92 & 0.74 \\
\hline
\end{tabular}




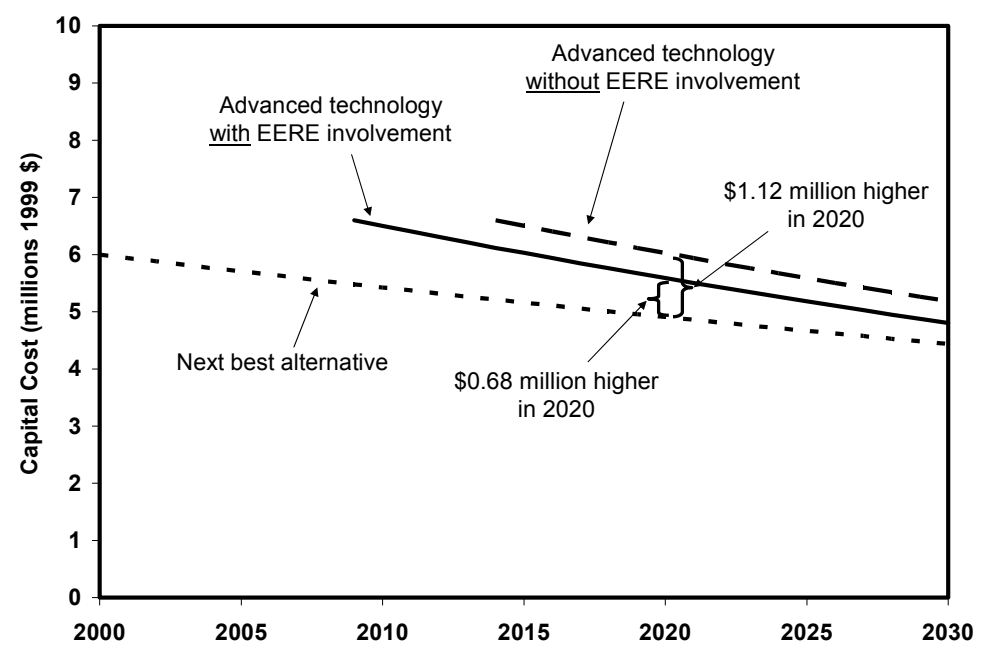

Figure B-3: Capital costs of advanced EERE technology and next best alternative

Step 5 calculates annualized capital cost by dividing capital cost by the lifetime of the technology.

\section{Annualized Capital Cost \\ (Millions 1999 \$/year)}

\begin{tabular}{|c|c|c|c|c|c|c|c|c|c|c|}
\hline \multirow[b]{2}{*}{ Step (5) } & & & & & & & & & & \\
\hline & 2003 & 2004 & 2005 & 2006 & 2007 & 2010 & 2015 & 2020 & 2025 & 2030 \\
\hline Adv. Tech. With EERE & --- & --- & --- & --- & --- & 0.65 & 0.60 & 0.56 & 0.52 & 0.48 \\
\hline Adv. Tech. Without EERE & --- & --- & --- & --- & --- & --- & 0.65 & 0.60 & 0.56 & 0.52 \\
\hline Next Best Alternative & 0.58 & 0.58 & 0.57 & 0.57 & 0.56 & 0.54 & 0.52 & 0.49 & 0.47 & 0.44 \\
\hline
\end{tabular}

Step 6 calculates the incremental annualized capital costs of the advanced technology relative to the next best alternative.

\section{Incremental Annualized Capital Cost (Millions 1999 \$/year)}

\begin{tabular}{|c|c|c|c|c|c|c|c|c|c|c|}
\hline Step (6) & 2003 & 2004 & 2005 & 2006 & 2007 & 2010 & 2015 & 2020 & 2025 & 2030 \\
\hline Adv. Tech. With EERE & --- & --- & --- & --- & --- & 0.11 & 0.09 & 0.07 & 0.05 & 0.04 \\
\hline Adv. Tech. Without EERE & --- & --- & --- & --- & --- & --- & 0.13 & 0.11 & 0.09 & 0.07 \\
\hline
\end{tabular}


Step 7 identifies the O\&M costs for each technology.

O\&M Costs

(Millions 1999 \$)

\begin{tabular}{|l|c|c|c|c|c|c|c|c|c|c|}
\cline { 2 - 9 } \multicolumn{1}{l|}{ Step (7) } & 2003 & 2004 & 2005 & 2006 & 2007 & 2010 & 2015 & 2020 & 2025 & 2030 \\
\hline Adv. Tech. With EERE & --- & --- & --- & --- & --- & 0.0650 & 0.0603 & 0.0559 & 0.0518 & 0.0481 \\
\hline Adv. Tech. Without EERE & --- & --- & --- & --- & --- & --- & 0.0650 & 0.0603 & 0.0559 & 0.0518 \\
\hline Next Best Alternative & 0.0582 & 0.0576 & 0.0571 & 0.0565 & 0.0559 & 0.0543 & 0.0516 & 0.0491 & 0.0467 & 0.0444 \\
\hline
\end{tabular}

Step 8 calculates the incremental O\&M costs of the advanced technology relative to the next best alternative.

\section{Incremental O\&M Costs (Millions 1999 \$)}

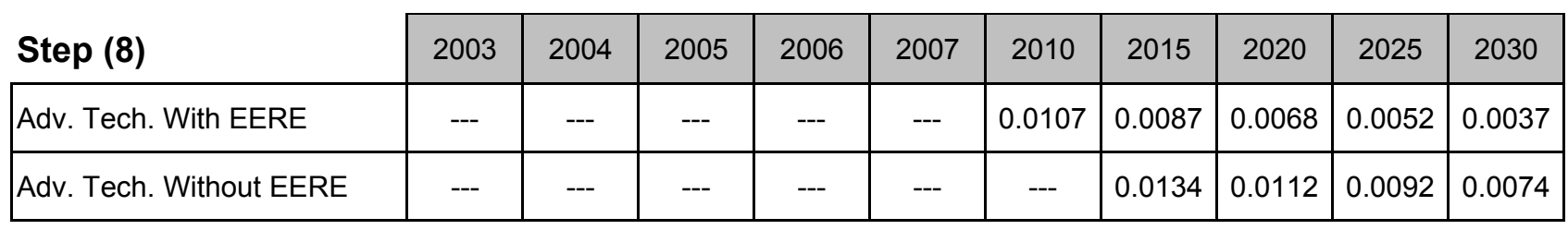




\section{Identifying Market Penetration Information}

Market penetration levels should be identified for the advanced EERE technology and the baseline technology. As a general rule, programs should use the market penetration levels contained in EIA's Annual Energy Outlook 2001 (AEO2001) as the baseline. In instances where the AEO2001 does not contain baseline information, programs should draw such information from other credible sources.

The market penetration information for the advanced EERE technology should include a commercialization year and major milestones leading to commercialization. In identifying a commercialization year, programs should consider that it usually takes several years to move from research and development to the introduction of a product into the marketplace. The timeframe in Figure B-4 (ADL, 2000) should serve as a guide.

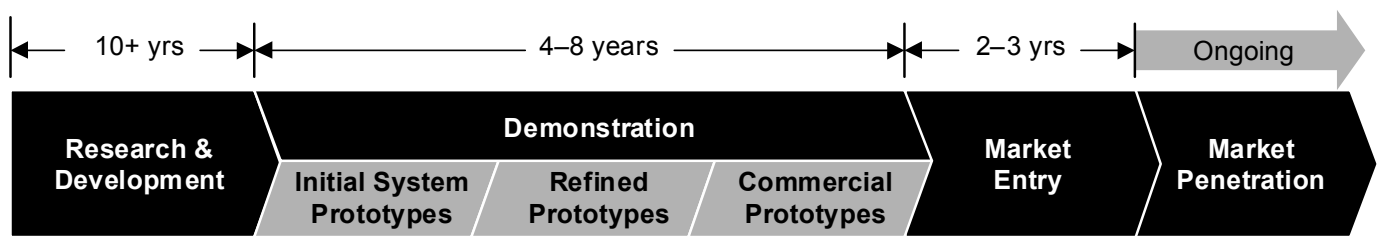

Figure B-4. The Technology Development Process

\section{Identifying Market Penetration - An Example}

Step 1 identifies the commercialization year and the major milestones leading to commercialization. In this example, major milestones include an initial prototype in 2002, a refined prototype in 2004, and a commercial prototype in 2007, with commercialization in 2009.

\begin{tabular}{|c|c|c|c|c|c|c|c|c|c|c|}
\hline Step (1) & 2002 & 2003 & 2004 & 2005 & 2006 & 2007 & 2008 & 2009 & 2010 & 2011 \\
\hline Initial Prototype & \multicolumn{10}{|l|}{$x$} \\
\hline Refined Prototype & \multicolumn{10}{|c|}{$x$} \\
\hline Commercial Prototype & \multicolumn{10}{|c|}{$\mathrm{x}$} \\
\hline Commercialization & & & & & & \multicolumn{5}{|c|}{$x$} \\
\hline
\end{tabular}

Step 2 identifies the market penetration levels of the advanced technology with and without EERE involvement resulting in the net penetration of the advanced technology. In this example, the market penetration curve of the advanced technology with EERE involvement is the same as market penetration curve without EERE involvement, except that it is shifted to the left by five 
years. ${ }^{1}$ The net market penetration is the difference between the two curves, or the shaded area in Figure B-5. The total market size in both cases is 500 units.

With EERE involvement, 32 units were added in 2020, for a cumulative total of 106 . Without EERE involvement, 5 units would have been added in 2020, for a cumulative total of 14 . Thus, the net number of installations added in 2020 was 27 for a net cumulative total of 92 units. Cumulative and annual market penetration levels are shown in the tables below.

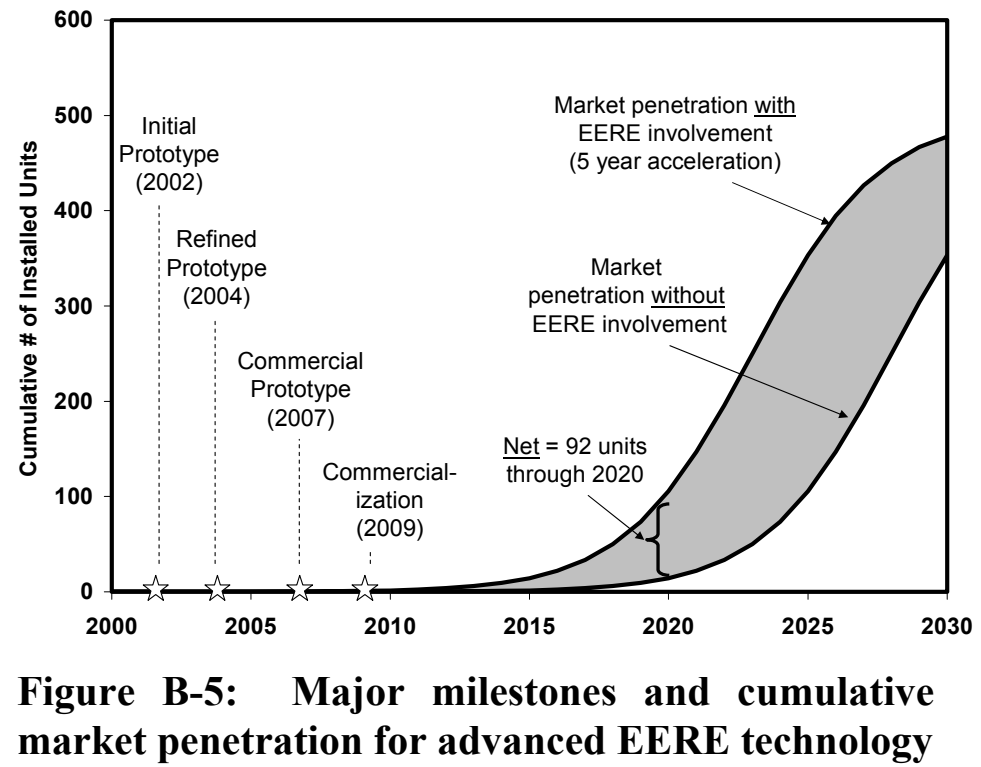

\section{Market Penetration}

(\# units)

\begin{tabular}{|c|c|c|c|c|c|c|c|c|c|c|}
\hline Step (2) & 2003 & 2004 & 2005 & 2006 & 2007 & 2010 & 2015 & 2020 & 2025 & 2030 \\
\hline \multicolumn{11}{|l|}{ Cumulative Penetration } \\
\hline Adv. Tech. With EERE & --- & --- & --- & --- & --- & 2 & 14 & 106 & 353 & 478 \\
\hline Adv. Tech. Without EERE & --- & --- & --- & --- & --- & --- & 2 & 14 & 106 & 353 \\
\hline \multicolumn{11}{|l|}{ Annual Penetration } \\
\hline Adv. Tech. With EERE & --- & --- & --- & --- & --- & 1 & 5 & 32 & 49 & 11 \\
\hline Adv. Tech. Without EERE & --- & --- & --- & --- & -- & --- & 1 & 5 & 32 & 49 \\
\hline
\end{tabular}

\footnotetext{
${ }^{1}$ The market penetration curve, measured in percentage of market captured, is shifted to the left by five years. In this example, zero market growth is assumed over the market penetration period. Thus, the market penetration curve, measured in absolute numbers (\# units), is the same. If market growth or decline is projected, then the two curves, measured in absolute numbers, will differ.
} 


\section{Calculating Direct Energy Displaced}

The amount of direct energy displaced is a function of per unit energy displacement and the number of units in the market. Direct energy displaced may be measured in a number of ways. The incremental direct energy displaced is the amount of energy displaced for the number of units installed in a year. The annual direct energy displaced represents the amount of energy displaced in a year for the units installed thus far. It is the summation of incremental energy benefits over the years of interest

\section{Calculating Direct Energy Displaced - An Example}

This example continues from above. In Step 1 the amount of incremental direct energy displaced is calculated. The incremental energy benefit of the 32 units installed in 2020 is a combination of three calculations. First, there are 966 million $\mathrm{ft}^{3}$ of natural gas savings from the advanced technology with EERE involvement ( 32 units $x 30.20$ million $\mathrm{ft}^{3} /$ unit). Second, these savings are reduced by the 138 million $\mathrm{ft}^{3}$ of natural gas for units that would have been installed without EERE involvement ( 5 units $x 27.53$ million $\mathrm{ft}^{3} / \mathrm{unit}$ ). Third, there are further reductions of 1 million $\mathrm{ft}^{3}$ in natural gas due to increased consumption of stock turnover (the per unit savings is less than it was ten years earlier - the lifetime of the advanced technology). Thus, the amount of incremental energy displaced for the 32 installed in 2020 would be about 827 million $\mathrm{ft}^{3}$ of natural gas. Similar calculations for electricity show 0.04 billion $\mathrm{kWh}$ of electricity displaced.

\section{Incremental Direct Energy Displaced}

\begin{tabular}{|l|c|c|c|c|c|c|c|c|c|c|}
\cline { 2 - 10 } Step (1) & 2003 & 2004 & 2005 & 2006 & 2007 & 2010 & 2015 & 2020 & 2025 & 2030 \\
\hline $\begin{array}{l}\text { Direct Electricity Displaced } \\
\text { (billion kWhs) }\end{array}$ & 0.00 & 0.00 & 0.00 & 0.00 & 0.00 & 0.00 & 0.01 & 0.04 & 0.03 & -0.05 \\
\hline $\begin{array}{l}\text { Direct Natural Gas Displaced } \\
\text { (billion cubic feet) }\end{array}$ & 0.00 & 0.00 & 0.00 & 0.00 & 0.00 & 0.02 & 0.14 & 0.83 & 0.57 & -0.99 \\
\hline
\end{tabular}

Step 2 calculates the amount of annual direct energy displaced. When the incremental benefits are summed for all 106 units penetrating the market through 2020 and replacements accounted for, the annual direct energy displaced in 2020 is about 2.8 billion $\mathrm{ft}^{3}$ of natural gas and 0.14 billion $\mathrm{kWh}$ of electricity. Projections for 20032030 are provided in the table below. Figure B-6 shows the amount of natural gas displaced annually.

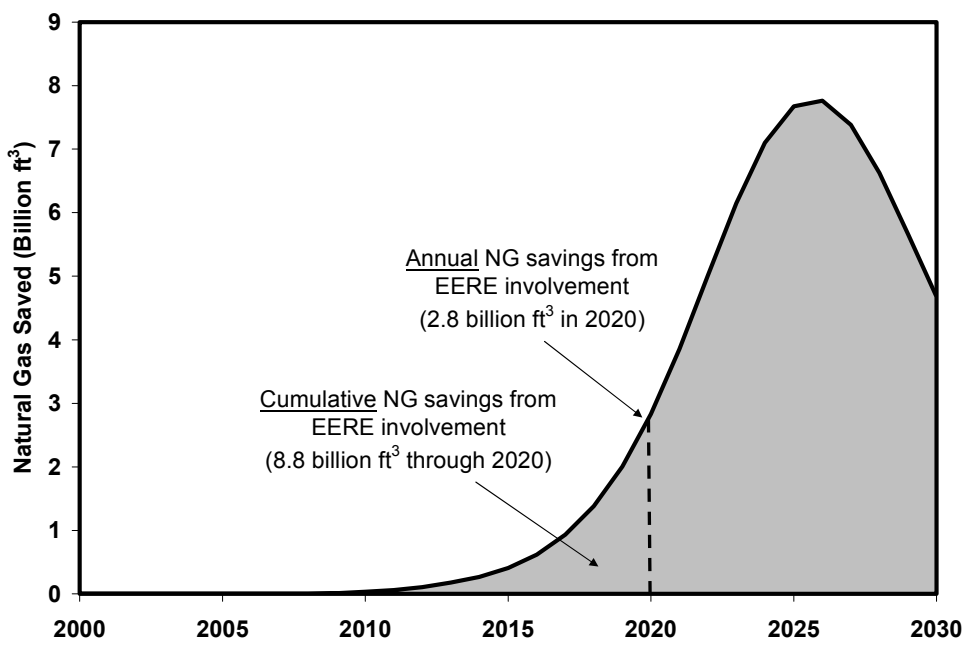

Figure B-6. Natural gas displaced from technology with 5-year accelerated market introduction 
Annual Direct Energy Displaced

\begin{tabular}{|l|c|c|c|c|c|c|c|c|c|c|}
\cline { 2 - 10 } Step (2) & 2003 & 2004 & 2005 & 2006 & 2007 & 2010 & 2015 & 2020 & 2025 & 2030 \\
\hline \begin{tabular}{|l} 
Direct Electricity Displaced \\
(billion kWhs)
\end{tabular} & 0.00 & 0.00 & 0.00 & 0.00 & 0.00 & 0.00 & 0.02 & 0.14 & 0.38 & 0.23 \\
\hline $\begin{array}{l}\text { Direct Natural Gas Displaced } \\
\text { (billion cubic feet) }\end{array}$ & 0.00 & 0.00 & 0.00 & 0.00 & 0.00 & 0.03 & 0.41 & 2.83 & 7.67 & 4.68 \\
\hline
\end{tabular}




\section{Converting from Direct to Primary Energy Displaced}

The process for converting projections of direct energy displaced into a single total primary energy displaced metric involves four steps. These steps are displayed in the diagram below.

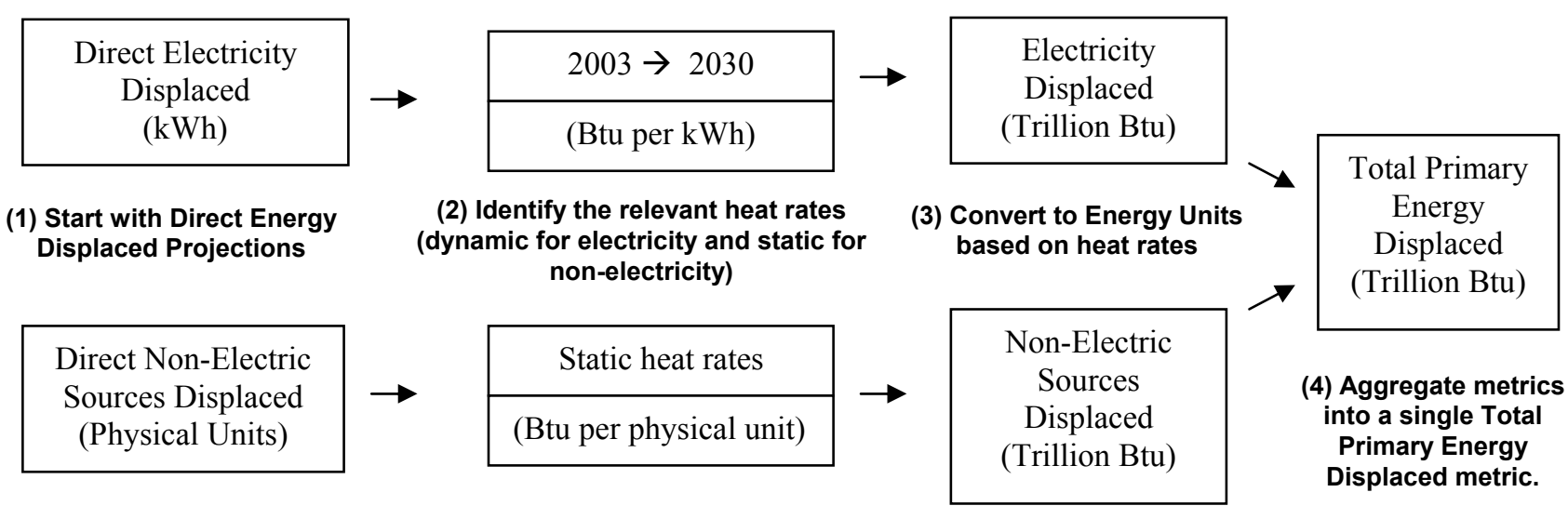

(1) The first step in the conversion process is to identify the electric and non-electric displaced energy projections. The direct electricity displaced projections will be expressed in kilowatthours; the direct non-electric projections will be expressed in barrels of oil, cubic feet of natural gas, and short tons of coal.

(2) The next step involves the conversion from direct units into heat content units using the heat rate of each direct fuel source.

\section{Electricity Heat Rates}

Electricity heat rates for GPRA2003 were derived by comparing the AEO2001 reference case against a side case in which electricity demand was reduced. The first step was to determine the marginal fuel mix based on the differences in $\mathrm{kWh}$ of electricity generated. The results, shown in the table below, indicate that EERE technologies are projected to displace electricity generated from fossil fuels.

\section{Projected Marginal Fuel Generation Mix}

(based on marginal kWh generated)

\begin{tabular}{|l|c|c|c|c|c|c|c|c|c|c|}
\hline Fuel & 2003 & 2004 & 2005 & 2006 & 2007 & 2010 & 2015 & 2020 & 2025 & 2030 \\
\hline Coal & $24.3 \%$ & $27.8 \%$ & $35.9 \%$ & $39.7 \%$ & $47.7 \%$ & $46.9 \%$ & $38.5 \%$ & $35.0 \%$ & $35.0 \%$ & $35.0 \%$ \\
\hline Natural Gas & $64.5 \%$ & $62.9 \%$ & $58.8 \%$ & $54.9 \%$ & $47.7 \%$ & $51.9 \%$ & $60.3 \%$ & $63.2 \%$ & $63.2 \%$ & $63.2 \%$ \\
\hline Oil & $11.3 \%$ & $9.2 \%$ & $5.3 \%$ & $5.4 \%$ & $4.7 \%$ & $1.2 \%$ & $1.3 \%$ & $1.8 \%$ & $1.8 \%$ & $1.8 \%$ \\
\hline
\end{tabular}


The second step was to calculate the marginal electricity heat rates for each fuel source based on a comparison of the two cases. The results are shown in the table below.

\section{Projected Marginal Electricity Heat Rates by Fuel Source}

\begin{tabular}{|l|r|r|r|r|r|r|r|r|r|r|}
\hline Projected Electricity Heat Rates & 2003 & 2004 & 2005 & 2006 & 2007 & 2010 & 2015 & 2020 & 2025 & 2030 \\
\hline Coal (Btu per kWh) & 10,724 & 10,616 & 10,601 & 10,583 & 10,556 & 10,387 & 10,402 & 9,942 & 9,942 & 9,942 \\
\hline Natural Gas (Btu per kWh) & 10,858 & 10,787 & 10,573 & 9,736 & 9,624 & 7,754 & 6,866 & 6,705 & 6,705 & 6,705 \\
\hline Oil (Btu per kWh) & 10,599 & 10,494 & 10,767 & 10,724 & 10,354 & 10,354 & 10,043 & 9,658 & 9,658 & 9,658 \\
\hline
\end{tabular}

To derive the dynamic GPRA2003 electricity heat rates, the percentage of the marginal mix associated with each fuel source was multiplied by the expected electricity heat rate for the same source. This yielded the intermediate apportioned heat content associated with each generation source. Then, for each forecast year, the apportioned heat contents were summed to arrive at a final GPRA2003 heat rate. For example, in the year 2020, electricity generated from coal is expected to account for 35.0 percent of the marginal mix, electricity generated from natural gas 63.2 percent, and oil 1.8 percent. The expected electricity heat rates in 2020 for coal, natural gas and oil are 9,942 and 6,705 and 9,658 Btu/kWh, respectively. Therefore, the GPRA2003 heat rate for 2020 is $(35.0 \%)(9,942)+(63.2 \%)(6,705)+(1.8 \%)(9,658)=7,891 \mathrm{Btu} / \mathrm{kWh}$.

\section{GPRA2003 Electricity Heat Rates}

\begin{tabular}{|c|c|c|c|c|c|c|c|c|c|c|}
\hline GPRA2003 Heat Rate & 2003 & 2004 & 2005 & 2006 & 2007 & 2010 & 2015 & 2020 & 2025 & 2030 \\
\hline Electricity (Btu per kWh) & 10,796 & 10,713 & 10,593 & 10,126 & 10,102 & 9,019 & 8,266 & 7,891 & 7,891 & 7,891 \\
\hline
\end{tabular}




\section{Non-Electric Heat Rates}

The heat rates used for conversion of non-electric sources are much more straightforward. The table below contains the appropriate conversion factors for these sources that are based on heat rate estimates provided in AEO2001 Table H1. Simply find the matching direct energy displaced source with the appropriate heat rate from the table below.

\section{GPRA2003 Non-Electricity Heat Rates}

\begin{tabular}{|c|c|c|c|}
\hline \multicolumn{4}{|l|}{ Coal } \\
\hline & Coal Production & million Btu per short ton & 21.224 \\
\hline & Coal Consumption & million Btu per short ton & 20.760 \\
\hline & Coke Plants & million Btu per short ton & 26.800 \\
\hline & Industrial & million Btu per short ton & 22.104 \\
\hline & Residential and Commercial & million Btu per short ton & 22.783 \\
\hline & Electric Utilities & million Btu per short ton & 20.479 \\
\hline & Coal Coke & million Btu per short ton & 24.800 \\
\hline \multicolumn{4}{|c|}{ 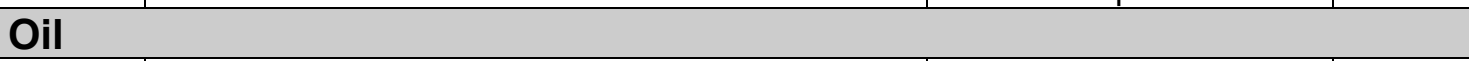 } \\
\hline & Crude Oil Production & million Btu per barrel & 5.800 \\
\hline & Oil Products Consumption & million Btu per barrel & 5.360 \\
\hline & Motor Gasoline & million Btu per barrel & 5.234 \\
\hline & Jet Fuel (Kerosene) & million Btu per barrel & 5.670 \\
\hline & Distillate Fuel Oil & million Btu per barrel & 5.825 \\
\hline & Residual Fuel Oil & million Btu per barrel & 6.287 \\
\hline & Liquefied Petroleum Gas & million Btu per barrel & 3.625 \\
\hline & Kerosene & million Btu per barrel & 5.670 \\
\hline & Petrochemical Feedstocks & million Btu per barrel & 5.630 \\
\hline & Unfinished Oils & million Btu per barrel & 5.825 \\
\hline \multicolumn{4}{|c|}{ Natural Gas } \\
\hline & Natural Gas Production & Btu per cubic foot & 1,026 \\
\hline & Natural Gas Consumption & Btu per cubic foot & 1,026 \\
\hline & Non-electric Utilities & Btu per cubic foot & 1,027 \\
\hline & Electric Utilities & Btu per cubic foot & 1,019 \\
\hline
\end{tabular}

Source: AEO2001, Table H1.

(3) The third step involves multiplying the above heat rates by the direct energy displaced projections.

(4) The final step is to sum the energy displaced estimates (not expressed in heat content units) for each forecast year. 


\section{Converting from Direct to Primary Energy - An Example}

To better understand the mechanics of the energy conversion process, consider the following example, which continues from the amount of annual direct energy displaced calculated above.

\begin{tabular}{|l|c|c|c|c|c|c|c|c|c|c|}
\cline { 2 - 10 } Step (1) & 2003 & 2004 & 2005 & 2006 & 2007 & 2010 & 2015 & 2020 & 2025 & 2030 \\
\hline $\begin{array}{l}\text { Direct Electricity Displaced } \\
\text { (billion kWhs) }\end{array}$ & 0.00 & 0.00 & 0.00 & 0.00 & 0.00 & 0.00 & 0.02 & 0.14 & 0.38 & 0.23 \\
\hline $\begin{array}{l}\text { Direct Natural Gas Displaced } \\
\text { (billion cubic feet) }\end{array}$ & 0.00 & 0.00 & 0.00 & 0.00 & 0.00 & 0.03 & 0.41 & 2.83 & 7.67 & 4.68 \\
\hline
\end{tabular}

In step 2, the relevant heat rates are identified. A static conversion factor is used for the nonelectric projections while a dynamic heat rate is used for electricity.

\begin{tabular}{|l|c|c|c|c|c|c|c|c|c|c|}
\cline { 2 - 10 } Step (2) & 2003 & 2004 & 2005 & 2006 & 2007 & 2010 & 2015 & 2020 & 2025 & 2030 \\
\hline $\begin{array}{l}\text { Electricity } \\
\text { (Btu per kWh) }\end{array}$ & 10796 & 10713 & 10593 & 10126 & 10102 & 9019 & 8266 & 7891 & 7891 & 7891 \\
\hline $\begin{array}{l}\text { Natural Gas Consumption } \\
\text { (Btu per cubic foot) }\end{array}$ & 1026 & 1026 & 1026 & 1026 & 1026 & 1026 & 1026 & 1026 & 1026 & 1026 \\
\hline
\end{tabular}

In step 3, all direct energy displaced estimates are converted from physical units to heat content units by multiplying by the appropriate heat rates.

\begin{tabular}{|l|c|c|c|c|c|c|c|c|c|c|}
\cline { 2 - 10 } Step (3) & 2003 & 2004 & 2005 & 2006 & 2007 & 2010 & 2015 & 2020 & 2025 & 2030 \\
\hline Electricity (trillion Btu) & 0.00 & 0.00 & 0.00 & 0.00 & 0.00 & 0.01 & 0.17 & 1.12 & 3.03 & 1.84 \\
\hline Natural Gas (trillion Btu) & 0.00 & 0.00 & 0.00 & 0.00 & 0.00 & 0.03 & 0.42 & 2.90 & 7.87 & 4.80 \\
\hline
\end{tabular}

Step 4 simply involves summing the metrics in each forecast year to arrive at projections of annual primary energy displaced.

\begin{tabular}{|l|c|c|c|c|c|c|c|c|c|c|}
\cline { 2 - 10 } Step (4) & 2003 & 2004 & 2005 & 2006 & 2007 & 2010 & 2015 & 2020 & 2025 & 2030 \\
\hline $\begin{array}{l}\text { Annual Primary Energy } \\
\text { Displaced (trillion Btu) }\end{array}$ & 0.00 & 0.00 & 0.00 & 0.00 & 0.00 & 0.05 & 0.59 & 4.02 & 10.90 & 6.64 \\
\hline
\end{tabular}

Step 5 involves calculating the cumulative primary energy displaced. This is simply a summation of annual energy benefits over the years of interest.

\begin{tabular}{|l|c|c|c|c|c|c|c|c|c|c|}
\cline { 2 - 10 } Step (5) & 2003 & 2004 & 2005 & 2006 & 2007 & 2010 & 2015 & 2020 & 2025 & 2030 \\
\hline $\begin{array}{l}\text { Cumulative Primary Energy } \\
\text { Displaced (trillion Btu) }\end{array}$ & 0.00 & 0.00 & 0.00 & 0.00 & 0.00 & 0.07 & 1.55 & 12.68 & 54.99 & 100.60 \\
\hline
\end{tabular}

Step 6 calculates the lifecycle primary energy displaced. This is a summation of the annual energy displaced over the lifetime of the technologies installed through that year.

\begin{tabular}{|l|c|c|c|c|c|c|c|c|c|c|}
\cline { 2 - 10 } Step (6) & 2003 & 2004 & 2005 & 2006 & 2007 & 2010 & 2015 & 2020 & 2025 & 2030 \\
\hline $\begin{array}{l}\text { Lifecycle Primary Energy } \\
\text { Displaced (trillion Btu) }\end{array}$ & 0.00 & 0.00 & 0.00 & 0.00 & 0.00 & 0.46 & 5.89 & 40.80 & 114.94 & 107.22 \\
\hline
\end{tabular}




\section{Calculating Emissions Displaced}

The methodology for calculating the level of emissions displaced continues from the conversion of direct to primary energy displaced. Emission factors are applied to the projections of primary energy displaced by energy source to obtain emissions displaced by energy source. These estimates are then summed to arrive at a final estimate of emissions displaced.

The GPRA2003 Data Call uses carbon emission factors found in Table 2 of the Assumptions to the AEO2001. These emission factors are based on the carbon content of the fuel and the fraction of the fuel consumed in combustion. The emission factors are based on 1999 data.

Adjusted Carbon Emission Factors (1999)

(Million Metric Tons of Carbon per Trillion Btu)

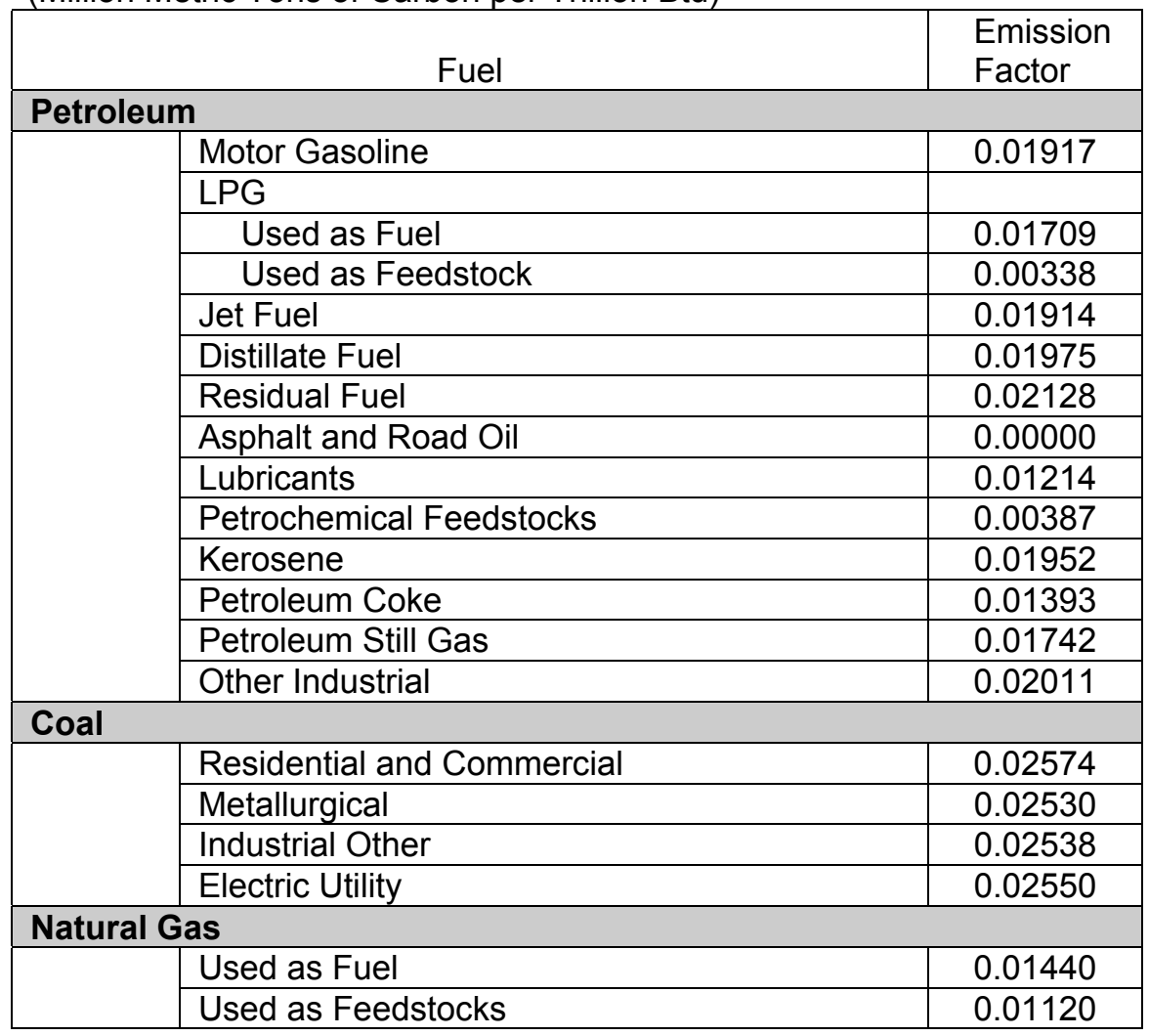

Like the electricity heat rate, the carbon emission factor for electricity changes over the forecast period with the changing projections of the marginal fuel mix. The petroleum mix for the utility sector is $95 \%$ residual fuel and $5 \%$ distillate fuel.

\section{Electricity Carbon Emission Factor (million metric tons of carbon per trillion Btu)}

\begin{tabular}{|l|r|r|r|r|r|r|r|r|r|r|}
\hline Carbon Coefficient & 2003 & 2004 & 2005 & 2006 & 2007 & 2010 & 2015 & 2020 & 2025 & 2030 \\
\hline Electricity (MMTCE per trillion Btu) & 0.01783 & 0.01808 & 0.01875 & 0.01939 & 0.02025 & 0.02048 & 0.01988 & 0.01944 & 0.01944 & 0.01944 \\
\hline
\end{tabular}


The Environmental Protection Agency catalogues criteria pollutant emission factors for numerous technologies. For the GPRA Data Call more generic emission factors have been calculated from aggregate emission and energy consumption data provided by EPA for 1997. These are provided in the tables below. Emission factors for specific technologies may be obtained from EPA's Compilation of Air Pollutant Emission Factors (AP-42) available on the world wide web at http://www.epa.gov/ttn/chief/ap42etc.html

\section{Emission Factors of Criteria Pollutants}

(Metric tonnes of emissions per trillion Btu)

\begin{tabular}{|l|c|c|c|c|c|}
\hline Fuel & NOx & SO2 & VOCs & CO & PM10 \\
\hline Coal & 254 & 568 & 1 & 11 & 12 \\
\hline Natural Gas & 106 & 0 & 3 & 29 & 0 \\
\hline Oil & 140 & 527 & 4 & 13 & 7 \\
\hline
\end{tabular}

Like the electricity carbon emission factor, the electricity criteria pollutant emission factors are dynamic, changing over time with the changing fuel mix.

\section{Electricity Emission Factors of Criteria Pollutants}

(Metric tonnes of emissions per trillion Btu)

\begin{tabular}{|l|c|c|c|c|c|c|c|c|c|c|}
\hline Criteria Pollutant & 2003 & 2004 & 2005 & 2006 & 2007 & 2010 & 2015 & 2020 & 2025 & 2030 \\
\hline NOx & 146 & 150 & 161 & 170 & 182 & 187 & 178 & 172 & 172 & 172 \\
\hline SO2 & 196 & 205 & 233 & 266 & 308 & 314 & 283 & 262 & 262 & 262 \\
\hline VOCs & 3 & 3 & 2 & 2 & 2 & 2 & 2 & 2 & 2 & 2 \\
\hline CO & 23 & 23 & 22 & 21 & 19 & 19 & 20 & 21 & 21 & 21 \\
\hline PM10 & 4 & 4 & 5 & 5 & 6 & 7 & 6 & 6 & 6 & 6 \\
\hline
\end{tabular}

\section{Calculating Emissions Displaced - An Example}

To better understand how to calculate emissions displaced, consider the following example for carbon. The example continues from primary energy displaced in the example above.

\begin{tabular}{|l|c|c|c|c|c|c|c|c|c|c|}
\cline { 2 - 10 } \multicolumn{1}{|c|}{ Step (1) } & 2003 & 2004 & 2005 & 2006 & 2007 & 2010 & 2015 & 2020 & 2025 & 2030 \\
\hline Electricity (trillion Btu) & 0.00 & 0.00 & 0.00 & 0.00 & 0.00 & 0.01 & 0.17 & 1.12 & 3.03 & 1.84 \\
\hline Natural Gas (trillion Btu) & 0.00 & 0.00 & 0.00 & 0.00 & 0.00 & 0.03 & 0.42 & 2.90 & 7.87 & 4.80 \\
\hline
\end{tabular}

Instead of summing these metrics to arrive at annual primary energy displaced, the appropriate carbon emissions coefficients are applied.

\begin{tabular}{|l|c|c|c|c|c|c|c|c|c|c|}
\cline { 2 - 9 } Step (2) & 2003 & 2004 & 2005 & 2006 & 2007 & 2010 & 2015 & 2020 & 2025 & 2030 \\
\hline $\begin{array}{l}\text { Electricity } \\
\text { (MMTCE per trillion Btu) }\end{array}$ & 0.01783 & 0.01808 & 0.01875 & 0.01939 & 0.02025 & 0.02048 & 0.01988 & 0.01944 & 0.01944 & 0.01944 \\
\hline $\begin{array}{l}\text { Natural Gas } \\
\text { (MMTCE per trillion Btu) }\end{array}$ & 0.01440 & 0.01440 & 0.01440 & 0.01440 & 0.01440 & 0.01440 & 0.01440 & 0.01440 & 0.01440 & 0.01440 \\
\hline
\end{tabular}


The resultant emissions displaced projections are listed in the table below.

\begin{tabular}{|l|c|c|c|c|c|c|c|c|c|c|}
\cline { 2 - 10 } Step (3) & 2003 & 2004 & 2005 & 2006 & 2007 & 2010 & 2015 & 2020 & 2025 & 2030 \\
\hline Electricity (MMTCE) & 0.00 & 0.00 & 0.00 & 0.00 & 0.00 & 0.00 & 0.00 & 0.02 & 0.06 & 0.04 \\
\hline Natural Gas (MMTCE) & 0.00 & 0.00 & 0.00 & 0.00 & 0.00 & 0.00 & 0.01 & 0.04 & 0.11 & 0.07 \\
\hline
\end{tabular}

The next step is to sum these individual estimates to arrive at annual carbon equivalent emissions displaced as illustrated below.

\begin{tabular}{|l|c|c|c|c|c|c|c|c|c|c|}
\cline { 2 - 9 } Step (4) & 2003 & 2004 & 2005 & 2006 & 2007 & 2010 & 2015 & 2020 & 2025 & 2030 \\
\hline \begin{tabular}{|l} 
Annual Carbon Equivalent \\
Emissions Displaced \\
(MMTons)
\end{tabular} & 0.00 & 0.00 & 0.00 & 0.00 & 0.00 & 0.00 & 0.01 & 0.06 & 0.17 & 0.10 \\
\hline
\end{tabular}

The next step is to sum these individual estimates to arrive at cumulative carbon equivalent emissions displaced as illustrated below.

\begin{tabular}{|l|c|c|c|c|c|c|c|c|c|c|}
\cline { 2 - 10 } Step (5) & 2003 & 2004 & 2005 & 2006 & 2007 & 2010 & 2015 & 2020 & 2025 & 2030 \\
\hline $\begin{array}{l}\text { Cumulative Carbon } \\
\text { Equivalent Emissions } \\
\text { Displaced (MMTons) }\end{array}$ & 0.00 & 0.00 & 0.00 & 0.00 & 0.00 & 0.00 & 0.02 & 0.20 & 0.87 & 1.59 \\
\hline
\end{tabular}

The cumulative life cycle carbon emissions displaced are also calculated.

\begin{tabular}{|l|c|c|c|c|c|c|c|c|c|c|}
\cline { 2 - 9 } Step (6) & 2003 & 2004 & 2005 & 2006 & 2007 & 2010 & 2015 & 2020 & 2025 & 2030 \\
\hline $\begin{array}{l}\text { Cumulative Life Cycle Carbon } \\
\text { Equivalent Emissions } \\
\text { Displaced (MMTons) }\end{array}$ & 0.00 & 0.00 & 0.00 & 0.00 & 0.00 & 0.01 & 0.09 & 0.65 & 1.82 & 1.70 \\
\hline
\end{tabular}




\section{Calculating Energy Cost Savings}

The methodology for calculating energy cost savings differs slightly for electricity and nonelectricity energy sources. For non-electricity energy sources, sector energy prices are applied to the projections of primary energy displaced by energy source to obtain energy cost savings by energy source. For electricity, the amount of direct electricity displaced in $\mathrm{kWh}$ is converted to site electricity in trillion btu and then multiplied by the sector electricity price. Electricity and non-electricity cost savings estimates are then summed to arrive at total energy cost savings. The GPRA2003 Data Call uses energy prices in Table 20 of the Assumptions to the AEO2001 (found in Appendix $\mathrm{C}$ of the data call).

\section{Calculating Energy Cost Savings - An Example}

To better understand how to calculate energy cost savings, consider the following example. The process starts with the estimates of direct energy displaced.

\begin{tabular}{|l|c|c|c|c|c|c|c|c|c|c|}
\cline { 2 - 10 } Step (1) & 2003 & 2004 & 2005 & 2006 & 2007 & 2010 & 2015 & 2020 & 2025 & 2030 \\
\hline $\begin{array}{l}\text { Direct Electricity Displaced } \\
\text { (billion kWhs) }\end{array}$ & 0.00 & 0.00 & 0.00 & 0.00 & 0.00 & 0.00 & 0.02 & 0.14 & 0.38 & 0.23 \\
\hline $\begin{array}{l}\text { Direct Natural Gas Displaced } \\
\text { (billion cubic feet) }\end{array}$ & 0.00 & 0.00 & 0.00 & 0.00 & 0.00 & 0.03 & 0.41 & 2.83 & 7.67 & 4.68 \\
\hline
\end{tabular}

Heat rates are applied to the estimates. Note that an electricity consumption heat rate is used.

\begin{tabular}{|l|c|c|c|c|c|c|c|c|c|c|}
\cline { 2 - 9 } Step (2) & 2003 & 2004 & 2005 & 2006 & 2007 & 2010 & 2015 & 2020 & 2025 & 2030 \\
\hline $\begin{array}{l}\text { Electricity Consumption } \\
\text { (Btu per kWh) }\end{array}$ & 3412 & 3412 & 3412 & 3412 & 3412 & 3412 & 3412 & 3412 & 3412 & 3412 \\
\hline $\begin{array}{l}\text { Natural Gas Consumption } \\
\text { (Btu per cubic foot) }\end{array}$ & 1026 & 1026 & 1026 & 1026 & 1026 & 1026 & 1026 & 1026 & 1026 & 1026 \\
\hline
\end{tabular}

This results in the heat content for each energy source.

\begin{tabular}{|l|c|c|c|c|c|c|c|c|c|c|}
\cline { 2 - 11 } Step (3) & 2003 & 2004 & 2005 & 2006 & 2007 & 2010 & 2015 & 2020 & 2025 & 2030 \\
\hline Site Electricity (trillion Btu) & 0.00 & 0.00 & 0.00 & 0.00 & 0.00 & 0.01 & 0.07 & 0.48 & 1.31 & 0.80 \\
\hline Natural Gas (trillion Btu) & 0.00 & 0.00 & 0.00 & 0.00 & 0.00 & 0.03 & 0.42 & 2.90 & 7.87 & 4.80 \\
\hline
\end{tabular}

Appropriate energy prices are applied (industrial sector prices are used here).

\begin{tabular}{|l|c|c|c|c|c|c|c|c|c|c|}
\cline { 2 - 10 } Step (4) & 2003 & 2004 & 2005 & 2006 & 2007 & 2010 & 2015 & 2020 & 2025 & 2030 \\
\hline $\begin{array}{l}\text { Electricity } \\
(1999 \text { \$ per million Btu) }\end{array}$ & 12.94 & 12.83 & 12.34 & 12.03 & 11.61 & 11.24 & 11.27 & 11.62 & 11.82 & 12.02 \\
\hline $\begin{array}{l}\text { Natural Gas } \\
(1999 \text { \$ per million Btu) }\end{array}$ & 3.24 & 3.14 & 3.17 & 3.20 & 3.24 & 3.31 & 3.45 & 3.76 & 4.01 & 4.28 \\
\hline
\end{tabular}


Resulting in energy cost savings projections for each fuel.

\begin{tabular}{|l|c|c|c|c|c|c|c|c|c|c|}
\cline { 2 - 10 } \multicolumn{1}{c|}{ Step (5) } & 2003 & 2004 & 2005 & 2006 & 2007 & 2010 & 2015 & 2020 & 2025 & 2030 \\
\hline Electricity (million 1999 \$) & 0 & 0 & 0 & 0 & 0 & 0 & 1 & 6 & 15 & 10 \\
\hline Natural Gas (million 1999 \$) & 0 & 0 & 0 & 0 & 0 & 0 & 1 & 11 & 32 & 21 \\
\hline
\end{tabular}

Individual fuel savings estimates are summed to arrive at annual energy cost savings.

\begin{tabular}{|l|c|c|c|c|c|c|c|c|c|c|}
\cline { 2 - 10 } Step (6) & 2003 & 2004 & 2005 & 2006 & 2007 & 2010 & 2015 & 2020 & 2025 & 2030 \\
\hline \begin{tabular}{|l} 
Annual Energy Cost Savings \\
(million 1999 \$)
\end{tabular} & 0 & 0 & 0 & 0 & 0 & 0 & 2 & 17 & 47 & 30 \\
\hline
\end{tabular}

The annual savings are summed to arrive at an estimate of cumulative energy cost savings.

Step (7)

Cumulative Energy Cost

Savings (million 1999 \$)

\begin{tabular}{|c|c|c|c|c|c|c|c|c|c|}
\hline 2003 & 2004 & 2005 & 2006 & 2007 & 2010 & 2015 & 2020 & 2025 & 2030 \\
\hline 0 & 0 & 0 & 0 & 0 & 0 & 6 & 50 & 230 & 432 \\
\hline
\end{tabular}

Cumulative life cycle energy cost savings are then calculated.

\begin{tabular}{|l|c|c|c|c|c|c|c|c|c|c|}
\cline { 2 - 10 } Step (8) & 2003 & 2004 & 2005 & 2006 & 2007 & 2010 & 2015 & 2020 & 2025 & 2030 \\
\hline $\begin{array}{l}\text { Cumulative Life Cycle } \\
\text { Energy Cost Savings } \\
\text { (million 1999 \$) }\end{array}$ & 0 & 0 & 0 & 0 & 0 & 2 & 23 & 171 & 497 & 462 \\
\hline
\end{tabular}




\section{Calculating Non-Energy Cost Savings}

In certain instances an advanced EERE technology will result in cost savings beyond those derived from decreased energy consumption. For instance, some technologies may result in productivity gains to industry. To the extent possible, programs should identify and estimate these non-energy cost savings.

\section{Calculating Non-Energy Cost Savings - An Example}

The advanced EERE technology in the above example results in less down-time for an industrial process. As a result, an additional $\$ 250,000$ in goods are produced annually for each unit that is installed. The productivity savings are estimated to remain constant for the foreseeable future. Per unit productivity savings are applied to the market penetration levels identified above to arrive at annual non-energy cost savings.

\begin{tabular}{|l|c|c|c|c|c|c|c|c|c|c|}
\cline { 2 - 10 } Step (1) & 2003 & 2004 & 2005 & 2006 & 2007 & 2010 & 2015 & 2020 & 2025 & 2030 \\
\hline $\begin{array}{l}\text { Annual Non-Energy Cost } \\
\text { Savings (million 1999 \$) }\end{array}$ & 0 & 0 & 0 & 0 & 0 & 0 & 3 & 23 & 62 & 31 \\
\hline
\end{tabular}

The annual figures are summed to arrive at a cumulative estimate of non-energy cost savings.

\begin{tabular}{|l|c|c|c|c|c|c|c|c|c|c|}
\cline { 2 - 10 } Step (2) & 2003 & 2004 & 2005 & 2006 & 2007 & 2010 & 2015 & 2020 & 2025 & 2030 \\
\hline $\begin{array}{l}\text { Cumulative Non-Energy Cost } \\
\text { Savings (million 1999 \$) }\end{array}$ & 0 & 0 & 0 & 0 & 0 & 0 & 8 & 70 & 312 & 553 \\
\hline
\end{tabular}

Cumulative life cycle non-energy cost savings are then calculated

\begin{tabular}{|l|c|c|c|c|c|c|c|c|c|c|}
\cline { 2 - 10 } Step (3) & 2003 & 2004 & 2005 & 2006 & 2007 & 2010 & 2015 & 2020 & 2025 & 2030 \\
\hline $\begin{array}{l}\text { Cumulative Life Cycle } \\
\begin{array}{l}\text { Non-Energy Cost Savings } \\
\text { (million 1999 \$) }\end{array}\end{array}$ & 0 & 0 & 0 & 0 & 0 & 2 & 32 & 230 & 651 & 542 \\
\hline
\end{tabular}




\section{Calculating Consumer Investment}

Technology cost and market penetration information should be used to calculate the consumer investment in the advanced EERE technology.

\section{Calculating Consumer Investment - An Example}

In the first step the incremental annualized capital costs and incremental O\&M costs are taken from the example above.

\section{Incremental Annualized Capital Costs (Millions 1999 \$)}

\begin{tabular}{|l|c|c|c|c|c|c|c|c|c|c|}
\cline { 2 - 10 } \multicolumn{1}{l|}{ Step (1a) } & 2003 & 2004 & 2005 & 2006 & 2007 & 2010 & 2015 & 2020 & 2025 & 2030 \\
\hline Adv. Tech. With EERE & --- & --- & --- & -- & -- & 0.11 & 0.09 & 0.07 & 0.05 & 0.04 \\
\hline Adv. Tech. Without EERE & --- & --- & --- & --- & --- & -- & 0.13 & 0.11 & 0.09 & 0.07 \\
\hline
\end{tabular}

\section{Incremental O\&M Costs (Millions 1999 \$)}

\begin{tabular}{|l|c|c|c|c|c|c|c|c|c|c|}
\cline { 2 - 9 } \multicolumn{1}{l|}{ Step (1b) } & 2003 & 2004 & 2005 & 2006 & 2007 & 2010 & 2015 & 2020 & 2025 & 2030 \\
\hline Adv. Tech. With EERE & --- & --- & -- & -- & --- & 0.0107 & 0.0087 & 0.0068 & 0.0052 & 0.0037 \\
\hline Adv. Tech. Without EERE & --- & --- & --- & --- & --- & -- & 0.0134 & 0.0112 & 0.0092 & 0.0074 \\
\hline
\end{tabular}

Annual market penetration estimates are also taken from the example above.

\section{Annual Market Penetration (\# units)}

\begin{tabular}{|l|c|c|c|c|c|c|c|c|c|c|}
\cline { 2 - 11 } \multicolumn{1}{l|}{ Step (2) } & 2003 & 2004 & 2005 & 2006 & 2007 & 2010 & 2015 & 2020 & 2025 & 2030 \\
\hline Adv. Tech. With EERE & --- & --- & --- & --- & --- & 1 & 5 & 32 & 49 & 11 \\
\hline Adv. Tech. Without EERE & --- & --- & --- & --- & --- & --- & 1 & 5 & 32 & 49 \\
\hline
\end{tabular}

The incremental annualized capital and O\&M costs are then multiplied by the annual number of units installed to arrive at incremental consumer investment. For instance, the incremental capital investment for the 32 units added in 2020 would be about $\$ 1.6$ million. This is a combination of three calculations. First, there is $\$ 2.2$ million in investment for the 32 units relative to the next best alternative (32 units $\mathrm{x} \$ 0.07$ million/unit). Second, there is $\$ 0.6$ million less invested because 5 of the units would have been installed without EERE involvement ( 5 units $\mathrm{x} \$ 0.11 \mathrm{million} / \mathrm{unit})$. Third, there is also a negligible reduction for stock being turned over at a lower cost (1 unit $\mathrm{x} \$ 0.04$ million). The $\$ 1.6$ million represents additional capital 
investment consumers will need to make in 2020 (assuming capital costs are annualized). Finally, there are \$0.2 million in additional O\&M costs associated with the 32 new technologies.

The incremental capital and O\&M investments are then summed to arrive at $\$ 1.8$ million in incremental consumer investment. The incremental consumer investment is positive in 2030 because the market penetration curve of the advanced technology with EERE involvement is slowing down relative to the market penetration of the advanced technology without EERE involvement.

\section{Incremental Consumer Investment}

\begin{tabular}{|l|c|c|c|c|c|c|c|c|c|c|}
\cline { 2 - 10 } \multicolumn{1}{l|}{ Step (3) } & 2003 & 2004 & 2005 & 2006 & 2007 & 2010 & 2015 & 2020 & 2025 & 2030 \\
\hline $\begin{array}{l}\text { Incremental Capital } \\
\text { Investment (Millions 1999 \$) }\end{array}$ & 0 & 0 & 0 & 0 & 0 & 0 & 0 & -2 & -1 & 4 \\
\hline $\begin{array}{l}\text { Incremental O\&M } \\
\text { Investment (Millions 1999 \$) }\end{array}$ & 0 & 0 & 0 & 0 & 0 & 0 & 0 & 0 & 0 & 0 \\
\hline $\begin{array}{l}\text { Incremental Consumer } \\
\text { Investment (Millions 1999 \$) }\end{array}$ & 0 & 0 & 0 & 0 & 0 & 0 & 0 & -2 & -1 & 4 \\
\hline
\end{tabular}

Annual consumer investment is the summation of incremental consumer investment.

\section{Annual Consumer Investment}

\begin{tabular}{|c|c|c|c|c|c|c|c|c|c|c|}
\hline Step (4) & 2003 & 2004 & 2005 & 2006 & 2007 & 2010 & 2015 & 2020 & 2025 & 2030 \\
\hline $\begin{array}{l}\text { Annual Capital Investment } \\
\text { (Millions 1999 \$) }\end{array}$ & 0 & 0 & 0 & 0 & 0 & 0 & -1 & -6 & -11 & 6 \\
\hline $\begin{array}{l}\text { Annual O\&M } \\
\text { Investment (Millions } 1999 \text { \$) }\end{array}$ & 0 & 0 & 0 & 0 & 0 & 0 & 0 & -1 & -1 & 1 \\
\hline $\begin{array}{l}\begin{array}{l}\text { Annual Consumer Investment } \\
\text { (Millions 1999 \$) }\end{array} \\
\end{array}$ & 0 & 0 & 0 & 0 & 0 & 0 & -1 & -7 & -12 & 6 \\
\hline
\end{tabular}

Cumulative consumer investment is simply a summation of incremental consumer investment to date.

\section{Cumulative Consumer Investment}

\begin{tabular}{|l|c|c|c|c|c|c|c|c|c|c|}
\cline { 2 - 10 } \multicolumn{1}{l|}{ Step (5) } & 2003 & 2004 & 2005 & 2006 & 2007 & 2010 & 2015 & 2020 & 2025 & 2030 \\
\hline $\begin{array}{l}\text { Cumulative Capital } \\
\text { Investment (Millions 1999 \$) }\end{array}$ & 0 & 0 & 0 & 0 & 0 & 0 & -3 & -21 & -74 & -85 \\
\hline $\begin{array}{l}\text { Cumulative O\&M } \\
\text { Investment (Millions 1999 \$) }\end{array}$ & 0 & 0 & 0 & 0 & 0 & 0 & 0 & -2 & -7 & -8 \\
\hline $\begin{array}{l}\text { Cumulative Consumer } \\
\text { Investment (Millions 1999 \$) }\end{array}$ & 0 & 0 & 0 & 0 & 0 & 0 & -3 & -24 & -81 & -93 \\
\hline
\end{tabular}

Cumulative lifecycle consumer investment is then calculated. 


\section{Cumulative Lifecycle Consumer Investment}

\begin{tabular}{|l|c|c|c|c|c|c|c|c|c|c|}
\cline { 2 - 10 } \multicolumn{1}{|c|}{ Step (6) } & 2003 & 2004 & 2005 & 2006 & 2007 & 2010 & 2015 & 2020 & 2025 & 2030 \\
\hline $\begin{array}{l}\text { Cumulative Lifecycle } \\
\begin{array}{l}\text { Capital Investment } \\
\text { (Millions 1999 \$) }\end{array}\end{array}$ & 0 & 0 & 0 & 0 & 0 & -1 & -11 & -64 & -124 & -6 \\
\hline $\begin{array}{l}\text { Cumulative Lifecycle } \\
\text { O\&M Investment } \\
\text { (Millions 1999 \$) }\end{array}$ & 0 & 0 & 0 & 0 & 0 & 0 & -1 & -6 & -12 & -1 \\
\hline $\begin{array}{l}\text { Cumulative Lifecycle } \\
\text { Consumer Investment } \\
\text { (Millions 1999 \$) }\end{array}$ & 0 & 0 & 0 & 0 & 0 & -1 & -13 & -70 & -137 & -6 \\
\hline
\end{tabular}




\section{Calculating Net Economic Benefits}

The net economic benefits of the advanced technology is a summation of public and private expenditures, energy cost savings, non-energy cost savings, and consumer investment. Calculations for annual, cumulative, and life cycle benefits will be performed by OPBM. 


\section{Appendix C Diffusion Curves}




\section{Appendix C \\ Technology Diffusion Models - Application to Selected Energy-Efficient Products for Buildings}

\section{Abstract}

Diffusion models represent the principal forecasting method for determining potential market penetration for products that have not yet been introduced into the marketplace. Because this situation generally applies to the long-term forecasting horizon of technology assessment models, a means to credibly represent price and policy effects in diffusion models is a key factor in improving the usefulness of market assessment studies. The basic diffusion models assume that the cumulative market penetration follows a characteristic time path (usually in the form of an S-shaped curve).

Perhaps the dominant type of diffusion model is the mixed-influence model introduced by Bass (1969). The Bass model incorporates parameters that reflect both external (e.g., mass media communication) and internal influences (e.g., word of mouth). A study was conducted by Pacific Northwest National Laboratory (PNNL) to estimate Bass specification for ten selected energyefficient products in buildings to help assess technologies supported by the U.S. Department of Energy's (DOE's) Office of Building Technology, State and Community Programs (BTS). This appendix summarizes the results of that study.

\section{Scientific and Technical Approach}

A study was conducted by PNNL to examine the historical market penetration for ten energyefficient products related to the building sector. Diffusion models were estimated for each product, based on the specification proposed by Frank Bass (1969). The resulting models are intended to help assess technologies supported by DOE's BTS. This model development and empirical analysis are designed to generate more credible predictions of the adoption process of important energy-efficiency technologies in the buildings sector.

The basic Bass diffusion model, which is possibly the most widely used specification for analyzing market penetration, assumes that the potential market in which the new technology is penetrating is fixed. In reality, the potential market is usually growing in response to a falling price as the manufacturing process and industry structure behind the new technology evolve. This study is aimed toward developing a simple structural model that incorporates these effects and that can be easily estimated from historical data. Given a suitable conceptual model, its parameters can be estimated from data related to several energy technologies.

Most studies of technology adoption have focused either on defining the market potential of the new technology or on the pace by which the technology is adopted. Models that have integrated both aspects generally have not been subjected to historical validation of their underlying parameters. Therefore, in general, little empirical basis exists to suggest which process — diffusion or expanding market potential due to falling costs - might be more influential in driving the penetration of new technologies. 


\section{Background}

A report by the Electric Power Research Institute (EPRI) (1991) provides a good overview of market penetration approaches. Although the report has a slant toward utilities, much of the discussion applies to all types of energy-saving technologies. The report was prepared by the Research Triangle Institute in North Carolina.

The EPRI report clearly distinguishes between two aspects of the process for forecasting market penetration: 1) forecasting market potential, and 2) forecasting the rate of market penetration. Forecasting market potential can involve several different concepts of potential, including maximum, technical, and economic potential.

The EPRI report states that the factors affecting the rate of market penetration are predominantly different from factors affecting market potential. For example, comparative advantage — often determined by economic cost—strongly affects market potential. However, comparative advantage doesn't appear to have as strong an effect on the rate of market penetration.

In trying to distinguish the key factors affecting potential vs. penetration, EPRI suggests that market potential is predominantly influenced by the following:

- the market population and demographic trends

- the needs of the market: customer perceptions, attitudes, and beliefs

- feasibility of the product, which depends on functional characteristics of the product and its economic advantages compared with alternatives.

According to EPRI, the rate of market penetration is predominantly influenced by other factors:

1. marketing effort, such as promotion, advertising, and product positioning

2. product characteristics, such as complexity, compatibility, trialability, and observability

3. characteristics of potential adopters, such as decision-making style, innovativeness, and adoption processes

4. market characteristics, such as macroeconomic conditions, degree of social interaction among potential adopters, and competitive conditions.

Approaches to predicting the diffusion of a new technology general fall under the category of judgmental methods or model-based methods. The judgmental methods share the common trait that they don't require mathematical models or computations; they rely implicitly on the experience and perceptions of the forecaster. On the other hand, model-based methods use wellspecified algorithms to process and analyze data. Thus, the model-based methods can provide systematic forecasts of market penetration that are reproducible and amenable to being incorporated into broader integrated models.

Model-based methods can be divided into two major categories: extrapolation models and causal models. Extrapolation methods have the following: 1) naive diffusion process models, 2) moving average, 3) exponential smoothing, 4) Census Bureau X-11, 5) Box-Jenkins, and 6) Multivariate Time Series. 
Of the extrapolation methods, the diffusion models represent the principal method for dealing with products that have not yet been introduced. Because this situation generally applies to long-range models, the discussion will be restricted to these models.

The diffusion models assume that the cumulative market penetration follows a characteristic time path (usually in the form of an S-shaped curve). An apt analogy is the spread of contagious disease in a fixed population. Once begun, growth of the disease in the number of infected individuals may follow a stable, predictable path. The time path of the infection in the population depends on the probability of spontaneous infection, the share of infected individuals, and probability of uninfected individuals interacting with individuals already infected. The notion underlying the penetration rate models is that information about the new technology sufficient to induce its adoption - is similar to an infectious disease (although with a much more positive connotation). This model provides the rationale behind the S-shaped ("logistic") penetration curves that are often observed.

\section{Bass Diffusion Model}

Perhaps the dominant type of diffusion model is the mixed-influence model introduced by Bass in the late 1960s. This two-parameter model incorporates parameters that reflect both external and internal influences. The external influence (corresponding to the "spontaneous" infection mentioned above) is exemplified by mass media communication, size of sales force, or other structured channels of information. Spontaneous refers to the adopter not being influenced by previous adopters but by advertising or some other "external change-agent."

In contrast, the internal influence is intended to capture interpersonal communication or word of mouth (i.e., the contagious aspect of the disease analogy above). This also termed the "imitative effect"; the decision to adopt is made only after being influenced by prior adopters.

The basic specification of the Bass model is as follows:

$$
\mathrm{dN}(\mathrm{t}) / \mathrm{dt}=[\mathrm{p}+\mathrm{q} / \mathrm{MN}(\mathrm{t})][\mathrm{M}-\mathrm{N}(\mathrm{t})]
$$

where

$$
\begin{aligned}
\mathrm{N}(\mathrm{t}) & =\text { cumulative number of adoptions at time } \mathrm{t} \\
\mathrm{M} & =\text { market potential, a constant } \\
\mathrm{p} & =\text { the coefficient of innovation or external influence } \\
\mathrm{q} & =\text { the coefficient of imitation or internal influence. }
\end{aligned}
$$

Equation (1) states that the rate of change in the cumulative number of adopters $(\mathrm{dN}(\mathrm{t}) / \mathrm{dt})$ is proportional to the difference between the market potential $\mathrm{M}$ and the number of previous adopters. The proportionality factor $[\mathrm{p}+\mathrm{q} / \mathrm{M} \mathrm{N}(\mathrm{t})]$ can be interpreted as the probability of adoption at time $t$. This probability is composed of two components: $p$ is interpreted as the probability of spontaneous adoption. The term $[\mathrm{q} / \mathrm{M} \mathrm{N}(\mathrm{t})]$ relates to the probability that adoption will be chosen based on the influence of previous adopters. This probability grows as the number of adopters increases.

To simplify the presentation, Equation (1) can be reoriented in terms of the fraction of the market that is being penetrated rather than the absolute number of adopters. In this case, the market potential can be defined as 1.0. This simplified expression in Equation (2) below now relates to the change in relative cumulative adoptions: 


$$
\mathrm{dF}(\mathrm{t}) / \mathrm{dt}=[\mathrm{p}+\mathrm{qF}(\mathrm{t})][1-\mathrm{F}(\mathrm{t})]
$$

The number of cumulative adoptions at any time, $t$, can be solved by specifying an initial condition that the number of adopters at $t=0$ is 0 . This solution is as follows:

$$
\mathrm{F}(\mathrm{t})=\frac{1-\exp [-(p+q) t]}{1+q / p \exp ([-(p+q)] t]}
$$

The basic diffusion models therefore separate the issue of market penetration rate from market potential. That is why the model in Equation (3) can be compared across technologies - the percentage change in the total penetration does not depend on the size of the market but only on the parameters $\mathrm{p}$ and $\mathrm{q}$.

\section{Estimation Issues}

Issues related to the appropriate estimation procedures for the Bass diffusion model spawned a considerable literature up through the mid-1980s. At least four estimation procedures were proposed by various researchers: 1) ordinary least squares (Bass 1969), 2) maximum likelihood estimators (Schmittlein and Mahajan 1982), 3) nonlinear least squares (Srinivasan and Mason 1986) and Jain and Rao (1989), and 4) algebraic estimation (Mahajan and Sharma 1986).

Mahajan, Mason, and Srinivasan (1986) performed a comparative study of estimation procedures using penetration data for seven products. They concluded that the maximum likelihood and nonlinear least squares procedures provided the best predictions of the four procedures considered. Between those two procedures, nonlinear least squares provided slightly better predictive performance and more valid estimates of the standard errors for the parameter estimates.

As preliminary analysis, the authors looked at three variants of nonlinear least squares model. For the first two variants, the focus is on the number of adopters (X) in each period. Taking the differences of Equation (3) above and including a separate parameter to reflects the total number of adopters $(\mathrm{m})$ results in the following for the first variant:

$$
\mathrm{X}(\mathrm{i})=\underset{1+(\mathrm{q} / \mathrm{p}) \exp \left[-(\mathrm{p}+\mathrm{q}) \mathrm{t}_{\mathrm{i}}\right]}{\left[1-\exp \left(-(\mathrm{p}+\mathrm{q}) \mathrm{t}_{\mathrm{i}}\right]\right.} \quad-\quad \mathrm{m}\left[\frac{1-\exp \left(-(\mathrm{p}+\mathrm{q}) \mathrm{t}_{\mathrm{i}-1}\right]}{1+(\mathrm{q} / \mathrm{p}) \exp \left[-(\mathrm{p}+\mathrm{q}) \mathrm{t}_{\mathrm{i}-1}\right]}+\mathrm{u}_{\mathrm{i}}\right.
$$

Jain and Rao (1989) suggest that that the formulation in (4) gives the ex ante value for X(i) and does not use the ex post information on $\mathrm{X}(1), \mathrm{X}(2), \ldots, \mathrm{X}(\mathrm{I}-1)$. In the Bass model, the probability that an individual who has not purchased the product up to period $\mathrm{t}_{\mathrm{i}-1}$ is given by $\left[\mathrm{F}\left(\mathrm{t}_{\mathrm{i}}\right)-\mathrm{F}\left(\mathrm{t}_{\mathrm{i}-}\right.\right.$ $\left.\left.{ }_{1}\right)\right] /\left(\left(1-\mathrm{F}\left(\mathrm{t}_{\mathrm{i}-1}\right)\right]\right.$. Thus, the number of adopters in the $i$ th time interval is as follows:

$$
\mathrm{X}(\mathrm{i})=\left(\mathrm{m}-\mathrm{N}\left(\mathrm{t}_{\mathrm{i}-1}\right)\right)\left[\mathrm{F}\left(\mathrm{t}_{\mathrm{i}}\right)-\mathrm{F}\left(\mathrm{t}_{\mathrm{i}-1}\right)\right] /\left(\left(1-\mathrm{F}\left(\mathrm{t}_{\mathrm{i}-1}\right)\right]+\mathrm{vi}\right.
$$

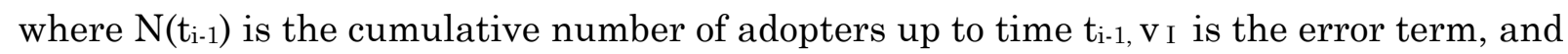
cumulative distribution function is given by Equation (3). This ex post estimation procedure proposed by Jain and Rao uses the actual number of cumulative adoptions in the estimation, as compared to the predicted number in Equation (4). Thus, it is termed the ex post estimation in contrast to the ex ante estimation. 
Mahajan, Mason, and Srinivasan also point out the possibility of estimating the diffusion curve in level rather than differences form (e.g., cumulative sales rather than annual sales). Thus, the cumulative number of adopters is the dependent variable and the specification becomes

$$
\begin{aligned}
& \mathrm{N}(\mathrm{ti})=\mathrm{mF}(\mathrm{t})=\mathrm{m}[\underline{1-\exp [-(\mathrm{p}+\mathrm{q}) \mathrm{t}]}]+\mathrm{wi} \\
& {[1+q / p \exp ([-(p+q)] t]}
\end{aligned}
$$

As Mahajan, Mason, and Srinivasan (1986) indicate, the errors in Equation (6) are likely to be heteroscedastic (i.e., error variance increasing with i) and autocorrelated. Nevertheless, this formulation is somewhat more stable than the differences form and sometimes yields more plausible estimates.

\section{Results}

The results of estimating the Bass diffusion model for ten energy-related technologies are described below. The technologies were placed into four separate categories: 1) lighting, 2) HVAC and refrigeration (HVAC/R), 3) envelope, and 4) other. Table 1 summarizes the technologies for which Bass diffusion models were estimated.

Table 1. Summaries of Technologies Analyzed

\begin{tabular}{|l|c|c|l|}
\hline Technology & Start Year & End Year & \multicolumn{1}{|c|}{ Market Definition } \\
\hline Lighting: & 1986 & 1997 & Corrected power-factor ballasts \\
\hline Electronic ballast & 1986 & 1994 & Incandescent, 15-150 watt \\
\hline Compact fluorescent & 1986 & 1994 & Fluorescent lamps, >30 watt \\
\hline T-8 lamps & 1970 & 1995 & Residential furnaces \\
\hline HVAC and Refrigeration: & 1975 & 1987 & All oil burners \\
\hline Electric Heat Pump & 1982 & 1997 & Gas furnaces \\
\hline Flame retention burner & 1982 & 1995 & No. of supermarkets \\
\hline Condensing gas furnace & 1949 & 1961 & No. of households \\
\hline Advanced Compressor & 1983 & 1996 & Residential windows \\
\hline Room Air Conditioners & 1984 & 1994 & Commercial buildings designed \\
\hline Envelope Technologies: &
\end{tabular}

In most of the cases, the technology was not assumed to ultimately capture all of the market, as defined in the third column of the table. The maximum market potential was judgmentally determined, on the basis of inspection of the data or from other sources.

Table 2 presents the results of the estimation work. The parameter sets labeled in bold are those judged as the most preferred, based on the reasonableness of the estimates and statistical significance. At this point, estimates based on annual adoptions have been used. The annual adoption rates are expressed as a fraction of the total potential market and the maximum fraction of the total market potential is expressed exogenously. The first and third groups of estimates reflect an effort to allow the data to suggest the maximum market potential ( $\mathrm{m}$ rather than $\left.\mathrm{m}^{*}\right)$.

Examining the estimated coefficients indicates that the estimates of the external influence parameter are much more variable than those for the internal influence parameter. One of the lowest values of the internal influence coefficient is found for compact fluorescent lamps, which 
Table 2. Diffusion Curve Parameter Results

\begin{tabular}{|c|c|c|c|c|c|c|c|c|c|c|c|c|}
\hline \multirow[t]{2}{*}{ Product } & \multicolumn{6}{|c|}{ Annual Sales } & \multicolumn{6}{|c|}{ Cumulative Sales } \\
\hline & $\mathbf{p}$ & $\mathbf{q}$ & $\mathbf{m}$ & $\bar{p}$ & $\mathbf{q}$ & $\mathrm{m}^{*}$ & $\mathbf{p}$ & $\mathbf{q}$ & $\mathbf{m}$ & $\bar{p}$ & $\mathbf{q}$ & $\mathbf{m}^{*}$ \\
\hline Electronic ballasts & $\begin{array}{l}.0054 \\
(0.6) \\
\end{array}$ & $\begin{array}{l}.6489 \\
(2.5) \\
\end{array}$ & $\begin{array}{l}.4815 \\
(3.4) \\
\end{array}$ & $\begin{array}{l}.0138 \\
(1.1) \\
\end{array}$ & $\begin{array}{l}.3729 \\
(3.3) \\
\end{array}$ & 0.6 & $\begin{array}{l}.0037 \\
(2.1) \\
\end{array}$ & $\begin{array}{l}.7006 \\
7.3) \\
\end{array}$ & $\begin{array}{l}.4627 \\
(19.5) \\
\end{array}$ & $\begin{array}{l}.0092 \\
(3.3) \\
\end{array}$ & $\begin{array}{l}439 \\
9.3) \\
\end{array}$ & 0.6 \\
\hline $\begin{array}{l}\text { Compact } \\
\text { Fluorescent } \\
\end{array}$ & & & & .0075 & .071 & 0.50 & & & & & & \\
\hline T-8 Lamps & & & & .0041 & .326 & 0.80 & & & & & & \\
\hline Electric Heat Pump & & & & .0118 & 0.459 & 0.23 & $\begin{array}{l}.0054 \\
(1.6) \\
\end{array}$ & $\begin{array}{c}.6228 \\
5.9) \\
\end{array}$ & $\begin{array}{l}.2169 \\
(43.9)\end{array}$ & $\begin{array}{l}.0112 \\
(2.2) \\
\end{array}$ & $\begin{array}{l}.4588 \\
(6.3) \\
\end{array}$ & .23 \\
\hline $\begin{array}{l}\text { Flame retention } \\
\text { burner }\end{array}$ & & & & .0039 & 0.655 & 1.0 & $\begin{array}{l}<.001 \\
(0.3)\end{array}$ & $\begin{array}{l}1.774 \\
(3.7)\end{array}$ & $\begin{array}{l}.8143 \\
(23.9)\end{array}$ & $\begin{array}{l}.0040 \\
(1.1)\end{array}$ & $\begin{array}{l}.655 \\
(4.6)\end{array}$ & 1.0 \\
\hline $\begin{array}{l}\text { Condensing gas } \\
\text { furnace }\end{array}$ & & & & $\begin{array}{l}.070 \\
(1.8) \\
\end{array}$ & $\begin{array}{l}.071 \\
(0.8) \\
\end{array}$ & 0.3 & $\begin{array}{l}.0782 \\
(3.6) \\
\end{array}$ & $\begin{array}{l}.2082 \\
(1.8) \\
\end{array}$ & $\begin{array}{r}.238 \\
(14.7) \\
\end{array}$ & $\begin{array}{l}.0881 \\
(6.1) \\
\end{array}$ & $\begin{array}{l}.0240 \\
(0.6) \\
\end{array}$ & 0.3 \\
\hline $\begin{array}{l}\text { Room air } \\
\text { conditioners }\end{array}$ & & & & .0072 & .423 & 0.33 & & & & & & \\
\hline $\begin{array}{l}\text { Advanced } \\
\text { compressors }\end{array}$ & $\begin{array}{l}.0232 \\
(9.6) \\
\end{array}$ & $\begin{array}{l}.2788 \\
(11.3) \\
\end{array}$ & $\begin{array}{l}.9514 \\
(21.3)\end{array}$ & $\begin{array}{l}0.247 \\
(11.2) \\
\end{array}$ & $\begin{array}{r}.2483 \\
(22.1) \\
\end{array}$ & 1.0 & $\begin{array}{r}.0242 \\
(31.4) \\
\end{array}$ & $\begin{array}{l}.2633 \\
(20.5) \\
\end{array}$ & $\begin{array}{l}.9801 \\
(39.8)\end{array}$ & & & \\
\hline Low-E windows & $\begin{array}{l}.0562 \\
(8.2)\end{array}$ & $\begin{array}{l}.2936 \\
(7.3)\end{array}$ & $\begin{array}{l}.3663 \\
(18.3)\end{array}$ & $\begin{array}{l}.0577 \\
(9.6)\end{array}$ & $\begin{array}{l}.2729 \\
(14.3)\end{array}$ & 0.37 & & & & $\begin{array}{l}.0565 \\
(25.0)\end{array}$ & $\begin{array}{l}.2819 \\
(27.3)\end{array}$ & 0.37 \\
\hline $\begin{array}{l}\text { DOE-2 Building } \\
\text { Model }\end{array}$ & $\begin{array}{c}.00001 \\
(0.5) \\
\end{array}$ & $\begin{array}{l}1.18 \\
(4.8) \\
\end{array}$ & $\begin{array}{l}.279 \\
(6.4) \\
\end{array}$ & .0005 & .656 & .50 & & & & & & \\
\hline
\end{tabular}

reflect their extremely slow penetration into the market to date. (In addition to their high initial price, Haddad [1994] suggests that industrial organization, retail incentives, and social convention are additional reasons for the atypically slow adoption of this technology.) On the other extreme is the flame retention oil burner, whose adoption was accelerated by the increase in oil prices during the Iranian revolution in the late 1970s. In spite of these extremes, the simple average internal influence coefficient of 0.38 is the same as the average for 213 technologies as reported by Sultan, Farley, and Lehmann (1990). In that study, the average external influence was 0.03 , compared with an average 0.018 for the ten energy-related technologies.

\section{References}

Bass, F.M. 1969. "A New Product Growth Model for Consumer Durables" Management, Vol. 15 (January 1969), pp. 215-227.

Electric Power Research Institute (EPRI). 1991. Market Penetration of New Technology, Programs and Sciences, EPRI CU-7011, February 1991.

Haddad, B. M. 1994. "Why Compact Fluorescent Lamps Are Not Ubiquitous: Industrial Organization, Incentives, and Social Convention", in Proceeding of the ACEEE 1994 Summer Study on Energy Efficiency in Buildings, Vol. 10, pp. 77-84. American Council for an EnergyEfficient Economy, Washington D.C. 
Mahajan, V., C. Mason, and V. Srinivisan. 1986. "An Evaluation of Estimation Procedures for New Product Diffusion Models." In Innovation Diffusion Models of New Product Acceptance, V. Mahajan and Y. Wind, eds., Cambridge, MA: Ballanger.

Mahajan, V. and S. Sharma. December 1986. "A Simple Algebraic Estimation Procedure for Innovation Diffusion Models," Technological Forecasting and Social Change. 30:331-45.

Schmittlein, D.C. and V. Mahajan. 1982. "Maximum Likelihood Estimation for an Innovation Diffusion Model of New Product Acceptance," Marketing Science. 1(Winter): 57-78.

Sultan, F., J.U. Farley, and D.R. Lehmann. 1990. "A Meta-Analysis of Applications of Diffusion Models," Journal of Marketing Research, Vol. XXVII (February 1990), pp. 70-77.

Srinivzsan, V. and C. Mason 1986. "Nonlinear Least Squares Estimation of New Product Diffusion Models,” Marketing Science. 5 (Spring):169-78.

\section{Bibliography}

Gilshannon, S.T. and D.R. Brown. 1996. Review of Methods for Forecasting the Market Penetration of New Technologies, PNNL-11428, Pacific Northwest National Laboratory.

Kamakuru, W.A. and S.K. Balasubramanian. 1988. "Long-Term View of the Diffusion of Durables," International Journal of Research in Marketing, Vol. 5, pp. 1-13.

Mahajan, V., E. Muller, and F. Bass. January 1990. "New Product Diffusion Models in Marketing: A Review and Directions for Research," Journal of Marketing, Vol. 54:1-26.

Roberts, G.F. and D.L. Greene. January/February 1983. "A Method for Assessing the Market Potential of New Energy-Saving Technologies," In IEEE Transactions on Systems, Man, and Cybernetics, Vol. SMC-13, No. 1.

Stoneman, P. 1987. The Economic Analysis Technology Policy. Oxford: Clarendon Press.

Teotia, A.P.S. and P.S. Raju. December 1986. "Forecasting the Market Penetration of New Technologies Using a Combination of Economic Cost and Diffusion Models," Journal of Product Innovation Management, 3:225-237.

Thomas, R.J. 1985. "Estimating Market Growth for New Products: An Analogical Diffusion Model Approach." Journal of Product Innovation Management, Vol. 2, pp. 45-55.

\section{List of Terms}

BTS Office of Building Technology, State and Community Programs

DOE U.S. Department of Energy

EPRI Electric Power Research Institute

PNNL Pacific Northwest National Laboratory 


\section{Appendix D Methodology}




\section{Appendix D \\ GPRA Metrics Methodology}

This appendix describes the calculation methodology used within the Building Energy Savings Estimation Tool (BESET) and the National Energy Modeling System (NEMS) to estimate the energy savings for programs in the following decision units:

- Community Energy Program

- Energy Star Program

- Residential Buildings Integration

- Commercial Buildings Integration

- Equipment, Materials, and Tools.

The programs in the other three decision units, State Energy Program, Weatherization Assistance Program, and Technology Roadmaps and Competitive R\&D, were calculated within spreadsheets using the inputs outlined in the main report for these appendixes - Documentation for FY 2003 BTS GPRA Metrics.

Because of the number and length of the tables in this appendix, all the tables are shown at the end of the discussion.

\section{Methodology for Whole-Building Programs}

This section addresses programs that target the building envelope, a whole-building design approach, or the total building system and that are modeled as improvements to the building envelope. Envelope programs are characterized by a reduction in space conditioning and water heating load from changes in the building system or envelope. The following programs in the U.S. Department of Energy's (DOE's) Office of Building Technology, State and Community Programs (BTS) are characterized by the whole-building approach:

- Rebuild America (in Community Energy Program decision unit)

- Residential Buildings R\&D (in Residential Buildings Integration decision unit)

- Commercial Buildings R\&D (in Commercial Buildings Integration decision unit)

- Analysis Tools and Design Strategies (in Equipment, Materials, and Tools decision unit).

This section uses the FY 2001 Residential Buildings R\&D program to illustrate the calculation methodology. The steps below are involved in calculating the energy savings associated with the Residential Buildings R\&D program and are discussed in the next subsections:

- Determine the size of the potential market.

- Determine the number of units affected by the BTS program.

- Determine the base space conditioning and water heating end-use loads.

- Determine the space conditioning and water heating end-use loads after program implementation.

- Calculate the energy savings. 


\section{Determine Size of the Potential Market}

The Residential Building R\&D program targets new residential construction. The size of this potential market is shown in Table D.1 and is part of the baseline assumptions (see Appendix A). Table D.1 shows the new residential building stock, in millions of households, for the north and south regions. The stock of new buildings is defined as those built within the year, not the total new building stock (all buildings built since 2001). The last row of the table represents the cumulative new building stock total (all buildings built since 2001).

\section{Determine Number of Units Affected by the BTS Program}

The number of units affected by the BTS program is calculated using the program penetration rates (Table D.2) and the building stock (Table D.1). The penetration rates are applied to the appropriate market segment to compute the number units impacted by the BTS program (see Table D.3). For the new building stock, this figure represents the number of impacted housing units within that year, not the cumulative number. Because energy savings are accumulated over time - a housing unit impacted by the BTS program in 2001 will continue to save energy throughout its life - the number of impacted units must be summed to represent the total number of impacted housing units in the given year (see Table D.4). Therefore, the total number of impacted units listed for single-family houses for 2005 in Table D.3 (.016) represents the number built between 2004 and 2005 that are impacted by the BTS program, while the corresponding number in Table D.4 (.040) represents the number of units impacted since the program was implemented or 2001, whichever is later.

Some envelope programs affect the existing building stock, unlike the Residential Building R\&D program. The existing building stock is defined as the total stock in 2001 that survive in each subsequent year. The penetration into that building stock therefore represents the cumulative number of units to that time period, negating the need to accumulate the number of installed units.

\section{Determine Base Space Conditioning and Water Heating End-Use Loads}

The end-use loads for heating, cooling, and water heating for new residential buildings are shown in Table D.5 and are part of the baseline assumptions (see Appendix A). The performance improvements for envelope programs are characterized by reductions in the space conditioning and water heating loads. Therefore, the base energy consumption does not have to be explicitly calculated. Instead, the load reduction is applied to the base load to determine the new load; and the resulting difference in loads is used to calculate energy savings.

\section{Determine Space Conditioning and Water Heating End-Use Loads After Program Implementation}

The performance inputs for envelope programs are defined in terms of percent load reductions. Table D.6 presents the percent load reductions for the Residential Building R\&D program.

The load reductions are applied to the corresponding end-use load segment to determine the building-level load reductions by end use, presented in Table D.7. The building-level load reductions are translated into aggregate load reductions by region by multiplying the 
calculated building-level load reductions (in absolute terms, KBtu/sq ft or MMBtu/ household) by cumulative installed units for that region (given in Table D.4). Tables D.8 and D.9 present the regional load reductions by end-use.

\section{Calculate Energy Savings}

The regional load reductions must be translated into regional energy savings. To do this, baseline assumptions regarding existing equipment efficiencies and existing equipment market shares are used. First, the regional load reductions are divided by the baseline existing equipment efficiencies (listed in Table D.10), which yields potential energy savings by equipment type and end use, as shown in Table D.11.

The potential energy savings assume that each equipment type has $100 \%$ of the market, so the actual equipment market shares must then be applied. Table D.12 shows the existing baseline equipment market shares. The market share for each equipment type is multiplied by the potential energy savings calculated in Table D.11 to determine the actual energy savings, as shown in Table D.13.

The savings calculated in Table D.13 are aggregated by fuel type to determine the total delivered electric savings, total primary electric savings (equal to total delivered electricity multiplied by electricity conversion factor that varies by year), total natural gas savings, total oil savings, and total primary savings. Table D.14 presents the energy savings.

\section{Equipment and Standards - General Methodology Using the National Energy Modeling System}

Most of the programs in the Equipment, Materials, and Tools decision unit target specific types of equipment within a building or standards directed toward the usage of specific equipment. Equipment programs are characterized by new equipment efficiencies and are compared with "baseline" efficiencies to calculate energy savings. To determine the penetration of the BTS equipment relative to the more conventional equipment, the National Energy Modeling System (NEMS) for the Energy Information Administration's (EIA's ) Annual Energy Outlook (AEO 2000) has been used as part of the FY 2002 GPRA metrics to estimate the energy savings of various BTS equipment programs.

The NEMS commercial and residential demand modules generate forecasts of energy demand (energy consumption) for the commercial and residential sectors. The commercial demand module generates fuel consumption forecasts for electricity, natural gas, and distillate fuel oil. These forecasts are based on energy prices and macroeconomic variables from the NEMS system, combined with external data sources. The residential model uses energy prices and macroeconomic indicators to generate energy consumption by fuel type and census division in the residential sector. The commercial and residential demand modules are described in the following subsections.

\section{Commercial Demand}

Appendix D - 3 
This module includes assembly, education, food service, food sales, health care, lodging, mercantile/service, and office buildings. Commercial energy demand within NEMS is calculated in four basic steps. The first step is to forecast commercial sector floorspace. The second step is to forecast energy services such as space conditioning equipment, lighting, water heating, and refrigeration. The third step is to select specific technologies to meet the demand of energy services. This step involves modeling consumer behavior and capturing the decision between such equipment as incandescent lights and fluorescent lights. The final step involves determining how much energy will be consumed by the equipment chosen to meet the demand for energy services.

This third step is a key element in calculating the estimated energy savings of a given technology promoted by a particular BTS program. Within this step, consumers are assumed to purchase energy-using equipment to meet three types of service demands: services for new buildings, replacement of old equipment that is at the end of its technical life, and replacement of old equipment that is at the end of its economic life (although it still may be technically viable). The NEMS commercial model is coded to allow the use of several possible assumptions about consumer behavior to model this decision process. The assumptions are designed to represent empirically the range of economic factors that most influence the consumer's decision and include the following:

1. Consumer buys the equipment with the minimum life-cycle cost.

2. Consumer buys equipment that uses the same fuel as existing and retiring equipment but minimizes costs across technologies using that fuel.

3. Consumer buys (or keeps) the same technology as the existing and retiring equipment but chooses among different efficiency levels based on minimum life-cycle cost.

The model is designed to choose among a discrete set of technologies that are exogenously characterized by commercial availability, capital costs, operating and maintenance costs, efficiencies, and lifetime. For GPRA metrics, the menu of equipment may be altered to include relevant BTS program equipment, technological innovations, and standards. The NEMS design can accommodate a changing menu of technology choices, recognizing that changes in energy prices and consumer demand may significantly change the set of relevant technologies that the model user wishes to consider.

\section{Residential Demand}

The residential sector demand module includes single-family, multifamily, and mobile home dwellings. Residential energy demand is modeled using a sequence of five steps. The first step is to forecast housing stock. The second step is to select the specific technologies to meet the demand for each energy service (e.g. furnaces and heat pumps). The third step is to forecast appliance stocks that are required by each end-use service. The fourth step is to forecast changes in building-shell integrity. Building-shell efficiency in new construction is assumed to improve over the forecast period because of stricter building codes and other efficiency programs and may fluctuate in response to fuel price changes from the base year. The final step is to calculate the energy consumed by the equipment chosen to meet the demand for energy services.

As with the commercial model, the GPRA metrics methodology involves modifying the technology performance and cost inputs to reflect the BTS-developed equipment. The technology and equipment selection simulates the behavior of residential consumers based on the relative importance of life-cycle costs, capital costs, and operating costs of competing technologies within 
a service. Decisions on new and replacement equipment reflect additional factors beyond the traditional life-cycle cost methodology, including space heating fuel choice and previous equipment choices. The technology and equipment selection allocates end-use services based on a defined equipment menu of the various technologies and fuels that compete in the market.

\section{Methodology for Specific Energy Star Programs}

This section covers the methodological approach to calculating energy savings for the programs related to the following types of equipment:

- water heaters (Energy Star gas and electric water heaters)

- refrigerators (Energy Star refrigerators)

- clothes washers (Energy Star clothes washers)

- air conditioners (Energy Star room air conditioners)

- dishwashers (Energy Star dishwashers).

The modifications to the NEMS input file (RTEKTY) for each appliance with an Energy Star program are described below. The baseline assumptions made by EIA, the changes in the Beta1 coefficients, and the resulting changes in the market shares for the most energy-efficient products are documented. For a few appliances, some changes were made in the baseline assumptions made by EIA; the reasons for these changes are briefly discussed.

\section{Data Input Modifications for Energy Star Refrigerators}

Baseline Modifications. EIA uses four separate models to represent the range of energy efficiencies in the refrigerator market. The first three models are conventional top-mount freezer models with a total capacity of 18 cubic feet. The fourth model is a through-the-door model (for water and ice) and does not compete with the first three models. The market share of the through-the-door model is a constant $27 \%$ over the forecast horizon. A review of Arthur D. Little's (ADL's) (1998) efficiency and cost forecasts, as well as a recent paper from Oak Ridge National Laboratory (ORNL) (Vineyard and Sand 1998), suggests some changes to EIA's assumptions used in the $A E O 2000$ projection are warranted.

As part of the EIA forecast, an assumption is made that the 2001 standard (Model 1) yields no increase in cost. Table D.15 shows the EIA efficiency and cost assumptions. This assumption appears to contradict some of the ADL findings. The ADL performance/cost characteristics information suggests that a $460-\mathrm{kWh} / \mathrm{yr}$ unit would have an installed cost of $\$ 580$ to $\$ 700$. To be conservative, an installation cost of $\$ 600$ could be assumed. Because a $478-\mathrm{kWh} / \mathrm{yr}$ unit is nearly as efficient as the $460-\mathrm{kWh} / \mathrm{yr}$ unit, one would expect it would be only negligibly less expensive. Using this logic, the cost of the $478-\mathrm{kWh} / \mathrm{yr}$ unit is assumed to be about $\$ 580$. These revised assumptions are included in Table D.16.

The ADL report suggests that a 460-kWh/yr model represents a typical model after 2002. A highefficiency model is specified to consume $400 \mathrm{kWh}$ per year. However, this specification is for a 20 -cubic-foot model rather than 18 cubic feet. ADL suggests a cost differential of $\$ 100$ to $\$ 120$ between these two models.

The 1998 paper by ORNL's Vineyard and Sand (1998) adds some support to this revision in the cost structure. Vineyard and Sand start with a "1996 model baseline unit" of 20 cubic feet that 
uses $613 \mathrm{kWh} /$ year. The baseline is already 16\% more efficient than the 1993 standard (2.01 $\mathrm{kWh}$ /day) resulting from the National Appliance Energy Conservation Act. From this baseline, Vineyard and Sand focus on two high-efficiency designs. The most aggressive design would reduce energy by $273 \mathrm{kWh} / \mathrm{yr}$ at a retail cost increase of about $\$ 270$. A more cost-effective unit would consume $1.16 \mathrm{kWh} /$ day $(423 \mathrm{kWh} / \mathrm{yr}$ ) at a projected cost increase of $\$ 106$.

Given this information, the resulting estimated cost increase of $\$ 100$ between the 460 - and 400 $\mathrm{kWh}$ /day units appears to be more reasonable (see Table D.16) than EIA's incremental cost of $\$ 150$. The ORNL baseline unit is less efficient than the 2001 standard and achieves a 30\% energy reduction with a little more than a $\$ 100$ cost increase. This suggests that the $13 \%$ efficiency improvement (460 to 400) between models 2 and 3 could be achieved for $\$ 100$ or less.

Modeling. The two parameters are labeled by EIA as Beta1 and Beta2. Beta1 is used as multiplicative factor with the initial cost of the appliance. Beta2 is used to multiply the annual energy cost. The sum of the two products (i.e., Beta 1 * initial cost + Beta $2 *$ operating cost) is used in the logit specification to yield market shares for each technology. The Beta1 and Beta2 coefficients are contained with the cost and efficiency data inputs in the file RTEKTY.

As a rough approximation, the ratio of Beta1/Beta2 can be interpreted as the consumer discount rate for the specific appliance. In the residential NEMS module, the Beta1 and Beta2 coefficients vary among appliances, as do the resulting discount rates. For example, the implied discount rates for refrigerators are $16 \%$. On the other hand, the discount rate is estimated to be over $80 \%$ for electric water heaters.

The EIA used the parameters -0.0229 (Beta1) and -0.1207 (Beta2) to reflect the discount rate used by consumers in evaluating various refrigerators are. This translates into approximately a 19\% discount rate (Beta1/Beta2).

For modeling purposes, the focus of the Energy Star program is assumed to increase the market share of the $400-\mathrm{kWh} / \mathrm{yr}$ refrigerator. This unit is about $16 \%$ more efficient than a unit meeting the most recent national standard. To generate a larger market share for this model, the Beta1 coefficient was changed to -0.0055 .

The NEMS residential model does not automatically produce an output table that displays the shares of individual appliances with different efficiencies. These shares are computed within the model for each building type (single family, multifamily, and mobile home) and by the nine census divisions. The NEMS-BTS model was modified to display the shares for the single-family market segment for three separate years: 2005, 2010, and 2020. The results from NEMS for 2005 and 2010 are shown in Table D.17.

\section{Energy Star Clothes Washers}

Modeling the energy savings clothes washers is complex because energy savings can be achieved by reducing the consumption of the motor, reducing hot water use, or reducing dryer use. The most efficient new technology is the horizontal-axis design, which achieves the bulk of its energy savings by the reduction in hot water use. 
The residential NEMS input file (RTEKTY) includes a column of factors that relates to hot water. The (unit less) factors can be used to adjust the hot water load associated with clothes washers and dishwashers. In preliminary model runs, the values associated with clothes washers appeared to be too low compared with the information supplied by Lawrence Berkeley National Laboratory (LBNL) in support of an efficiency standard for clothes washers. These factors were adjusted from 0.67 to 2.00 for vertical-axis machines. The coefficient for the horizontal-axis machine was increased from 0.24 to 0.40 . The value for the vertical axis machine was estimated by making runs of the model with and without any hot water and observing the resulting energy consumption. The LBNL analysis suggests that $80 \%$ to $90 \%$ of the energy consumption of clothes washers is attributable to water heating. Table D.18 shows the original and revised NEMS inputs for clothes washers.

For the Energy Star program, the discount rate was adjusted to achieve larger shares for the more efficient vertical axis machine as well as the horizontal axis machine. In the baseline run, horizontal axis machines had zero market shares (thus, the model is not consistent with current sales information that shows that these machines are being sold in limited numbers.) The goal of the GPRA exercise was to increase the market share of these machines to $8 \%$ to $10 \%$ of the market. This result was roughly achieved by reducing the Beta1 coefficient in the model from 0.03811 to 0.0101 . Table D.19 shows the results of the NEMS model runs for clothes washers.

\section{Energy Star Hot Water Heaters}

Separate sets of NEMS runs were made for electric water heaters and gas water heaters to model the effects of Energy Star programs.

Electric Water Heater. The key NEMS used by EIA for the AEO 2000 are shown in Table D.20. With these assumed costs, the model projects a zero share for heat pump water heaters.

The Energy Star program was assumed to target high-efficiency electric water heaters whose efficiencies exceed 0.9. As shown in Table D.20, two such units are shown, with efficiencies of 0.95 and 0.96. By 2005, the installed cost of the high-efficiency unit (at the 0.96 efficiency level) is assumed to fall to $\$ 475$.

The logit parameters in the NEMS model related to the choice of electric water heaters are 0.01619 (Beta1) and -0.01952 (Beta2), implying a discount rate of about 83\%. For the GPRA estimate for the Energy Star program, the Beta1 coefficient was reduced to 0.0082, implying a discount rate of about $43 \%$. This change resulted in an increase in the market shares of the (assumed) Energy Star products of roughly 11 percentage points. The specific results are shown in Table D.21.

Gas Water Heaters. The key NEMS inputs used by EIA for the AEO 2000 are shown in Table D.22. With these assumed costs, the model projects a zero share for the (near) condensing units that have efficiencies greater than 0.8 .

The Energy Star program was assumed to target high-efficiency gas water heaters whose efficiencies are 0.6 or higher. As shown in Table D.20, two such units are shown, with efficiencies of 0.6 and 0.63. By 2005, the installed cost of the high-efficiency unit (at the 0.60 efficiency level) is assumed to fall from $\$ 400$ to $\$ 375$. 
The logit parameters in the NEMS model related to the choice of gas water heaters are -0.05393 (Beta1) and -0.1136 (Beta2), implying a discount rate of about 47\%. For the GPRA estimate for the Energy Star program, the Beta1 coefficient was reduced to 0.0323, implying a discount rate of about 28\%. This change resulted in an increase in the market shares of the (assumed) Energy Star products of a little more than 12 percentage points. The specific results are shown in Table D.23.

\section{Energy Star Room Air Conditioners}

For the year 2005, EIA assumes that efficiencies of room air conditioners will range from a low of 2.83 SEER (seasonal energy efficiency ratio) to a high of 3.52 SEER. In the AEO 2000 input file for the residential NEMS module, two models were at the low end of this range (SEER $=2.83$, SEER = 2.93), while two models were at the high end of the range. To achieve a more realistic set of choices, a model with an intermediate efficiency of 3.11 was added and the unit at the 2.93 (SEER) level was dropped. The increase in cost to go from a SEER of 2.83 to 2.93 was assumed to be $\$ 30$. Table D.24 shows both the original NEMS input data and the revised data.

The high-efficiency units with an energy-efficiency ratio (EER) of $>3.4$ were assumed to fall under the Energy Star program. In the base case, the combined market share for the units with SEERs of 3.43 and 3.52 were less than 1\%. The split between the lowest efficiency unit (SEER $=2.83$ ) and the intermediate efficiency unit (SEER $=3.11)$ was generally about $75 \% / 25 \%$ in favor of the lowest efficiency model.

The logit parameters in the NEMS model related to the choice of room air conditioners are -0.017 (Beta1) and -0.12 (Beta2), implying a discount rate of over 100\%. For the GPRA estimate for the Energy Star program, the Beta1 coefficient was reduced to 0.007, implying a discount rate of about 58\%. This change resulted in an increase in the market shares of the (assumed) Energy Star products of a little more than 12 percentage points. (In addition, the market share of the model with the intermediate efficiency of 3.11 increased from approximately $25 \%$ to $35 \%$, also contributing to estimated energy savings). Table D.25 shows the specific results for the highefficiency model.

\section{Energy Star Dishwashers}

The NEMS baseline (AEO 2000) data input for the year 2005 shows three dishwashers, with energy factors $0.46,0.59$, and 0.71. The associated costs of these units are shown in Table D.26. Given the cost structure and logit choice parameters, the model suggests that consumers select slightly more than $6 \%$ of dishwashers with the 0.59 energy factor and virtually none of the very high efficiency units.

The logit parameters in the NEMS model related to the choice of dishwashers are -0.02738 (Beta1) and -0.02413 (Beta2), implying a discount rate of over 100\%. For the GPRA estimate for the Energy Star program, the Beta1 coefficient was reduced to 0.01338, implying a discount rate of about 55\%. This change resulted in an increase in the market share of the intermediate efficiency unit (energy factor $=0.59$ ) of about 15 percentage points (to over $20 \%$ ). The market share of the very high efficiency unit increases to a little more than $3 \%$. The specific results for the two high-efficiency models are shown in Table D.27.

\section{NEMS Modeling of Emerging Technologies}

Appendix D - 8 
NEMS was used to estimate the energy savings associated with the products being developed under BTS's Emerging Technologies program. The NEMS residential model used was modified to represent two technologies under this program: 1) heat pump water heater and 2) condensing gas water heater. The high-efficiency rooftop air conditioner program was modeled in the NEMS commercial model.

The modifications to the NEMS input files (RTEKTY.txt for residential, KTECH.wk1 for commercial) for each type of equipment in the Emerging Technologies program are described below. The baseline assumptions made by EIA, the cost and performance attributes of the BTSsponsored technologies, and the resulting market shares for these most energy-efficient products are documented. For a few appliances, some changes were made in EIA's baseline assumptions; the reasons for these changes are briefly discussed.

\section{General Methodology}

For the FY 2002 GPRA effort, the water heater technologies funded by the Emerging Technologies program were modeled only in the residential model. The residential model uses a logit specification to estimate the market shares of specific technologies for a given type of

appliance. For each appliance, two parameters generally influence how consumers trade off the initial purchase cost versus the annual operating cost of the appliance. The annual operating cost of course depends on the energy efficiency of each technology (or "model") and the price of energy.

\section{Data Input Modifications for Specific Appliance and Emerging Technology Programs}

The modifications to the NEMS input file (RTEKTY) for each appliance being developed under the Appliance and Emerging Technologies program are described below. The baseline assumptions made by EIA, the performance and cost assumptions for the BTS-sponsored technologies, and the resulting changes in the market shares for the most energy-efficient products are documented. For a few appliances, some changes were made in EIA's baseline assumptions; the reasons for these changes are briefly discussed.

Heat Pump Water Heater. The input file used for the AEO 2000 includes several categories of heat pump water heaters, two of which are shown in Table D.28. Inexplicably, the lower-cost unit is assumed to have a higher efficiency. With the discount rates used in the $A E O 2000$ for electric water heaters, only a very small number of the $\$ 1,000$ unit are predicted to be sold (and none of the higher-cost unit). A more moderately priced heat pump unit is assumed to become available in 2005 , with a cost of $\$ 900$ and an energy factor of 2.0. By 2015, the cost falls to $\$ 800$ and the energy factor increases to 2.2 .

The original AEO 2000 input file does not reflect the pending water heater standards that are scheduled to take effect in 2004. Two modifications were made to crudely account for these standards: 1) technology 1 was assumed to be unavailable after 2003, and 2) the efficiency for technology 2 was changed to 0.89 with an unchanged cost. The new standard calls for a 50-gallon unit (as assumed in the table) to have an energy factor between 0.89 and 0.90 . These modifications are shown at the top of Table D.28.

The Appliance and Emerging Technologies program is assumed to lead to a more rapid commercialization of a moderately priced heat pump water heater, first available in 2004. But the principal impact of the program is to achieve a lower cost than the unit assumed to be 
introduced in 2005 in the AEO base case. As shown in Table D.28, the units are assumed initially to have energy efficiency rating of 2.0 and a cost of $\$ 700$ (installed). By 2010, further development will yield a unit with slightly higher efficiency (2.10) at a lower cost $(\$ 650)$. By 2015 , further improvements lead to an efficiency of 2.2 at a cost of $\$ 600$. (Note that the $A E O$ 2000 input assumptions already include a heat pump water heater but at a substantially higher cost). (For modeling, the units introduced in the Emerging Technologies program replace the $\$ 900$ and $\$ 800$ units that are part of the AEO base case, and therefore the base case units are not shown in Table D.28).

One issue related to assessing benefits of this technology with the NEMS model is the appropriate discount rate to use. The logit parameters in the NEMS model related to the choice of electric water heaters are -0.01619 (Beta1) and -0.01952 (Beta2), implying a discount rate of about $83 \%$. At this discount rate, the high initial cost of the heat pump water heater, even with its much higher efficiency, discourages most consumers from choosing this technology. The NEMS results shown in Column 2 of Table D.29 show that the market share does not quite reach $5 \%$, even with the third-generation unit assumed in the analysis. (Note: The market shares in this discussion pertain only to electric water heaters.)

A more realistic overall assessment of the program is obtained by assuming that the ongoing Energy Star program for water heaters provides impetus for increased market acceptance of the heat pump water heater. In this scenario, the changes in the discount rates assumed for Energy Star program are combined with the introduction of the (lower-cost) heat pump water heater. As for the GPRA estimate for the Energy Star program, the Beta1 coefficient in the NEMS model was reduced to 0.0072 , implying a discount rate of about $37 \%$. As shown in Table D.29, the lower discount rates generate much higher penetrations of the heat pump water heater, ultimately reaching nearly $25 \%$ of sales by 2010 . (While Table D.29 displays the shares for only new homes, the shares for the replacement market are similar).

The program's energy savings (not shown here) are thus calculated as the difference between NEMS model runs that 1) include the heat pump waters assumed in the AEO base case and 2) substitute the lower-cost units assumed to stem from the Emerging Technologies program. In both runs, the adjustments to the discount rate (via the Beta1 coefficient) are the same as those used in evaluating the Energy Star program for water heaters. The Energy Star program is assumed to promote greater adoption of both conventional resistance units with energy factors of 0.95 or higher as well as heat pump water heaters. In essence, the program's savings are calculated as the difference between an Energy Star program with and without the units developed under the Emerging Technologies program. (The program summary contains further discussion of the savings from the Energy Star program without these lower-cost heat pumps.) Under Energy Star the market share of the $\$ 900$ heat pump unit in 2010 is 0.046 compared with a 0.239 share of the $\$ 700$ unit, as shown in Table D.29.)

Finally, the assumption of an ongoing Energy Star program raises the question of whether that program should receive some of the credit for energy savings brought about by this technology. No clear methodology exists for decomposing the benefits between applied R\&D program and market conditioning activities. Clearly, without the existence of the more efficient technology, Energy Star is limited in the impact that it can make toward reducing actual energy use. If such an attribution must be made for the GPRA process, $70 \%$ of the savings are proposed to be assigned to the Emerging Technologies program and 30\% to Energy Star. 
Condensing Gas Water Heater. The original AEO 2000 input file does not reflect the pending water heater standards that are scheduled to take effect in 2004. To account for these standards in the gas water heater market, the technologies with energy factors $<0.60$ (0.54 and 0.58) were specified to be unavailable after 2003. These modifications are shown in the top two lines of Table D.30.

EIA includes a high-efficiency condensing gas water heater in its menu of technology choices for the AEO 2000. As Table D.30 shows, these units have very high costs. Not surprisingly, the model yields negligible market shares for this technology.

The Emerging Technologies program is assumed to lead to the commercialization of a moderately priced condensing gas water heater, first available in 2003. As Table D.30 shows, the units are assumed initially to have an energy efficiency rating of 0.8 and cost $\$ 550$ (installed). By 2010 , further development is assumed to yield a unit with slightly lower cost (\$525).

As with the heat pump water heater, an issue related to the assessment of benefits with the NEMS model is the appropriate discount rate to employ. The logit parameters in the NEMS model related to the choice of gas water heaters are -0.05393 (Beta1) and -0.1136 (Beta2), implying a discount rate of about $47 \%$. At this discount rate, the higher initial cost of the BTSsponsored condensing gas water heater, even with its much higher efficiency, discourages most consumers from choosing this technology. The NEMS results shown in Column 2 of Table D.31 show that the market share only reaches about $1 \%$, even with the lower-cost second-generation unit assumed in the analysis.

As with the heat pump water heater, a more realistic overall assessment of the program is obtained by assuming that the ongoing Energy Star program for water heaters provides impetus for increased market acceptance of the condensing gas water heater. In this scenario, the changes in the discount rates assumed for Energy Star program are combined with the introduction of the (lower-cost) condensing gas water heater. As for the GPRA estimate for the Energy Star program, the Beta1 coefficient in the NEMS model was reduced to -0.03593, implying a discount rate of about $32 \%$. As shown in Table D.31, the lower discount rates generate much higher penetrations of the condensing water heater, ultimately reaching nearly $10 \%$ of sales by 2010 . The inputs in the AEO 2001 assume the introduction of a high efficiency noncondensing unit in 2015. This assumption is the principal explanation for why the share of the condensing unit drops between 2010 and 2020. (While Table D.31 displays the shares for only new homes, the shares for the replacement market are similar).

Again, the assumption of an ongoing Energy Star program raises the question of whether that program should receive some of the credit for energy savings brought about by this technology. No clear methodology exists for separating the benefits between applied R\&D program and market conditioning activities. Without the more efficient technology, Energy Star is limited in the impact it can make toward reducing actual energy use. If such an attribution must be made for the GPRA process, 70\% of the savings again is proposed to be assigned to the Emerging Technologies program and 30\% to Energy Star.

High-Efficiency Rooftop Air Conditioner. The rooftop air conditioner program uses competitive procurements of large numbers of units to stimulate the production of high-efficiency equipment. Its immediate goal is to get high-efficiency equipment installed in buildings owned by the federal government other state and local agencies. In the long term, however, a key outcome of the program is to provide incentives for manufacturers to reduce the cost of this equipment to all potential and private sector buyers. 
With this long-term goal in mind, the assumed costs of high efficiency roof top air conditioners were adjusted in the NEMS commercial model to reflect the principal influence of this program. In NEMS, two air conditioners were specified in the rooftop category - a baseline unit $(\mathrm{EER}=8.5)$ and a high-efficiency unit $(\mathrm{EER}=11.6)$. No subgroups were distinguished by capacity (e.g., 65 to $135 \mathrm{kBtu} / \mathrm{h}$ vs. 135 to $240 \mathrm{kBtu} / \mathrm{h})$.

An alternative technology spreadsheet was developed to model the rooftop initiative in the Emerging Technologies. (In most recent version of NEMS, the technology cost and performance inputs are in a spreadsheet). In this spreadsheet, the user can adjust the incremental cost between baseline unit and the high-efficiency unit.

For the GPRA analysis, the incremental cost was reduced by $40 \%$. Given the proportion of the market assumed in the NEMS to display high discount rates in the selection of equipment, this cost reduction was necessary to yield a 9\% penetration of the high-efficiency unit in 2005 . The penetration rate falls to $6 \%$ in 2010 possibly the result of a couple of factors: 1) greater efficiency of the baseline unit or 2) lower energy costs. As this point, the exact reasons for this behavior have not yet been investigated. By 2020, the proportion of the total stock using the highefficiency unit is about $5 \%$.

\section{GPRA Envelope Calculations Using NEMS}

The general approach for GPRA envelope calculations using NEMS was to simulate the effect of an envelope technology using the Facility Energy Decision System (FEDS) ${ }^{1}$ model for many different building types, sizes, vintages, and locations. The heating and cooling loads were calculated for each building with and without the envelope technology being evaluated. The changes in the heating and cooling loads were then used to modify the heating and cooling envelope factors used in NEMS. These factors were input as a vector for each building type and census region; these vectors captured both the thermal impact and the expected market penetration by year. Market penetration estimates were based on input from the DOE Program Manager or their representatives.

\section{FEDS Modeling}

To estimate the national impact of introducing a new envelope technology, the impact of that technology must be accurately captured within the buildings where it is likely to be employed. For each technology, the impact was simulated in 3,960 commercial buildings and 1,188 residential buildings representing all combinations of building type, size, vintage, and location (see Table D.32).

\section{Aggregating FEDS Results for NEMS}

Because NEMS only models one of each building type in each of the nine census regions, the FEDS results needed to be aggregated for input into NEMS.

City Weights. The cities shown in Table D.32 were selected for the FEDS analysis because the weather is characteristic of the climate in the different portions of the census regions. Because

\footnotetext{
1 Pacific Northwest National Laboratory (1998).
} 
NEMS operates on a census region bases, weighted averages of the FEDS results for individual weather cities were produced to represent the loads within a census region. Table D.33 shows the weights given to each city for each census region.

Floor Area Weights. The fraction of floor space within each size category for each commercial building type was determined using data from Commercial Buildings Energy Consumption Survey (CBECS) ${ }^{2}$ and is shown in Table D.34.

Table D.35 shows the fraction of floor space within each size category for each residential building type (single family, mobile homes, and multifamily). The data for single-family and mobile homes was determined using data from Residential Energy Consumption Survey (RECS) ${ }^{3}, 4$ and the data for multifamily homes was determined using data from RECS and apartment stock data from the National Multi-Housing Council. ${ }^{5}$

Vintage Weights. All vintages were given equal weighting.

Market Penetration. The DOE program manager, or representative, provided market penetration point estimates. For example, the Program Manager estimated the market penetration to be $15 \%$ in 2020 for quick-fill walls in new single-story buildings. Given that $41.8 \%{ }^{6}$ of commercial buildings are single story, this resulted in a market penetration rate of $6.3 \%$.

These estimates were then used in the previously developed and documented market penetration model (see Appendix C) to estimate the market penetration by year. Pertinent data for the market penetration estimates are provided in Table D.36.

\section{Baseline Assumptions}

Consistent with the NEMS model, the heating and cooling envelope factors were assumed to be decreasing over time. These changes account for technological improvements over time that would occur without the DOE program. The baseline envelope factors in NEMS are modified annually to account for the technological improvements, and the modifiers are calculated using the following equation with 1995 being the base year:

$$
\text { Baseline Modifier }{ }_{\text {new buildings }}=0.94^{\left(\frac{\text { CurrentYear-1995 }}{25}\right)}
$$

and

$$
\text { Baseline Modifier }{ }_{\text {existing buildings }}=0.96^{\left(\frac{\text { CurrentYear-1995 }}{25}\right)}
$$

\footnotetext{
21995 CBECS, Table 9. Where no data were available, expert judgment was used.

31997 RECS, Table HC1-4b, single-family.

41997 RECS, Table HC1-4b, five or more units.

5 http://www.nmhc.org/research/default.html.

${ }^{6} 1995$ CBECS, Table 9.
} 
The program benefits are in addition to the baseline modifier.

\section{Special Considerations}

For the electrochromic windows program, 30\% of the lighting energy used in commercial buildings is also assumed to be saved. These savings occur at the same rate as the penetration of the electrochromic technology.

\section{Output}

The output for each technology has nine columns of output containing the following information.

- census division

- building type

- year

- total heating envelope factor adjustment for new buildings

- total cooling envelope factor adjustment for new buildings

- total heating envelope factor adjustment for existing buildings

- total cooling envelope factor adjustment for existing buildings

- lighting load adjustment for new buildings

- lighting load adjustment for existing buildings.

\section{References}

Arthur D. Little, Inc (ADL). 1998. "EIA Technology Forecast Updates-Residential and Commercial Building Technologies, Reference Case."

"Commercial Buildings Energy Consumption Survey." 1995. U.S. Department of Energy, Energy Information Administration. eia.doc.gov/emeu/cbecs/contents.html

Decision Analysis Corporation. 1996. Lighting System Technology Characterization for the NEMS Commercial Sector Demand Module. Final Report, Subtask 2-1-b.

Energy Information Administration. 2000. Annual Energy Outlook. U.S. Department of Energy. Energy Information Administration. 2001. Annual Energy Outlook. U.S. Department of Energy. Pacific Northwest National Laboratory. 1998. Facility Energy Decision System User's Guide, Release 4.0. PNNL-10542, Rev 2.

"Residential Energy Consumption Survey." 1997. U.S. Department of Energy, Energy Information Administration. eia.doc.gov/emeu/recs/contents.html

Vineyard, E.A. and J.R. Sand. 1998. "Fridge of the Future: Designing a One Kilowatt-Hour/Day Domestic Refrigerator Freezer." In 1998 ACEEE Summer Study Proceedings.

\section{List of Terms}

ADL Arthur D. Little 


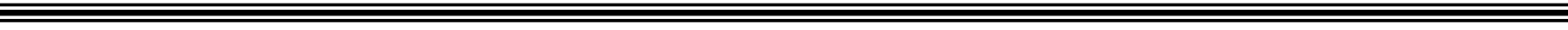

$\begin{array}{ll}\text { AEO } & \text { Annual Energy Outlook (report) } \\ \text { AFUE } & \text { annual fuel utilization efficiency } \\ \text { BTS } & \text { Office of Building Technology, State and Community Programs } \\ \text { CBECS } & \text { Commercial Buildings Energy Consumption Survey } \\ \text { COP } & \text { coefficient of performance } \\ \text { DOE } & \text { U.S. Department of Energy } \\ \text { EER } & \text { energy efficiency ratio } \\ \text { EIA } & \text { Energy Information Administration } \\ \text { FEDS } & \text { Facility Energy Decision System } \\ \text { GPRA } & \text { Government Performance and Results Act of 1993 } \\ \text { LBNL } & \text { Lawrence Berkeley National Laboratory } \\ \text { NEMS } & \text { National Energy Modeling System } \\ \text { ORNL } & \text { Oak Ridge National Laboratory } \\ \text { RECS } & \text { Residential Energy Consumption Survey } \\ \text { SEER } & \text { seasonal energy efficiency ratio }\end{array}$


Table D.1. Size of the Residential Building Market

\begin{tabular}{|l|l|l|c|c|c|c|c|c|}
\hline Building Type & \multirow{2}{*}{ Vintage } & \multirow{2}{*}{ Region } & \multicolumn{6}{|c|}{ Millions of Households } \\
\cline { 4 - 9 } & & & $\mathbf{2 0 0 1}$ & $\mathbf{2 0 0 5}$ & $\mathbf{2 0 1 0}$ & $\mathbf{2 0 1 5}$ & $\mathbf{2 0 2 0}$ & $\mathbf{2 0 3 0}$ \\
\hline Single Family & New & North & .52 & .52 & .53 & .52 & .51 & .50 \\
\hline Single Family & New & South & .52 & .52 & .53 & .52 & .51 & .50 \\
\hline Mobile Homes & New & North & .14 & .14 & .13 & .12 & .1 & .09 \\
\hline Mobile Homes & New & South & .17 & .17 & .16 & .15 & .13 & .11 \\
\hline Multifamily & New & North & .18 & .18 & .22 & .22 & .19 & .18 \\
\hline Multifamily & New & South & .18 & .18 & .22 & .22 & .19 & .18 \\
\hline Residential & New & All & 1.71 & 1.71 & 1.79 & 1.75 & 1.63 & 1.56 \\
\hline Residential & New & Cumulative & 1.71 & 8.56 & 17.52 & 26.28 & 34.49 & 49.99 \\
\hline
\end{tabular}

Table D.2. Potential Penetration Rates for New Construction

\begin{tabular}{|c|c|c|c|c|c|c|c|c|}
\hline \multirow[t]{2}{*}{ Building Type } & \multirow[t]{2}{*}{ Vintage } & \multirow[t]{2}{*}{ Region } & \multicolumn{6}{|c|}{$\%$ of New Construction in Period } \\
\hline & & & 2001 & 2005 & 2010 & 2015 & 2020 & 2030 \\
\hline Single Family & New & North & .3 & 3.1 & 11.6 & 22.1 & 27 & 28.6 \\
\hline Single Family & New & South & .3 & 3.1 & 11.6 & 22.1 & 27 & 28.6 \\
\hline Mobile Homes & New & North & .1 & 1 & 3.8 & 7.3 & 8.9 & 9.4 \\
\hline Mobile Homes & New & South & .1 & 1 & 3.8 & 7.3 & 8.9 & 9.4 \\
\hline Multifamily & New & North & .1 & .7 & 2.6 & 4.9 & 5.9 & 6.3 \\
\hline Multifamily & New & South & .1 & .7 & 2.6 & 4.9 & 5.9 & 6.3 \\
\hline
\end{tabular}

Table D.3. Number of Units Impacted by the BTS

\begin{tabular}{|c|c|c|c|c|c|c|c|c|}
\hline \multirow[t]{2}{*}{ Building Type } & \multirow[t]{2}{*}{ Vintage } & \multirow[t]{2}{*}{ Region } & \multicolumn{6}{|c|}{ Millions of Households } \\
\hline & & & 2001 & 2005 & 2010 & 2015 & 2020 & 2030 \\
\hline Single Family & New & North & .002 & .016 & .061 & .115 & .138 & .143 \\
\hline Single Family & New & South & .002 & .016 & .061 & .115 & .138 & .143 \\
\hline Mobile Homes & New & North & .0001 & .001 & .005 & .009 & .009 & .008 \\
\hline Mobile Homes & New & South & .0002 & .002 & .006 & .01 & .012 & .010 \\
\hline Multifamily & $\mathrm{New}$ & North & .0002 & .001 & .006 & .011 & .011 & .011 \\
\hline Multifamily & New & South & .0002 & .001 & .006 & .011 & .011 & .011 \\
\hline
\end{tabular}

Table D.4. Cumulative Impacted of Household Units

\begin{tabular}{|l|l|l|c|c|c|c|c|c|}
\hline Building Type & \multirow{2}{*}{ Vintage } & \multicolumn{7}{|c|}{ Millions of Households } \\
\cline { 3 - 9 } & & Region & $\mathbf{2 0 0 1}$ & $\mathbf{2 0 0 5}$ & $\mathbf{2 0 1 0}$ & $\mathbf{2 0 1 5}$ & $\mathbf{2 0 2 0}$ & $\mathbf{2 0 3 0}$ \\
\hline Single Family & New & North & .002 & .040 & .245 & .720 & 1.67 & 2.78 \\
\hline Single Family & New & South & .002 & .040 & .245 & .720 & 1.67 & 2.78 \\
\hline Mobile Homes & New & North & .0001 & .004 & .020 & .056 & .100 & .183 \\
\hline Mobile Homes & New & South & .0002 & .004 & .025 & .069 & .122 & .224 \\
\hline Multifamily & New & North & .0002 & .003 & .022 & .066 & .120 & .230 \\
\hline Multifamily & New & South & .0002 & .003 & .022 & .066 & .120 & .230 \\
\hline
\end{tabular}




\section{D.5. End-Use Loads for Heating, Cooling, and Water Heating for New Residential Buildings (MBtu/household/yr)}

\begin{tabular}{|c|c|c|c|c|c|c|c|c|}
\hline Building Type & Vintage & Region & 2001 & 2005 & 2010 & 2015 & 2020 & 2030 \\
\hline \multicolumn{9}{|c|}{ End Use: Space Heating } \\
\hline Single Family & New & North & 42.24 & 42.24 & 42.24 & 42.24 & 42.24 & 42.24 \\
\hline Single Family & New & South & 12.98 & 12.98 & 12.98 & 12.98 & 12.98 & 12.98 \\
\hline Mobile Homes & New & North & 42.24 & 42.24 & 42.24 & 42.24 & 42.24 & 42.24 \\
\hline Mobile Homes & New & South & 12.98 & 12.98 & 12.98 & 12.98 & 12.98 & 12.98 \\
\hline Multifamily & New & North & 42.24 & 42.24 & 42.24 & 42.24 & 42.24 & 42.24 \\
\hline Multifamily & New & South & 12.98 & 12.98 & 12.98 & 12.98 & 12.98 & 12.98 \\
\hline \multicolumn{9}{|c|}{ End Use: Space Cooling } \\
\hline Single Family & New & North & 8.62 & 8.62 & 8.62 & 8.62 & 8.62 & 8.62 \\
\hline Single Family & New & South & 13.58 & 13.58 & 13.58 & 13.58 & 13.58 & 13.58 \\
\hline Mobile Homes & New & North & 8.62 & 8.62 & 8.62 & 8.62 & 8.62 & 8.62 \\
\hline Mobile Homes & New & South & 13.58 & 13.58 & 13.58 & 13.58 & 13.58 & 13.58 \\
\hline Multifamily & New & North & 8.62 & 8.62 & 8.62 & 8.62 & 8.62 & 8.62 \\
\hline Multifamily & New & South & 13.58 & 13.58 & 13.58 & 13.58 & 13.58 & 13.58 \\
\hline \multicolumn{9}{|c|}{ End Use: Water Heating } \\
\hline Single Family & New & North & 12.94 & 12.94 & 12.94 & 12.94 & 12.94 & 12.94 \\
\hline Single Family & New & South & 12.94 & 12.94 & 12.94 & 12.94 & 12.94 & 12.94 \\
\hline Mobile Homes & New & North & 12.94 & 12.94 & 12.94 & 12.94 & 12.94 & 12.94 \\
\hline Mobile Homes & New & South & 12.94 & 12.94 & 12.94 & 12.94 & 12.94 & 12.94 \\
\hline Multifamily & New & North & 12.94 & 12.94 & 12.94 & 12.94 & 12.94 & 12.94 \\
\hline Multifamily & New & South & 12.94 & 12.94 & 12.94 & 12.94 & 12.94 & 12.94 \\
\hline
\end{tabular}

Table D.6. Percent Load Reductions for the Residential Building R\&D Program for Space Heating and Cooling and Water Heating

\begin{tabular}{|c|c|c|c|c|c|c|c|c|}
\hline \multirow[t]{2}{*}{ Building Type } & \multirow[t]{2}{*}{ Vintage } & \multirow[t]{2}{*}{ Region } & \multicolumn{6}{|c|}{$\%$ Load Reduction } \\
\hline & & & 2001 & 2005 & 2010 & 2015 & 2020 & 2030 \\
\hline Single Family & $\mathrm{New}$ & North & 38 & 50 & 50 & 50 & 50 & 50 \\
\hline Single Family & New & South & 38 & 50 & 50 & 50 & 50 & 50 \\
\hline Mobile Homes & New & North & 38 & 50 & 50 & 50 & 50 & 50 \\
\hline Mobile Homes & New & South & 38 & 50 & 50 & 50 & 50 & 50 \\
\hline Multifamily & New & North & 38 & 50 & 50 & 50 & 50 & 50 \\
\hline Multifamily & New & South & 38 & 50 & 50 & 50 & 50 & 50 \\
\hline
\end{tabular}


Table D.7. Building-Level Load Reductions by End Use (MMBtu/household/yr)

\begin{tabular}{|l|l|l|l|l|l|l|l|l|l|}
\hline Building Type & Vintage & Region & $\mathbf{2 0 0 1}$ & $\mathbf{2 0 0 5}$ & $\mathbf{2 0 1 0}$ & $\mathbf{2 0 1 5}$ & $\mathbf{2 0 2 0}$ & $\mathbf{2 0 3 0}$ \\
\hline End Use: Space Heating \\
\hline Single Family & New & North & 16.05 & 21.12 & 21.12 & 21.12 & 21.12 & 21.12 \\
\hline Single Family & New & South & 4.93 & 2.465 & 2.465 & 2.465 & 2.465 & 2.465 \\
\hline Mobile Homes & New & North & 16.05 & 21.12 & 21.12 & 21.12 & 21.12 & 21.12 \\
\hline Mobile Homes & New & South & 4.93 & 2.465 & 2.465 & 2.465 & 2.465 & 2.465 \\
\hline Multifamily & New & North & 16.05 & 21.12 & 21.12 & 21.12 & 21.12 & 21.12 \\
\hline Multifamily & New & South & 4.93 & 2.465 & 2.465 & 2.465 & 2.465 & 2.465 \\
\hline End Use: Space Cooling \\
\hline \multicolumn{7}{|l|l|l|l|}{} \\
\hline Single Family & New & North & 3.27 & 4.31 & 4.31 & 4.31 & 4.31 & 4.31 \\
\hline Single Family & New & South & 5.16 & 6.79 & 6.79 & 6.79 & 6.79 & 6.79 \\
\hline Mobile Homes & New & North & 3.27 & 4.31 & 4.31 & 4.31 & 4.31 & 4.31 \\
\hline Mobile Homes & New & South & 5.16 & 6.79 & 6.79 & 6.79 & 6.79 & 6.79 \\
\hline Multifamily & New & North & 3.27 & 4.31 & 4.31 & 4.31 & 4.31 & 4.31 \\
\hline Multifamily & New & South & 5.16 & 6.79 & 6.79 & 6.79 & 6.79 & 6.79 \\
\hline End Use: Water Heating & \multicolumn{7}{|l|}{} \\
\hline Single Family & New & North & 4.92 & 6.47 & 6.47 & 6.47 & 6.47 & 6.47 \\
\hline Single Family & New & South & 4.92 & 6.47 & 6.47 & 6.47 & 6.47 & 6.47 \\
\hline Mobile Homes & New & North & 4.92 & 6.47 & 6.47 & 6.47 & 6.47 & 6.47 \\
\hline Mobile Homes & New & South & 4.92 & 6.47 & 6.47 & 6.47 & 6.47 & 6.47 \\
\hline Multifamily & New & North & 4.92 & 6.47 & 6.47 & 6.47 & 6.47 & 6.47 \\
\hline Multifamily & New & South & 4.92 & 6.47 & 6.47 & 6.47 & 6.47 & 6.47 \\
\hline
\end{tabular}

Table D.8. Regional Load Reductions by End Use (TBtu/yr)

\begin{tabular}{|l|l|l|c|c|c|c|c|c|c|}
\hline Building Type & Vintage & Region & $\mathbf{2 0 0 1}$ & $\mathbf{2 0 0 5}$ & $\mathbf{2 0 1 0}$ & $\mathbf{2 0 1 5}$ & $\mathbf{2 0 2 0}$ & $\mathbf{2 0 3 0}$ \\
\hline End Use: Space Heating \\
\hline Single Family & New & North & .03 & .84 & 5.17 & 15.21 & 35.27 & 58.71 \\
\hline Single Family & New & South & .01 & .10 & .60 & 1.77 & 4.11 & 6.85 \\
\hline Mobile Homes & New & North & .002 & .08 & .42 & 1.18 & 2.11 & 3.86 \\
\hline Mobile Homes & New & South & .001 & .01 & .06 & .17 & .30 & .55 \\
\hline Multifamily & New & North & .003 & .06 & .46 & 1.39 & 2.53 & 4.86 \\
\hline Multifamily & New & South & .001 & .007 & .05 & .16 & .30 & .57 \\
\hline End Use: Space Cooling & \multicolumn{7}{|l|}{} \\
\hline Single Family & New & North & .01 & .17 & 1.06 & 3.10 & 7.20 & 11.98 \\
\hline Single Family & New & South & .01 & .27 & 1.66 & 4.89 & 11.34 & 18.88 \\
\hline Mobile Homes & New & North & .0003 & .02 & .09 & .24 & .43 & .79 \\
\hline Mobile Homes & New & South & .001 & .03 & .17 & .47 & .83 & 1.52 \\
\hline Multifamily & New & North & .0007 & .01 & .09 & .28 & .52 & .99 \\
\hline Multifamily & New & South & .001 & .02 & .15 & .45 & .81 & 1.56 \\
\hline End Use: Water Heating & \multicolumn{7}{|l|}{} \\
\hline Single Family & New & North & .01 & .26 & 1.59 & 4.66 & 10.8 & 17.99 \\
\hline Single Family & New & South & .01 & .26 & 1.59 & 4.66 & 10.8 & 17.99 \\
\hline Mobile Homes & New & North & .001 & .03 & .13 & .36 & .65 & 1.18 \\
\hline Mobile Homes & New & South & .001 & .03 & .16 & .45 & .79 & 1.15 \\
\hline Multifamily & New & North & .001 & .02 & .14 & .43 & .78 & 1.49 \\
\hline Multifamily & New & South & .001 & .02 & .14 & .43 & .78 & 1.49 \\
\hline
\end{tabular}


Table D.9. Cumulative Regional Load Reductions (TBtu/yr)

\begin{tabular}{|c|c|c|c|c|c|c|c|c|}
\hline $\begin{array}{c}\text { Building } \\
\text { Type }\end{array}$ & Vintage & Region & 2001 & 2005 & 2010 & 2015 & 2020 & 2030 \\
\hline \multicolumn{9}{|c|}{ End Use: Space Heating } \\
\hline Single Family & New & North & 0.025 & 0.798 & 5.127 & 15.154 & 28.822 & 58.601 \\
\hline Single Family & New & South & 0.010 & 0.319 & 2.051 & 6.063 & 11.532 & 23.446 \\
\hline Mobile Homes & New & North & 0.002 & 0.070 & 0.423 & 1.181 & 2.107 & 3.871 \\
\hline Mobile Homes & New & South & 0.001 & 0.034 & 0.207 & 0.577 & 1.030 & 1.893 \\
\hline Multifamily & New & North & 0.003 & 0.066 & 0.459 & 1.393 & 2.540 & 4.839 \\
\hline Multifamily & New & South & 0.001 & 0.026 & 0.184 & 0.557 & 1.016 & 1.936 \\
\hline \multicolumn{9}{|c|}{ End Use: Space Cooling } \\
\hline Single Family & New & North & 0.005 & 0.163 & 1.046 & 3.093 & 5.882 & 11.959 \\
\hline Single Family & New & South & 0.008 & 0.257 & 1.648 & 4.872 & 9.266 & 18.840 \\
\hline Mobile Homes & New & North & 0.000 & 0.014 & 0.086 & 0.241 & 0.430 & 0.790 \\
\hline Mobile Homes & New & South & 0.001 & 0.028 & 0.166 & 0.464 & 0.828 & 1.521 \\
\hline Multifamily & New & North & 0.001 & 0.013 & 0.094 & 0.284 & 0.518 & 0.987 \\
\hline Multifamily & New & South & 0.001 & 0.021 & 0.148 & 0.448 & 0.817 & 1.556 \\
\hline \multicolumn{9}{|c|}{ End Use: Water Heating } \\
\hline Single Family & New & North & 0.008 & 0.245 & 1.571 & 4.642 & 8.830 & 17.952 \\
\hline Single Family & New & South & 0.008 & 0.245 & 1.571 & 4.642 & 8.830 & 17.952 \\
\hline Mobile Homes & New & North & 0.001 & 0.022 & 0.130 & 0.362 & 0.646 & 1.186 \\
\hline Mobile Homes & New & South & 0.001 & 0.026 & 0.158 & 0.442 & 0.789 & 1.449 \\
\hline Multifamily & New & North & 0.001 & 0.020 & 0.141 & 0.427 & 0.778 & 1.482 \\
\hline Multifamily & New & South & 0.001 & 0.020 & 0.141 & 0.427 & 0.778 & 1.482 \\
\hline
\end{tabular}

Table D.10 Existing Equipment Efficiencies

\begin{tabular}{|l|l|l|l|c|c|c|c|c|c|}
\hline End Use & $\begin{array}{l}\text { Fuel } \\
\text { Type }\end{array}$ & \multicolumn{1}{|c|}{$\begin{array}{c}\text { Equipment } \\
\text { Type }\end{array}$} & Units* & $\mathbf{2 0 0 1}$ & $\mathbf{2 0 0 5}$ & $\mathbf{2 0 1 0}$ & $\mathbf{2 0 1 5}$ & $\mathbf{2 0 2 0}$ & $\mathbf{2 0 3 0}$ \\
\hline Space Heat & Elec & Heat Pump & COP & 1.99 & 1.99 & 1.99 & 1.99 & 1.99 & 1.99 \\
\hline Space Heat & Elec & Forced Air & AFUE & 1 & 1 & 1 & 1 & 1 & 1 \\
\hline Space Heat & Gas & Furnace & AFUE & .78 & .78 & .78 & .78 & .78 & .78 \\
\hline Space Heat & Oil & Furnace & AFUE & .78 & .78 & .78 & .78 & .78 & .78 \\
\hline Space Heat & Gas & $\begin{array}{l}\text { High-Efficiency } \\
\text { Furnace }\end{array}$ & AFUE & .92 & .92 & .92 & .92 & .92 & .92 \\
\hline Space Heat & Gas & Heat Pump & COP & 1.4 & 1.4 & 1.4 & 1.4 & 1.4 & 1.4 \\
\hline Space Cool & Elec & Heat Pump & COP & 2.93 & 2.93 & 2.93 & 2.93 & 2.93 & 2.93 \\
\hline Space Cool & Elec & $\begin{array}{l}\text { Central Air } \\
\text { Conditioning }\end{array}$ & COP & 3.02 & 3.02 & 3.02 & 3.02 & 3.02 & 3.02 \\
\hline Space Cool & Elec & Room Air & COP & 2.64 & 2.64 & 2.64 & 2.64 & 2.64 & 2.64 \\
\hline Space Cool & Gas & Heat Pump & COP & .95 & .95 & .95 & .95 & .95 & .95 \\
\hline Water Heat & Elec & Storage & Effic & .89 & .93 & .93 & .93 & .93 & .93 \\
\hline Water Heat & Gas & Storage & Effic & .55 & .62 & .62 & .62 & .62 & .62 \\
\hline Water Heat & Oil & Storage & Effic & .55 & .55 & .56 & .56 & .56 & .56 \\
\hline${ }^{*}$ COP = coefficient of performance; AFUE $=$ annual fuel utilization efficiency; Effic = efficiency. \\
\hline
\end{tabular}


Table D.11. Potential Energy Savings (TBtu/yr)

\begin{tabular}{|c|c|c|c|c|c|c|c|}
\hline Building Type & Region & 2001 & 2005 & 2010 & 2015 & 2020 & 2030 \\
\hline \multicolumn{8}{|c|}{ Space Heating: Electric Heat Pump } \\
\hline Single Family & North & 0.013 & 0.401 & 2.576 & 7.615 & 14.484 & 29.448 \\
\hline Single Family & South & 0.005 & 0.161 & 1.031 & 3.047 & 5.795 & 11.782 \\
\hline Mobile Homes & North & 0.001 & 0.035 & 0.213 & 0.593 & 1.059 & 1.945 \\
\hline Mobile Homes & South & 0.001 & 0.017 & 0.104 & 0.290 & 0.518 & 0.951 \\
\hline Multifamily & North & 0.001 & 0.033 & 0.231 & 0.700 & 1.276 & 2.432 \\
\hline Multifamily & South & 0.001 & 0.013 & 0.092 & 0.280 & 0.511 & 0.973 \\
\hline \multicolumn{8}{|c|}{ Space Heating: Electric Forced Air } \\
\hline Single Family & North & 0.02 & 0.80 & 5.13 & 15.15 & 28.82 & 58.60 \\
\hline Single Family & South & 0.01 & 0.32 & 2.05 & 6.06 & 11.53 & 23.45 \\
\hline Mobile Homes & North & 0.00 & 0.07 & 0.42 & 1.18 & 2.11 & 3.87 \\
\hline Mobile Homes & South & 0.00 & 0.03 & 0.21 & 0.58 & 1.03 & 1.89 \\
\hline Multifamily & North & 0.00 & 0.07 & 0.46 & 1.39 & 2.54 & 4.84 \\
\hline Multifamily & South & 0.00 & 0.03 & 0.18 & 0.56 & 1.02 & 1.94 \\
\hline \multicolumn{8}{|c|}{ Space Heating: Gas Furnace } \\
\hline Single Family & North & 0.03 & 1.02 & 6.57 & 19.43 & 36.95 & 75.13 \\
\hline Single Family & South & 0.01 & 0.41 & 2.63 & 7.77 & 14.78 & 30.06 \\
\hline Mobile Homes & North & 0.00 & 0.09 & 0.54 & 1.51 & 2.70 & 4.96 \\
\hline Mobile Homes & South & 0.00 & 0.04 & 0.27 & 0.74 & 1.32 & 2.43 \\
\hline Multifamily & North & 0.00 & 0.08 & 0.59 & 1.79 & 3.26 & 6.20 \\
\hline Multifamily & South & 0.00 & 0.03 & 0.24 & 0.71 & 1.30 & 2.48 \\
\hline \multicolumn{8}{|c|}{ Space Heating: Oil Furnace } \\
\hline Single Family & North & 0.03 & 1.02 & 6.57 & 19.43 & 36.95 & 75.13 \\
\hline Single Family & South & 0.01 & 0.41 & 2.63 & 7.77 & 14.78 & 30.06 \\
\hline Mobile Homes & North & 0.00 & 0.09 & 0.54 & 1.51 & 2.70 & 4.96 \\
\hline Mobile Homes & South & 0.00 & 0.04 & 0.27 & 0.74 & 1.32 & 2.43 \\
\hline Multifamily & North & 0.00 & 0.08 & 0.59 & 1.79 & 3.26 & 6.20 \\
\hline Multifamily & South & 0.00 & 0.03 & 0.24 & 0.71 & 1.30 & 2.48 \\
\hline \multicolumn{8}{|c|}{ Space Heating: High-Efficiency Gas Furnace } \\
\hline Single Family & North & 0.03 & 0.87 & 5.57 & 16.47 & 31.33 & 63.70 \\
\hline Single Family & South & 0.01 & 0.35 & 2.23 & 6.59 & 12.53 & 25.48 \\
\hline Mobile Homes & North & 0.00 & 0.08 & 0.46 & 1.28 & 2.29 & 4.21 \\
\hline Mobile Homes & South & 0.00 & 0.04 & 0.22 & 0.63 & 1.12 & 2.06 \\
\hline Multifamily & North & 0.00 & 0.07 & 0.50 & 1.51 & 2.76 & 5.26 \\
\hline Multifamily & South & 0.00 & 0.03 & 0.20 & 0.61 & 1.10 & 2.10 \\
\hline \multicolumn{8}{|c|}{ Space Heating: Gas Heat Pump } \\
\hline Single Family & North & 0.02 & 0.57 & 3.66 & 10.82 & 20.59 & 41.86 \\
\hline Single Family & South & 0.01 & 0.23 & 1.47 & 4.33 & 8.24 & 16.75 \\
\hline Mobile Homes & North & 0.00 & 0.05 & 0.30 & 0.84 & 1.51 & 2.77 \\
\hline Mobile Homes & South & 0.00 & 0.02 & 0.15 & 0.41 & 0.74 & 1.35 \\
\hline Multifamily & North & 0.00 & 0.05 & 0.33 & 0.99 & 1.81 & 3.46 \\
\hline Multifamily & South & 0.00 & 0.02 & 0.13 & 0.40 & 0.73 & 1.38 \\
\hline \multicolumn{8}{|c|}{ Space Cooling: Heat Pump } \\
\hline Single Family & North & 0.00 & 0.06 & 0.36 & 1.06 & 2.01 & 4.08 \\
\hline Single Family & South & 0.00 & 0.09 & 0.56 & 1.66 & 3.16 & 6.43 \\
\hline Mobile Homes & North & 0.00 & 0.00 & 0.03 & 0.08 & 0.15 & 0.27 \\
\hline Mobile Homes & South & 0.00 & 0.01 & 0.06 & 0.16 & 0.28 & 0.52 \\
\hline Multifamily & North & 0.00 & 0.00 & 0.03 & 0.10 & 0.18 & 0.34 \\
\hline Multifamily & South & 0.00 & 0.01 & 0.05 & 0.15 & 0.28 & 0.53 \\
\hline \multicolumn{8}{|c|}{ Space Cooling: Central Air Conditioning } \\
\hline Single Family & North & 0.00 & 0.04 & 0.27 & 0.79 & 1.51 & 3.07 \\
\hline Single Family & South & 0.00 & 0.07 & 0.42 & 1.25 & 2.38 & 4.83 \\
\hline
\end{tabular}




\begin{tabular}{|c|c|c|c|c|c|c|c|}
\hline Building Type & Region & 2001 & 2005 & 2010 & 2015 & 2020 & 2030 \\
\hline Mobile Homes & North & 0.00 & 0.00 & 0.02 & 0.06 & 0.11 & 0.20 \\
\hline Mobile Homes & South & 0.00 & 0.01 & 0.04 & 0.12 & 0.21 & 0.39 \\
\hline Multifamily & North & 0.00 & 0.00 & 0.02 & 0.07 & 0.13 & 0.25 \\
\hline Multifamily & South & 0.00 & 0.01 & 0.04 & 0.11 & 0.21 & 0.40 \\
\hline \multicolumn{8}{|c|}{ Space Cooling: Room Air } \\
\hline Single Family & North & 0.00 & 0.06 & 0.40 & 1.17 & 2.23 & 4.53 \\
\hline Single Family & South & 0.00 & 0.10 & 0.62 & 1.85 & 3.51 & 7.14 \\
\hline Mobile Homes & North & 0.00 & 0.01 & 0.03 & 0.09 & 0.16 & 0.30 \\
\hline Mobile Homes & South & 0.00 & 0.01 & 0.06 & 0.18 & 0.31 & 0.58 \\
\hline Multifamily & North & 0.00 & 0.01 & 0.04 & 0.11 & 0.20 & 0.37 \\
\hline Multifamily & South & 0.00 & 0.01 & 0.06 & 0.17 & 0.31 & 0.59 \\
\hline \multicolumn{8}{|c|}{ Space Cooling: Gas Heat Pump } \\
\hline Single Family & North & 0.01 & 0.17 & 1.10 & 3.26 & 6.19 & 12.59 \\
\hline Single Family & South & 0.01 & 0.27 & 1.73 & 5.13 & 9.75 & 19.83 \\
\hline Mobile Homes & North & 0.00 & 0.02 & 0.09 & 0.25 & 0.45 & 0.83 \\
\hline Mobile Homes & South & 0.00 & 0.03 & 0.18 & 0.49 & 0.87 & 1.60 \\
\hline Multifamily & North & 0.00 & 0.01 & 0.10 & 0.30 & 0.55 & 1.04 \\
\hline Multifamily & South & 0.00 & 0.02 & 0.16 & 0.47 & 0.86 & 1.64 \\
\hline \multicolumn{8}{|c|}{ Water Heat: Electric } \\
\hline Single Family & North & 0.01 & 0.26 & 1.69 & 4.99 & 9.49 & 19.30 \\
\hline Single Family & South & 0.01 & 0.26 & 1.69 & 4.99 & 9.49 & 19.30 \\
\hline Mobile Homes & North & 0.00 & 0.02 & 0.14 & 0.39 & 0.69 & 1.28 \\
\hline Mobile Homes & South & 0.00 & 0.03 & 0.17 & 0.48 & 0.85 & 1.56 \\
\hline Multifamily & North & 0.00 & 0.02 & 0.15 & 0.46 & 0.84 & 1.59 \\
\hline Multifamily & South & 0.00 & 0.02 & 0.15 & 0.46 & 0.84 & 1.59 \\
\hline \multicolumn{8}{|c|}{ Water Heat: Gas } \\
\hline Single Family & North & 0.01 & 0.39 & 2.53 & 7.49 & 14.24 & 28.96 \\
\hline Single Family & South & 0.01 & 0.39 & 2.53 & 7.49 & 14.24 & 28.96 \\
\hline Mobile Homes & North & 0.00 & 0.03 & 0.21 & 0.58 & 1.04 & 1.91 \\
\hline Mobile Homes & South & 0.00 & 0.04 & 0.26 & 0.71 & 1.27 & 2.34 \\
\hline Multifamily & North & 0.00 & 0.03 & 0.23 & 0.69 & 1.26 & 2.39 \\
\hline Multifamily & South & 0.00 & 0.03 & 0.23 & 0.69 & 1.26 & 2.39 \\
\hline \multicolumn{8}{|c|}{ Water Heat: Oil } \\
\hline Single Family & North & 0.01 & 0.44 & 2.80 & 8.29 & 15.77 & 32.06 \\
\hline Single Family & South & 0.01 & 0.44 & 2.80 & 8.29 & 15.77 & 32.06 \\
\hline Mobile Homes & North & 0.00 & 0.04 & 0.23 & 0.65 & 1.15 & 2.12 \\
\hline Mobile Homes & South & 0.00 & 0.05 & 0.28 & 0.79 & 1.41 & 2.59 \\
\hline Multifamily & North & 0.00 & 0.04 & 0.25 & 0.76 & 1.39 & 2.65 \\
\hline Multifamily & South & 0.00 & 0.04 & 0.25 & 0.76 & 1.39 & 2.65 \\
\hline
\end{tabular}


Table D.12. Equipment Market Shares (\%)

\begin{tabular}{|c|c|c|c|c|c|c|c|}
\hline Building Type & Region & 2001 & 2005 & 2010 & 2015 & 2020 & 2030 \\
\hline \multicolumn{8}{|c|}{ Space Heating: Electric Heat Pump } \\
\hline Single Family & North & 13 & 14 & 15 & 16 & 17 & 17 \\
\hline Single Family & South & 27 & 28 & 29 & 30 & 32 & 32 \\
\hline Mobile Homes & North & 13 & 14 & 15 & 16 & 17 & 17 \\
\hline Mobile Homes & South & 27 & 28 & 29 & 30 & 32 & 32 \\
\hline Multifamily & North & 13 & 14 & 15 & 16 & 17 & 17 \\
\hline Multifamily & South & 27 & 28 & 29 & 30 & 32 & 32 \\
\hline \multicolumn{8}{|c|}{ Space Heating: Electric Forced Air } \\
\hline Single Family & North & 7 & 8 & 9 & 10 & 11 & 11 \\
\hline Single Family & South & 12 & 13 & 15 & 17 & 19 & 19 \\
\hline Mobile Homes & North & 7 & 8 & 9 & 10 & 11 & 11 \\
\hline Mobile Homes & South & 12 & 13 & 15 & 17 & 19 & 19 \\
\hline Multifamily & North & 7 & 8 & 9 & 10 & 11 & 11 \\
\hline Multifamily & South & 12 & 13 & 15 & 17 & 19 & 19 \\
\hline \multicolumn{8}{|c|}{ Space Heating: Gas Furnace } \\
\hline Single Family & North & 56 & 52 & 47 & 41 & 36 & 36 \\
\hline Single Family & South & 47 & 43 & 38 & 32 & 27 & 27 \\
\hline Mobile Homes & North & 56 & 52 & 47 & 41 & 36 & 36 \\
\hline Mobile Homes & South & 47 & 43 & 38 & 32 & 27 & 27 \\
\hline Multifamily & North & 56 & 52 & 47 & 41 & 36 & 36 \\
\hline Multifamily & South & 47 & 43 & 38 & 32 & 27 & 27 \\
\hline \multicolumn{8}{|c|}{ Space Heating: Oil Furnace } \\
\hline Single Family & North & 3 & 3 & 3 & 3 & 3 & 3 \\
\hline Single Family & South & 1 & 1 & 1 & 1 & 1 & 1 \\
\hline Mobile Homes & North & 3 & 3 & 3 & 3 & 3 & 3 \\
\hline Mobile Homes & South & 1 & 1 & 1 & 1 & 1 & 1 \\
\hline Multifamily & North & 3 & 3 & 3 & 3 & 3 & 3 \\
\hline Multifamily & South & 1 & 1 & 1 & 1 & 1 & 1 \\
\hline \multicolumn{8}{|c|}{ Space Heating: High-Efficiency Gas Furnace } \\
\hline Single Family & North & 15 & 17 & 20 & 23 & 26 & 26 \\
\hline Single Family & South & 12 & 14 & 16 & 18 & 20 & 20 \\
\hline Mobile Homes & North & 15 & 17 & 20 & 23 & 26 & 26 \\
\hline Mobile Homes & South & 12 & 14 & 16 & 18 & 20 & 20 \\
\hline Multifamily & North & 15 & 17 & 20 & 23 & 26 & 26 \\
\hline Multifamily & South & 12 & 14 & 16 & 18 & 20 & 20 \\
\hline \multicolumn{8}{|c|}{ Space Heating: Gas Heat Pump } \\
\hline Single Family & North & 2 & 2 & 2 & 2 & 2 & 2 \\
\hline Single Family & South & 1 & 0 & 0 & 0 & 0 & 0 \\
\hline Mobile Homes & North & 2 & 2 & 2 & 2 & 2 & 2 \\
\hline Mobile Homes & South & 1 & 0 & 0 & 0 & 0 & 0 \\
\hline Multifamily & North & 2 & 2 & 2 & 2 & 2 & 2 \\
\hline Multifamily & South & 1 & 0 & 0 & 0 & 0 & 0 \\
\hline \multicolumn{8}{|c|}{ Space Cooling: Heat Pump } \\
\hline Single Family & North & 13 & 14 & 15 & 16 & 17 & 17 \\
\hline Single Family & South & 27 & 28 & 29 & 30 & 32 & 32 \\
\hline Mobile Homes & North & 13 & 14 & 15 & 16 & 17 & 17 \\
\hline Mobile Homes & South & 27 & 28 & 29 & 30 & 32 & 32 \\
\hline Multifamily & North & 13 & 14 & 15 & 16 & 17 & 17 \\
\hline Multifamily & South & 27 & 28 & 29 & 30 & 32 & 32 \\
\hline \multicolumn{8}{|c|}{ Space Cooling: Central Air Conditioning } \\
\hline Single Family & North & 56 & 55 & 54 & 53 & 52 & 52 \\
\hline Single Family & South & 58 & 57 & 55 & 54 & 53 & 53 \\
\hline
\end{tabular}




\begin{tabular}{|c|c|c|c|c|c|c|c|}
\hline Building Type & Region & 2001 & 2005 & 2010 & 2015 & 2020 & 2030 \\
\hline Mobile Homes & North & 56 & 55 & 54 & 53 & 52 & 52 \\
\hline Mobile Homes & South & 58 & 57 & 55 & 54 & 53 & 53 \\
\hline Multifamily & North & 56 & 55 & 54 & 53 & 52 & 52 \\
\hline Multifamily & South & 58 & 57 & 55 & 54 & 53 & 53 \\
\hline \multicolumn{8}{|c|}{ Space Cooling: Room Air } \\
\hline Single Family & North & 3 & 3 & 2 & 2 & 2 & 2 \\
\hline Single Family & South & 1 & 1 & 1 & 1 & 1 & 1 \\
\hline Mobile Homes & North & 3 & 3 & 2 & 2 & 2 & 2 \\
\hline Mobile Homes & South & 1 & 1 & 1 & 1 & 1 & 1 \\
\hline Multifamily & North & 3 & 3 & 2 & 2 & 2 & 2 \\
\hline Multifamily & South & 1 & 1 & 1 & 1 & 1 & 1 \\
\hline \multicolumn{8}{|c|}{ Space Cooling: Gas Heat Pump } \\
\hline Single Family & North & 2 & 2 & 2 & 2 & 2 & 2 \\
\hline Single Family & South & 1 & 0 & 0 & 0 & 0 & 0 \\
\hline Mobile Homes & North & 2 & 2 & 2 & 2 & 2 & 2 \\
\hline Mobile Homes & South & 1 & 0 & 0 & 0 & 0 & 0 \\
\hline Multifamily & North & 2 & 2 & 2 & 2 & 2 & 2 \\
\hline Multifamily & South & 1 & 0 & 0 & 0 & 0 & 0 \\
\hline \multicolumn{8}{|c|}{ Water Heat: Electric } \\
\hline Single Family & North & 23 & 23 & 22 & 22 & 22 & 22 \\
\hline Single Family & South & 23 & 23 & 22 & 22 & 22 & 22 \\
\hline Mobile Homes & North & 23 & 23 & 22 & 22 & 22 & 22 \\
\hline Mobile Homes & South & 23 & 23 & 22 & 22 & 22 & 22 \\
\hline Multifamily & North & 23 & 23 & 22 & 22 & 22 & 22 \\
\hline Multifamily & South & 23 & 23 & 22 & 22 & 22 & 22 \\
\hline \multicolumn{8}{|c|}{ Water Heat: Gas } \\
\hline Single Family & North & 73 & 73 & 73 & 74 & 74 & 74 \\
\hline Single Family & South & 73 & 73 & 73 & 74 & 74 & 74 \\
\hline Mobile Homes & North & 73 & 73 & 73 & 74 & 74 & 74 \\
\hline Mobile Homes & South & 73 & 73 & 73 & 74 & 74 & 74 \\
\hline Multifamily & North & 73 & 73 & 73 & 74 & 74 & 74 \\
\hline Multifamily & South & 73 & 73 & 73 & 74 & 74 & 74 \\
\hline \multicolumn{8}{|c|}{ Water Heat: Oil } \\
\hline Single Family & North & 5 & 5 & 4 & 4 & 4 & 4 \\
\hline Single Family & South & 5 & 5 & 4 & 4 & 4 & 4 \\
\hline Mobile Homes & North & 5 & 5 & 4 & 4 & 4 & 4 \\
\hline Mobile Homes & South & 5 & 5 & 4 & 4 & 4 & 4 \\
\hline Multifamily & North & 5 & 5 & 4 & 4 & 4 & 4 \\
\hline Multifamily & South & 5 & 5 & 4 & 4 & 4 & 4 \\
\hline
\end{tabular}


Table D.13. Actual Energy Savings (TBtu/yr)

\begin{tabular}{|c|c|c|c|c|c|c|c|}
\hline Building Type & Region & 2001 & 2005 & 2010 & 2015 & 2020 & 2030 \\
\hline \multicolumn{8}{|c|}{ Space Heating: Electric Heat Pump } \\
\hline Single Family & North & 0.00 & 0.06 & 0.39 & 1.22 & 2.46 & 5.01 \\
\hline Single Family & South & 0.00 & 0.04 & 0.30 & 0.91 & 1.85 & 3.77 \\
\hline Mobile Homes & North & 0.00 & 0.00 & 0.03 & 0.09 & 0.18 & 0.33 \\
\hline Mobile Homes & South & 0.00 & 0.00 & 0.03 & 0.09 & 0.17 & 0.30 \\
\hline Multifamily & North & 0.00 & 0.00 & 0.03 & 0.11 & 0.22 & 0.41 \\
\hline Multifamily & South & 0.00 & 0.00 & 0.03 & 0.08 & 0.16 & 0.31 \\
\hline \multicolumn{8}{|c|}{ Space Heating: Electric Forced Air } \\
\hline Single Family & North & 0.00 & 0.06 & 0.46 & 1.52 & 3.17 & 6.45 \\
\hline Single Family & South & 0.00 & 0.04 & 0.31 & 1.03 & 2.19 & 4.45 \\
\hline Mobile Homes & North & 0.00 & 0.01 & 0.04 & 0.12 & 0.23 & 0.43 \\
\hline Mobile Homes & South & 0.00 & 0.00 & 0.03 & 0.10 & 0.20 & 0.36 \\
\hline Multifamily & North & 0.00 & 0.01 & 0.04 & 0.14 & 0.28 & 0.53 \\
\hline Multifamily & South & 0.00 & 0.00 & 0.03 & 0.09 & 0.19 & 0.37 \\
\hline \multicolumn{8}{|c|}{ Space Heating: Gas Furnace } \\
\hline Single Family & North & 0.02 & 0.53 & 3.09 & 7.97 & 13.30 & 27.05 \\
\hline Single Family & South & 0.01 & 0.18 & 1.00 & 2.49 & 3.99 & 8.12 \\
\hline Mobile Homes & North & 0.00 & 0.05 & 0.25 & 0.62 & 0.97 & 1.79 \\
\hline Mobile Homes & South & 0.00 & 0.02 & 0.10 & 0.24 & 0.36 & 0.66 \\
\hline Multifamily & North & 0.00 & 0.04 & 0.28 & 0.73 & 1.17 & 2.23 \\
\hline Multifamily & South & 0.00 & 0.01 & 0.09 & 0.23 & 0.35 & 0.67 \\
\hline \multicolumn{8}{|c|}{ Space Heating: Oil Furnace } \\
\hline Single Family & North & 0.00 & 0.03 & 0.20 & 0.58 & 1.11 & 2.25 \\
\hline Single Family & South & 0.00 & 0.00 & 0.03 & 0.08 & 0.15 & 0.30 \\
\hline Mobile Homes & North & 0.00 & 0.00 & 0.02 & 0.05 & 0.08 & 0.15 \\
\hline Mobile Homes & South & 0.00 & 0.00 & 0.00 & 0.01 & 0.01 & 0.02 \\
\hline Multifamily & North & 0.00 & 0.00 & 0.02 & 0.05 & 0.10 & 0.19 \\
\hline Multifamily & South & 0.00 & 0.00 & 0.00 & 0.01 & 0.01 & 0.02 \\
\hline \multicolumn{8}{|c|}{ Space Heating: High-Efficiency Gas Furnace } \\
\hline Single Family & North & 0.00 & 0.15 & 1.11 & 3.79 & 8.15 & 16.56 \\
\hline Single Family & South & 0.00 & 0.05 & 0.36 & 1.19 & 2.51 & 5.10 \\
\hline Mobile Homes & North & 0.00 & 0.01 & 0.09 & 0.30 & 0.60 & 1.09 \\
\hline Mobile Homes & South & 0.00 & 0.01 & 0.04 & 0.11 & 0.22 & 0.41 \\
\hline Multifamily & North & 0.00 & 0.01 & 0.10 & 0.35 & 0.72 & 1.37 \\
\hline Multifamily & South & 0.00 & 0.00 & 0.03 & 0.11 & 0.22 & 0.42 \\
\hline \multicolumn{8}{|c|}{ Space Heating: Gas Heat Pump } \\
\hline Single Family & North & 0.00 & 0.01 & 0.07 & 0.22 & 0.41 & 0.84 \\
\hline Single Family & South & 0.00 & 0.00 & 0.00 & 0.00 & 0.00 & 0.00 \\
\hline Mobile Homes & North & 0.00 & 0.00 & 0.01 & 0.02 & 0.03 & 0.06 \\
\hline Mobile Homes & South & 0.00 & 0.00 & 0.00 & 0.00 & 0.00 & 0.00 \\
\hline Multifamily & North & 0.00 & 0.00 & 0.01 & 0.02 & 0.04 & 0.07 \\
\hline Multifamily & South & 0.00 & 0.00 & 0.00 & 0.00 & 0.00 & 0.00 \\
\hline \multicolumn{8}{|c|}{ Space Cooling: Heat Pump } \\
\hline Single Family & North & 0.00 & 0.01 & 0.05 & 0.17 & 0.34 & 0.69 \\
\hline Single Family & South & 0.00 & 0.02 & 0.16 & 0.50 & 1.01 & 2.06 \\
\hline Mobile Homes & North & 0.00 & 0.00 & 0.00 & 0.01 & 0.02 & 0.05 \\
\hline Mobile Homes & South & 0.00 & 0.00 & 0.02 & 0.05 & 0.09 & 0.17 \\
\hline Multifamily & North & 0.00 & 0.00 & 0.00 & 0.02 & 0.03 & 0.06 \\
\hline Multifamily & South & 0.00 & 0.00 & 0.01 & 0.05 & 0.09 & 0.17 \\
\hline \multicolumn{8}{|c|}{ Space Cooling: Central Air Conditioning } \\
\hline Single Family & North & 0.00 & 0.02 & 0.14 & 0.42 & 0.78 & 1.59 \\
\hline Single Family & South & 0.00 & 0.04 & 0.23 & 0.67 & 1.26 & 2.56 \\
\hline
\end{tabular}

Appendix D - 24 


\begin{tabular}{|c|c|c|c|c|c|c|c|}
\hline Building Type & Region & 2001 & 2005 & 2010 & 2015 & 2020 & 2030 \\
\hline Mobile Homes & North & 0.00 & 0.00 & 0.01 & 0.03 & 0.06 & 0.11 \\
\hline Mobile Homes & South & 0.00 & 0.00 & 0.02 & 0.06 & 0.11 & 0.21 \\
\hline Multifamily & North & 0.00 & 0.00 & 0.01 & 0.04 & 0.07 & 0.13 \\
\hline Multifamily & South & 0.00 & 0.00 & 0.02 & 0.06 & 0.11 & 0.21 \\
\hline \multicolumn{8}{|c|}{ Space Cooling: Room Air } \\
\hline Single Family & North & 0.00 & 0.00 & 0.01 & 0.02 & 0.04 & 0.09 \\
\hline Single Family & South & 0.00 & 0.00 & 0.01 & 0.02 & 0.04 & 0.07 \\
\hline Mobile Homes & North & 0.00 & 0.00 & 0.00 & 0.00 & 0.00 & 0.01 \\
\hline Mobile Homes & South & 0.00 & 0.00 & 0.00 & 0.00 & 0.00 & 0.01 \\
\hline Multifamily & North & 0.00 & 0.00 & 0.00 & 0.00 & 0.00 & 0.01 \\
\hline Multifamily & South & 0.00 & 0.00 & 0.00 & 0.00 & 0.00 & 0.01 \\
\hline \multicolumn{8}{|c|}{ Space Cooling: Gas Heat Pump } \\
\hline Single Family & North & 0.00 & 0.00 & 0.02 & 0.07 & 0.12 & 0.25 \\
\hline Single Family & South & 0.00 & 0.00 & 0.00 & 0.00 & 0.00 & 0.00 \\
\hline Mobile Homes & North & 0.00 & 0.00 & 0.00 & 0.01 & 0.01 & 0.02 \\
\hline Mobile Homes & South & 0.00 & 0.00 & 0.00 & 0.00 & 0.00 & 0.00 \\
\hline Multifamily & North & 0.00 & 0.00 & 0.00 & 0.01 & 0.01 & 0.02 \\
\hline Multifamily & South & 0.00 & 0.00 & 0.00 & 0.00 & 0.00 & 0.00 \\
\hline \multicolumn{8}{|c|}{ Water Heat: Electric } \\
\hline Single Family & North & 0.00 & 0.06 & 0.37 & 1.10 & 2.09 & 4.25 \\
\hline Single Family & South & 0.00 & 0.06 & 0.37 & 1.10 & 2.09 & 4.25 \\
\hline Mobile Homes & North & 0.00 & 0.01 & 0.03 & 0.09 & 0.15 & 0.28 \\
\hline Mobile Homes & South & 0.00 & 0.01 & 0.04 & 0.10 & 0.19 & 0.34 \\
\hline Multifamily & North & 0.00 & 0.00 & 0.03 & 0.10 & 0.18 & 0.35 \\
\hline Multifamily & South & 0.00 & 0.00 & 0.03 & 0.10 & 0.18 & 0.35 \\
\hline \multicolumn{8}{|c|}{ Water Heat: Gas } \\
\hline Single Family & North & 0.01 & 0.29 & 1.85 & 5.54 & 10.54 & 21.43 \\
\hline Single Family & South & 0.01 & 0.29 & 1.85 & 5.54 & 10.54 & 21.43 \\
\hline Mobile Homes & North & 0.00 & 0.03 & 0.15 & 0.43 & 0.77 & 1.42 \\
\hline Mobile Homes & South & 0.00 & 0.03 & 0.19 & 0.53 & 0.94 & 1.73 \\
\hline Multifamily & North & 0.00 & 0.02 & 0.17 & 0.51 & 0.93 & 1.77 \\
\hline Multifamily & South & 0.00 & 0.02 & 0.17 & 0.51 & 0.93 & 1.77 \\
\hline \multicolumn{8}{|c|}{ Water Heat: Oil } \\
\hline Single Family & North & 0.00 & 0.02 & 0.11 & 0.33 & 0.63 & 1.28 \\
\hline Single Family & South & 0.00 & 0.02 & 0.11 & 0.33 & 0.63 & 1.28 \\
\hline Mobile Homes & North & 0.00 & 0.00 & 0.01 & 0.03 & 0.05 & 0.08 \\
\hline Mobile Homes & South & 0.00 & 0.00 & 0.01 & 0.03 & 0.06 & 0.10 \\
\hline Multifamily & North & 0.00 & 0.00 & 0.01 & 0.03 & 0.06 & 0.11 \\
\hline Multifamily & South & 0.00 & 0.00 & 0.01 & 0.03 & 0.06 & 0.11 \\
\hline
\end{tabular}

Table D.14. Energy Savings by Fuel Type (TBtu/yr)

\begin{tabular}{|l|c|c|c|c|c|c|}
\hline \multicolumn{1}{|c|}{ Fuel Type } & $\mathbf{2 0 0 1}$ & $\mathbf{2 0 0 5}$ & $\mathbf{2 0 1 0}$ & $\mathbf{2 0 1 5}$ & $\mathbf{2 0 2 0}$ & $\mathbf{2 0 3 0}$ \\
\hline Delivered Electricity & 0.0 & 0.5 & 3.3 & 10.2 & 20.3 & 40.7 \\
\hline Primary Electricity & 0.0 & 1.3 & 8.2 & 24.4 & 45.2 & 90.8 \\
\hline Natural Gas & 0.1 & 1.8 & 11.0 & 31.5 & 57.8 & 116.2 \\
\hline Oil & 0.0 & 0.1 & 0.5 & 1.6 & 2.9 & 5.9 \\
\hline Total Primary & 0.1 & 3.2 & 19.7 & 57.5 & 106.0 & 213.0 \\
\hline
\end{tabular}


Table D.15. Refrigerator Efficiency and Costs: AEO 2000

\begin{tabular}{|c|c|c|c|c|c|}
\hline Model & $\begin{array}{c}\text { Initial } \\
\text { Year }\end{array}$ & $\begin{array}{c}\text { Ending } \\
\text { Year }\end{array}$ & $\begin{array}{c}\text { Annual } \\
\text { Consumption } \\
(\mathbf{k W h})\end{array}$ & $\begin{array}{c}\text { Installed } \\
\text { Cost } \\
(\mathbf{1 9 9 8} \mathbf{\$})\end{array}$ & $\begin{array}{c}\text { Retail } \\
\text { Cost } \\
(\mathbf{1 9 9 8} \mathbf{)})\end{array}$ \\
\hline 1 & 1997 & 2001 & 690 & 530 & 480 \\
\hline 1 & 2002 & 2020 & 478 & 530 & 480 \\
\hline 2 & 1997 & 2001 & 660 & 550 & 500 \\
\hline 2 & 2002 & 2020 & 460 & 550 & 500 \\
\hline 3 & 1993 & 2001 & 518 & 850 & 800 \\
\hline 3 & 2002 & 2020 & 460 & 550 & 500 \\
\hline 3 & 2005 & 2020 & 400 & 700 & 650 \\
\hline 4 & 1993 & 2001 & 843 & 1313.8 & 1313.8 \\
\hline 4 & 2002 & 2020 & 577 & 1313.8 & 1313.8 \\
\hline
\end{tabular}

Table D.16. Refrigerator Efficiency and Costs: GPRA Baseline

\begin{tabular}{|l|c|c|c|c|c|}
\hline Model & $\begin{array}{c}\text { Initial } \\
\text { Year }\end{array}$ & $\begin{array}{c}\text { Ending } \\
\text { Year }\end{array}$ & $\begin{array}{c}\text { Annual } \\
\text { Consumption } \\
(\mathbf{k W h})\end{array}$ & $\begin{array}{c}\text { Installed } \\
\text { Cost } \\
(\mathbf{1 9 9 8} \$ \mathbf{)}\end{array}$ & $\begin{array}{c}\text { Retail } \\
\text { Cost } \\
(\mathbf{1 9 9 8} \mathbf{\text { \$) }}\end{array}$ \\
\hline 1 & 1997 & 2001 & 690 & 530 & 480 \\
\hline 1 & 2002 & 2020 & 478 & 580 & 480 \\
\hline 2 & 1997 & 2001 & 660 & 550 & 500 \\
\hline 2 & 2002 & 2020 & 460 & 600 & 550 \\
\hline 3 & 1997 & 2001 & 518 & 850 & 800 \\
\hline 3 & 2002 & 2020 & 460 & 600 & 550 \\
\hline 3 & 2005 & 2020 & 400 & 700 & 650 \\
\hline 4 & 1997 & 2001 & 843 & 1313.8 & 1313.8 \\
\hline 4 & 2002 & 2020 & 577 & 1313.8 & 1313.8 \\
\hline
\end{tabular}

Table D.17. Energy Star Program - Refrigerators (market share of 400-kWh/yr units)

\begin{tabular}{|c|c|c|c|c|}
\hline \multirow{2}{*}{$\begin{array}{c}\text { Census } \\
\text { Division }\end{array}$} & \multicolumn{2}{|c|}{ 2005 } & \multicolumn{2}{c|}{ 2010 } \\
\cline { 2 - 5 } & Baseline & $\begin{array}{c}\text { Energy } \\
\text { Star }\end{array}$ & Baseline & $\begin{array}{c}\text { Energy } \\
\text { Star }\end{array}$ \\
\hline 1 & .0427 & .2068 & .0426 & .2064 \\
\hline 2 & .0409 & .2003 & .0400 & .1971 \\
\hline 3 & .0337 & .1727 & .0329 & .1698 \\
\hline 4 & .0326 & .1687 & .0327 & .1689 \\
\hline 5 & .0342 & .1748 & .0341 & .1744 \\
\hline 6 & .0330 & .1702 & .0329 & .1696 \\
\hline 7 & .0329 & .1698 & .0322 & .1668 \\
\hline 8 & .0355 & .1801 & .0356 & .1805 \\
\hline 9 & .0354 & .1793 & .0357 & .1807 \\
\hline
\end{tabular}


Table D.18. Original and Revised NEMS Inputs for Clothes Washers

\begin{tabular}{|c|c|c|c|c|c|c|}
\hline Original NEMS Inputs \\
\hline Technology & $\begin{array}{c}\text { Start } \\
\text { Yr }\end{array}$ & $\begin{array}{c}\text { End } \\
\text { Yr }\end{array}$ & $\begin{array}{c}\text { Water } \\
\text { Coeff. }\end{array}$ & $\begin{array}{c}\text { Energy } \\
\text { Factor }\end{array}$ & $\begin{array}{c}\text { Installed } \\
\text { Cost }\end{array}$ & Type \\
\hline 1 & 1997 & 2020 & .67 & 2.71 & $\$ 490$ & V-Axis \\
\hline 2 & 1997 & 2004 & .67 & 3.88 & $\$ 645$ & V-Axis \\
\hline 3 & 2005 & 2020 & .67 & 3.88 & $\$ 590$ & V-Axis \\
\hline 4 & 1997 & 2020 & .24 & 4.45 & $\$ 800$ & H-Axis \\
\hline 5 & 2005 & 2020 & .24 & 5.27 & $\$ 800$ & H-Axis \\
\hline 6 & 2015 & 2020 & .24 & 5.44 & $\$ 800$ & H-Axis \\
\hline \multicolumn{7}{|l|}{} \\
\hline Revised NEMS Inputs \\
\hline 1 & 1997 & 2020 & 2 & 2.71 & $\$ 490$ & V-Axis \\
\hline 2 & 1997 & 2004 & 2 & 3.88 & $\$ 645$ & V-Axis \\
\hline 3 & 2005 & 2020 & 2 & 3.88 & $\$ 590$ & V-Axis \\
\hline 4 & 1997 & 2020 & .4 & 4.45 & $\$ 800$ & H-Axis \\
\hline 5 & 2005 & 2020 & .4 & 5.27 & $\$ 800$ & H-Axis \\
\hline 6 & 2015 & 2020 & .4 & 5.44 & $\$ 800$ & H-Axis \\
\hline
\end{tabular}

Table D.19. NEMS-Generated Shares of Clothes Washers by Technology

\begin{tabular}{|c|c|c|c|c|}
\hline \multirow{2}{*}{$\begin{array}{c}\text { Census } \\
\text { Division }\end{array}$} & \multicolumn{2}{|c|}{2005} & \multicolumn{2}{c|}{ 2010 } \\
\cline { 2 - 5 } & Baseline & $\begin{array}{c}\text { Energy } \\
\text { Star }\end{array}$ & Baseline & $\begin{array}{c}\text { Energy } \\
\text { Star }\end{array}$ \\
\hline 1 & .0000 & .0927 & .0000 & .0923 \\
\hline 2 & .0000 & .0904 & .0000 & .0900 \\
\hline 3 & .0000 & .0814 & .0000 & .0804 \\
\hline 4 & .0000 & .0794 & .0000 & .0794 \\
\hline 5 & .0000 & .0813 & .0000 & .0812 \\
\hline 6 & .0000 & .0799 & .0000 & .0797 \\
\hline 7 & .0000 & .0801 & .0000 & .0791 \\
\hline 8 & .0000 & .0831 & .0000 & .0833 \\
\hline 9 & .0000 & .0826 & .0000 & .0830 \\
\hline $\begin{array}{l}\text { Notes: Results shown are for new housing units; replace- } \\
\text { ment shares are generally within 0.5\% of values shown here. }\end{array}$ \\
\hline
\end{tabular}

Table D.20. Key NEMS Inputs for Electric Water Heaters (AEO 2000)

\begin{tabular}{|c|c|c|c|c|l|}
\hline Technology & $\begin{array}{c}\text { Start } \\
\text { Yr }\end{array}$ & $\begin{array}{c}\text { End } \\
\text { Yr }\end{array}$ & $\begin{array}{c}\text { Energy } \\
\text { Factor }\end{array}$ & $\begin{array}{c}\text { Installed } \\
\text { Cost }\end{array}$ & Type \\
\hline 1 & 1997 & 2020 & .86 & $\$ 350$ & Resistance \\
\hline 2 & 1997 & 2020 & .88 & $\$ 350$ & Resistance \\
\hline 3 & 1997 & 2020 & .95 & $\$ 575$ & Resistance \\
\hline 4 & 1997 & 2020 & 2.60 & $\$ 1,025$ & Heat Pump \\
\hline 5 & 1997 & 2020 & 2.00 & $\$ 2,600$ & Heat Pump \\
\hline 6 & 2005 & 2020 & .89 & $\$ 350$ & Resistance \\
\hline 7 & 2005 & 2020 & .96 & $\$ 475$ & Resistance \\
\hline 8 & 2005 & 2020 & 2.00 & $\$ 900$ & Heat Pump \\
\hline 9 & 2015 & 2020 & .90 & $\$ 400$ & Resistance \\
\hline 10 & 2015 & 2020 & .96 & $\$ 425$ & Resistance \\
\hline 11 & 2015 & 2020 & 2.20 & $\$ 800$ & Heat Pump \\
\hline
\end{tabular}


Table D.21. NEMS Results for Energy Star Electric Water Heaters (national market shares for new single-family homes)

\begin{tabular}{|c|c|c|c|c|}
\hline & \multicolumn{2}{|c|}{$\mathbf{2 0 0 5}$} & \multicolumn{2}{c|}{$\mathbf{2 0 1 0}$} \\
\hline $\begin{array}{c}\text { Efficiency } \\
\text { Level }\end{array}$ & Baseline & $\begin{array}{c}\text { Energy } \\
\text { Star }\end{array}$ & Baseline & $\begin{array}{c}\text { Energy } \\
\text { Star }\end{array}$ \\
\hline 0.95 & 0.011 & 0.054 & 0.011 & 0.054 \\
\hline 0.96 & 0.056 & 0.128 & 0.056 & 0.127 \\
\hline Total & 0.0670 & 0.1820 & 0.0670 & 0.1810 \\
\hline
\end{tabular}

Note: Results shown are for new, single-family housing units; replacement shares are generally within $2 \%$ of the values shown here.

Table D.22. Key NEMS Inputs for Gas Water Heaters

\begin{tabular}{|c|l|l|c|c|l|}
\hline Technology & Start Yr & End Yr & $\begin{array}{c}\text { Energy } \\
\text { Factor }\end{array}$ & $\begin{array}{c}\text { Installed } \\
\text { Cost }\end{array}$ & Type \\
\hline 1 & 1997 & 2020 & .54 & $\$ 340$ & Non-Condensing \\
\hline 2 & 1997 & 2020 & .58 & $\$ 370$ & Non-Condensing \\
\hline 3 & 1997 & 2004 & .6 & $\$ 400$ & Non-Condensing \\
\hline 4 & 2005 & 2020 & .6 & $\$ 375$ & Non-Condensing \\
\hline 5 & 1997 & 2020 & .86 & $\$ 2360$ & Non-Condensing \\
\hline 6 & 2005 & 2014 & .86 & $\$ 2000$ & Condensing \\
\hline 7 & 2015 & 2020 & .86 & $\$ 1800$ & Condensing \\
\hline 8 & 2005 & 2014 & .63 & $\$ 450$ & Condensing \\
\hline 9 & 2015 & 2020 & .63 & $\$ 425$ & Non-Condensing \\
\hline 10 & 2015 & 2020 & .7 & $\$ 500$ & Non-Condensing \\
\hline
\end{tabular}

Table D.23. NEMS Results for Energy Star Gas Water Heaters (national market shares for new, single-family homes)

\begin{tabular}{|c|c|c|c|c|}
\hline $\begin{array}{c}\text { Efficiency } \\
\text { Level }\end{array}$ & \multicolumn{2}{|c|}{$\mathbf{2 0 0 5}$} & \multicolumn{2}{c|}{$\mathbf{2 0 1 0}$} \\
\cline { 2 - 5 } & Baseline & $\begin{array}{c}\text { Energy } \\
\text { Star }\end{array}$ & Baseline & $\begin{array}{c}\text { Energy } \\
\text { Star }\end{array}$ \\
\hline 0.60 & 0.307 & 0.387 & 0.315 & 0.384 \\
\hline 0.63 & 0.011 & 0.068 & 0.011 & 0.066 \\
\hline Total & 0.318 & 0.455 & 0.326 & 0.450 \\
\hline
\end{tabular}


Table D.24. NEMS Input Parameters for Room Air Conditioners

\begin{tabular}{|c|c|c|c|c|}
\hline Technology & $\begin{array}{c}\text { Start } \\
\text { Year }\end{array}$ & End Year & SEER & $\begin{array}{c}\text { Installed } \\
\text { Cost }\end{array}$ \\
\hline \multicolumn{5}{|c|}{ AEO 2000 and GPRA Baseline } \\
\hline 1 & 1997 & 2000 & 2.55 & $\$ 450$ \\
\hline 2 & 2001 & 2020 & 2.83 & $\$ 450$ \\
\hline 3 & 1997 & 2004 & 2.93 & $\$ 500$ \\
\hline 4 & 2005 & 2020 & 2.93 & $\$ 490$ \\
\hline 5 & 1997 & 2020 & 3.43 & $\$ 760$ \\
\hline 6 & 2005 & 2020 & 3.43 & $\$ 760$ \\
\hline 7 & 2015 & 2020 & 3.22 & $\$ 600$ \\
\hline \multicolumn{5}{|c|}{ Revised NEMS Inputs for Room Air Conditioners } \\
\hline 1 & 1997 & 2000 & 2.55 & $\$ 450$ \\
\hline 2 & 2001 & 2020 & 2.83 & $\$ 450$ \\
\hline 3 & 1997 & 2004 & 3.11 & $\$ 530$ \\
\hline 4 & 2005 & 2020 & 3.11 & $\$ 520$ \\
\hline 5 & 1997 & 2020 & 3.43 & $\$ 760$ \\
\hline 6 & 2005 & 2020 & 3.52 & $\$ 760$ \\
\hline 7 & 2015 & 2020 & 3.22 & $\$ 600$ \\
\hline
\end{tabular}

Table D.25. NEMS Results for Energy Star Room Air Conditioners (market shares for new, single-family homes)

\begin{tabular}{|c|c|c|c|c|}
\hline & \multicolumn{2}{|c|}{2005} & \multicolumn{2}{c|}{2010} \\
\hline $\begin{array}{c}\text { Census } \\
\text { Division }\end{array}$ & Baseline & $\begin{array}{c}\text { Energy } \\
\text { Star }\end{array}$ & Baseline & $\begin{array}{c}\text { Energy } \\
\text { Star }\end{array}$ \\
\hline 1 & .0083 & .1301 & .0083 & .1299 \\
\hline 2 & .0085 & .1323 & .0085 & .1321 \\
\hline 3 & .0085 & .1319 & .0084 & .1314 \\
\hline 4 & .0084 & .1314 & .0084 & .1312 \\
\hline 5 & .0091 & .1396 & .0091 & .1395 \\
\hline 6 & .0091 & .1402 & .0091 & .1398 \\
\hline 7 & .0101 & .1522 & .0099 & .1501 \\
\hline 8 & .0085 & .1327 & .0085 & .1327 \\
\hline 9 & .0084 & .1314 & .0084 & .1317 \\
\hline
\end{tabular}

Table D.26. Key NEMS Data Inputs for Dishwashers

\begin{tabular}{|c|l|l|l|l|l|}
\hline Technology & $\begin{array}{c}\text { Start } \\
\text { Year }\end{array}$ & $\begin{array}{c}\text { End } \\
\text { Year }\end{array}$ & $\begin{array}{c}\text { Water } \\
\text { Co-efficiency }\end{array}$ & $\begin{array}{c}\text { Energy } \\
\text { Factor }\end{array}$ & $\begin{array}{c}\text { Installed } \\
\text { Cost }\end{array}$ \\
\hline 1 & 1997 & 2020 & .80 & .46 & $\$ 350$ \\
\hline 2 & 1997 & 2004 & .80 & .59 & $\$ 500$ \\
\hline 3 & 2005 & 2020 & .80 & .59 & $\$ 450$ \\
\hline 4 & 1997 & 2004 & .78 & .71 & $\$ 700$ \\
\hline 5 & 2005 & 2014 & .78 & .71 & $\$ 600$ \\
\hline 6 & 2015 & 2020 & .78 & .71 & $\$ 500$ \\
\hline 7 & 2015 & 2020 & .80 & .60 & $\$ 400$ \\
\hline
\end{tabular}


Table D.27. Energy Star Program Dishwashers (estimated market shares for high-efficiency dishwashers)

\begin{tabular}{|c|c|c|c|c|c|c|c|c|}
\hline \multirow{2}{*}{$\begin{array}{c}\text { Census } \\
\text { Division }\end{array}$} & \multicolumn{4}{|c|}{ 2005 } & \multicolumn{3}{c|}{2010} \\
\cline { 2 - 8 } & Baseline & \multicolumn{2}{c|}{ Energy Star } & \multicolumn{2}{c|}{ Baseline } & \multicolumn{2}{c|}{ Energy Star } \\
\hline & $\mathrm{EF}=.59$ & $\mathrm{EF}=.71$ & $\mathrm{EF}=.59$ & $\mathrm{EF}=.71$ & $\mathrm{EF}=.59$ & $\mathrm{EF}=.71$ & $\mathrm{EF}=.59$ & $\mathrm{EF}=.71$ \\
\hline 1 & .0683 & .0012 & .2219 & .0322 & .0682 & .0012 & .2217 & .0321 \\
\hline 2 & .0678 & .0012 & .2207 & .0318 & .0677 & .0012 & .2204 & .0317 \\
\hline 3 & .0659 & .0011 & .2157 & .0305 & .0656 & .0011 & .2151 & .0304 \\
\hline 4 & .0654 & .0011 & .2146 & .0302 & .0654 & .0011 & .2145 & .0304 \\
\hline 5 & .0658 & .0011 & .2156 & .0305 & 30654 & .0011 & .2145 & .0304 \\
\hline 6 & .0655 & .0011 & .2148 & .0303 & .0658 & .0011 & .2156 & .0305 \\
\hline 7 & .0656 & .0011 & .2150 & .0303 & .0653 & .0011 & .2144 & .0302 \\
\hline 8 & .0662 & .0011 & .2166 & .0308 & .0663 & .0012 & .2168 & .0308 \\
\hline 9 & .0661 & .0011 & .2164 & .0307 & .0663 & .0012 & .2169 & .0308 \\
\hline
\end{tabular}

Table D.28. Key NEMS Inputs for Electric Water Heaters -

\begin{tabular}{|c|c|c|c|c|l|}
\hline Technology & $\begin{array}{c}\text { Start } \\
\text { Year }\end{array}$ & End Year & $\begin{array}{c}\text { Energy } \\
\text { Factor }\end{array}$ & $\begin{array}{c}\text { Installed } \\
\text { Cost }\end{array}$ & Type \\
\hline 1 & 1197 & 2003 & 0.86 & $\$ 350$ & Resistance \\
\hline 2 & 1997 & 2003 & 0.88 & $\$ 350$ & Resistance \\
\hline $2 a$ & 2004 & 2020 & 0.89 & $\$ 350$ & Resistance \\
\hline 3 & 1997 & 2020 & 0.95 & $\$ 575$ & Resistance \\
\hline 4 & 1997 & 2020 & 2.60 & $\$ 1,025$ & Heat Pump \\
\hline 5 & 1997 & 2020 & 2.00 & $\$ 2,600$ & Heat Pump \\
\hline 6 & 1997 & 2020 & 0.90 & $\$ 360$ & Resistance \\
\hline 7 & 2005 & 2020 & 0.96 & $\$ 475$ & Resistance \\
\hline 8 & 2004 & 2009 & 2.00 & $\$ 700$ & Heat Pump \\
\hline $8 a$ & 2010 & 2014 & 2.10 & $\$ 650$ & Heat Pump \\
\hline 9 & 2015 & 2020 & 0.90 & $\$ 400$ & Resistance \\
\hline 10 & 2015 & 2020 & 0.96 & $\$ 425$ & Resistance \\
\hline 11 & 2015 & 2020 & 2.20 & $\$ 600$ & Heat Pump* \\
\hline \multicolumn{5}{|l|}{} & \multicolumn{5}{l}{} \\
\hline${ }^{*}$ Emerging Technologies program.
\end{tabular}

Table D.29. NEMS Results for Emerging Technologies Program's Heat Pump Water Heaters (national market shares for new single-family homes)

\begin{tabular}{|c|c|c|}
\hline Year & $\begin{array}{c}\text { Market Share: } \\
\text { AEO 2000 Discount } \\
\text { Rate }\end{array}$ & $\begin{array}{c}\text { Market Share: } \\
\text { Adjusted Discount } \\
\text { Rate (Energy Star) }\end{array}$ \\
\hline 2004 & 0.024 & 0.040 \\
\hline 2005 & 0.012 & 0.031 \\
\hline 2006 & 0.012 & 0.050 \\
\hline 2007 & 0.012 & 0.077 \\
\hline 2008 & 0.012 & 0.116 \\
\hline 2010 & 0.028 & 0.239 \\
\hline 2015 & 0.047 & 0.241 \\
\hline 2020 & 0.048 & 0.243 \\
\hline
\end{tabular}


Table D.30. Key NEMS Inputs for Gas Water Heaters

\begin{tabular}{|c|c|c|c|c|l|}
\hline Technology & Start Yr & End Yr & Energy Factor & Installed Cost & \multicolumn{1}{|c|}{ Type } \\
\hline 1 & 1997 & 2003 & 0.54 & $\$ 340$ & Non-condensing \\
\hline 2 & 1997 & 2003 & 0.58 & $\$ 370$ & Non-condensing \\
\hline 3 & 1997 & 2004 & 0.60 & $\$ 400$ & Non-condensing \\
\hline 4 & 2005 & 2020 & 0.60 & $\$ 375$ & Non-condensing \\
\hline 5 & 1997 & 2020 & 0.86 & $\$ 2,360$ & Condensing \\
\hline 6 & 2005 & 2014 & 0.86 & $\$ 2,000$ & Condensing \\
\hline 7 & 2015 & 2020 & 0.86 & $\$ 1,800$ & Condensing \\
\hline 8 & 2005 & 2014 & 0.63 & $\$ 450$ & Non-condensing \\
\hline 9 & 2015 & 2020 & 0.63 & $\$ 425$ & Non-condensing \\
\hline 10 & 2015 & 2020 & 0.70 & $\$ 500$ & Non-condensing \\
\hline 11 & 2005 & 2009 & 0.80 & $\$ 550$ & Condensing--ET Pgm \\
\hline 12 & 2010 & 2020 & 0.8 & $\$ 525$ & Condensing--ET Pgm \\
\hline
\end{tabular}

Table D.31. NEMS Results for Energy Star Gas Water Heaters (national market shares for new single-family homes)

\begin{tabular}{|c|c|c|}
\hline Year & $\begin{array}{c}\text { Market Share: } \\
\text { AEO 2000 } \\
\text { Discount Rate }\end{array}$ & $\begin{array}{c}\text { Market Share: } \\
\text { Adjusted } \\
\text { Discount Rate }\end{array}$ \\
\hline 2005 & 0.003 & 0.009 \\
\hline 2006 & 0.003 & 0.015 \\
\hline 2007 & 0.003 & 0.024 \\
\hline 2008 & 0.003 & 0.038 \\
\hline 2010 & 0.011 & 0.129 \\
\hline 2015 & 0.009 & 0.100 \\
\hline 2020 & 0.010 & 0.106 \\
\hline
\end{tabular}


Table D.32. Building Simulation Parameters

\begin{tabular}{|c|c|c|c|}
\hline Building Type & $\begin{array}{c}\text { Building Size } \\
\left(\mathrm{ft}^{2}\right)\end{array}$ & $\begin{array}{c}\text { Vintage (Year } \\
\text { Built) }\end{array}$ & Location \\
\hline Assembly & \multirow{10}{*}{$\begin{array}{l}4000 \\
7500 \\
17500 \\
37500 \\
75000 \\
125000\end{array}$} & \multirow{13}{*}{$\begin{array}{l}1940 \\
1953 \\
1967 \\
1976 \\
1983 \\
2000\end{array}$} & \multirow{13}{*}{$\begin{array}{l}\text { Denver, Colorado } \\
\text { Detroit, Michigan } \\
\text { Fresno, California } \\
\text { Knoxville, Tennessee } \\
\text { Los Angeles, California } \\
\text { Minneapolis, Minnesota } \\
\text { Phoenix, Arizona } \\
\text { Providence, Rhode Island } \\
\text { Seattle, Washington } \\
\text { Shreveport, Louisiana } \\
\text { Tampa, Florida }\end{array}$} \\
\hline Education & & & \\
\hline Food Sales & & & \\
\hline Food Service & & & \\
\hline Healthcare & & & \\
\hline Lodging & & & \\
\hline Mercantile and Service & & & \\
\hline Office & & & \\
\hline Warehouse & & & \\
\hline $\begin{array}{l}\text { Other Commercial } \\
\text { Buildings }\end{array}$ & & & \\
\hline Single Family & \multirow[b]{2}{*}{$\begin{array}{l}600 \\
800 \\
1300 \\
1800 \\
2200 \\
3000^{*} \\
\end{array}$} & & \\
\hline Mobile Home & & & \\
\hline Multifamily & $\begin{array}{l}14309 \\
19079 \\
31003 \\
42927 \\
52466 \\
71545\end{array}$ & & \\
\hline
\end{tabular}

\section{D.33. Weights Given to Each City for Each Census Region (\%)}

\begin{tabular}{|l|c|c|c|c|c|c|c|c|c|}
\hline \multicolumn{1}{|c|}{ City } & $\begin{array}{c}\text { New } \\
\text { England }\end{array}$ & $\begin{array}{c}\text { Mid } \\
\text { Atlantic }\end{array}$ & $\begin{array}{c}\text { East } \\
\text { North } \\
\text { Central }\end{array}$ & $\begin{array}{c}\text { West } \\
\text { North } \\
\text { Central }\end{array}$ & $\begin{array}{c}\text { South } \\
\text { Atlantic }\end{array}$ & $\begin{array}{c}\text { East } \\
\text { South } \\
\text { Central }\end{array}$ & $\begin{array}{c}\text { West } \\
\text { South } \\
\text { Central }\end{array}$ & Mountain & Pacific \\
\hline Denver & 0.0 & 0.0 & 0.0 & 0.0 & 0.0 & 0.0 & 0.0 & 64.0 & 2.2 \\
\hline Detroit & 0.0 & 0.0 & 99.3 & 60.0 & 0.0 & 0.0 & 0.0 & 0.0 & 0.0 \\
\hline Fresno & 0.0 & 0.0 & 0.0 & 0.0 & 0.0 & 0.0 & 0.0 & 0.0 & 13.3 \\
\hline Knoxville & 0.0 & 0.0 & 0.0 & 0.0 & 50.7 & 67.4 & 13.4 & 0.0 & 0.0 \\
\hline Los Angeles & 0.0 & 0.0 & 0.0 & 0.0 & 0.0 & 0.0 & 0.0 & 0.0 & 71.2 \\
\hline Minneapolis & 0.0 & 0.0 & 0.7 & 40.0 & 0.0 & 0.0 & 0.0 & 0.0 & 0.0 \\
\hline Phoenix & 0.0 & 0.0 & 0.0 & 0.0 & 0.0 & 0.0 & 0.0 & 36.0 & 0.0 \\
\hline Providence & 100.0 & 100.0 & 0.0 & 0.0 & 0.0 & 0.0 & 0.0 & 0.0 & 0.0 \\
\hline Seattle & 0.0 & 0.0 & 0.0 & 0.0 & 0.0 & 0.0 & 0.0 & 0.0 & 13.2 \\
\hline Shreveport & 0.0 & 0.0 & 0.0 & 0.0 & 17.7 & 32.6 & 80.6 & 0.0 & 0.0 \\
\hline Tampa & 0.0 & 0.0 & 0.0 & 0.0 & 31.6 & 0.0 & 6.0 & 0.0 & 0.0 \\
\hline
\end{tabular}


Table D.34. Fraction of Floor Space In Each Size Category for Each Commercial Building Type

\begin{tabular}{|l|c|c|c|c|c|c|}
\hline \multirow{2}{*}{\multicolumn{1}{c|}{ Building Type }} & \multicolumn{7}{|c|}{ Floor Space } \\
\cline { 2 - 7 } & $\mathbf{4 , 0 0 0}$ & $\mathbf{7 , 5 0 0}$ & $\mathbf{1 7 , 5 0 0}$ & $\mathbf{3 7 , 5 0 0}$ & $\mathbf{7 5 , 0 0 0}$ & $\mathbf{1 2 5 , 0 0 0}$ \\
\hline Assembly & 7.9 & 19.9 & 23.8 & 12.3 & 12.6 & 23.5 \\
\hline Education & 3.2 & 5.2 & 13.5 & 23.6 & 22.6 & 31.8 \\
\hline Food Sales & 36.4 & 6.4 & 31.8 & 19.1 & 5.1 & 1.3 \\
\hline Food Service & 40.7 & 28.8 & 24.4 & 5.2 & 0.6 & 0.3 \\
\hline Healthcare & 6.5 & 6.5 & 10.4 & 7.5 & 5.5 & 63.6 \\
\hline Lodging & 4.1 & 7.4 & 20.7 & 14.2 & 16.9 & 36.7 \\
\hline Mercantile and Service & 14.5 & 17.3 & 23.1 & 9.3 & 10.0 & 25.7 \\
\hline Large Office & 0.0 & 0.0 & 0.0 & 0.0 & 27.5 & 72.5 \\
\hline Small Office & 21.7 & 18.9 & 32.7 & 26.7 & 0.0 & 0.0 \\
\hline Other Buildings & 10.8 & 12.8 & 19.7 & 13.0 & 13.5 & 30.1 \\
\hline Warehouse & 9.5 & 11.7 & 18.0 & 13.7 & 13.5 & 33.5 \\
\hline
\end{tabular}

Table D.35. Fraction of Floor Space in Each Size Category for Each Residential Type (\%)

\begin{tabular}{|l|c|c|c|c|c|c|}
\hline \multirow{2}{*}{ Building Type } & \multicolumn{7}{|c|}{ Floor Space } \\
\cline { 2 - 7 } & $\mathbf{6 0 0}$ & $\mathbf{8 0 0}$ & $\mathbf{1 3 0 0}$ & $\mathbf{1 8 0 0}$ & $\mathbf{2 2 0 0}$ & $\mathbf{3 0 0 0}$ \\
\hline Single Family & 2.8 & 14.0 & 37.0 & 21.2 & 11.3 & 13.7 \\
\hline Mobile Home & 15.7 & 43.8 & 31.6 & 7.2 & 2.2 & 0.7 \\
\hline & \multicolumn{7}{|c|}{ Floor Space } \\
\hline & $\mathbf{1 4 , 3 0 9}$ & $\mathbf{1 9 , 0 7 9}$ & $\mathbf{3 1 , 0 0 3}$ & $\mathbf{4 2 , 9 2 7}$ & $\mathbf{5 2 , 4 6 6}$ & $\mathbf{7 1 , 5 4 5}$ \\
\hline Multifamily & 25.4 & 49.3 & 17.9 & 2.4 & 0.7 & 0.2 \\
\hline
\end{tabular}

Table D.36. Market Penetration Estimates

\begin{tabular}{|l|l|l|l|c|c|c|c|c|}
\hline \multirow{2}{*}{ Program } & Sector & Vintage & 1st Year & \multicolumn{2}{|c|}{ Penetration in Yr (\%) } & External & Internal \\
\cline { 5 - 8 } & & & of Pen. & $\mathbf{2 0 0 5}$ & $\mathbf{2 0 2 0}$ & $\mathbf{2 0 3 0}$ & Param. & Param. \\
\hline Superwindows & Residential & Existing & 2003 & 1.5 & 33.0 & 43.2 & 0.008 & 0.2435 \\
\hline Superwindows & Residential & New & 2003 & 3.0 & 65.0 & 85.0 & 0.008 & 0.2435 \\
\hline $\begin{array}{l}\text { Electrochromic } \\
\text { Windows }\end{array}$ & Commercial & Existing & 2001 & 1.8 & 17.2 & 43.2 & 0.0022 & 0.111 \\
\hline $\begin{array}{l}\text { Electrochromic } \\
\text { Windows }\end{array}$ & Commercial & New & 2001 & 2.0 & 20.0 & 50.0 & 0.0022 & 0.111 \\
\hline R30-30 roof & Commercial & Existing & 2010 & 0.0 & 15.3 & 30.2 & 0.01 & 0.3 \\
\hline R30-30 roof & Commercial & New & 2010 & 0.0 & 21.5 & 42.2 & 0.01 & 0.3 \\
\hline Quick Fill Wall & Commercial & Existing & 2004 & 0.0 & 0.0 & 0.0 & 0.01 & 0.3 \\
\hline Quick Fill Wall & Commercial & New & 2004 & 0.2 & 6.3 & 7.2 & 0.01 & 0.3 \\
\hline Quick Fill Wall & Residential & Existing & 2004 & 0.0 & 0.0 & 0.0 & 0.01 & 0.3 \\
\hline Quick Fill Wall & Residential & New & 2004 & 0.3 & 10.0 & 11.5 & 0.01 & 0.3 \\
\hline
\end{tabular}

